University of South Florida

DIGITAL COMMONS

@ UNIVERSITY OF SOUTH FLORIDA
Digital Commons @ University of South Florida

\title{
$6-2015$
}

\section{Development of Public Transit II Course}

CUTR

Follow this and additional works at: https://digitalcommons.usf.edu/cutr_nctr

\section{Scholar Commons Citation}

CUTR, "Development of Public Transit II Course" (2015). Research Reports. 189.

https://digitalcommons.usf.edu/cutr_nctr/189

This Technical Report is brought to you for free and open access by the National Center for Transit Research (NCTR) Archive (2000-2020) at Digital Commons @ University of South Florida. It has been accepted for inclusion in Research Reports by an authorized administrator of Digital Commons @ University of South Florida. For more information, please contact digitalcommons@usf.edu. 


\title{
Development of Public Transit II Course
}

\author{
Prepared By \\ Jeremy Mattson and Ranjit Godavarthy \\ North Dakota State University
}

\author{
Project Number \\ 79060-02-A
}

\begin{abstract}
Prepared For
National Center for Transit Research

Center for Urban Transportation Research
\end{abstract}

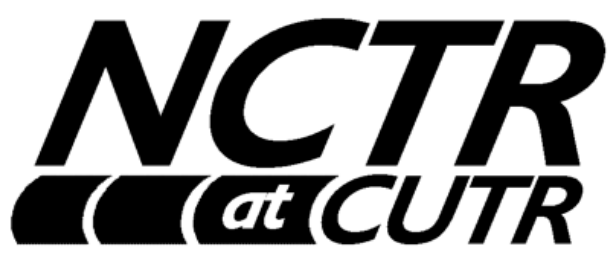

June 2015

\section{CUTR.USF.EDU}




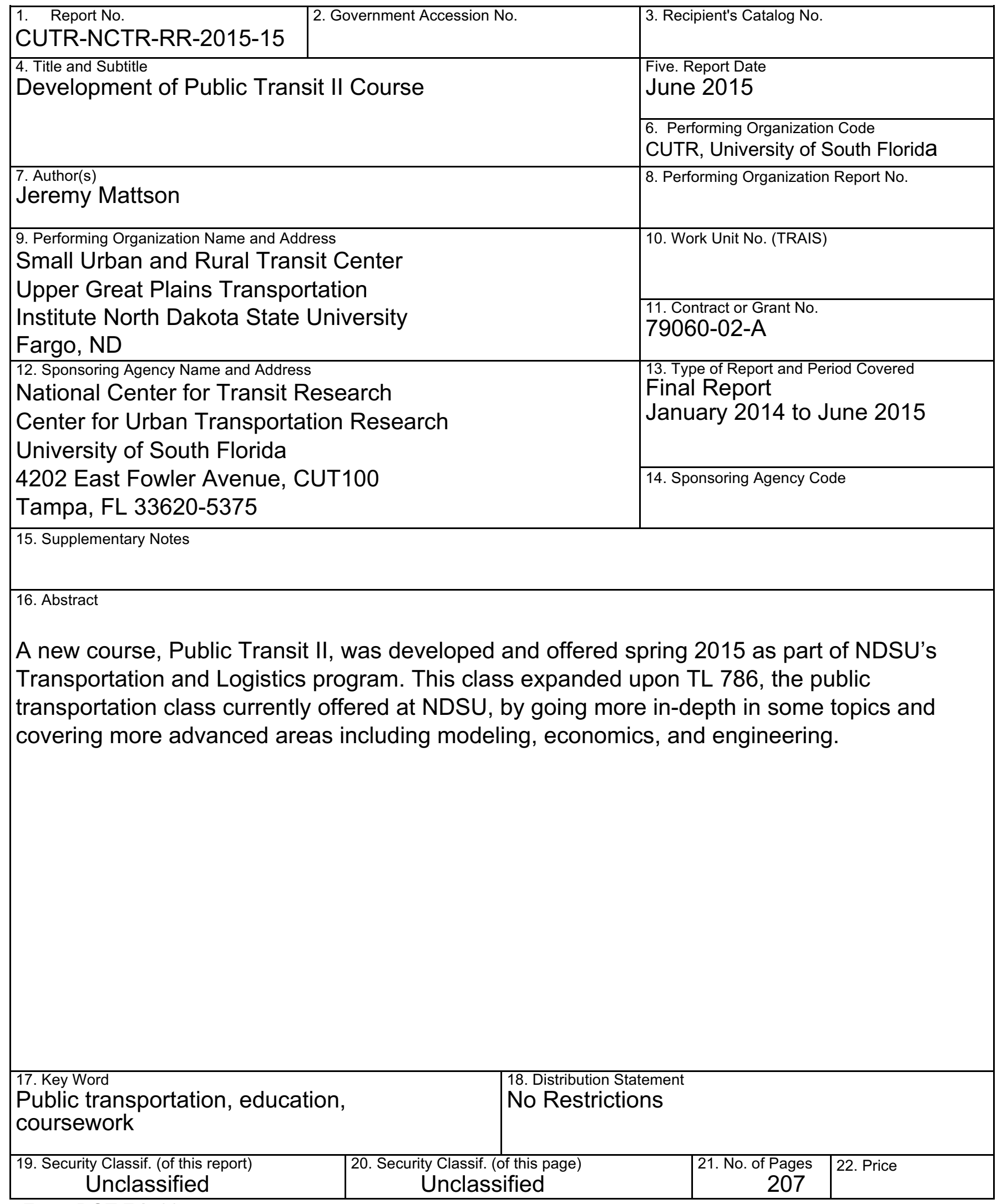

Form DOT F 1700.7 (8-72) Reproduction of completed page authorized 


\section{Disclaimer}

Final report must include this disclaimer: The contents of this report reflect the views of the authors, who are responsible for the facts and the accuracy of the information presented herein. This document is disseminated under the sponsorship of the U.S. Department of Transportation's University Transportation Centers Program, in the interest of information exchange. The U.S. Government assumes no liability for the contents or use thereof.

\section{Acknowledgment}

This work was supported by the National Center for Transit Research, a program of the Center for Urban Transportation Research at the University of South Florida and funded by the U.S. Department of Transportation. 
SYLLABUS 


\section{TL 787 Public Transportation II: 3 Credits}

\section{Instructors and contact information}

Dr. Ranjit Godavarthy, 410B Quentin Burdick Building, Phone: 701.231.6436

Email: ranjit.godavarthy@ndsu.edu

Office hours: By appointment

Mr. Jeremy Mattson, 410C Quentin Burdick Building, Phone: 701.231.5496

Email: jeremy.w.mattson@ndsu.edu

Office hours: By appointment

\section{Course description}

This course focuses on concepts and modeling procedures used when planning and operating public transportation systems. Topics covered include transit demand analysis, quality of service concepts and estimation, bus and rail capacity, and service planning.

\section{Course objectives}

The objective of this course is to provide the student with an understanding of models used when planning and operating public transportation systems. Students will learn methods for estimating transit demand, quality of service, and capacity, as well as tools for designing service. Students will learn the modeling techniques and how to apply them to real-world scenarios.

\section{Prerequisite}

TL 786 - Special allowances can be made for those with a background in public transportation who have not taken TL 786.

\section{Evaluation procedures and criteria}

Grades will be based on the following:

Homework assignments

Online discussions / class participation

Exams

Final paper / project
25 percent

10 percent

30 percent

35 percent

The following scale will be used to assign letter grades. 


\begin{tabular}{|c|c|}
\hline Numerical Score & Letter Grade \\
\hline $90-100$ & A \\
\hline $80-89$ & B \\
\hline $70-79$ & C \\
\hline $60-69$ & D \\
\hline Below 60 & F \\
\hline
\end{tabular}

Homework: Students will be given weekly homework assignments, which will consist of problems or short essay questions. Assignments are due at the start of class on the date indicated, and the solutions will be discussed in class.

Exams: Exams are expected to be a combination of problems and essay questions.

Final paper/project: Students will be assigned a class project to be due by the end of the semester. At the end of the semester, students will make a presentation reporting their project. More details will be given to students early in the semester.

\section{Attendance}

According to NDSU Policy 333, attendance in classes is expected. Class attendance is not a direct component of the course grade, but participation in class and online discussion boards accounts for $10 \%$ of the grade. Students unable to participate in class are expected to make up for it by participating in the online discussion boards. If you cannot attend a class, it is a courtesy to inform your instructor in advance if possible. Late assignments will have an automatic $10 \%$ deducted. 


\section{Course schedule / calendar of events}

\begin{tabular}{|l|l|l|}
\hline \multicolumn{1}{|c|}{ Week } & Topic & References \\
\hline Jan 11-15 & Introduction, Mode and Service Concepts & TCQSM Chapters 2 \\
\hline Jan 18-22 & Operations Concepts & TCQSM Chapter 3 \\
\hline Jan 25-29 & Transit Operations and Service Scheduling & Vuchic Chapter 1 \\
\hline Feb 1-5 & Demand Analysis & $\begin{array}{l}\text { Balcombe Chapters 3 \& 5, Ceder } \\
\text { Chapter 11; TCRP 161 }\end{array}$ \\
\hline Feb 8-12 & Quality of Service: Concepts and Methods & TCQSM Chapters 4, 5 \\
\hline Feb 15-19 & Bus Transit Capacity & TCQSM Chapter 6 \\
\hline Feb 22-26 & Bus Transit Capacity & TCQSM Chapter 6 \\
\hline Feb 29-Mar 4 & Demand Response Transit Capacity & TCQSM Chapter 7 \\
\hline Mar 7-9 & Mid-term exam (No lecture) & \\
\hline Mar 14-18 & No Class - NDSU Spring Break & TCQSM Chapter 8 \\
\hline Mar 21-25 & Rail Transit Capacity & AREMA Material \\
\hline Mar 28-Apr 1 & Railroad Engineering & Vuchic Chapter 4 \\
\hline Apr 4-8 & Service Planning & Walker - Human Transit \\
\hline Apr 11-15 & Transit Service Planning Issues & $\begin{array}{l}\text { Innovative Funding Sources for } \\
\text { Transit, Kockelman et al., TCRP } \\
\text { 63 }\end{array}$ \\
\hline Apr 18-22 & Transit Financing, Transit Surveys & \\
\hline Apr 25-29 & Presentations & No class \\
\hline May 2-6 & Final exam, class project due & \\
\hline May 9-13 & & \\
\hline
\end{tabular}




\section{Required student resources}

Students must have access to a computer and the internet. The instructor's lecture notes will be made available. Additional references will be identified on the web.

No textbook is required for this course - handouts will be made available. The following resources are available online or will be provided to students.

- Transit Capacity and Quality of Service Manual (TCQSM), Third Edition. TCRP Report 165. Transit Cooperative Research Program, Washington, DC: Transportation Research Board, 2013. http://www.trb.org/main/blurbs/169437.aspx

- Vuchic, V. Urban Transit: Operations, Planning and Economics. John Wiley and Sons, New Jersey. (2005)

- Balcombe et al. The Demand for Public Transit: A Practical Guide. TRL 2004.

- Innovative Funding Sources for Transit, September 2012.

- TCRP Report 161. Methods for Forecasting Demand and Quantifying Need for Rural Passenger Transportation: Final Workbook. Transit Cooperative Research Program, Washington, DC: Transportation Research Board, 2013. http://www.trb.org/Main/Blurbs/168758.aspx

- TCRP Synthesis 63. On-Board and Intercept Transit Survey Techniques. Transit Cooperative Research Program, Washington, DC: Transportation Research Board, 2002. http://www.trb.org/Main/Blurbs/156542.aspx

- TCRP Synthesis 69. Web-Based Survey Techniques: A Synthesis of Transit Practice, Transit Cooperative Research Program, Washington, DC: Transportation Research Board, 2006. www.tcrponline.org/PDFDocuments/tsyn69.pdf

\section{Optional Reading}

- Ceder, A. Public Transit Planning and Operation: Theory, Modeling and Practice (Second Edition) Butterworth-Heinemann. (2014)

- Litman, Todd. 2004. "Transit Price Elasticities and Cross-Elasticities," Journal of Public Transportation 7(2):37-58.

- Litman, Todd. (2009). Transportation Cost and Benefit Analysis: Techniques, Estimates and Implications, Second Edition. Victoria Transport Policy Institute. http://www.vtpi.org/tca/

- Walker, Jarrett. Human Transit: How Clearer Thinking about Public Transit Can Enrich Our Communities and Our Lives. Washington, DC: Island Press, 2012 


\section{Disability statement}

Any students with disabilities or other special needs, who need special accommodations in this course are invited to share these concerns or requests with the instructor and contact the Disability Services Office as soon as possible.

\section{Academic honesty statement}

The academic community is operated on the basis of honesty, integrity, and fair play. NDSU Policy 335: Code of Academic Responsibility and Conduct applies to cases in which cheating, plagiarism, or other academic misconduct have occurred in an instructional context. Students found guilty of academic misconduct are subject to penalties, up to and possibly including suspension and/or expulsion. Student academic misconduct records are maintained by the Office of Registration and Records. Informational resources about academic honesty for students and instructional staff members can be found at $\underline{w w w . n d s u . e d u / a c a d e m i c h o n e s t y}$ 


\section{LECTURE NOTES}



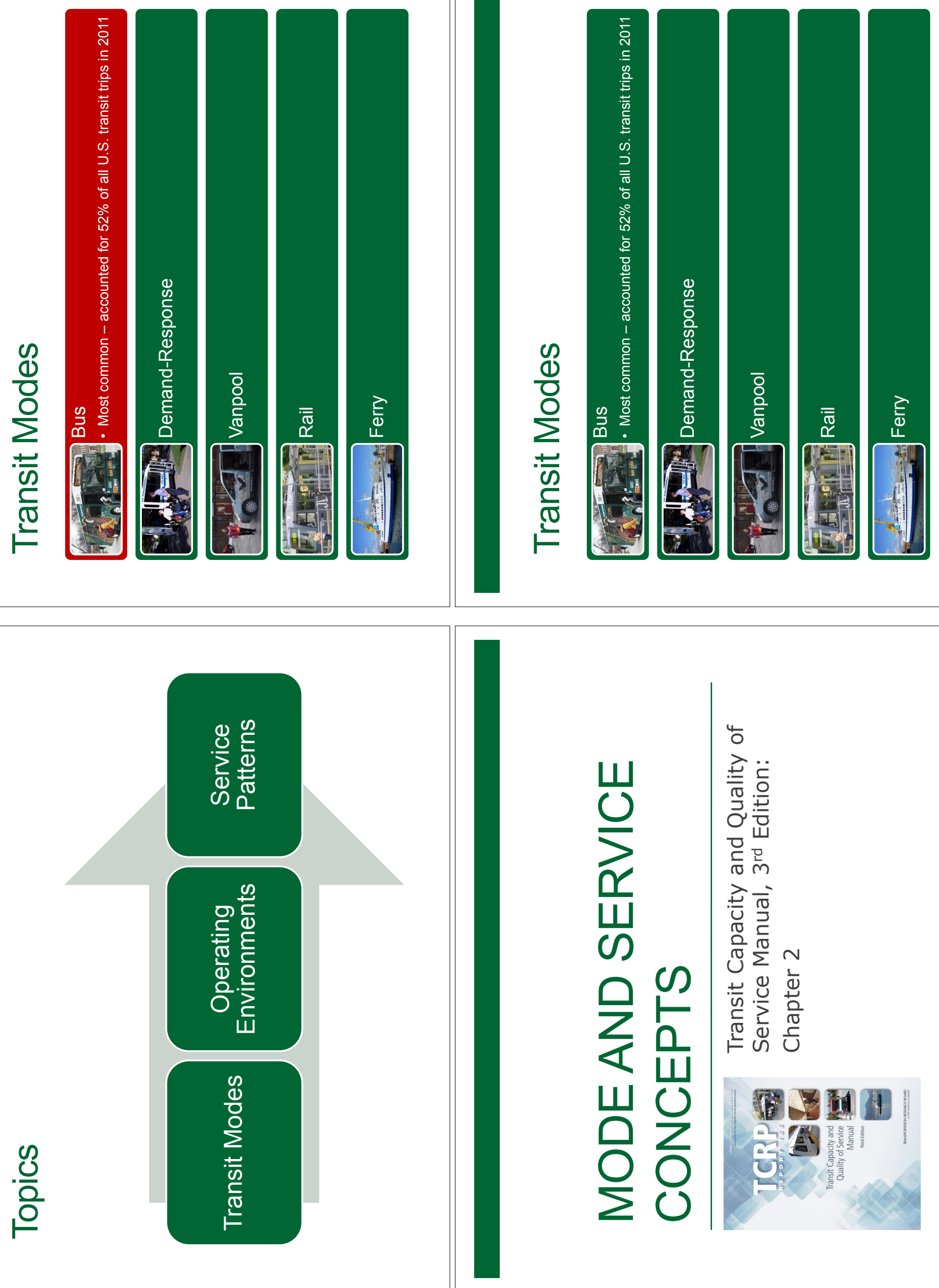

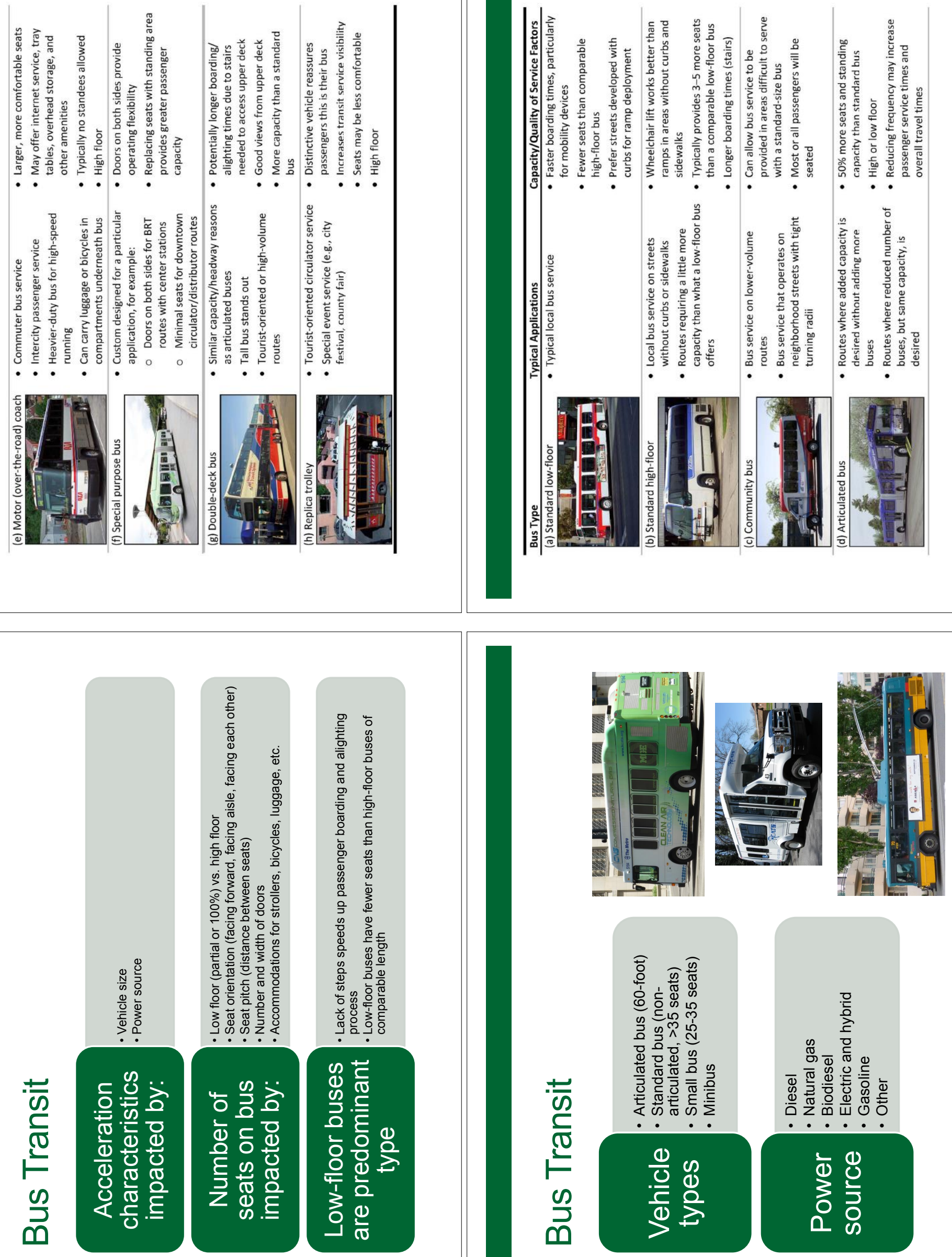

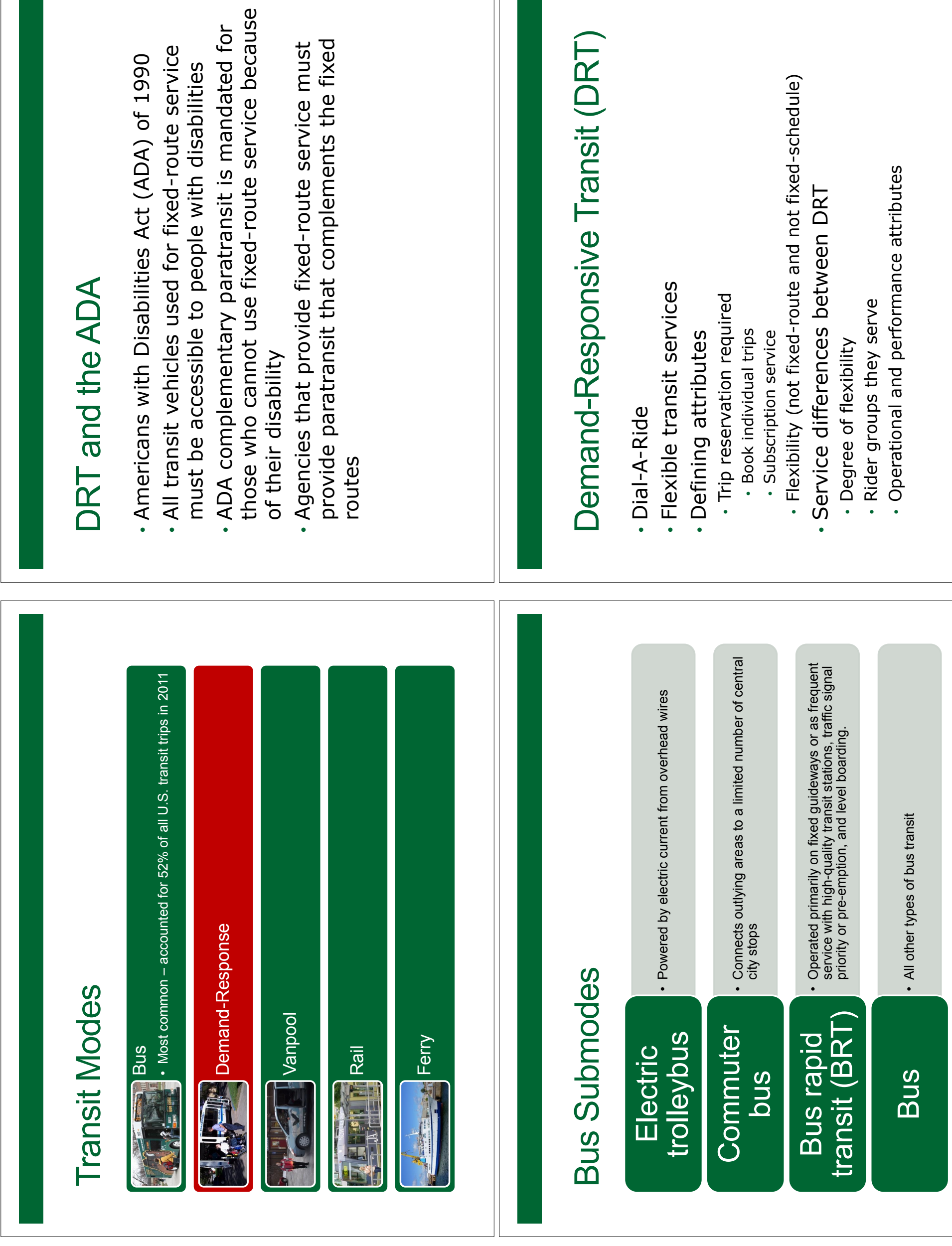
등

는

บํํำ

শ

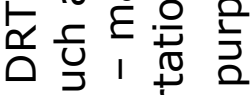

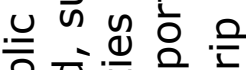

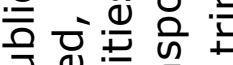

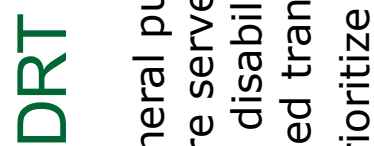

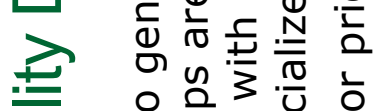

언 을 ญ

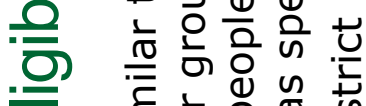

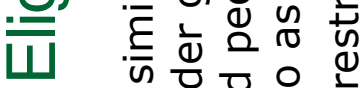

(1)

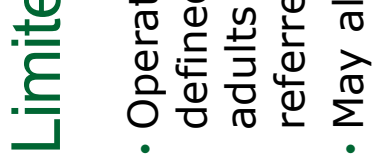

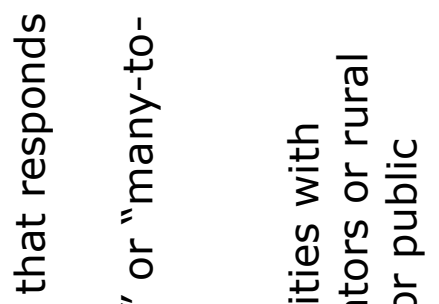

ชิ

ये⿱一兀)

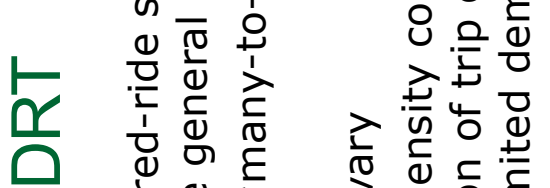

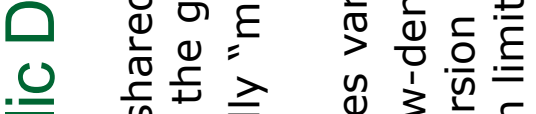

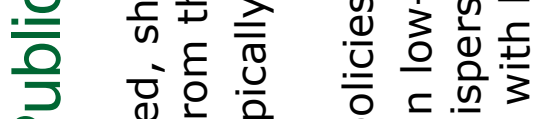

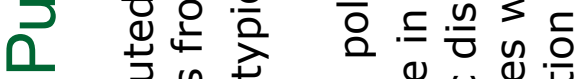

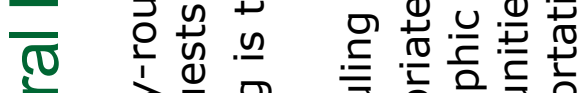

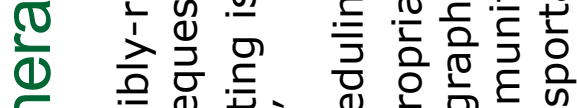

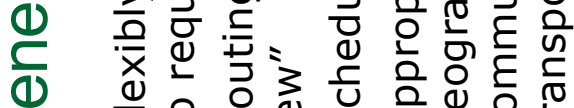

(1) एक 언
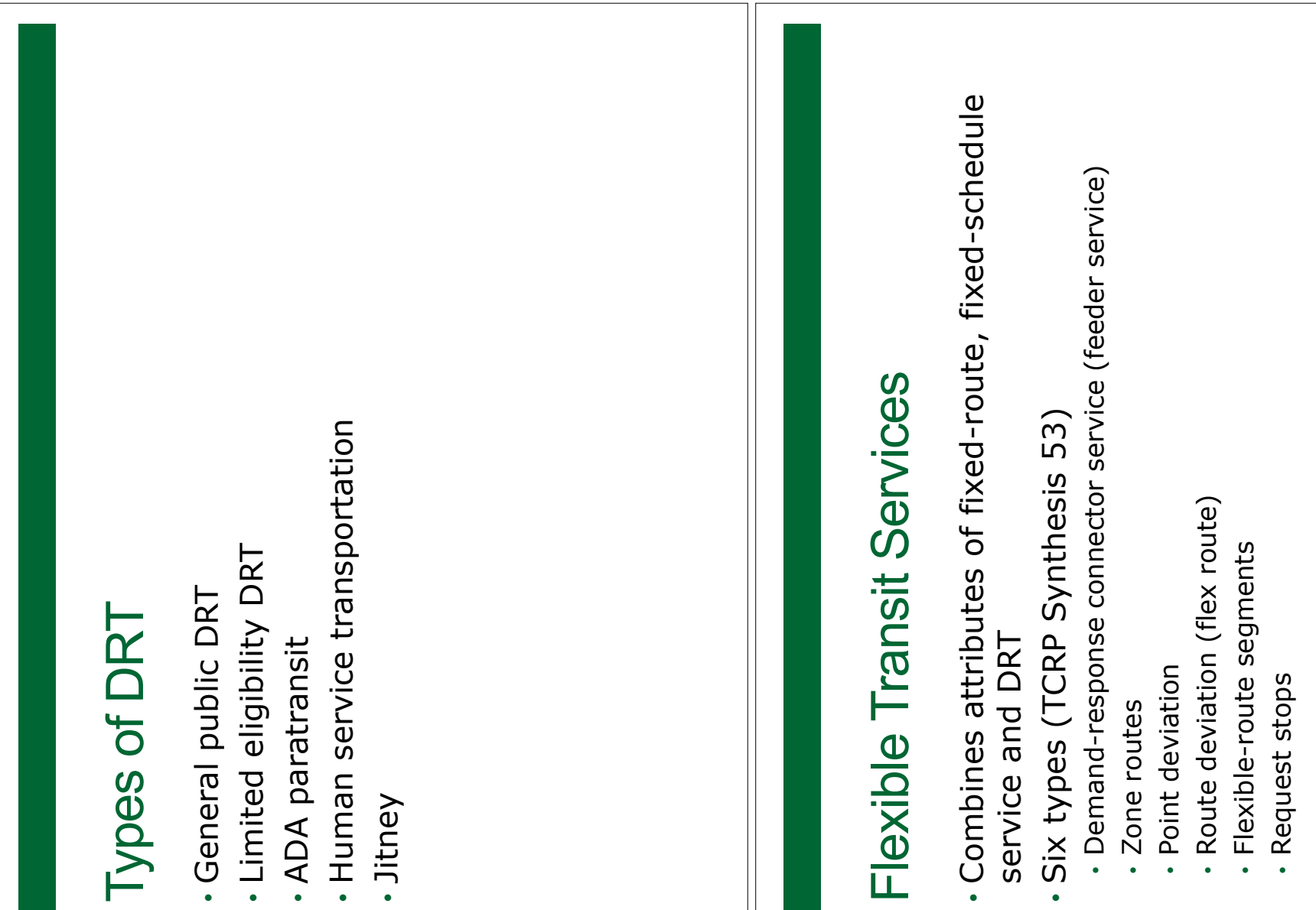


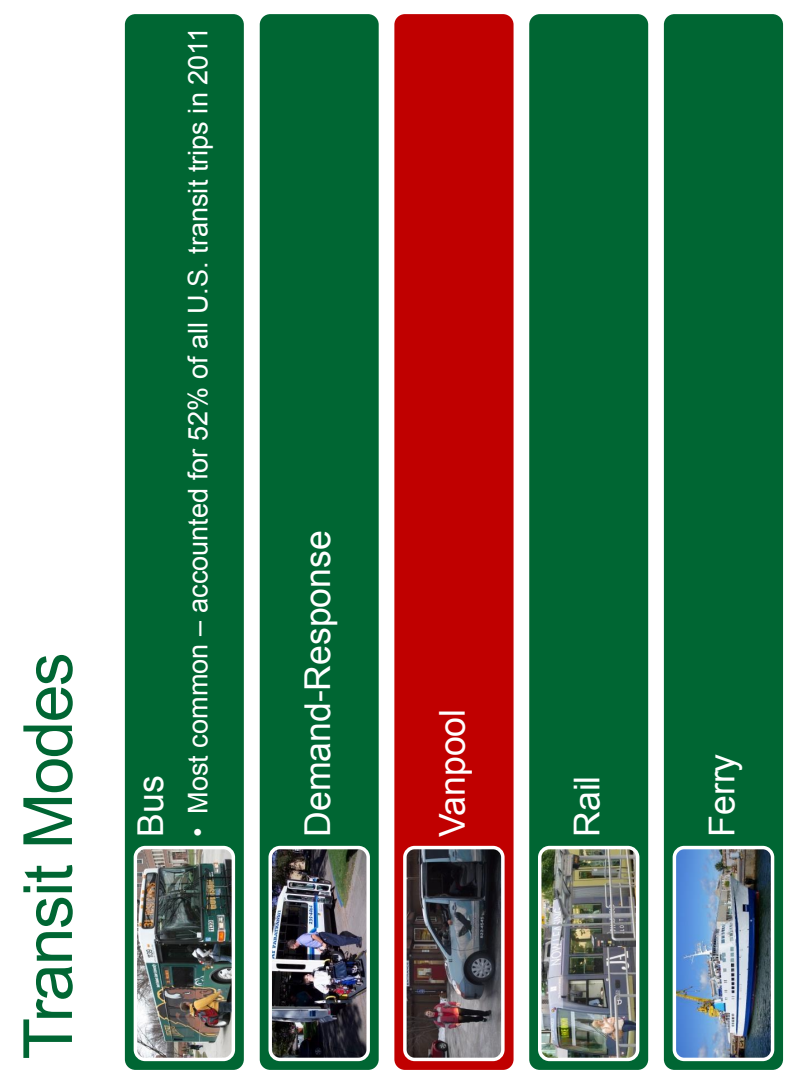

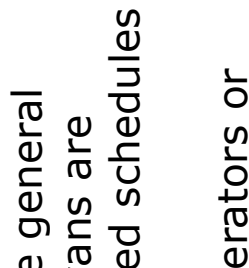

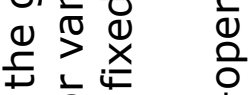

엄늘

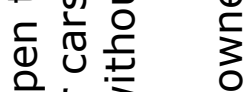

㐫㐫

宁过 艺 U

태오 으 ำ

ษ

ㄷํ워ำ

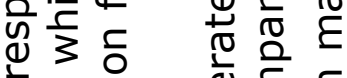

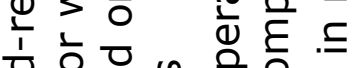

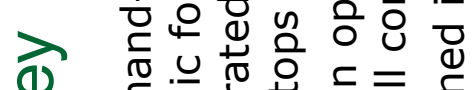

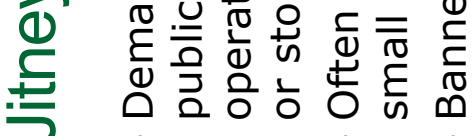

흔

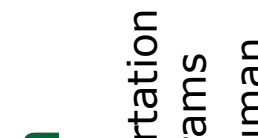

ᄃ 능 는

을 늘 을

प्र

응 믄 궁

ผ गั

तั

느 บै

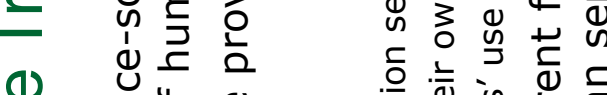

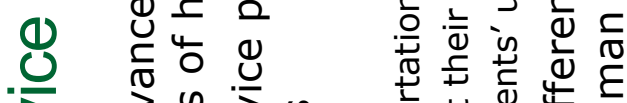

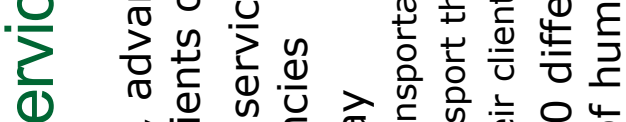

(1) ब

()

ฮั

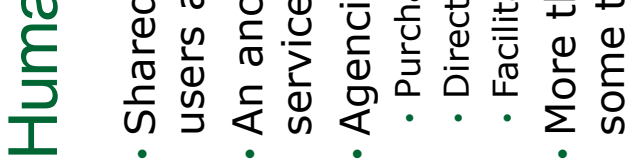

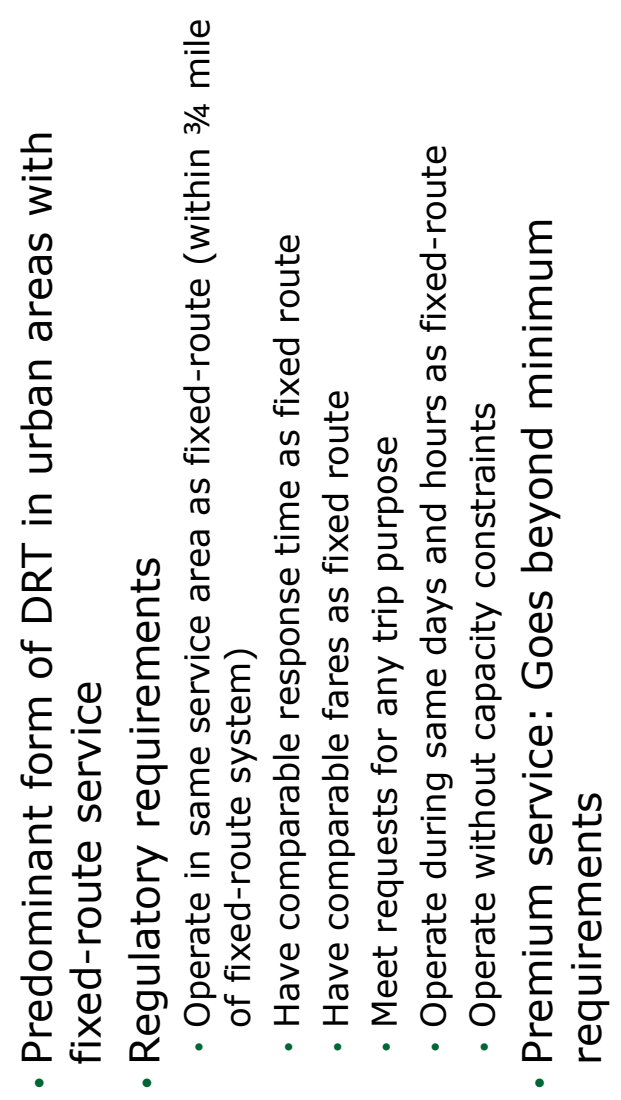




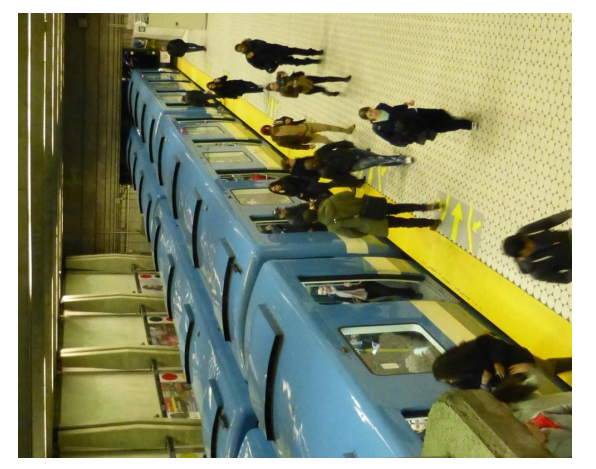

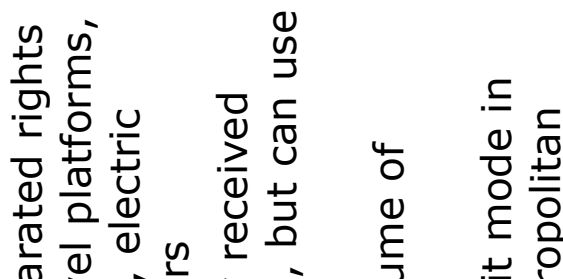

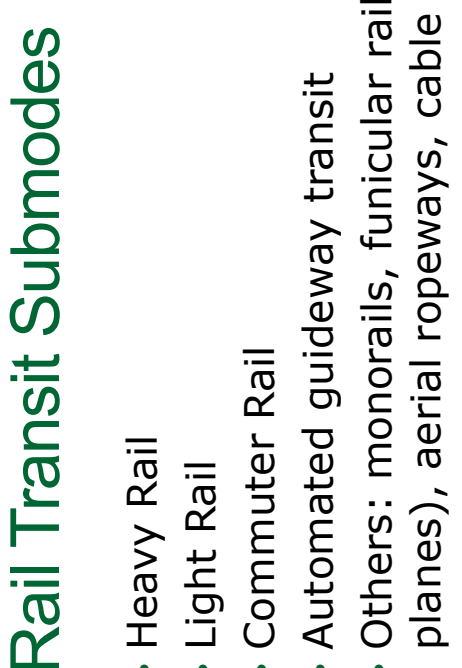

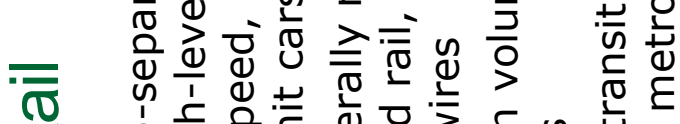

(2)

$\alpha \quad \frac{0}{0}$ 向

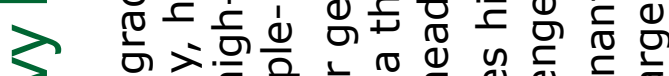

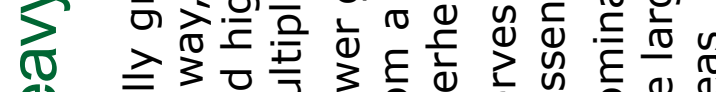

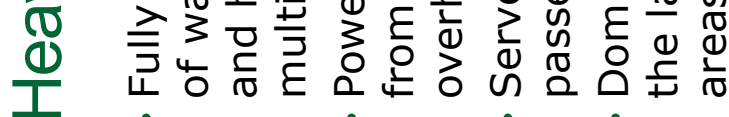

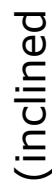

n n

त $\frac{1}{3}$

$\bar{\sigma}$

$\frac{8}{\frac{1}{0}}$

$\frac{\pi}{\bar{N}} \frac{1}{\frac{\pi}{0}}$

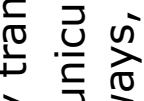

ते

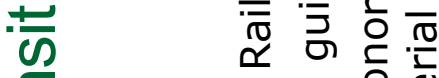

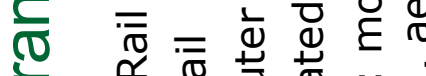

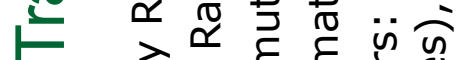

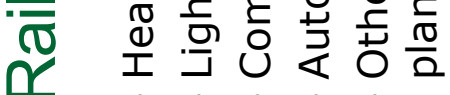

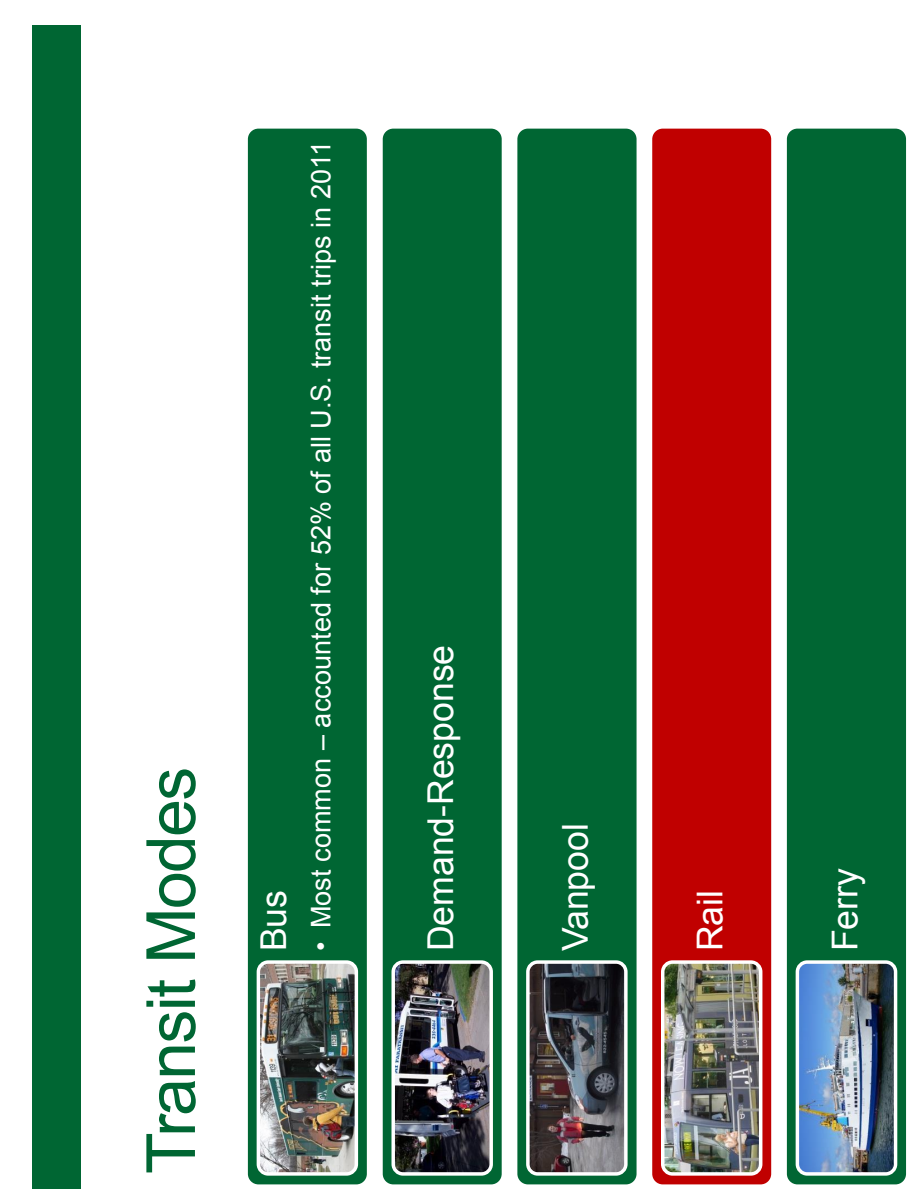

ते

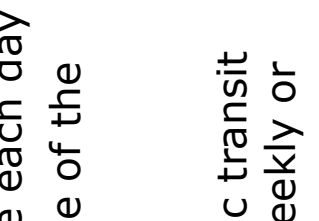

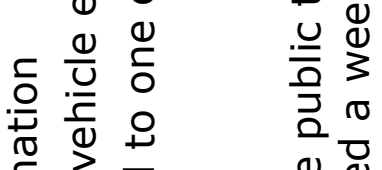

竞

ป ป

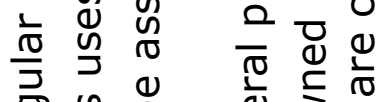

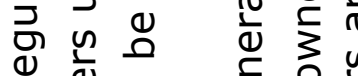

1) 㐫

ס 은 正

언

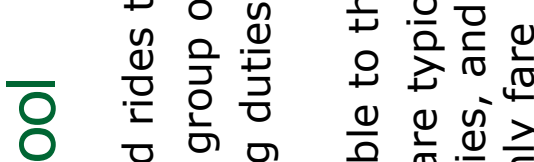

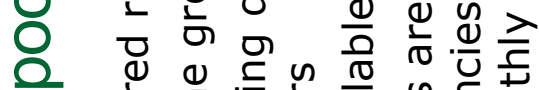

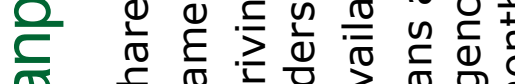

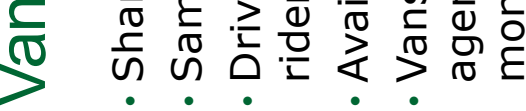



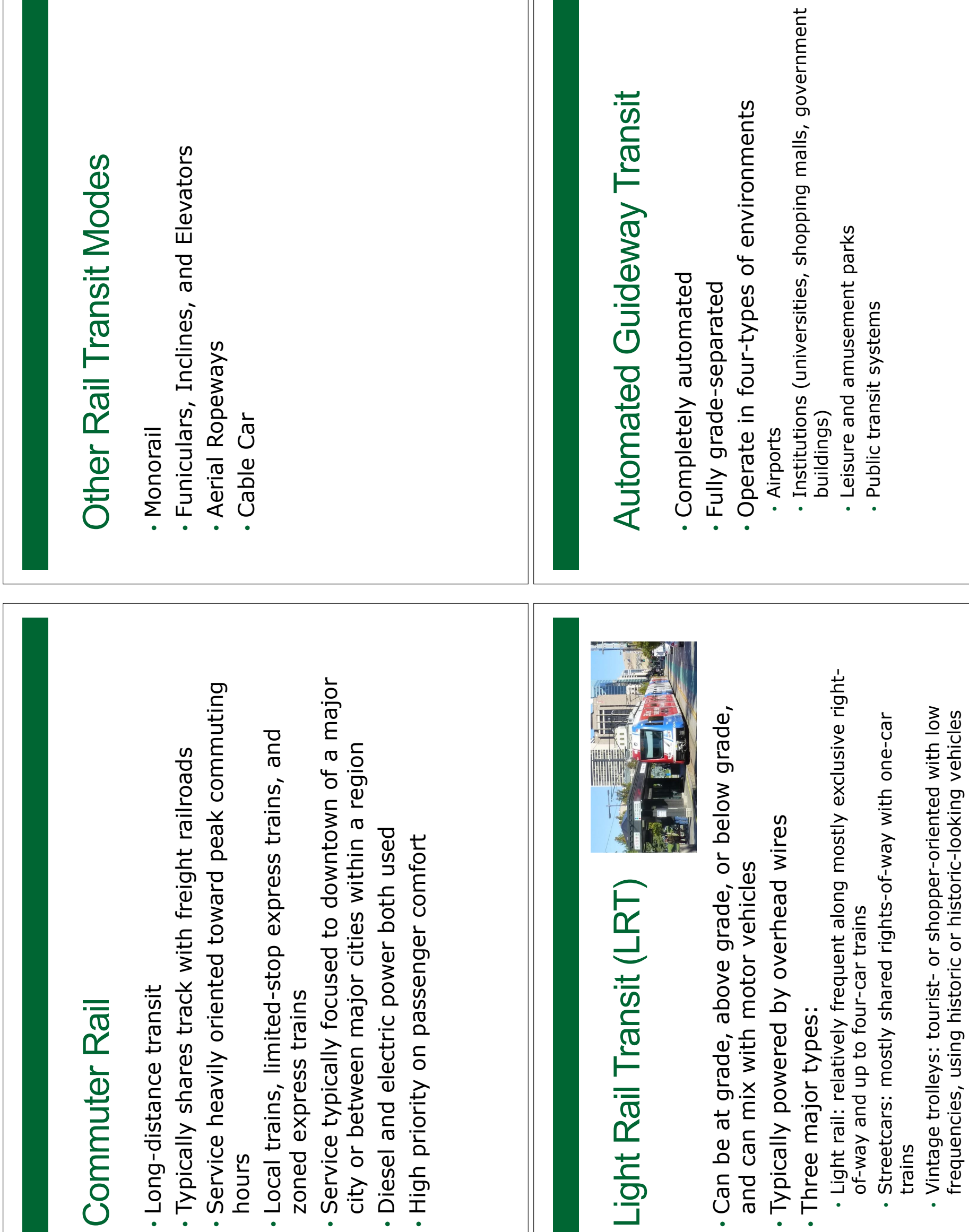

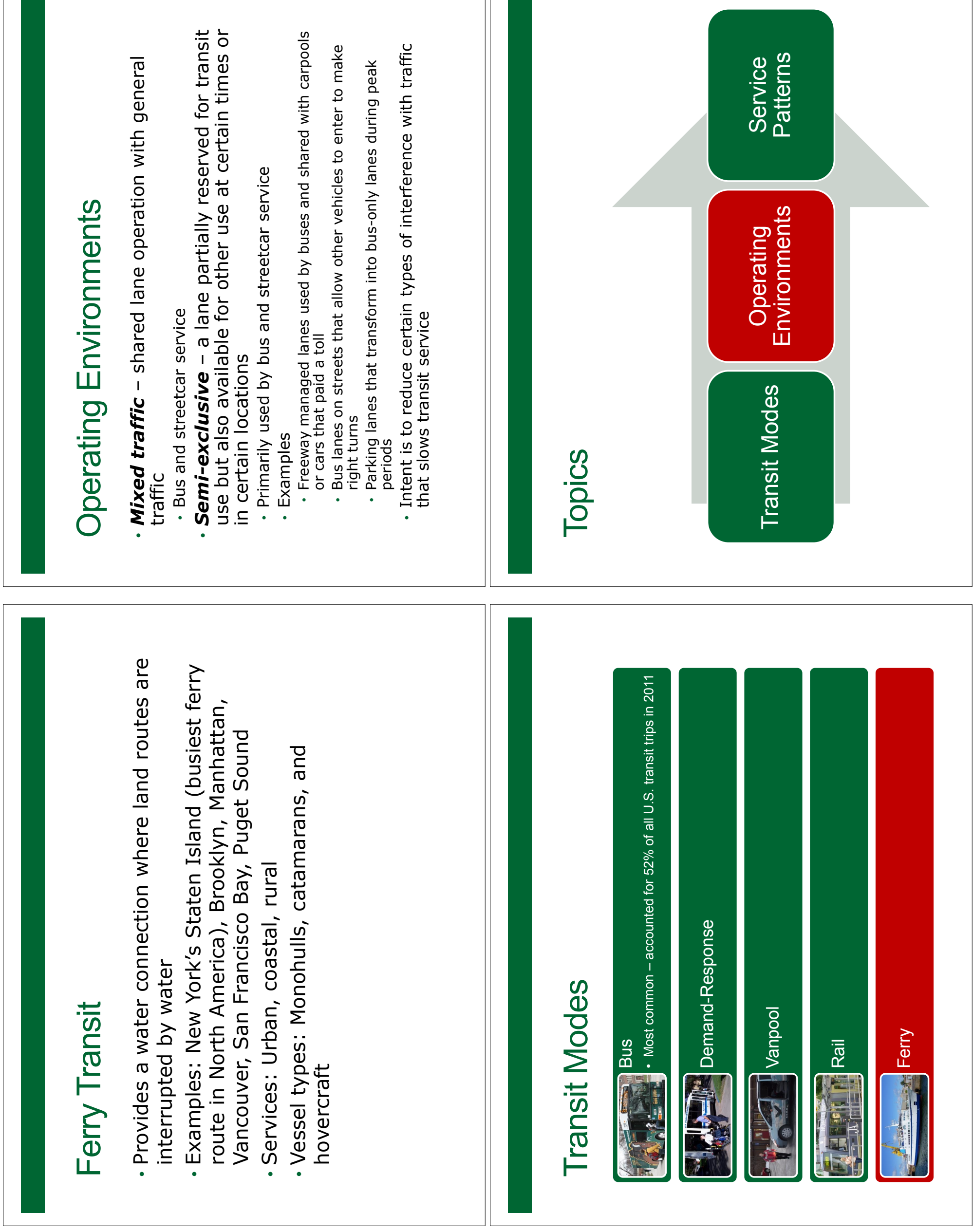

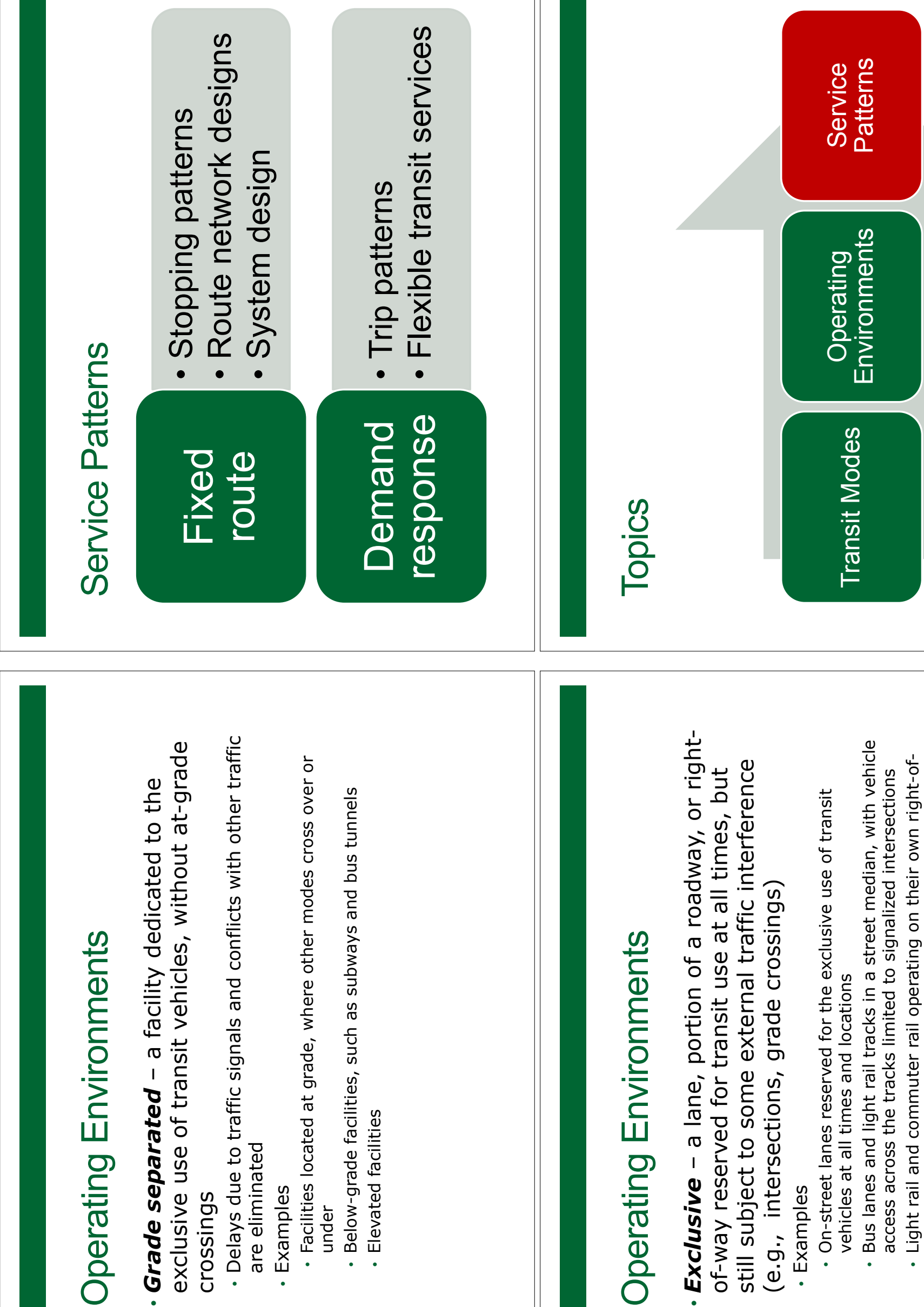

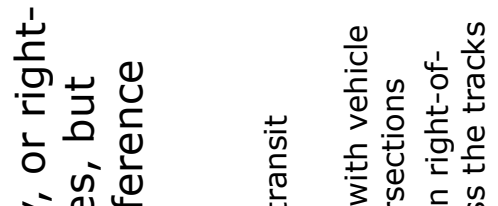

文导坒

方声点

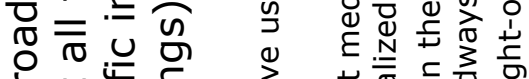

( )

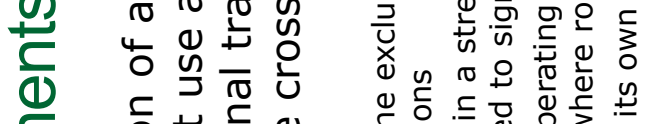

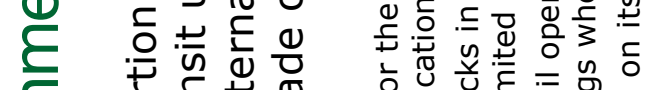

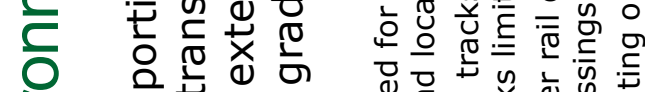

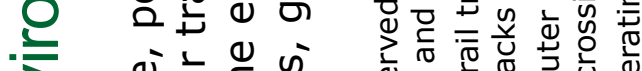

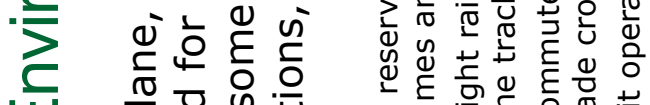

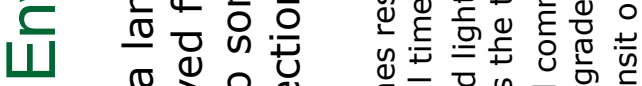

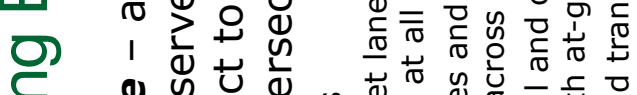

=

(1) ท

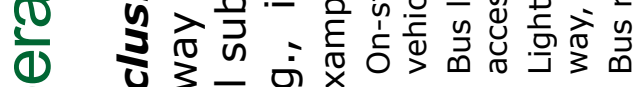

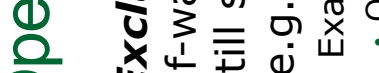

0 u 

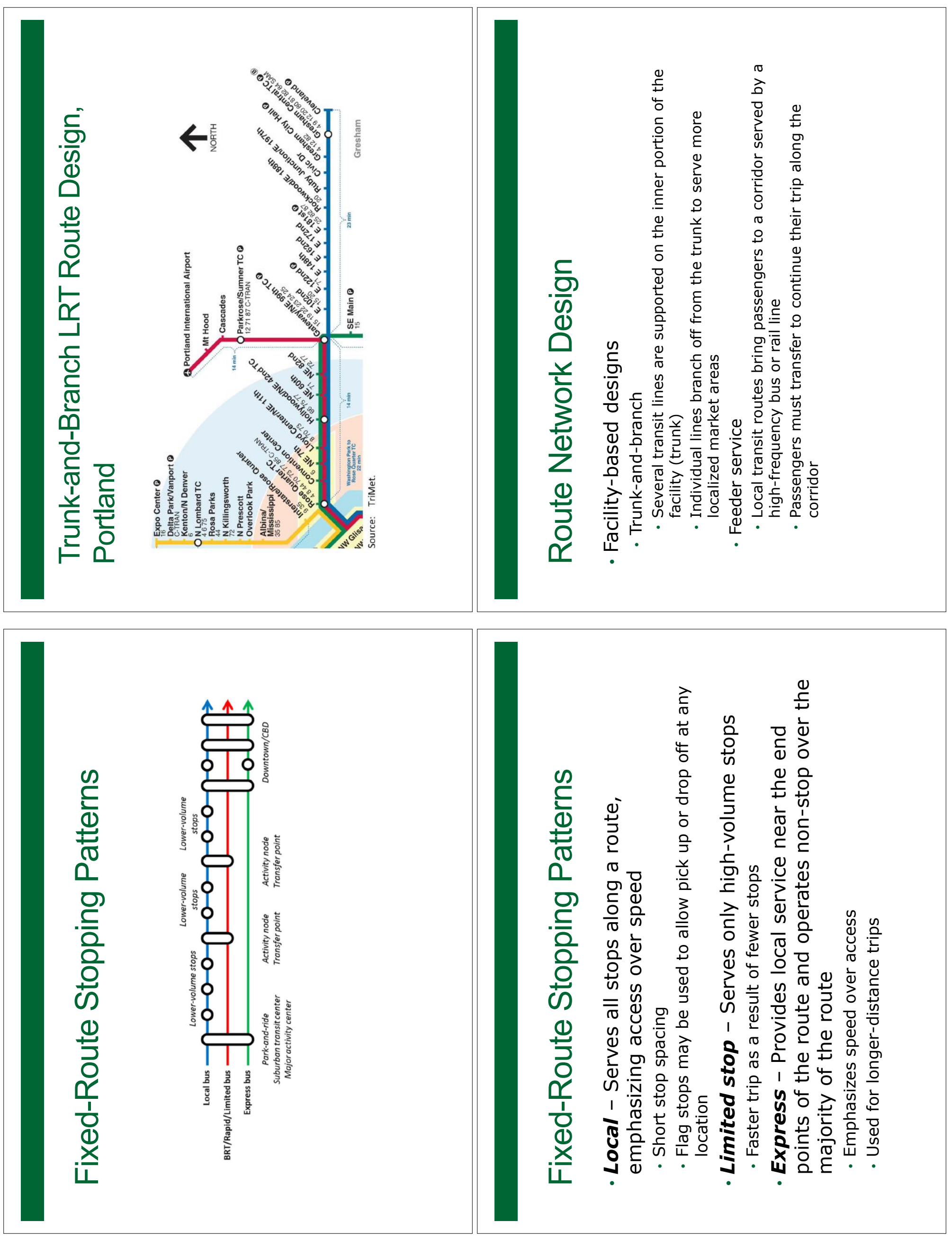

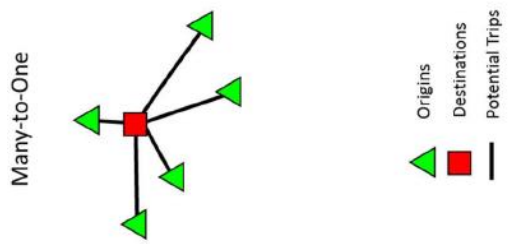

0 2

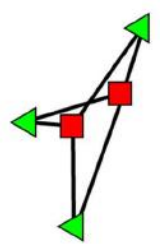

ชิ

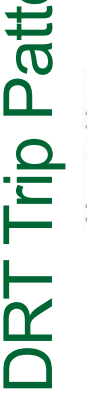

$\stackrel{\infty}{\frac{0}{6}}$

(1)

$\cdot \frac{0}{2}$

(1)

(1)

() $\quad$ 吾

С $\quad$ वै

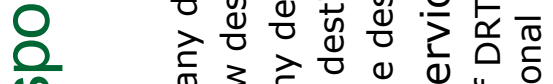

ज) है

(1) 1 ㅇำ

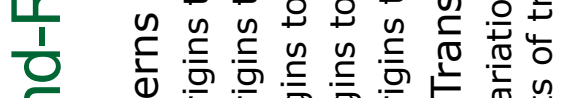

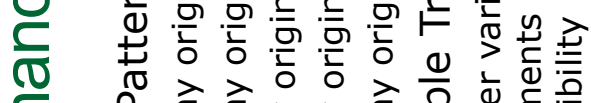

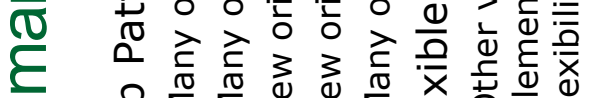

过 은 $\sum_{0}^{\pi} \sum_{0}^{\pi}$

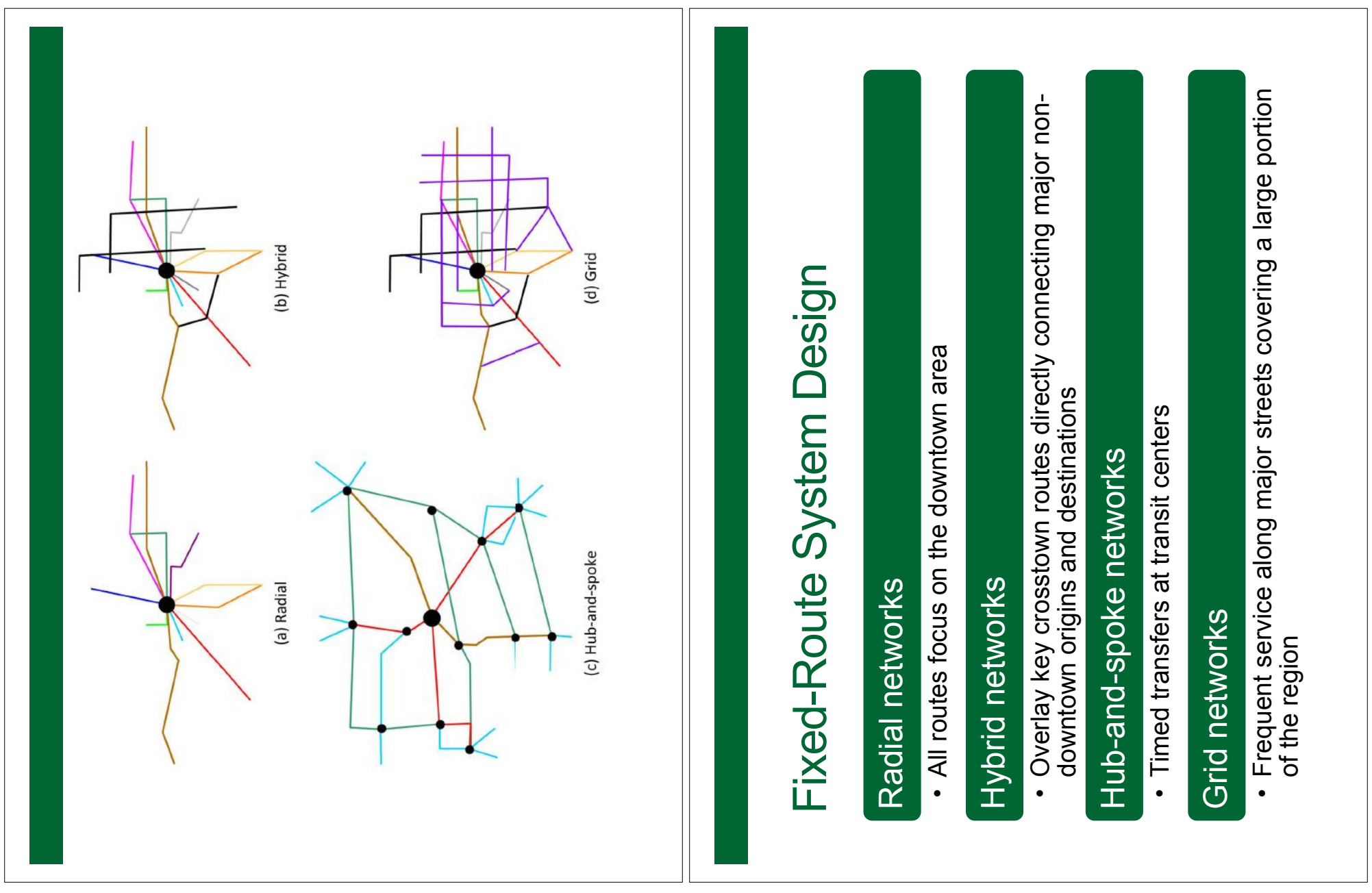




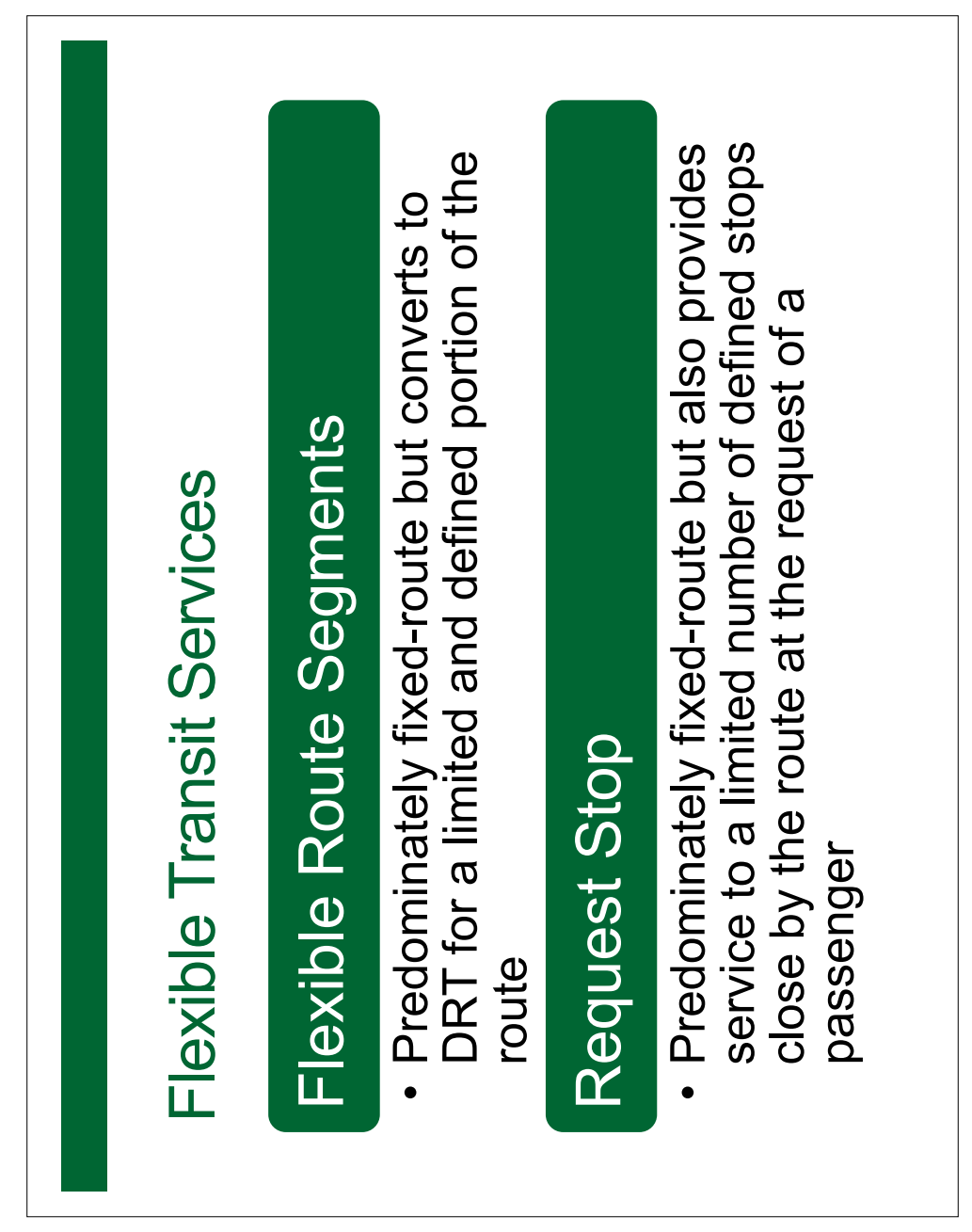

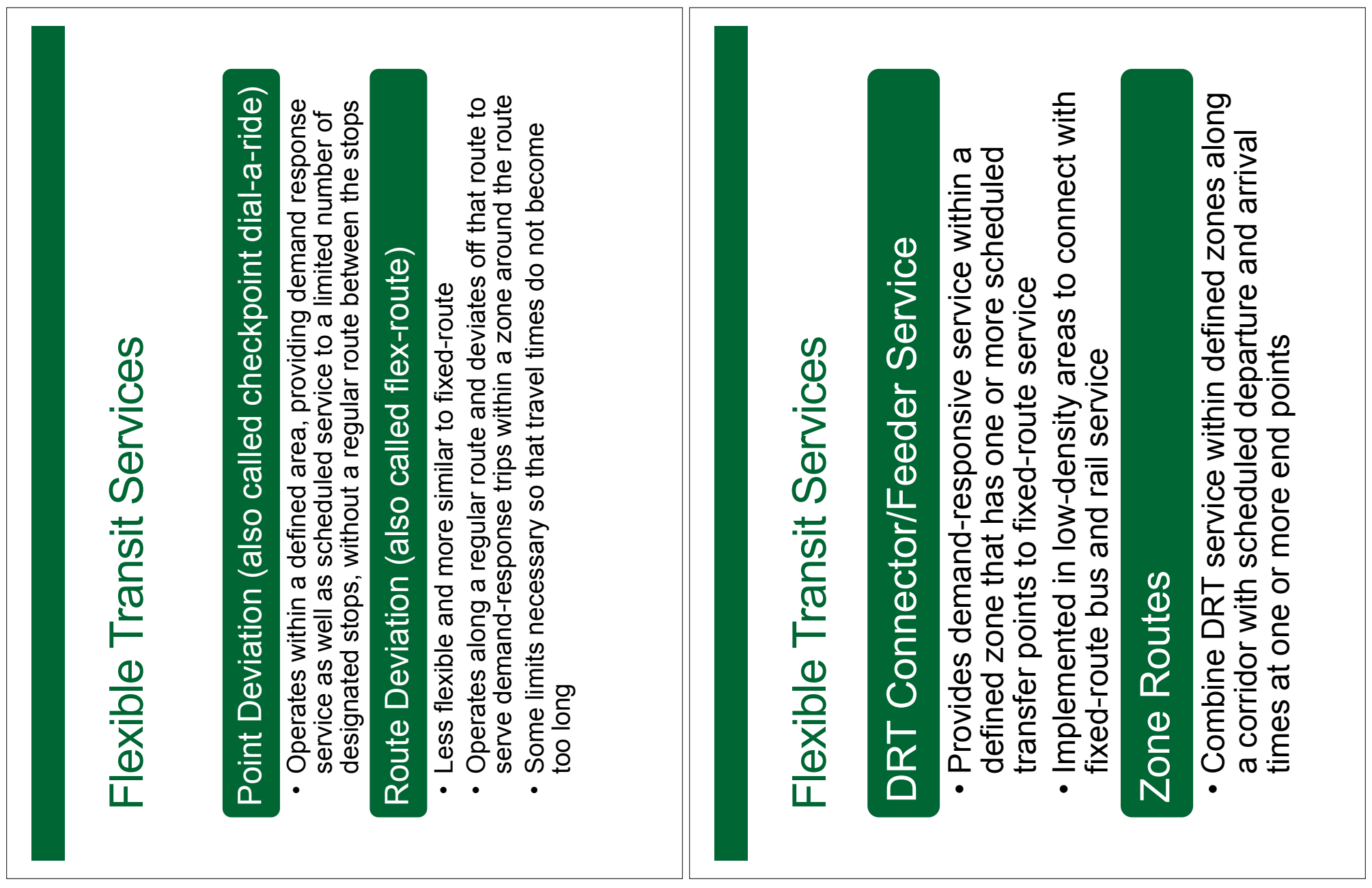



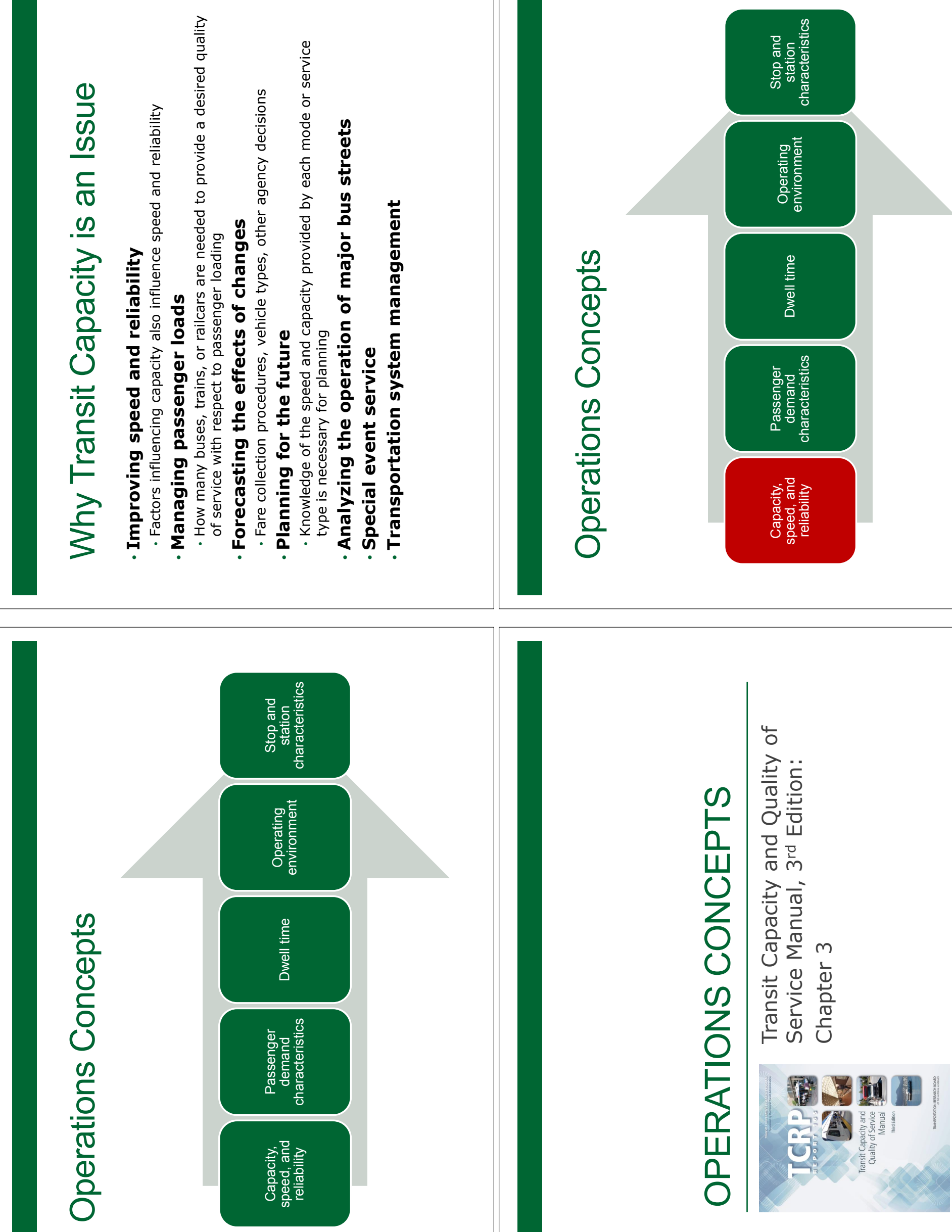

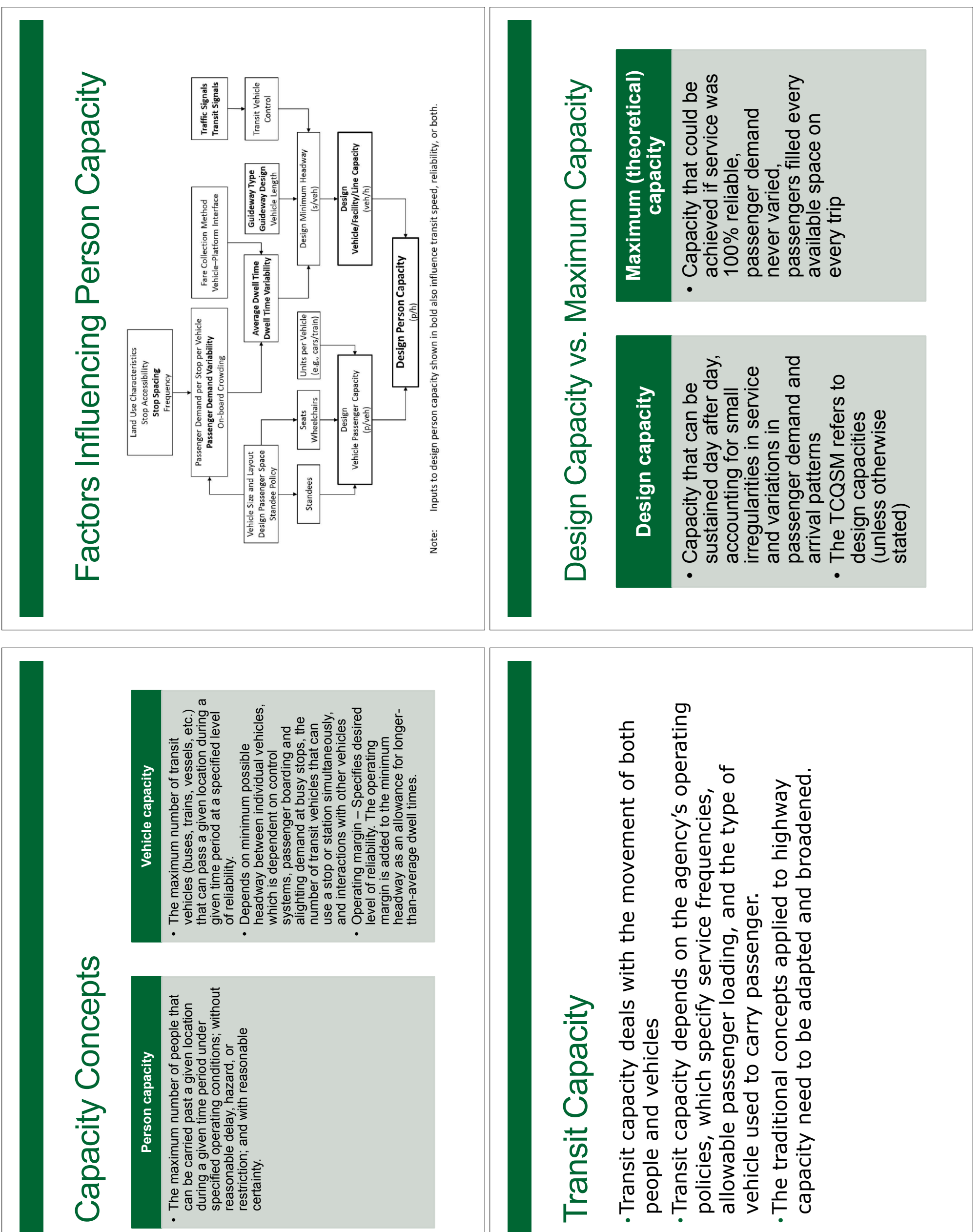

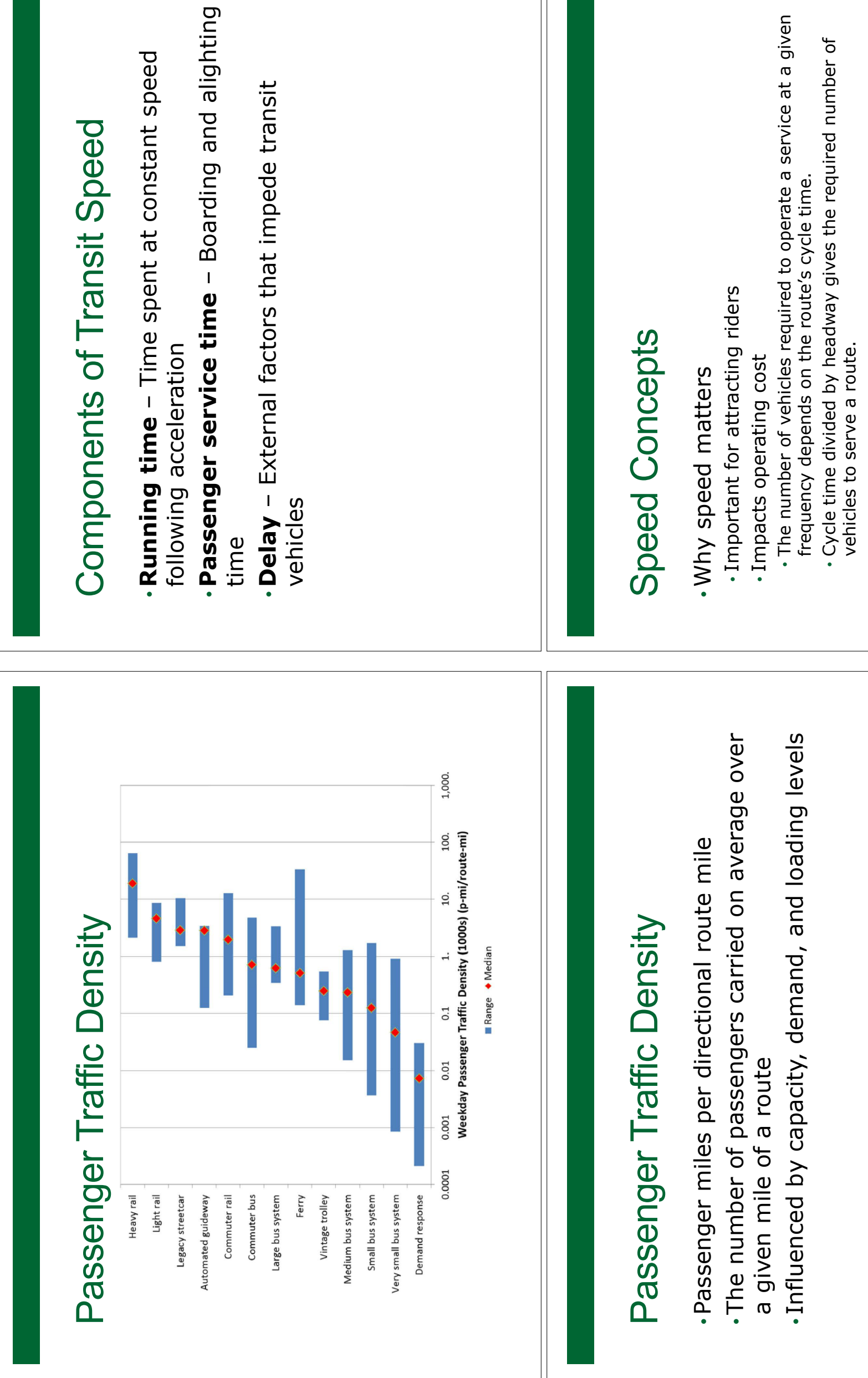

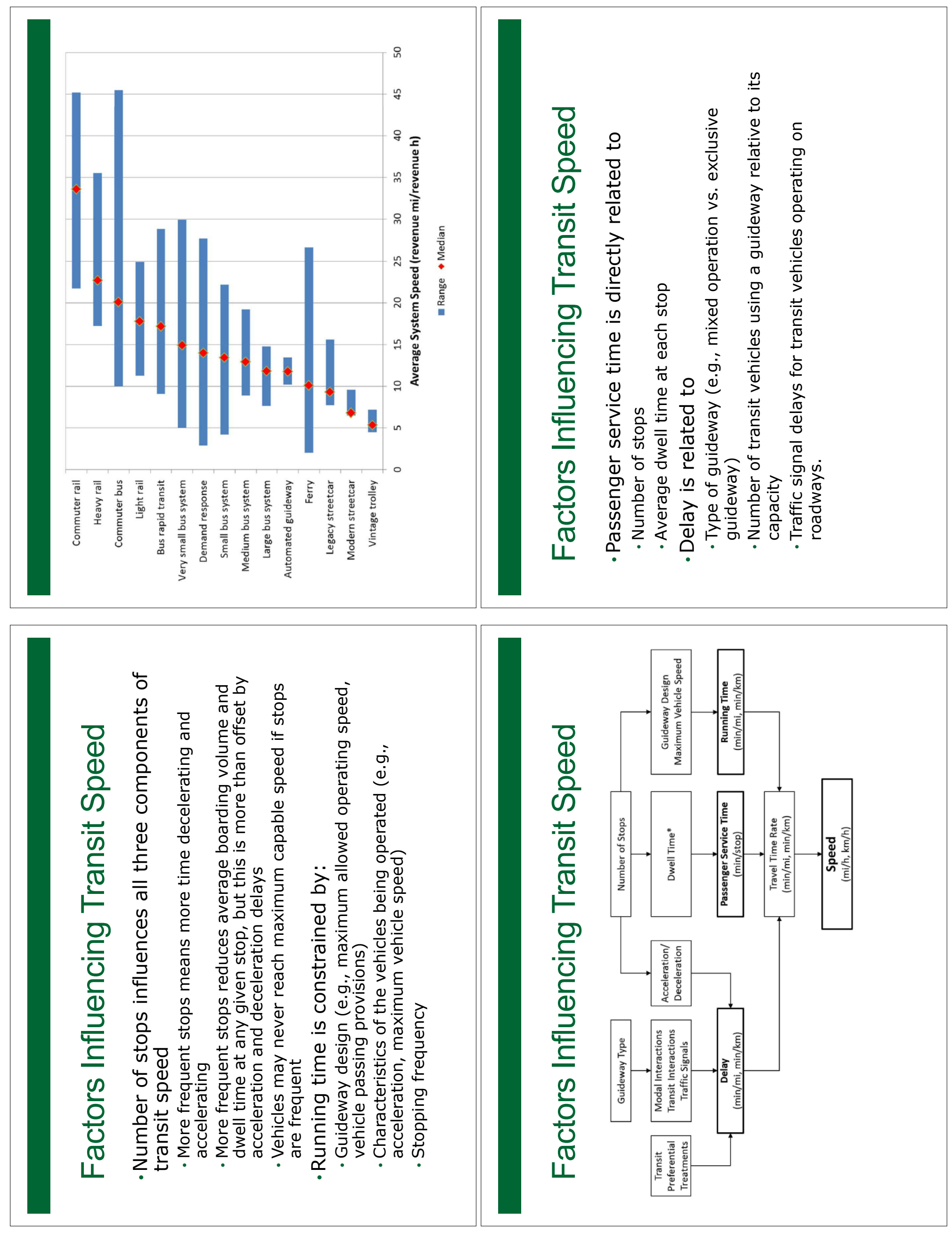

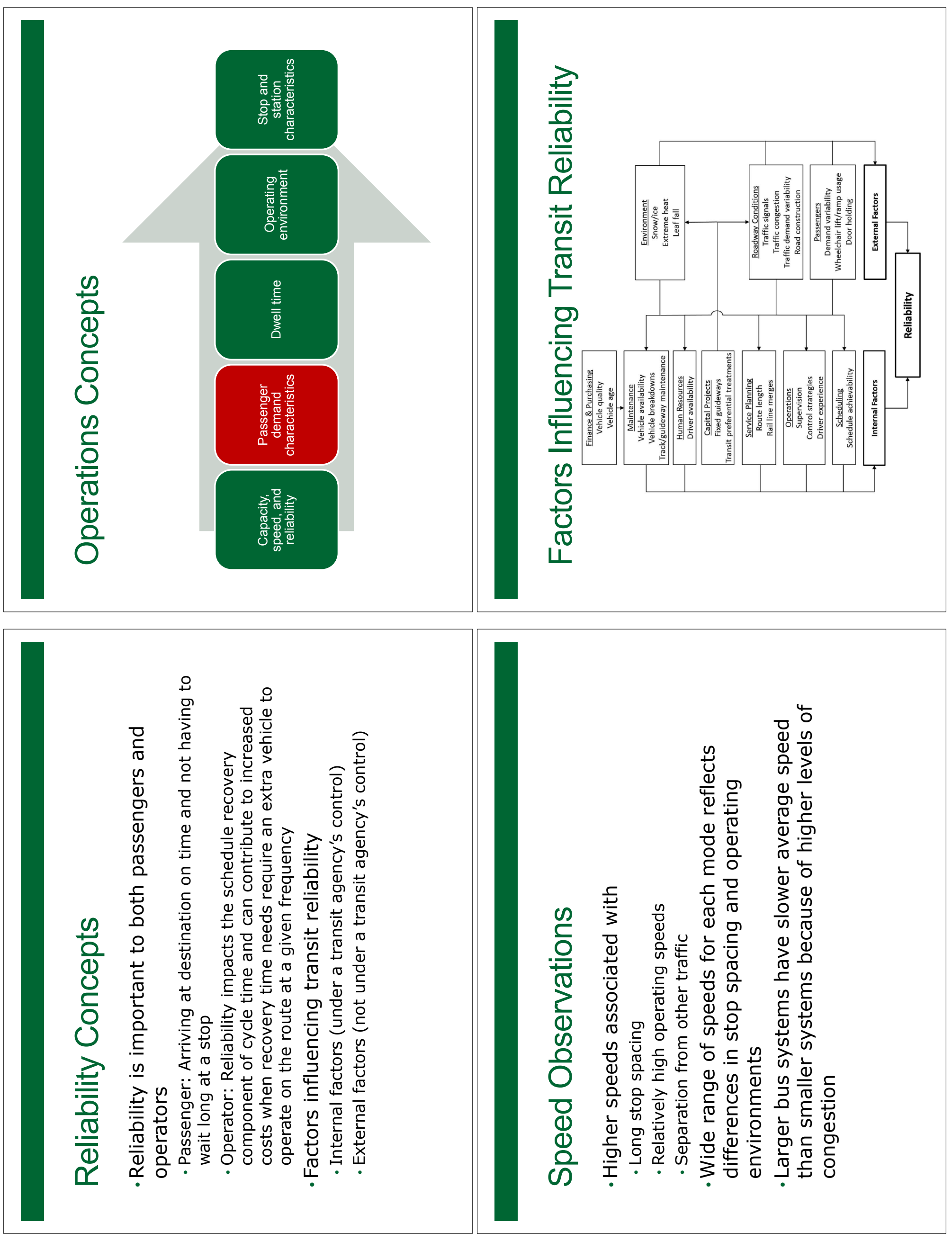

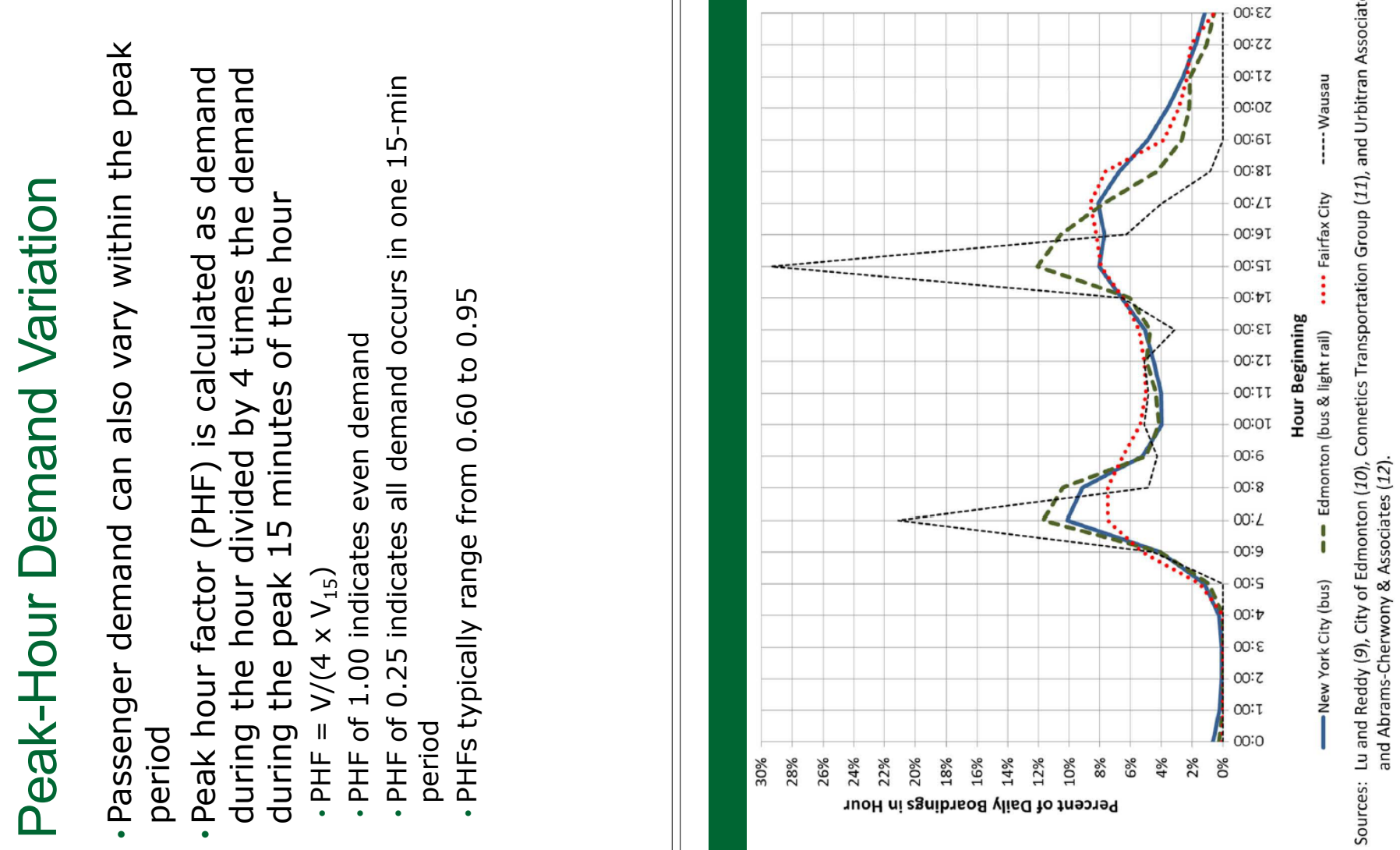

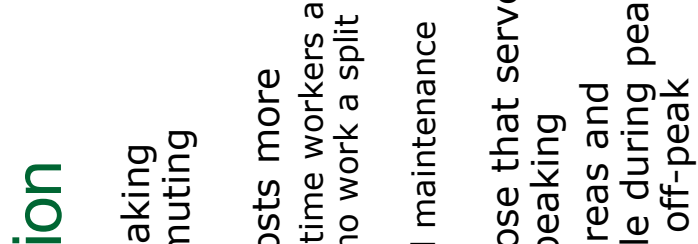

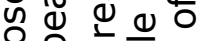

ญह

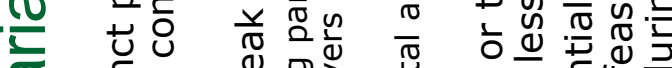

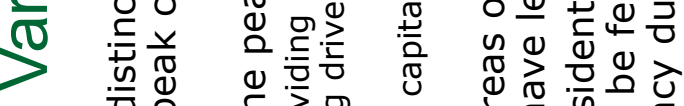

市

(1) $⿻ \pm \frac{1}{\square}$

ט

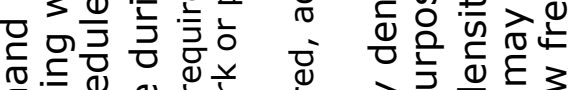

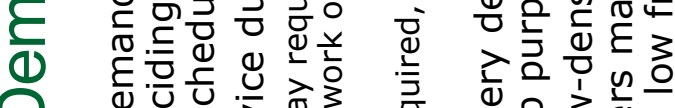

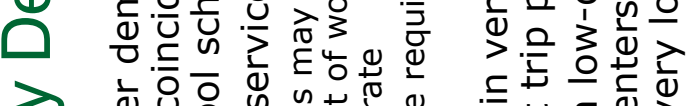

đ)

O

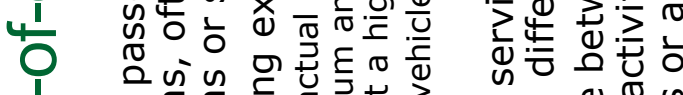

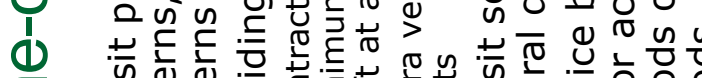

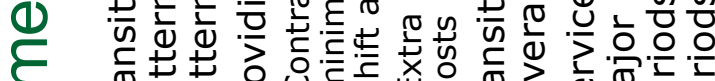

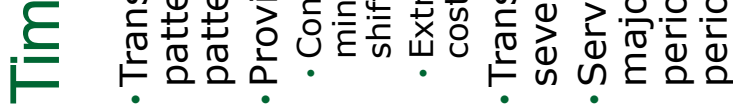

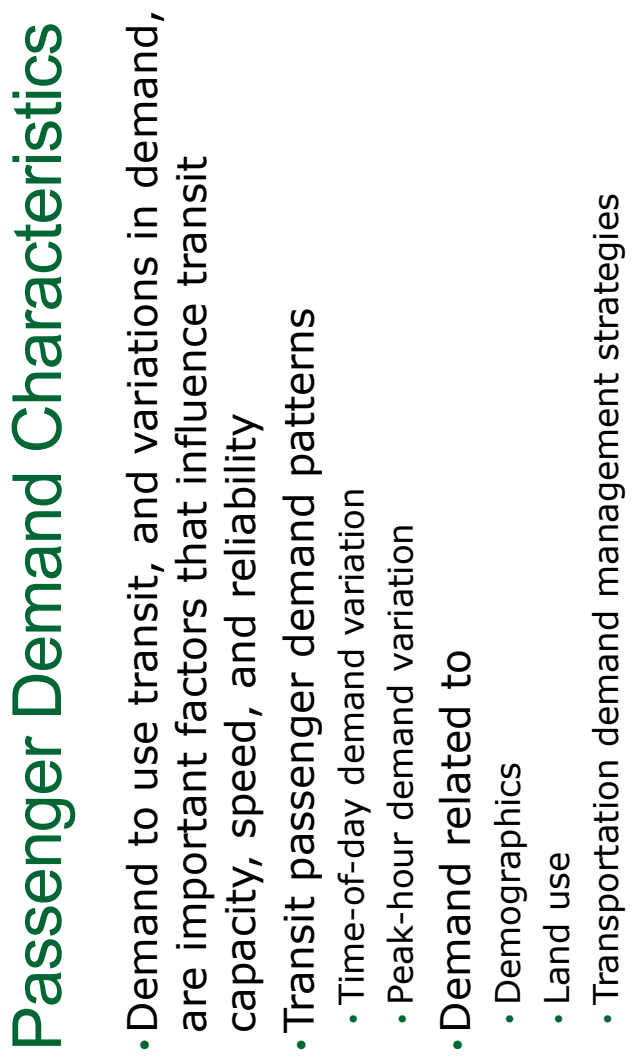



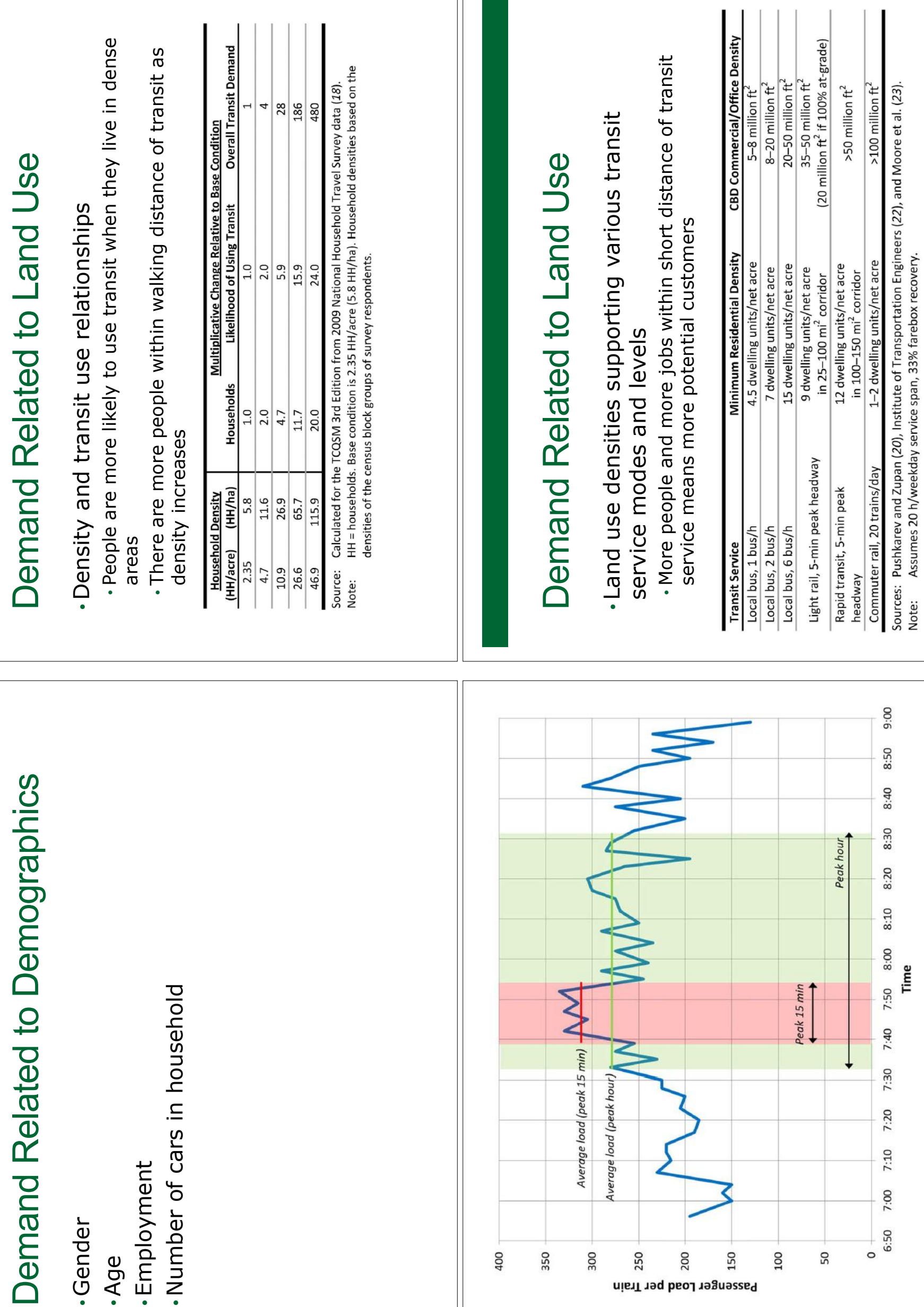

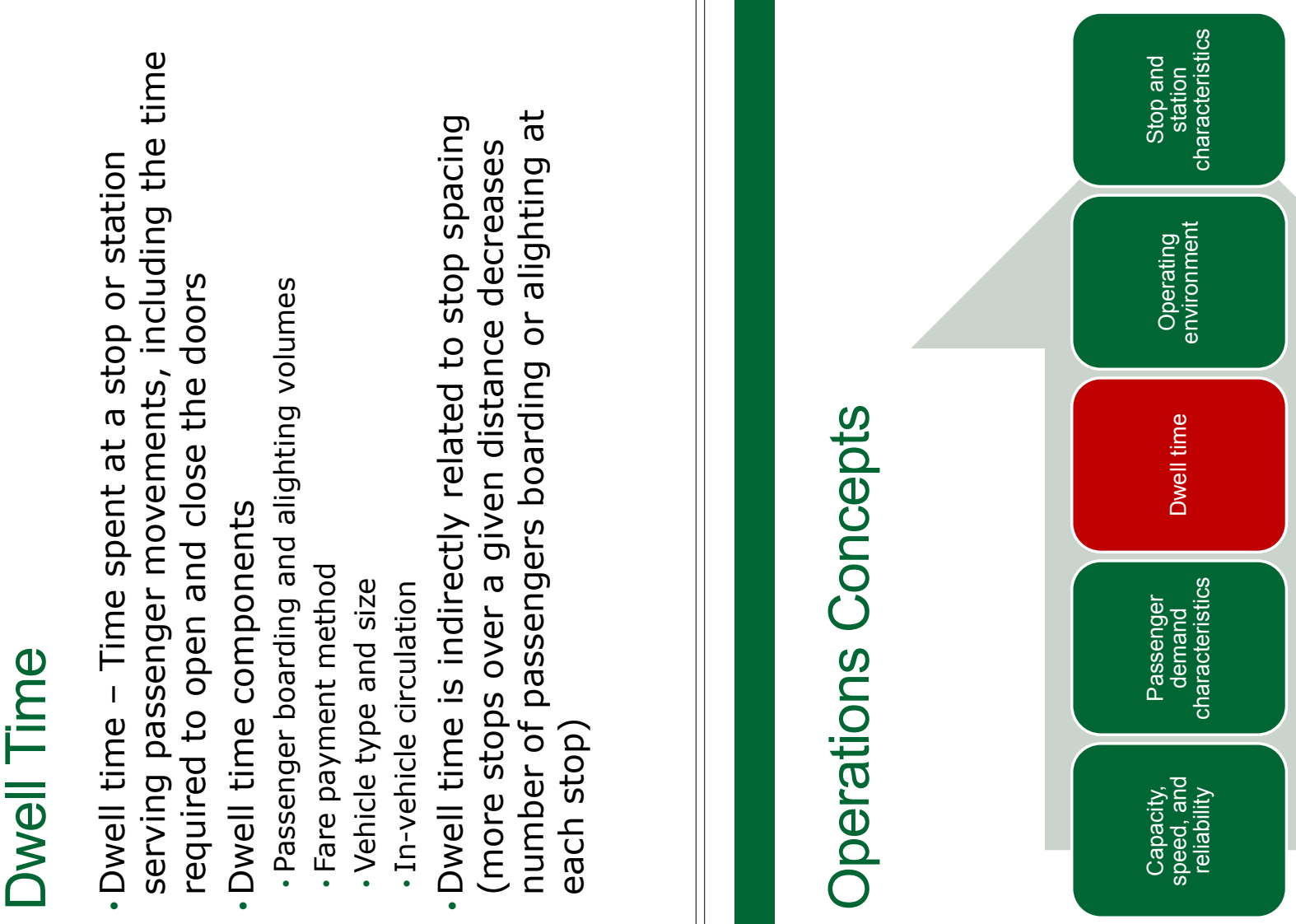

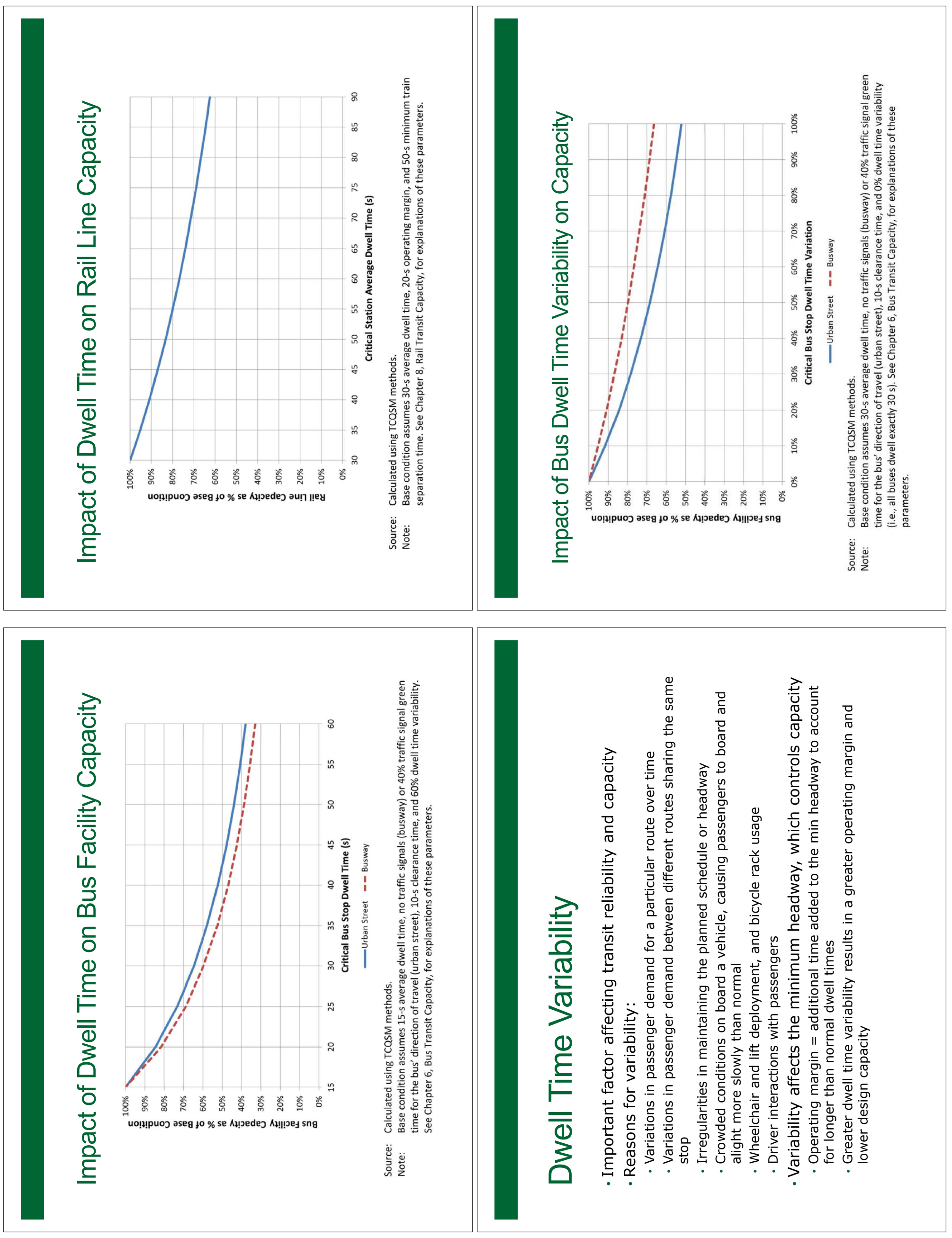

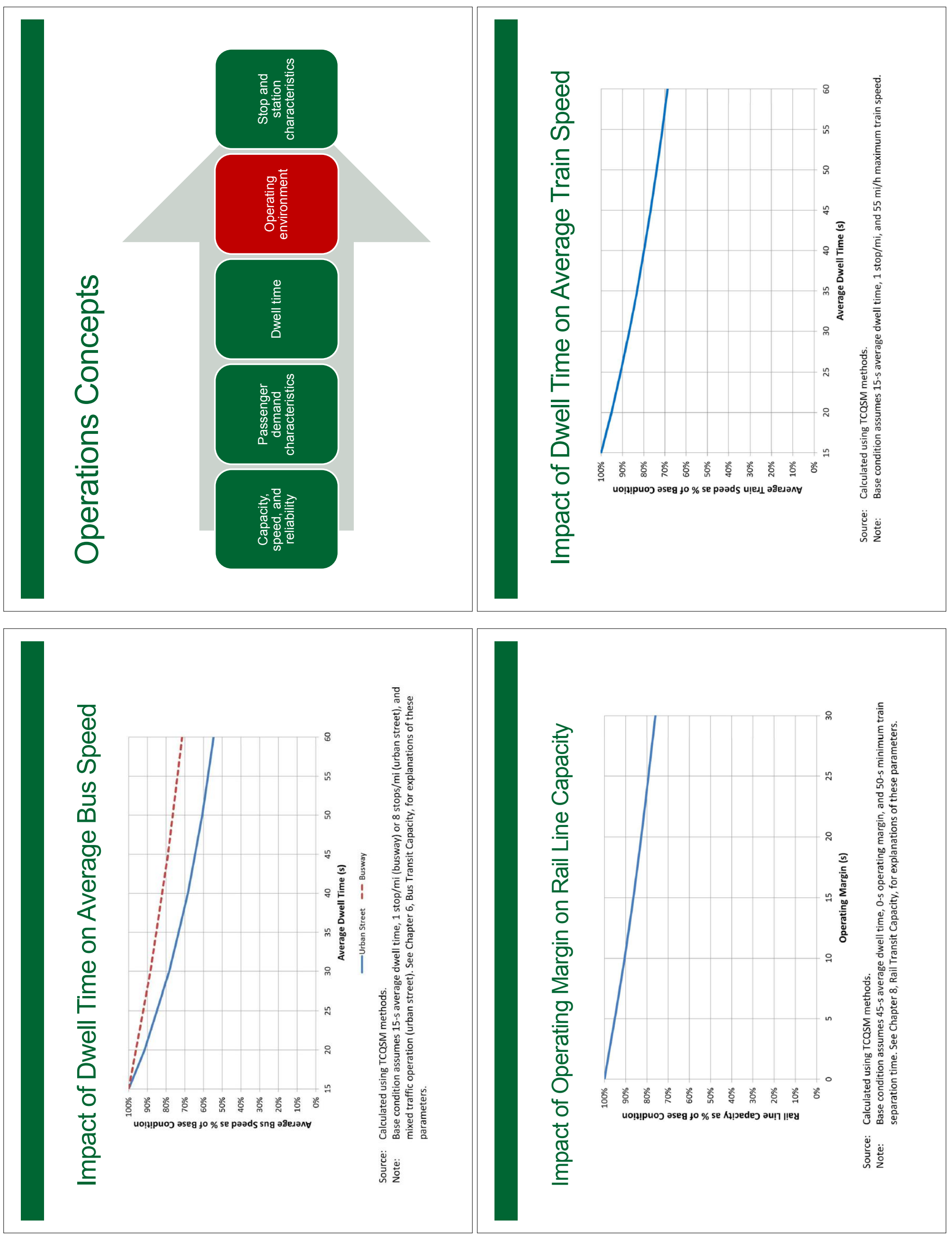

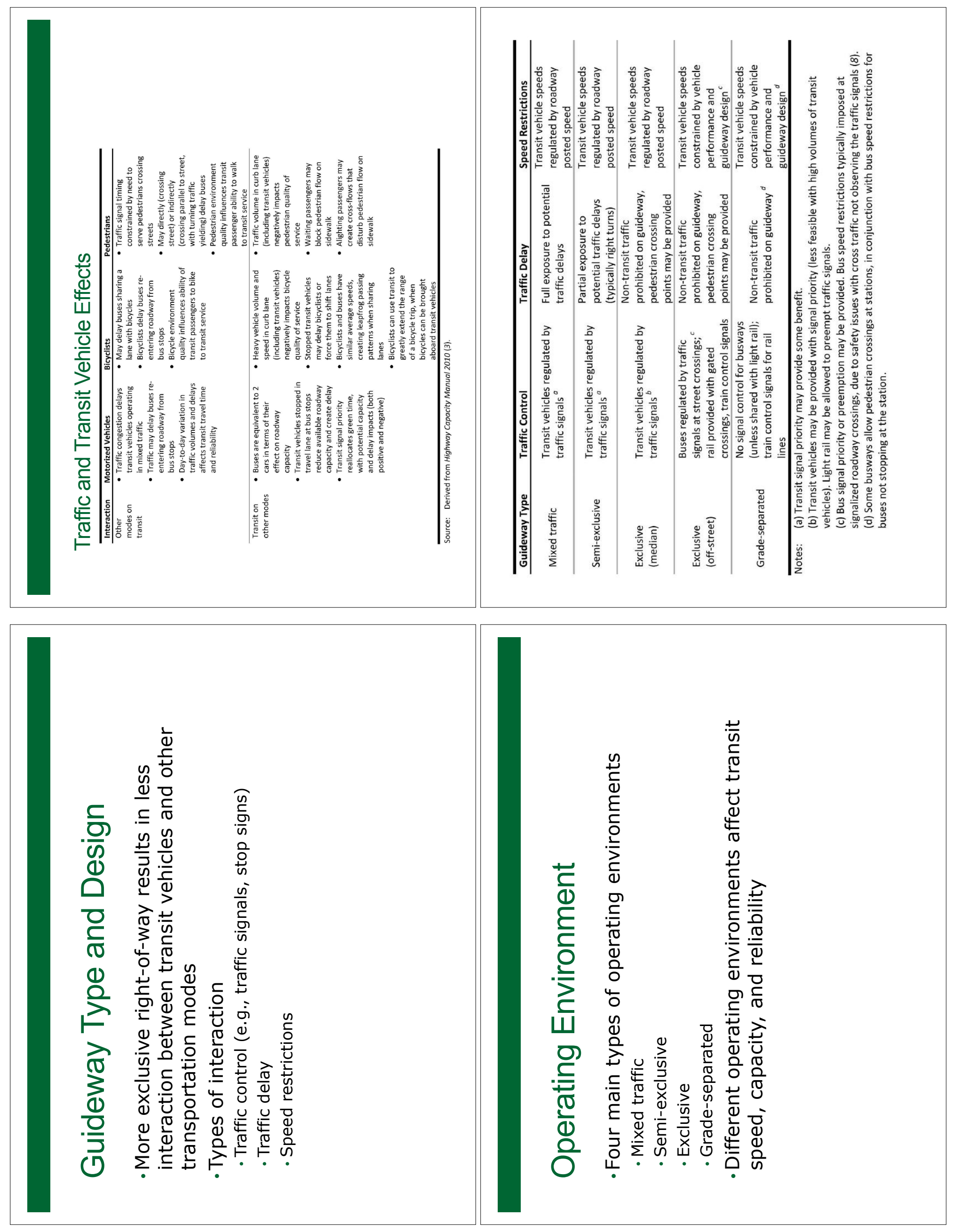

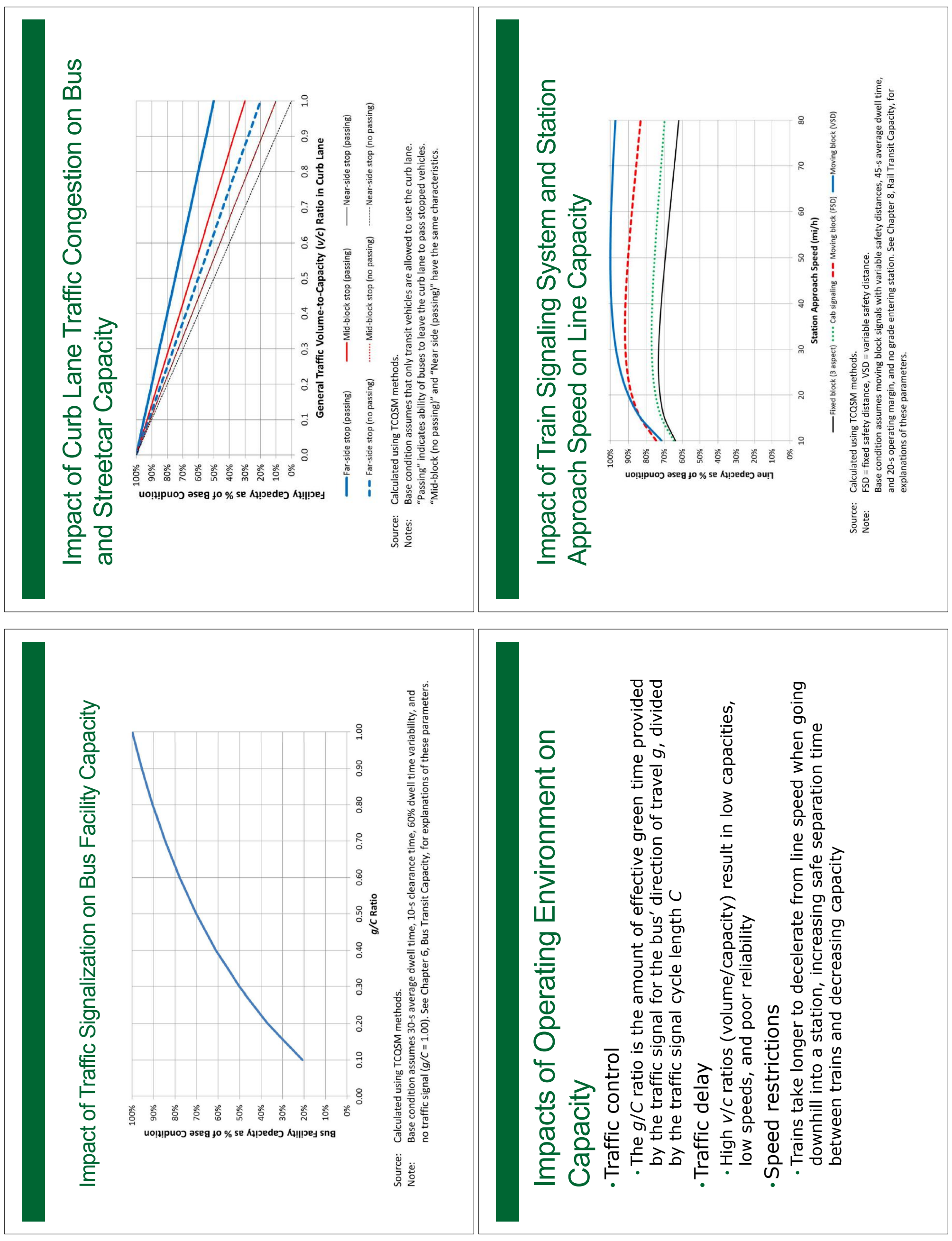

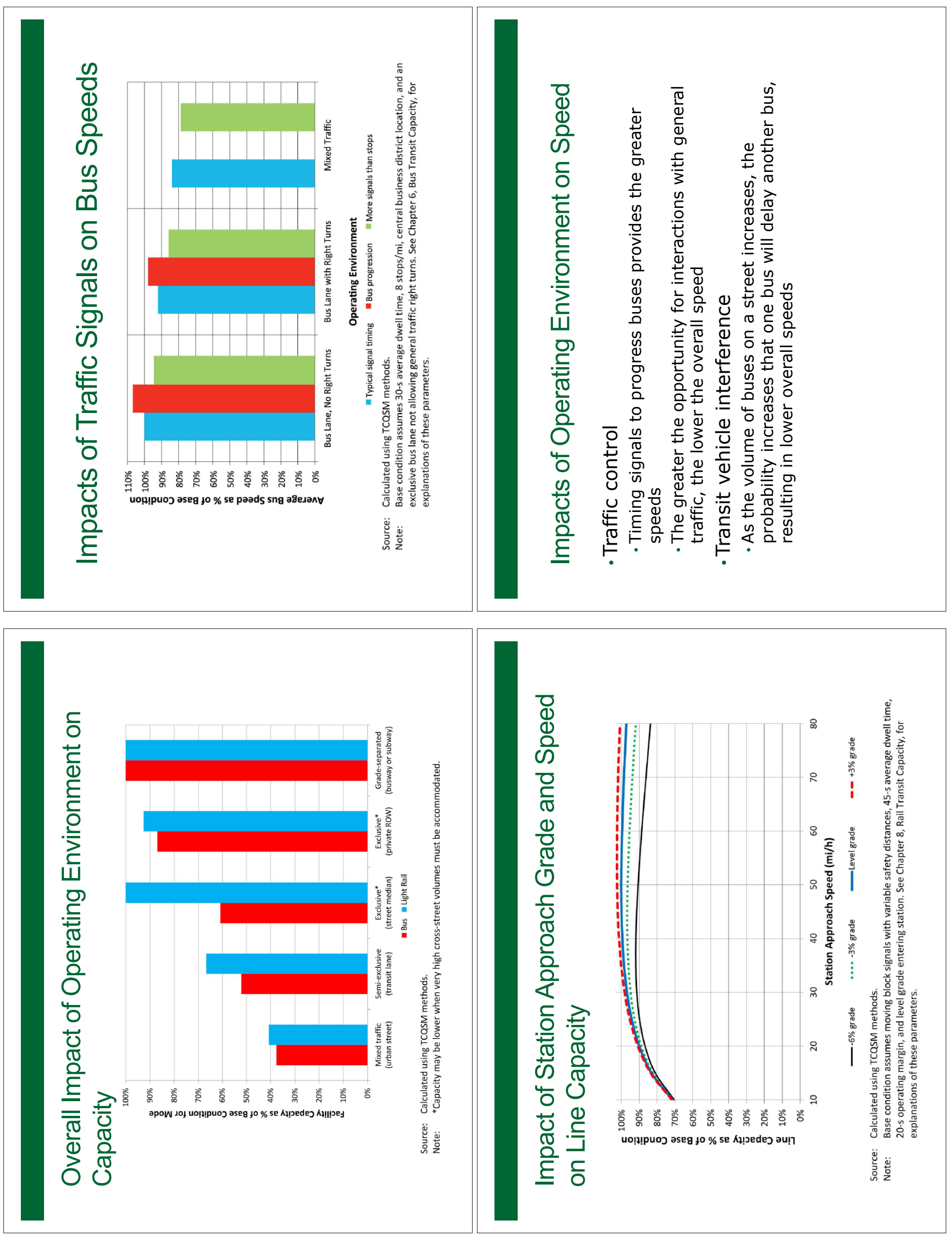

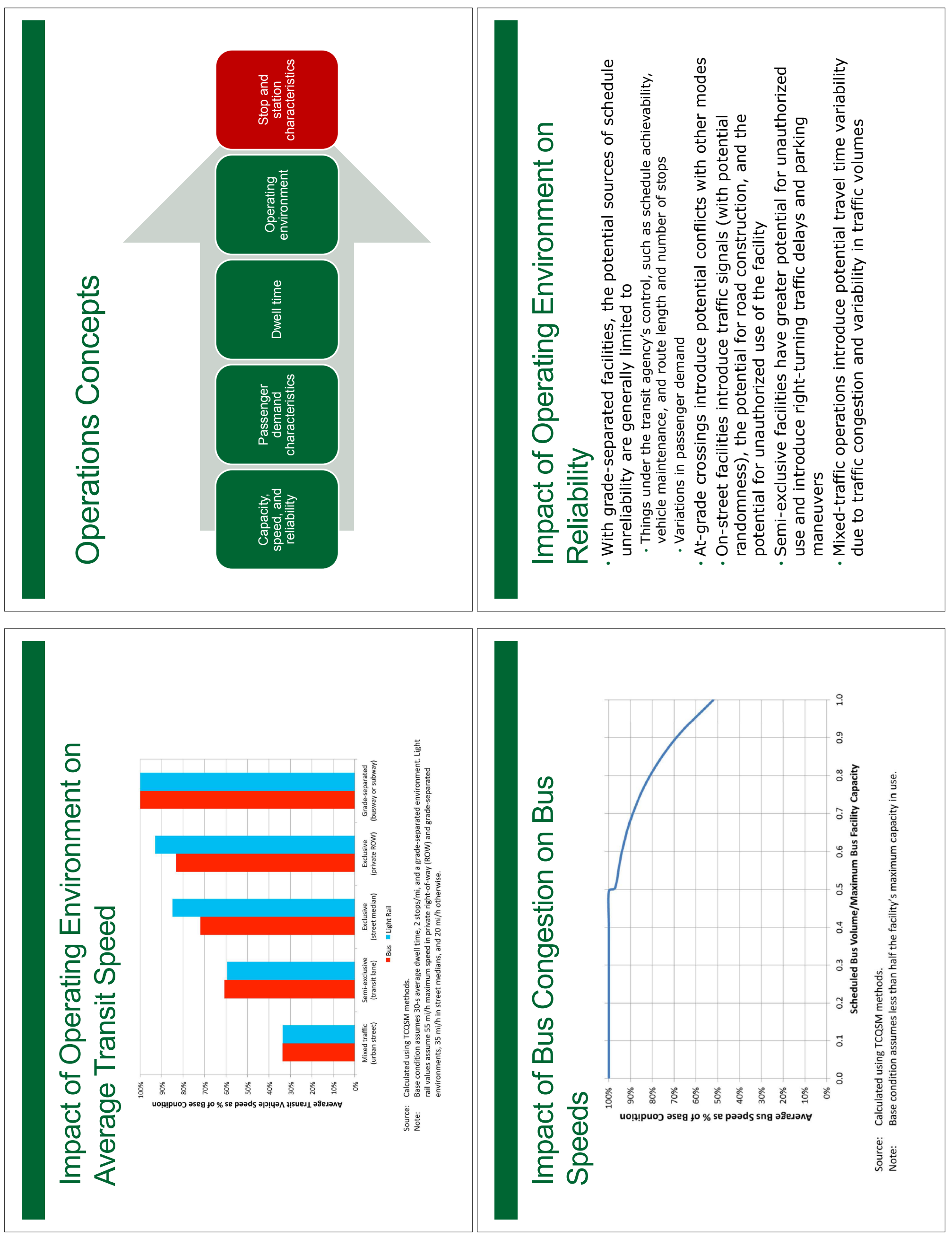

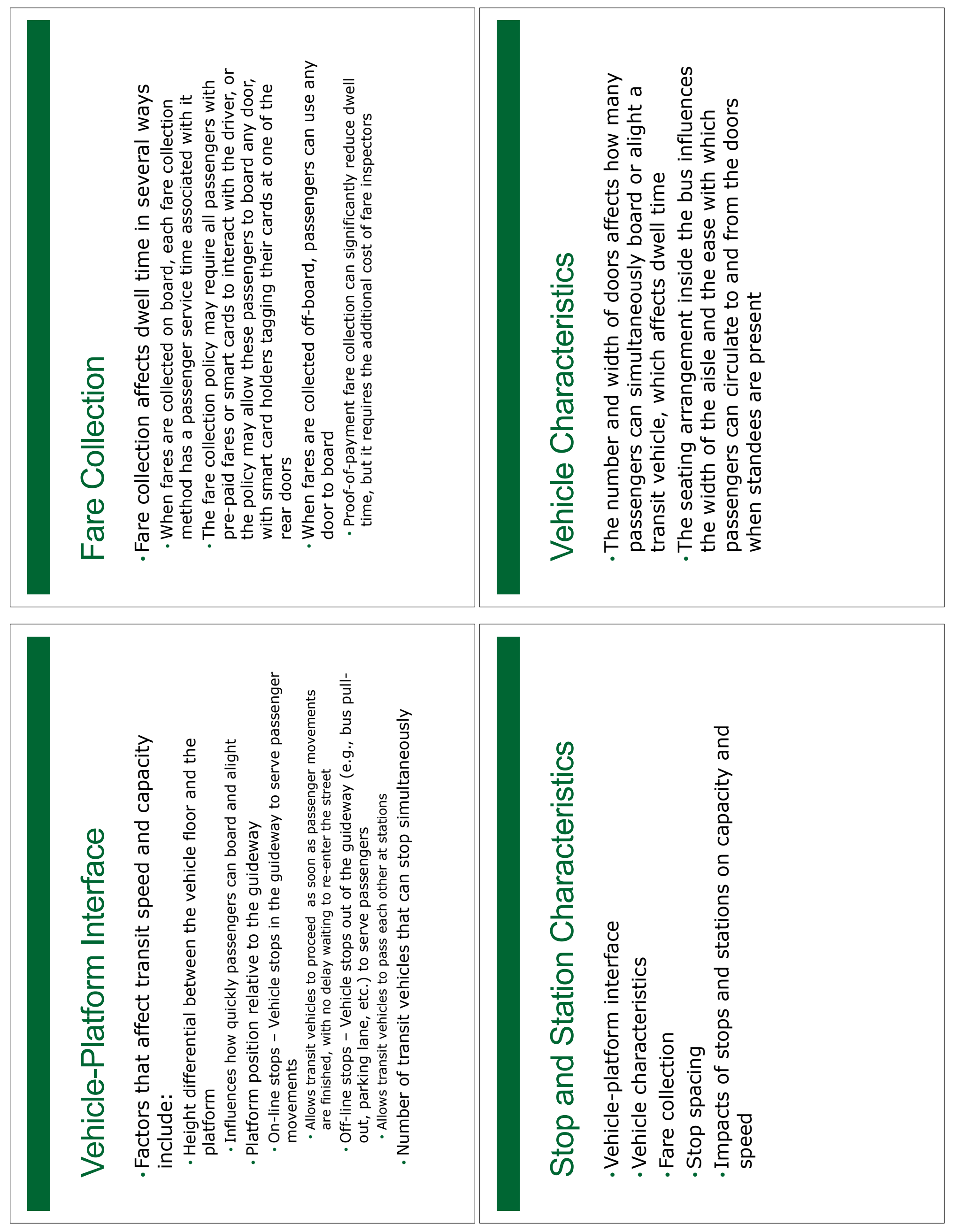

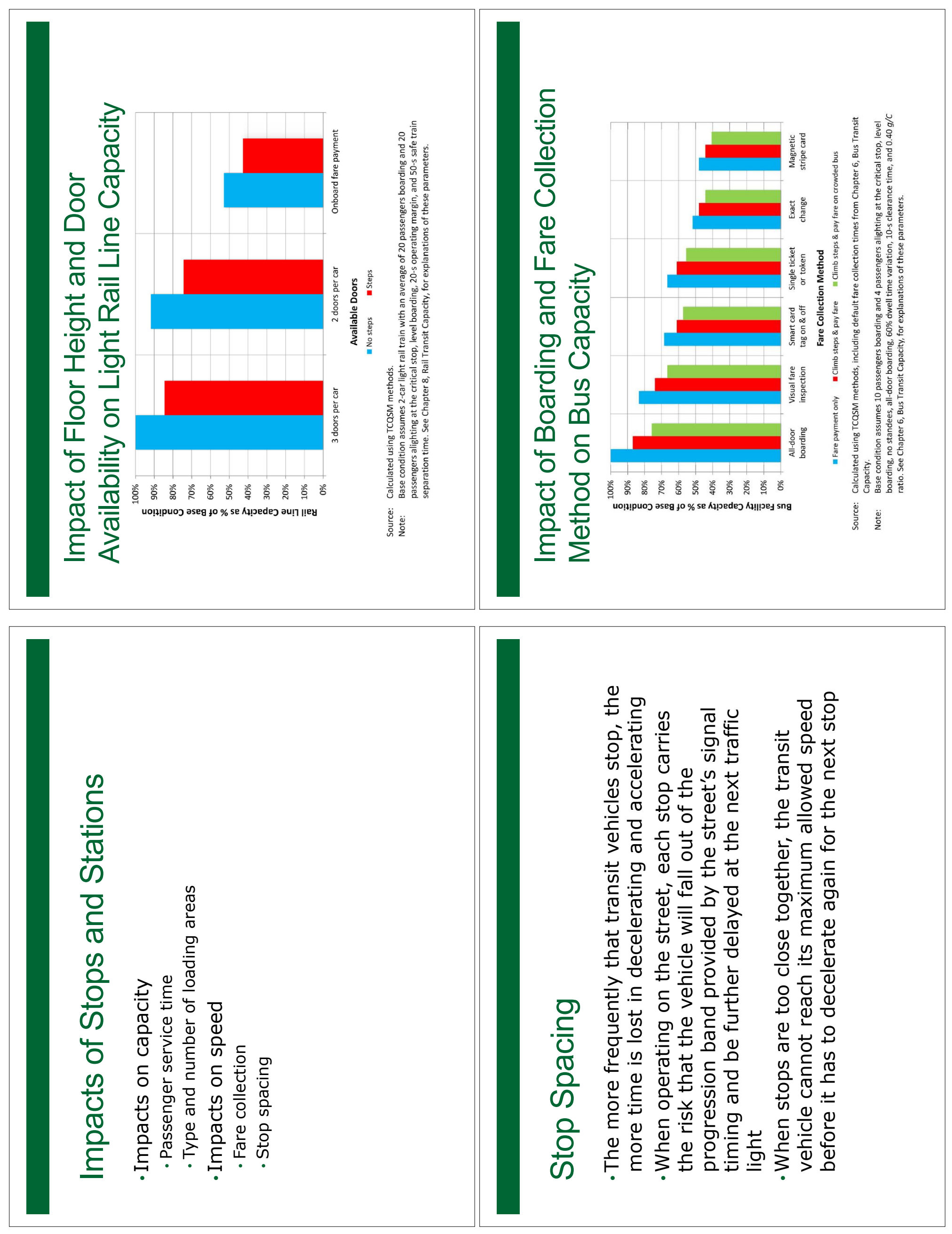

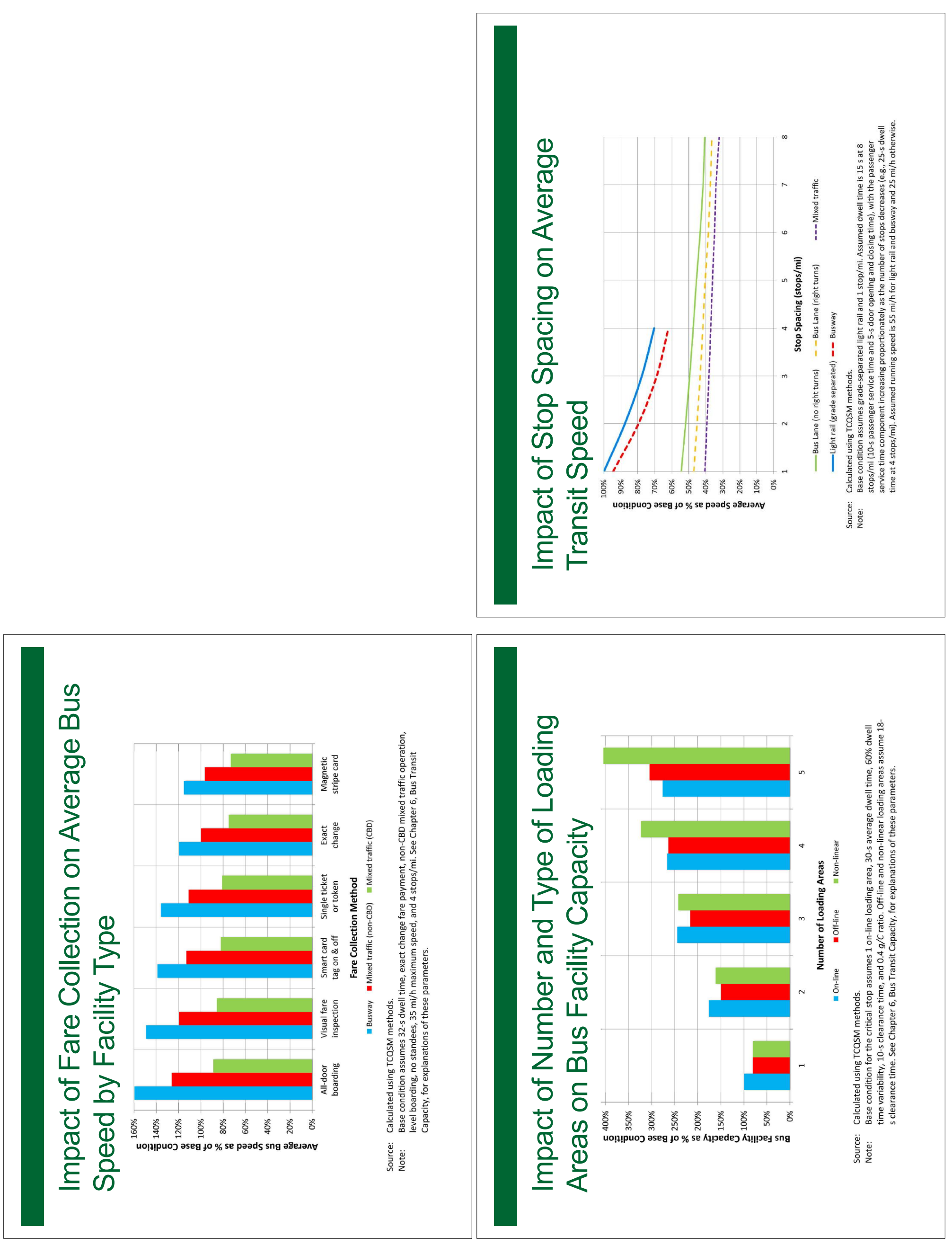

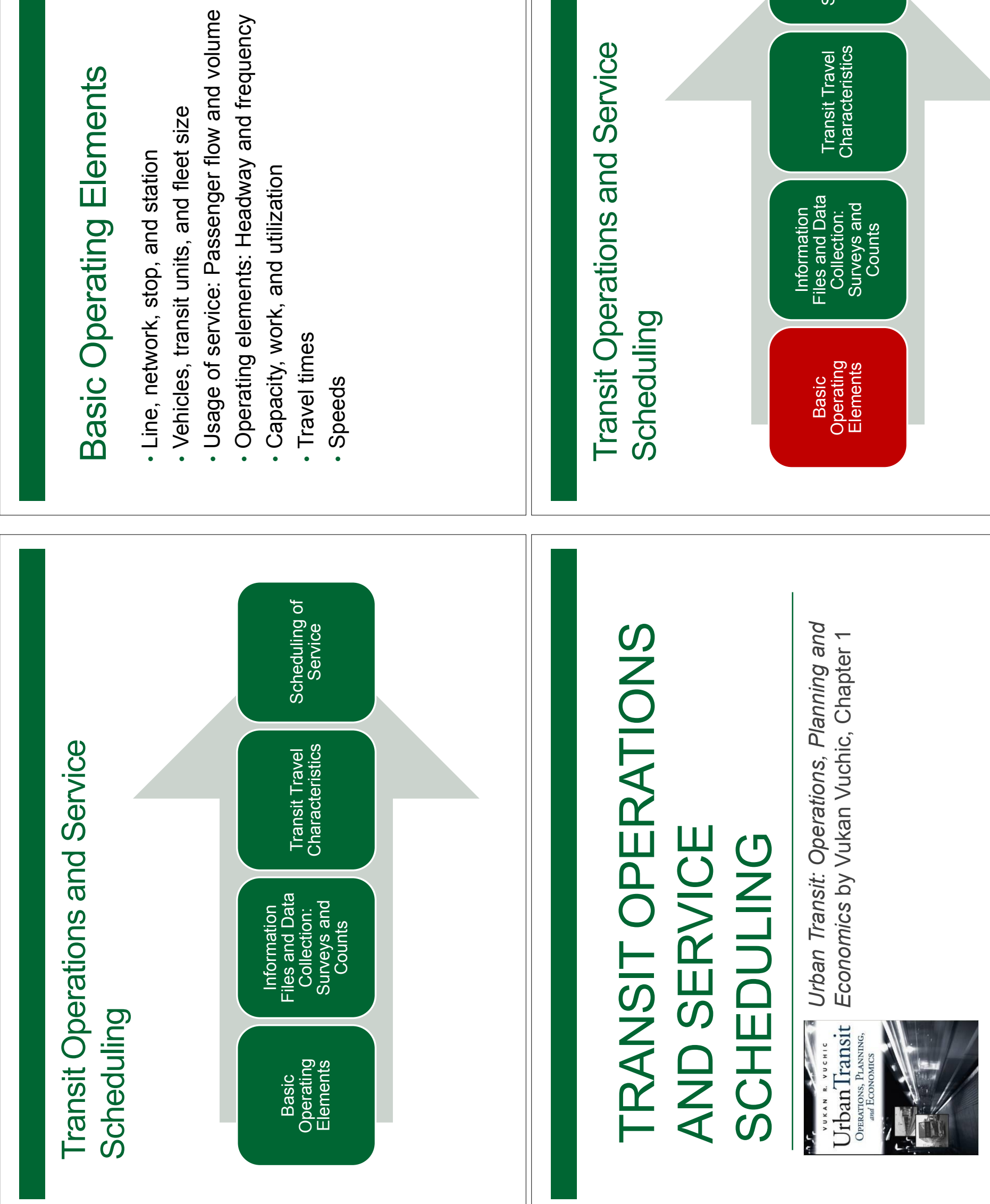

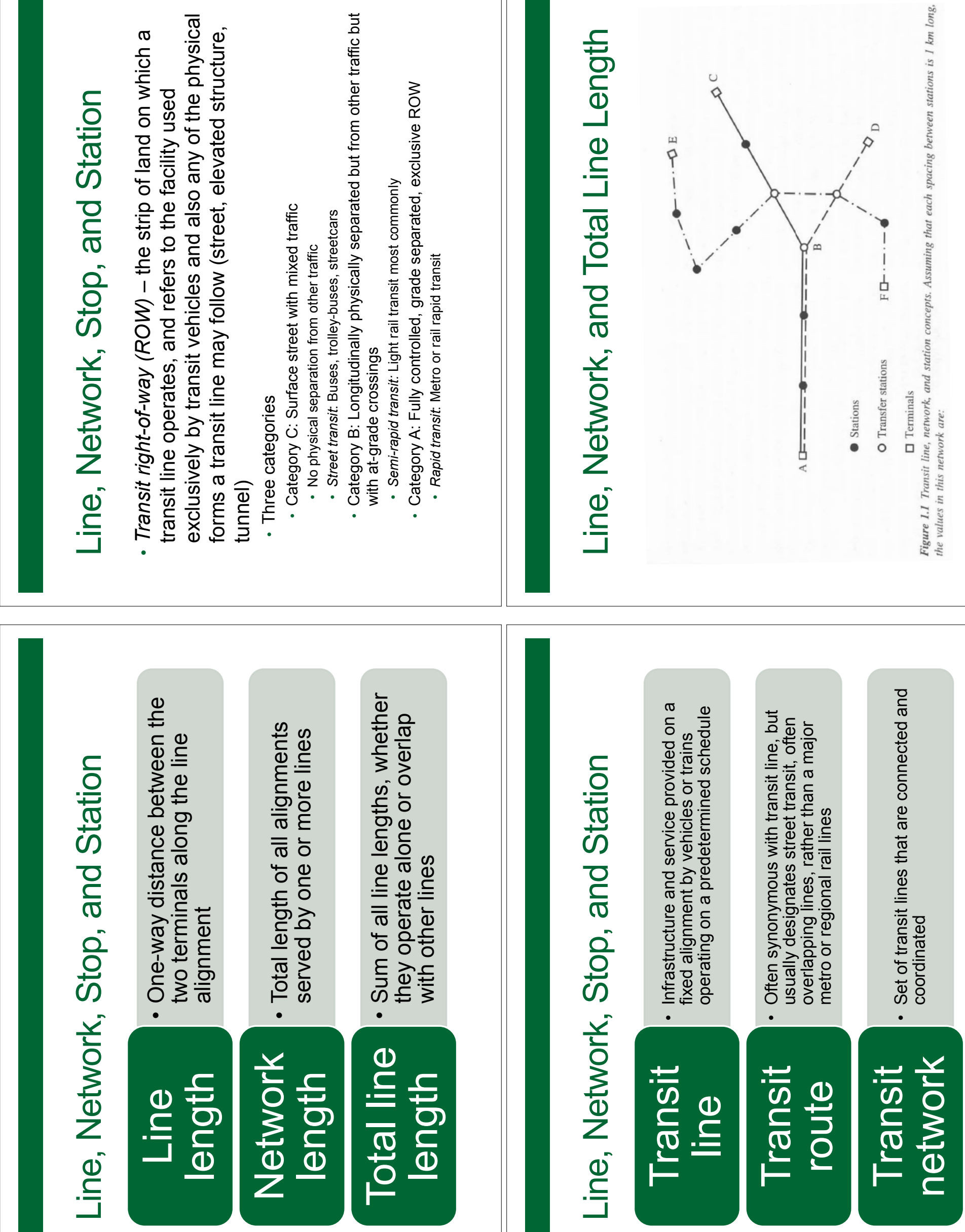

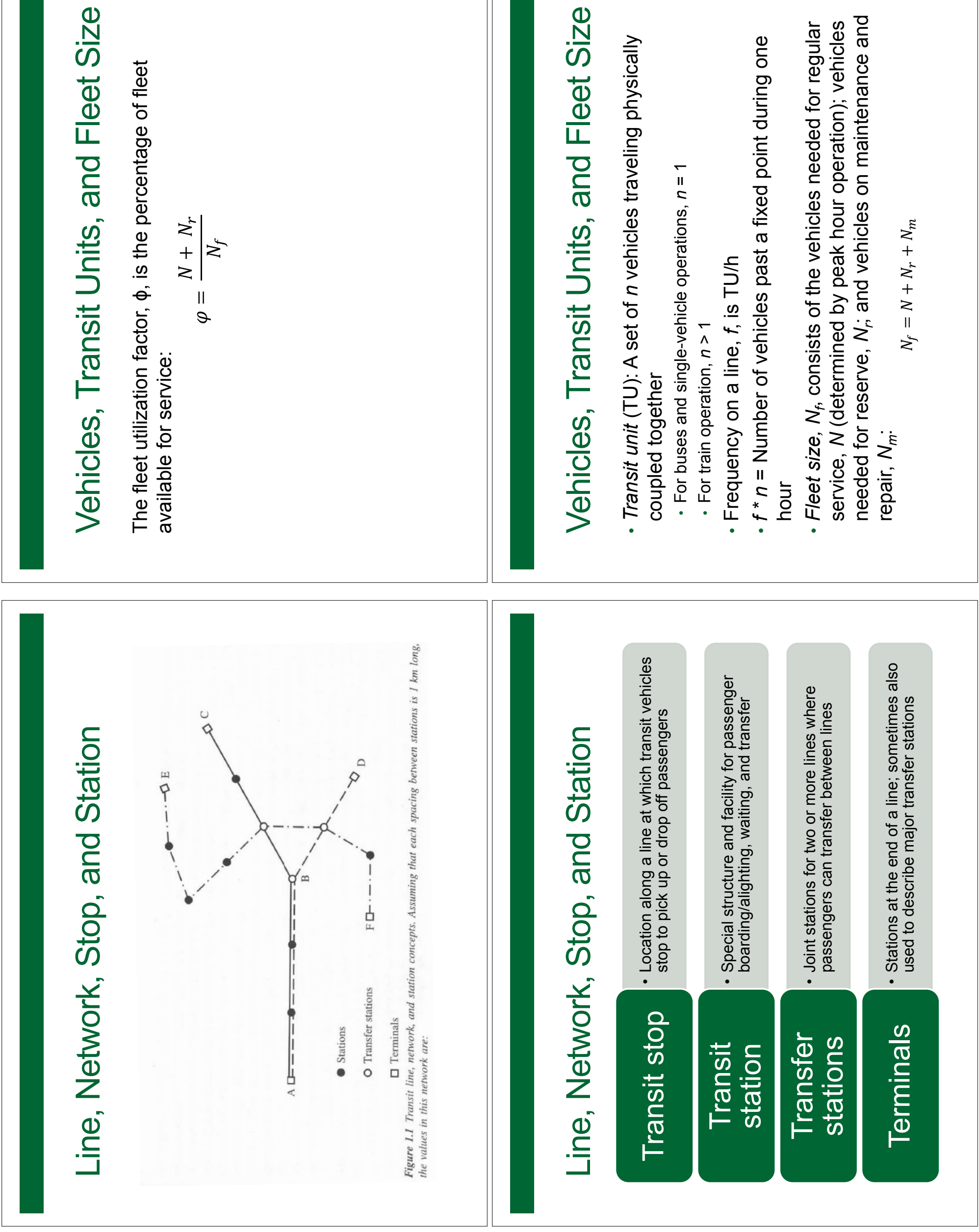

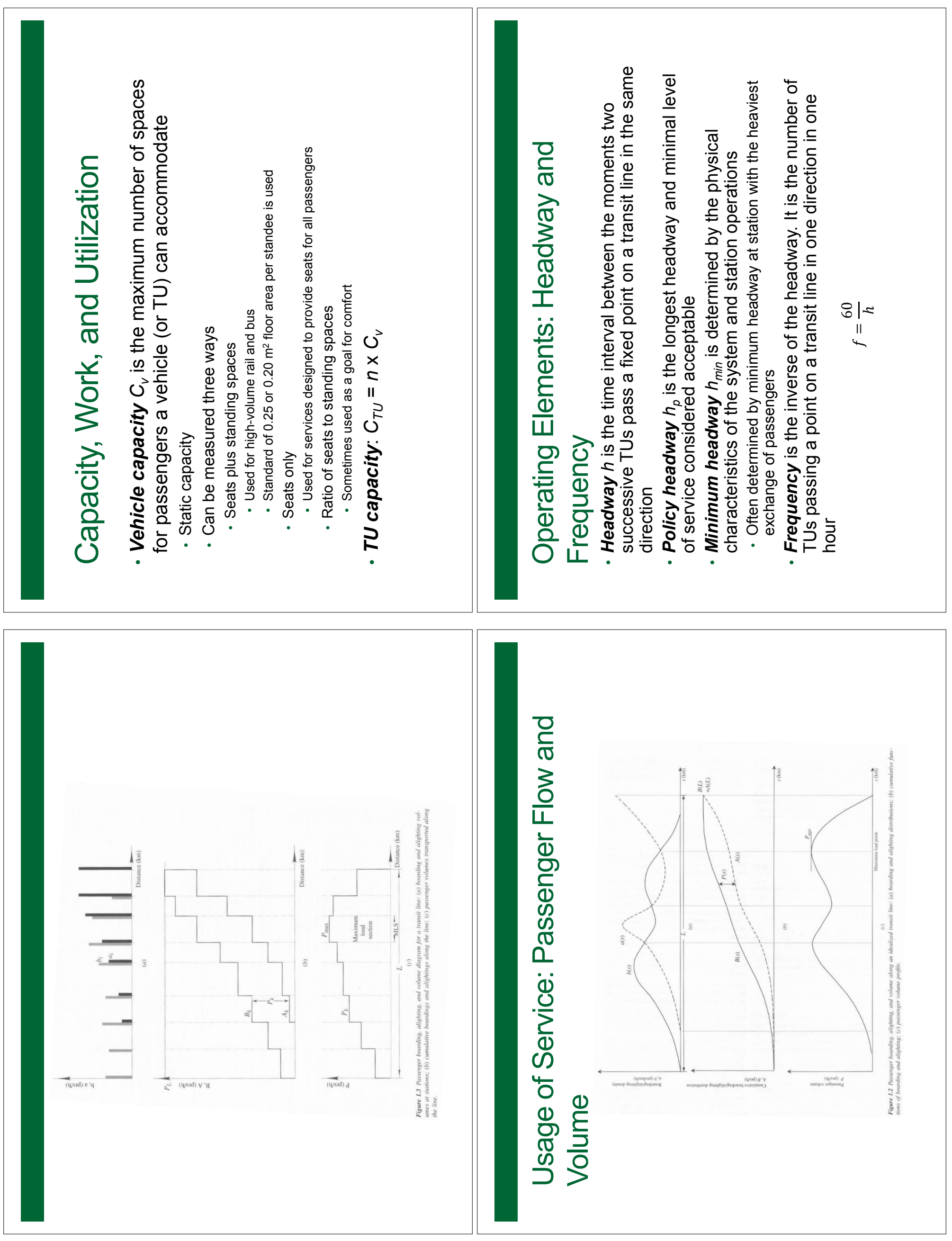


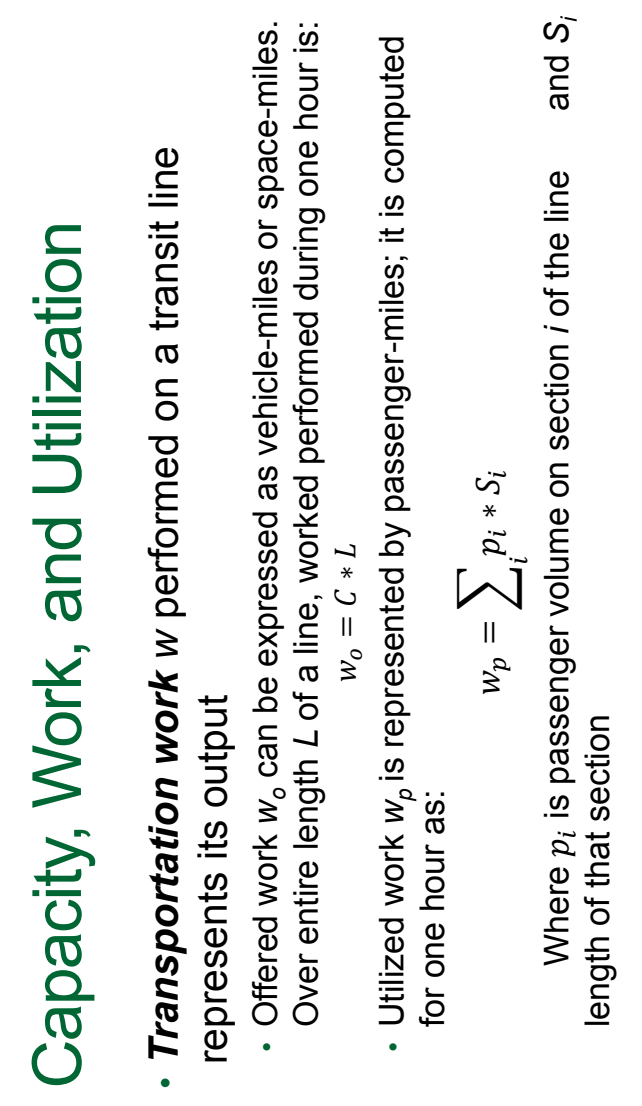

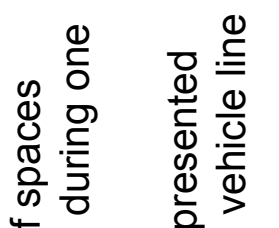

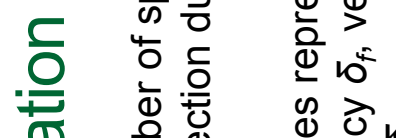

สে

Nㅡㄹ

言 $\quad 0$ 중

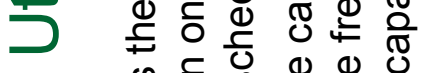

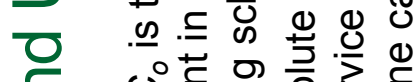

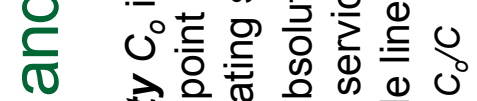

-

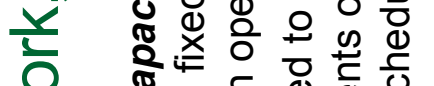

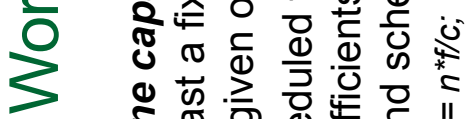

$>\quad \stackrel{0}{\equiv}$ 范

文 d d

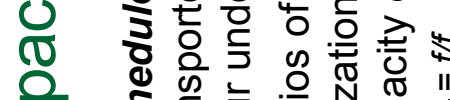

U ত
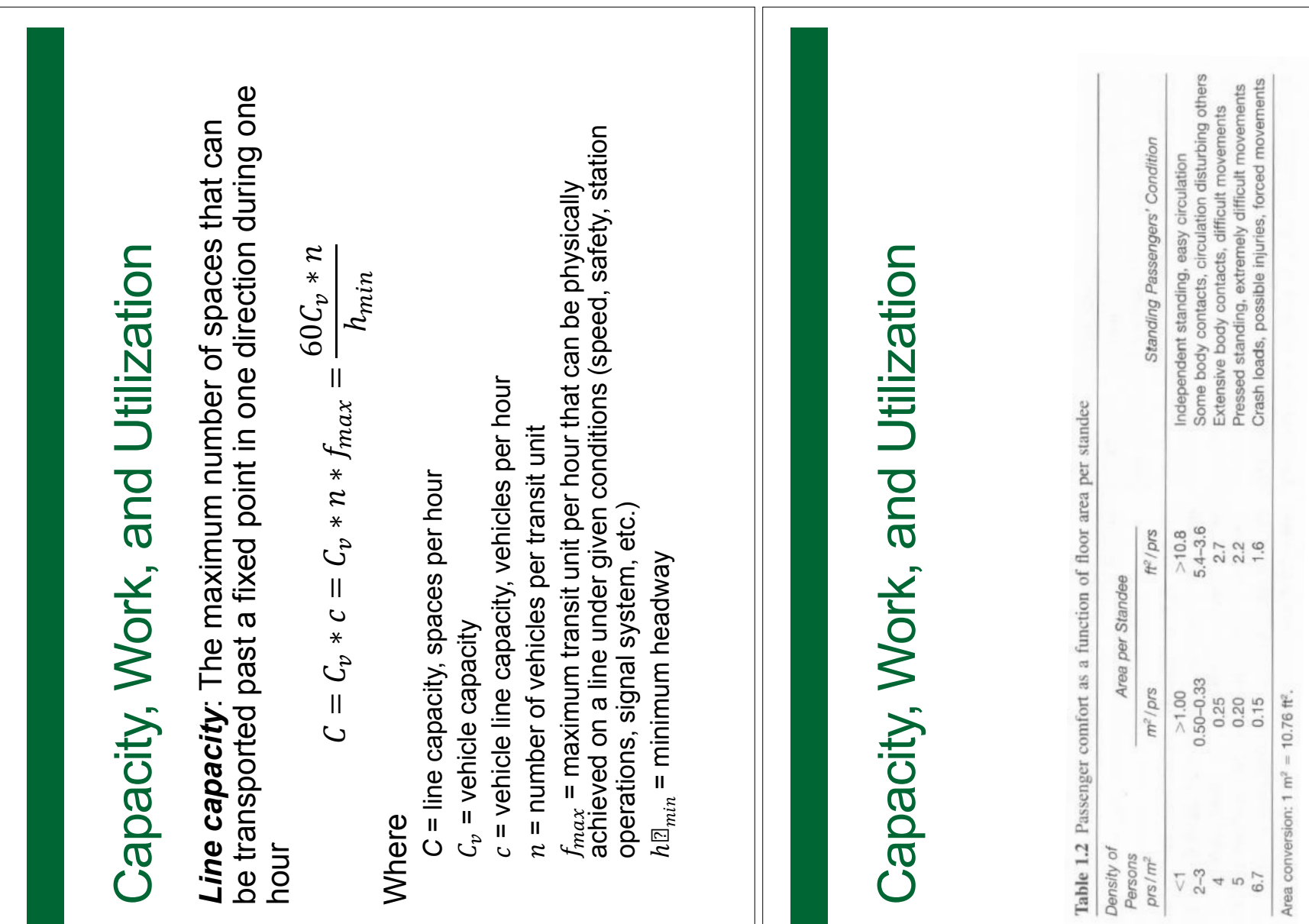

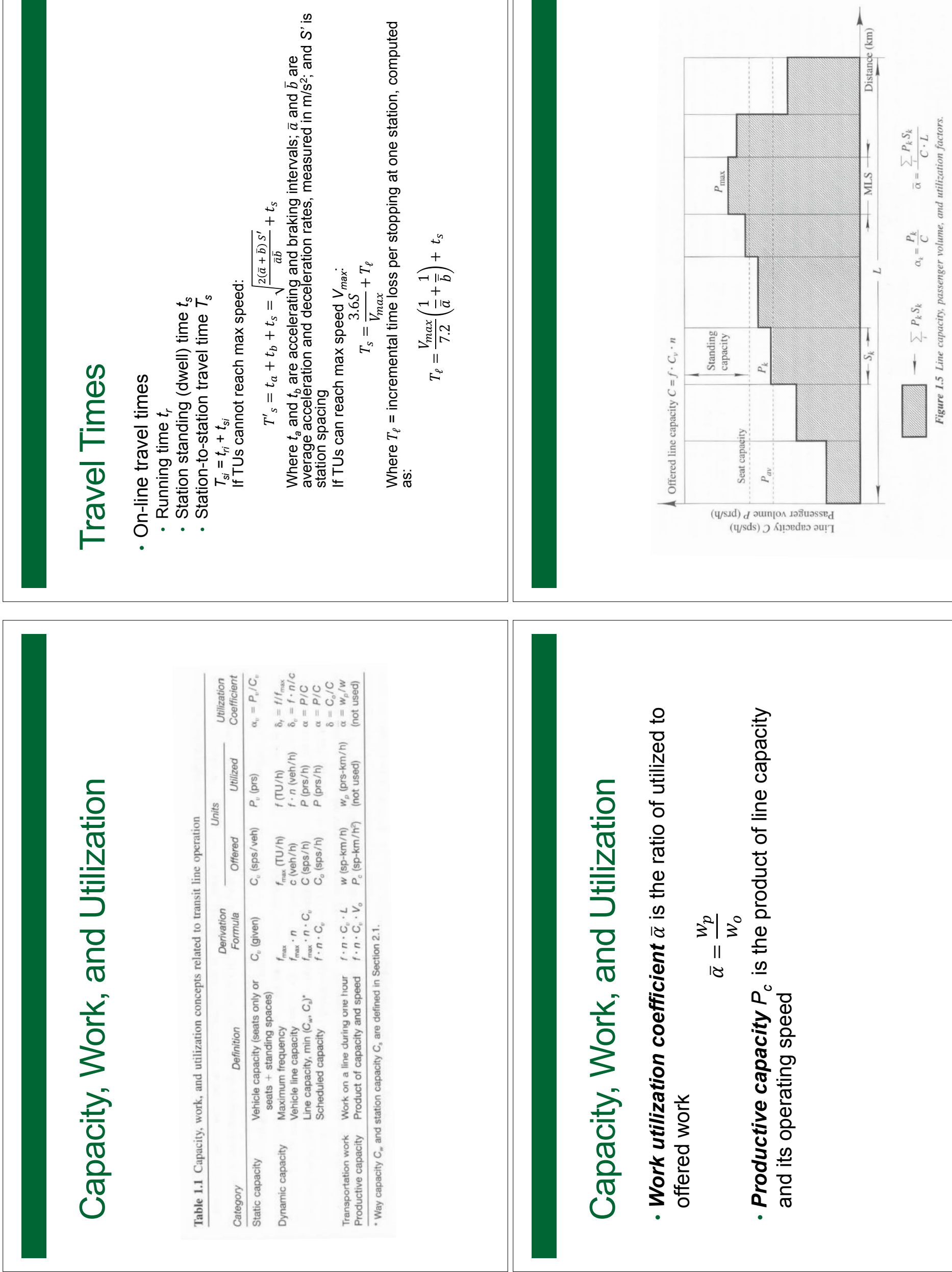

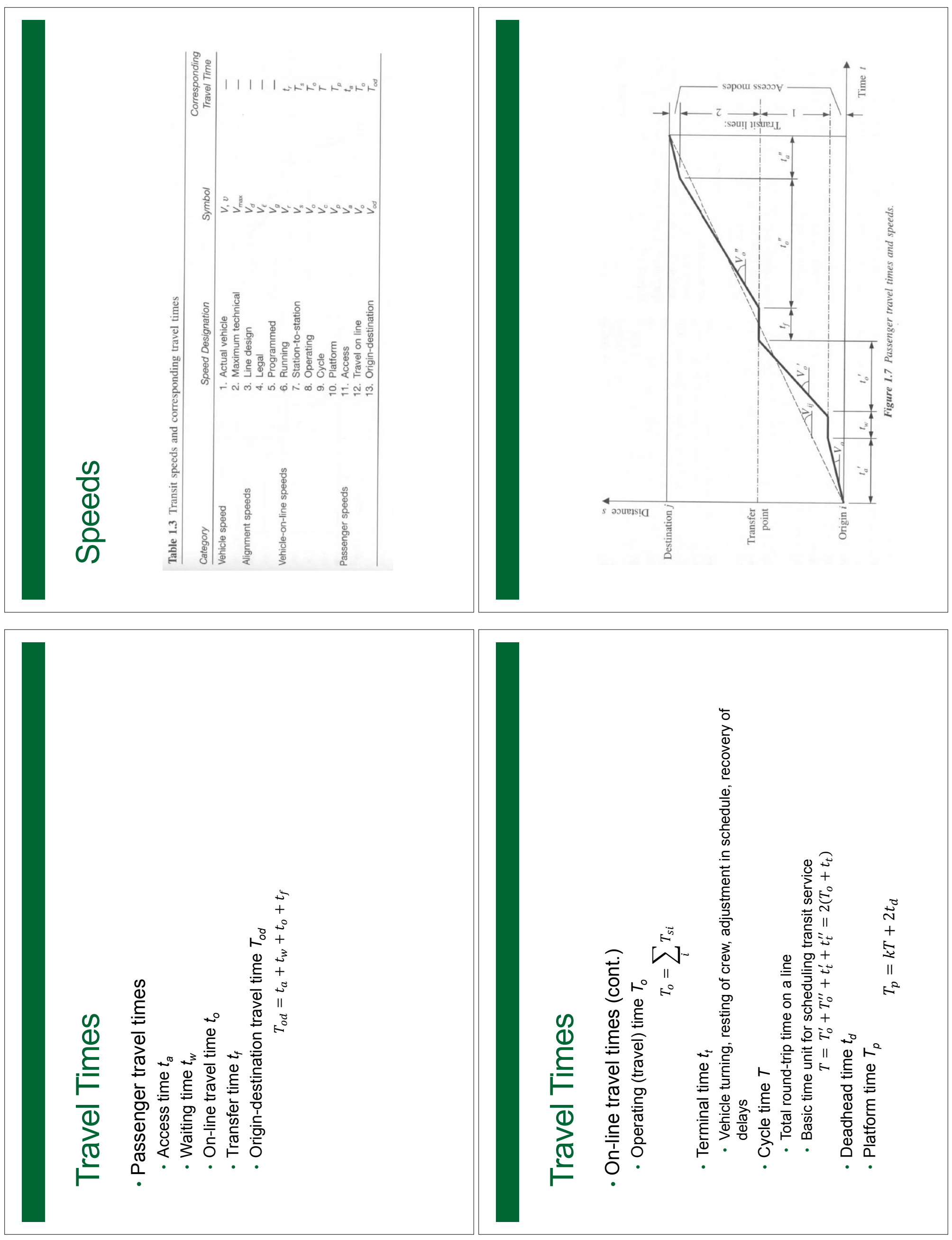

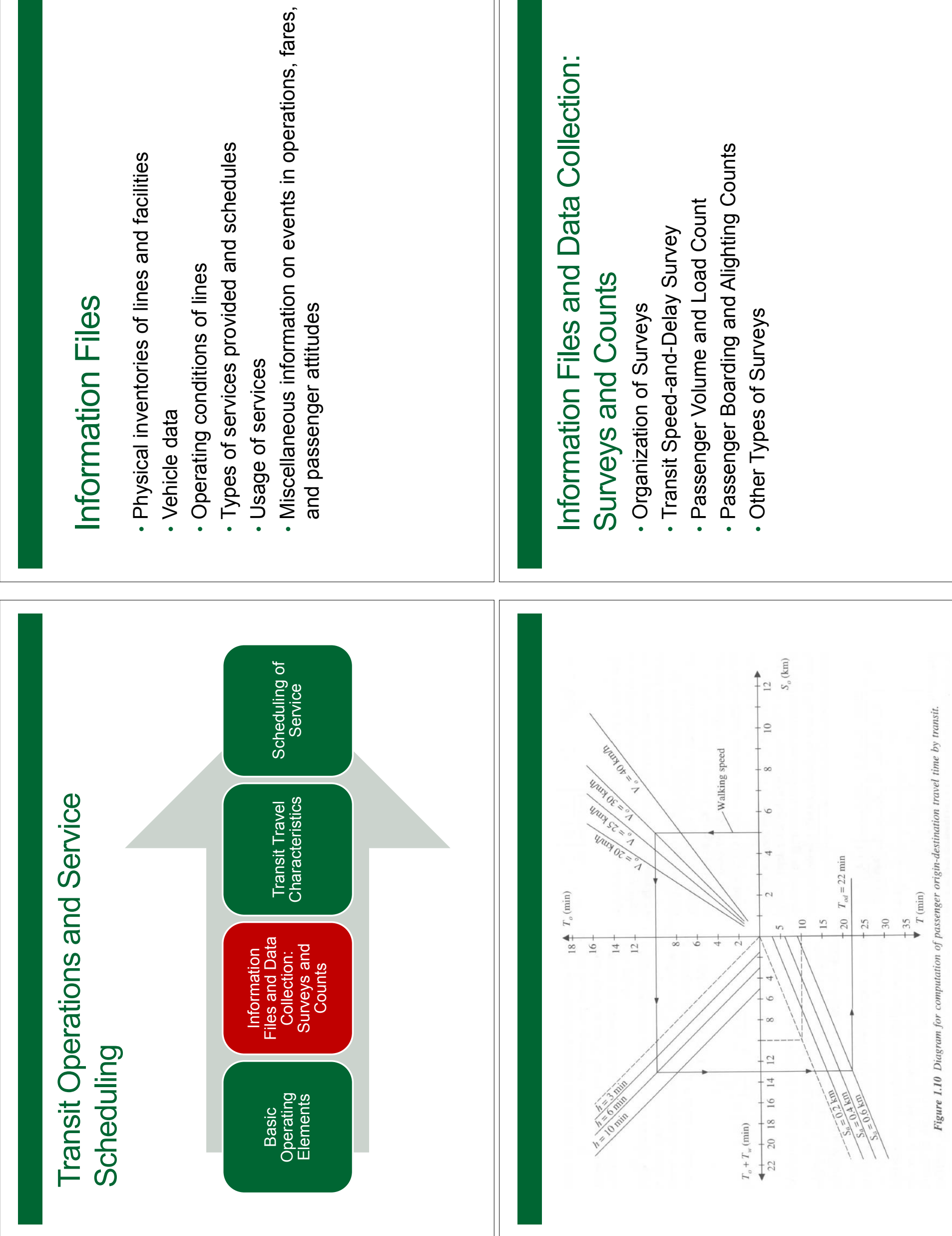

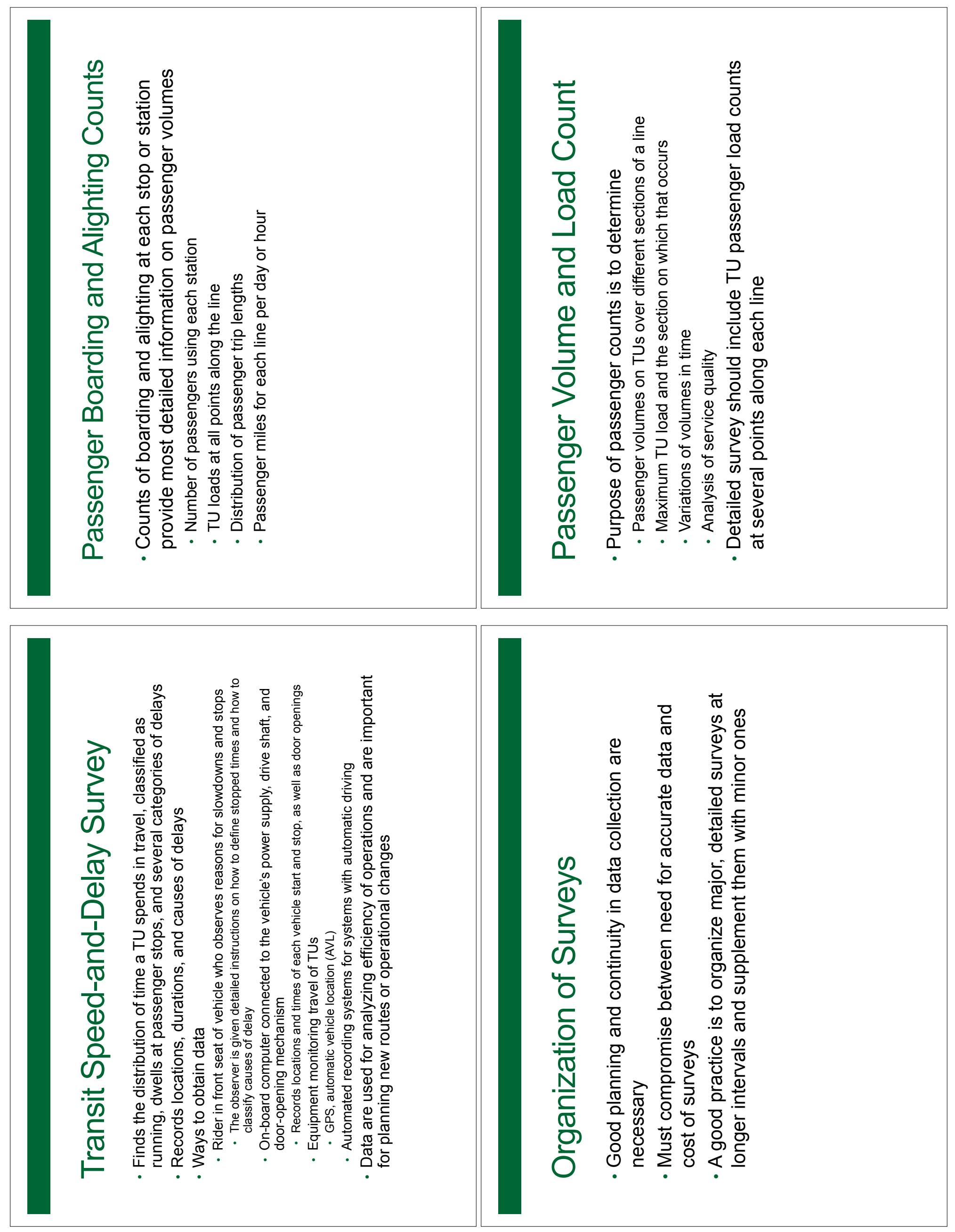

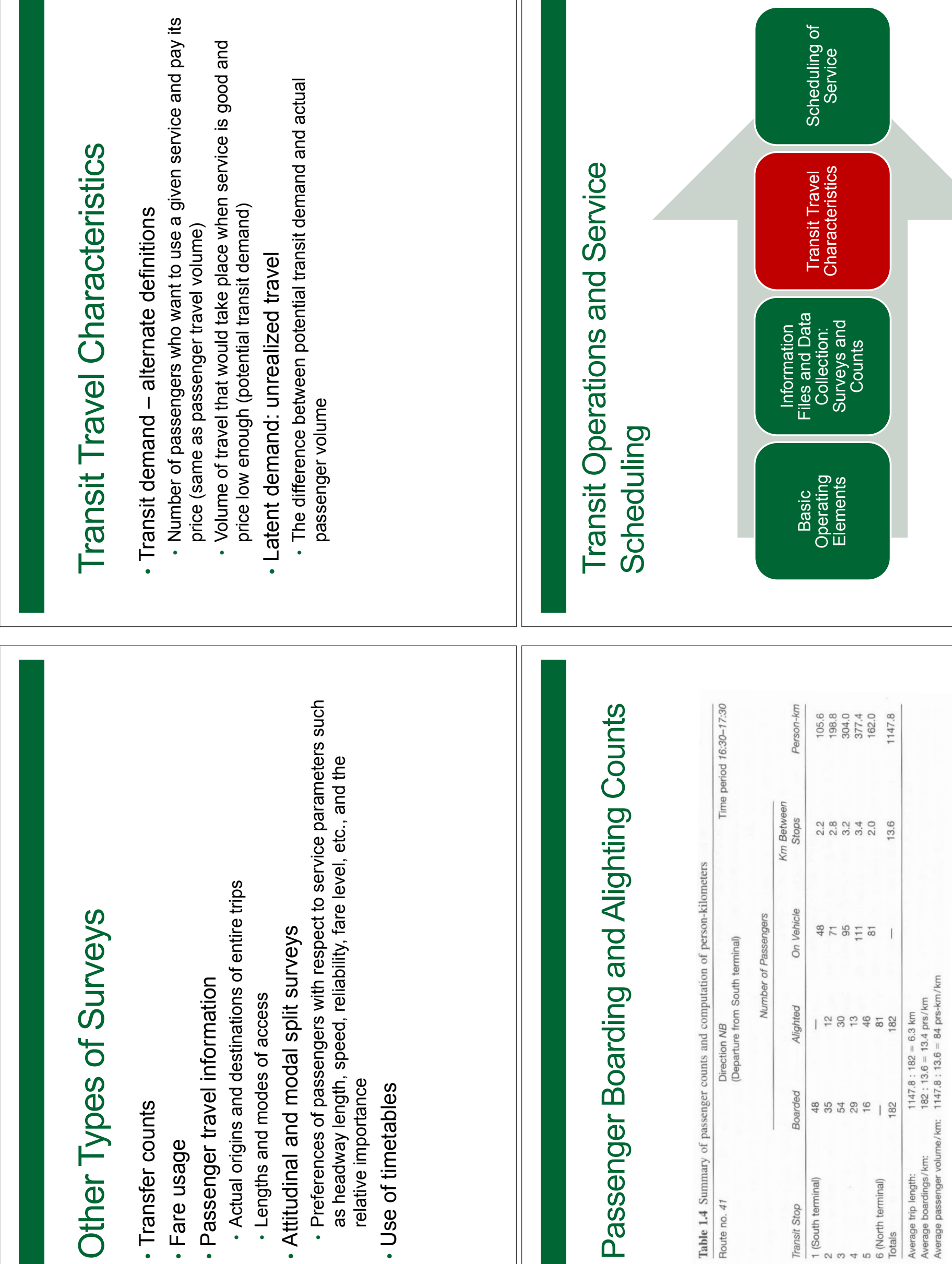

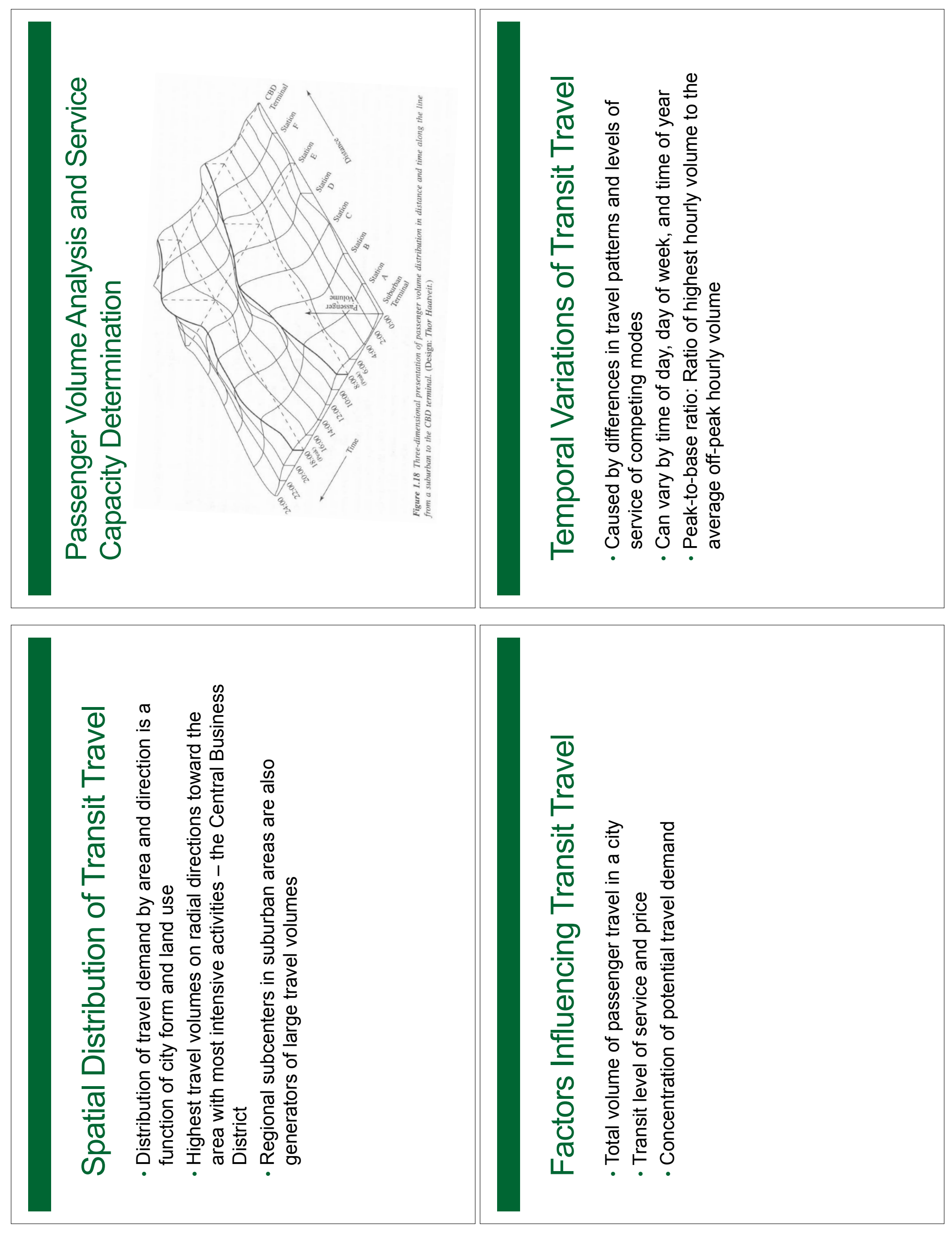

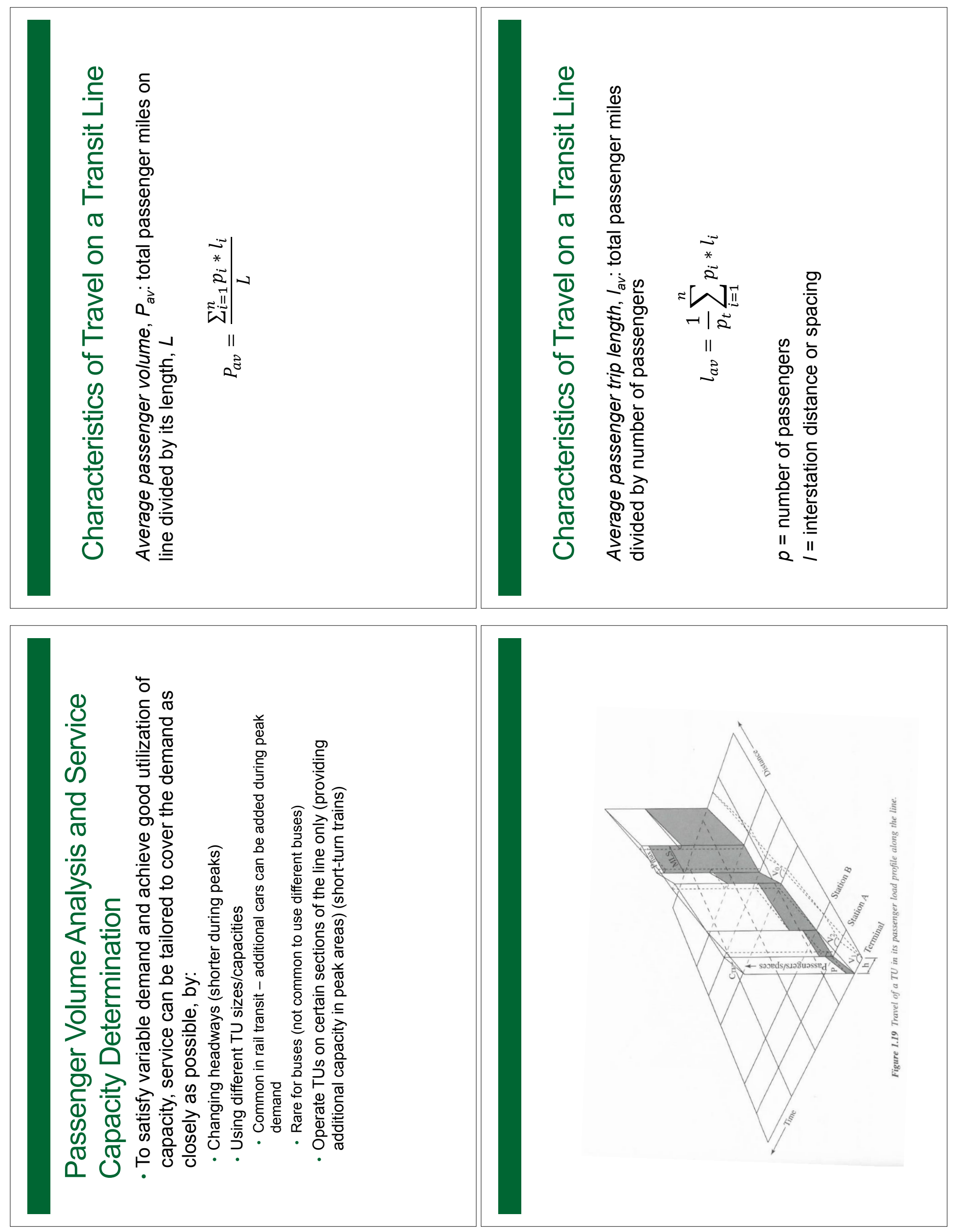

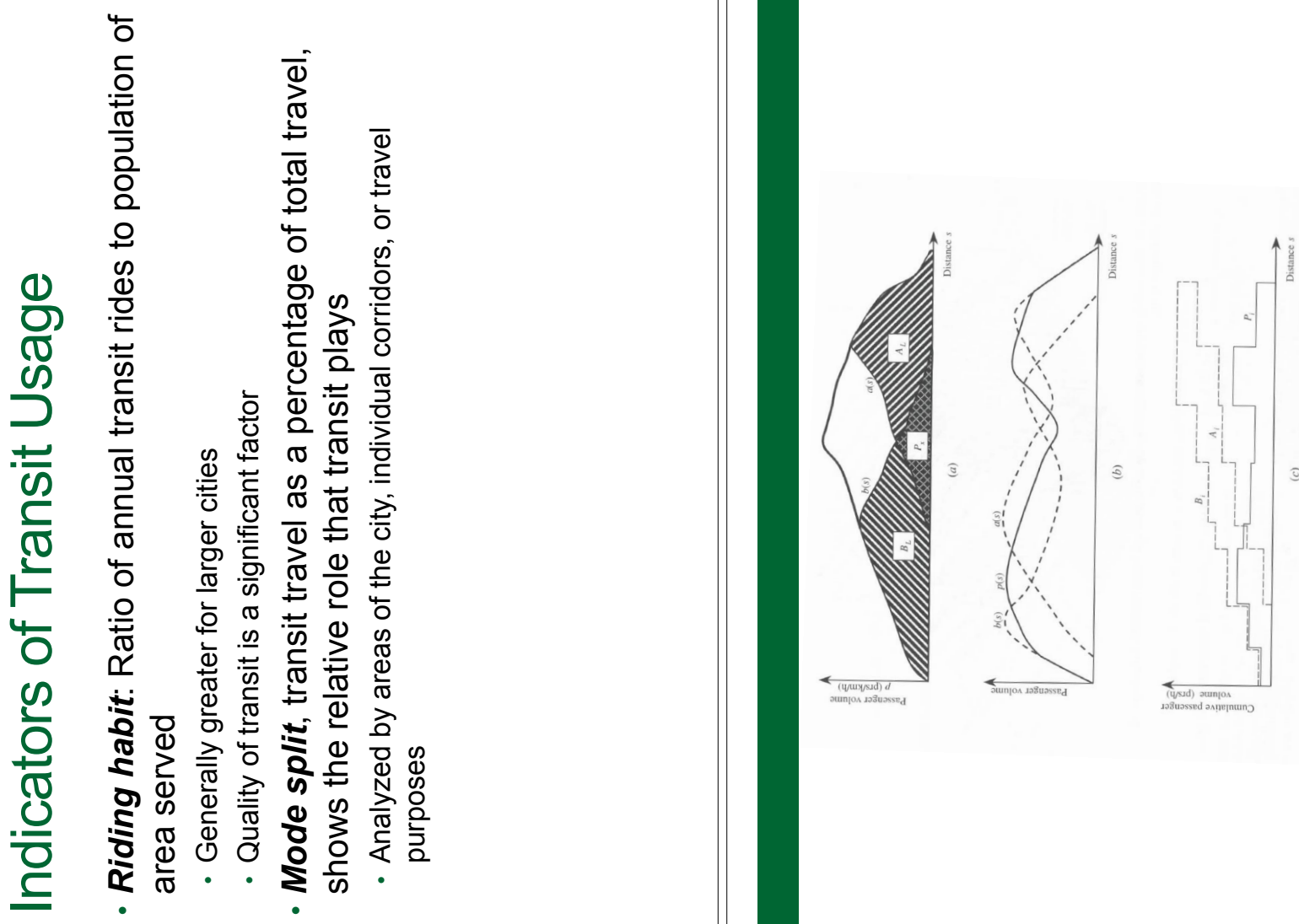

(1)

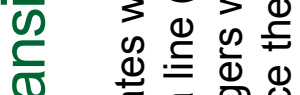

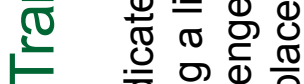

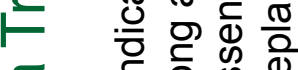

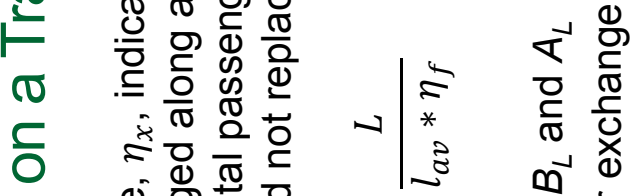

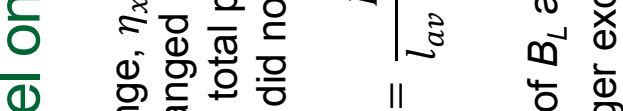

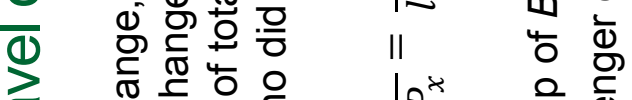

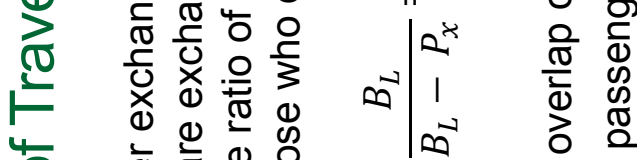

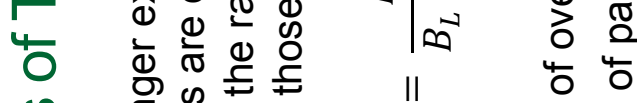

ఝ

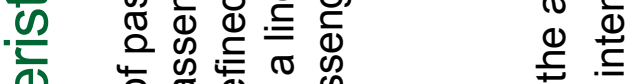

(1) पे

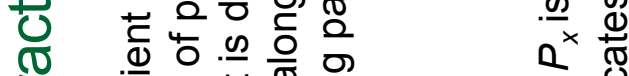

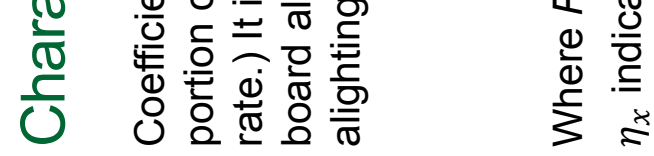

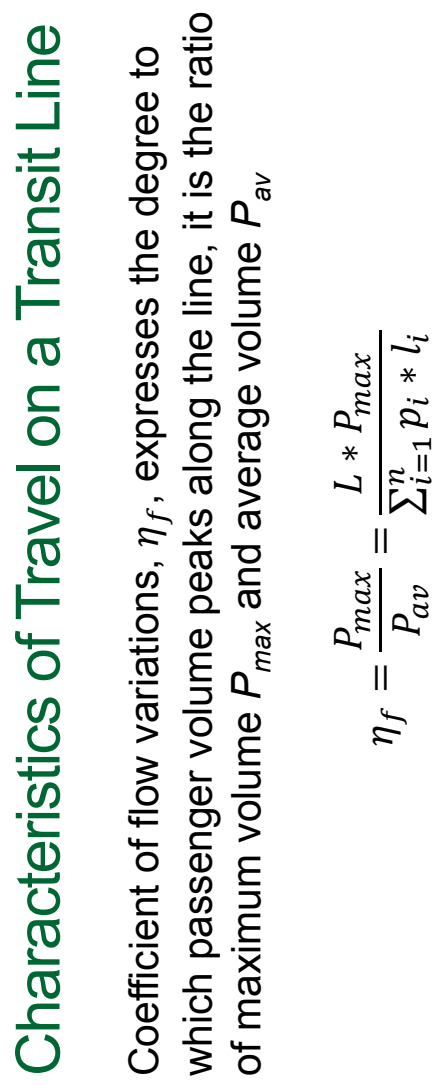



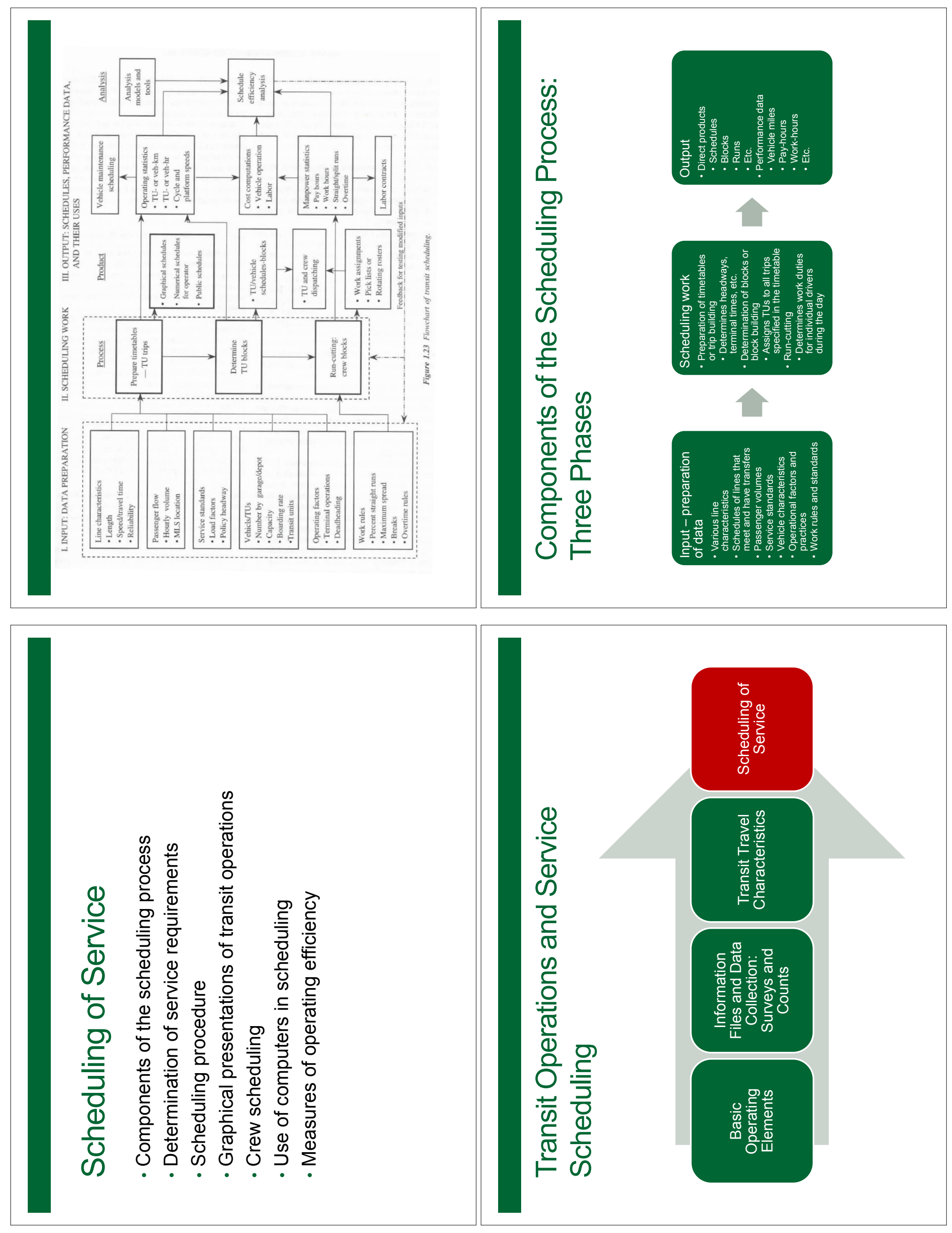

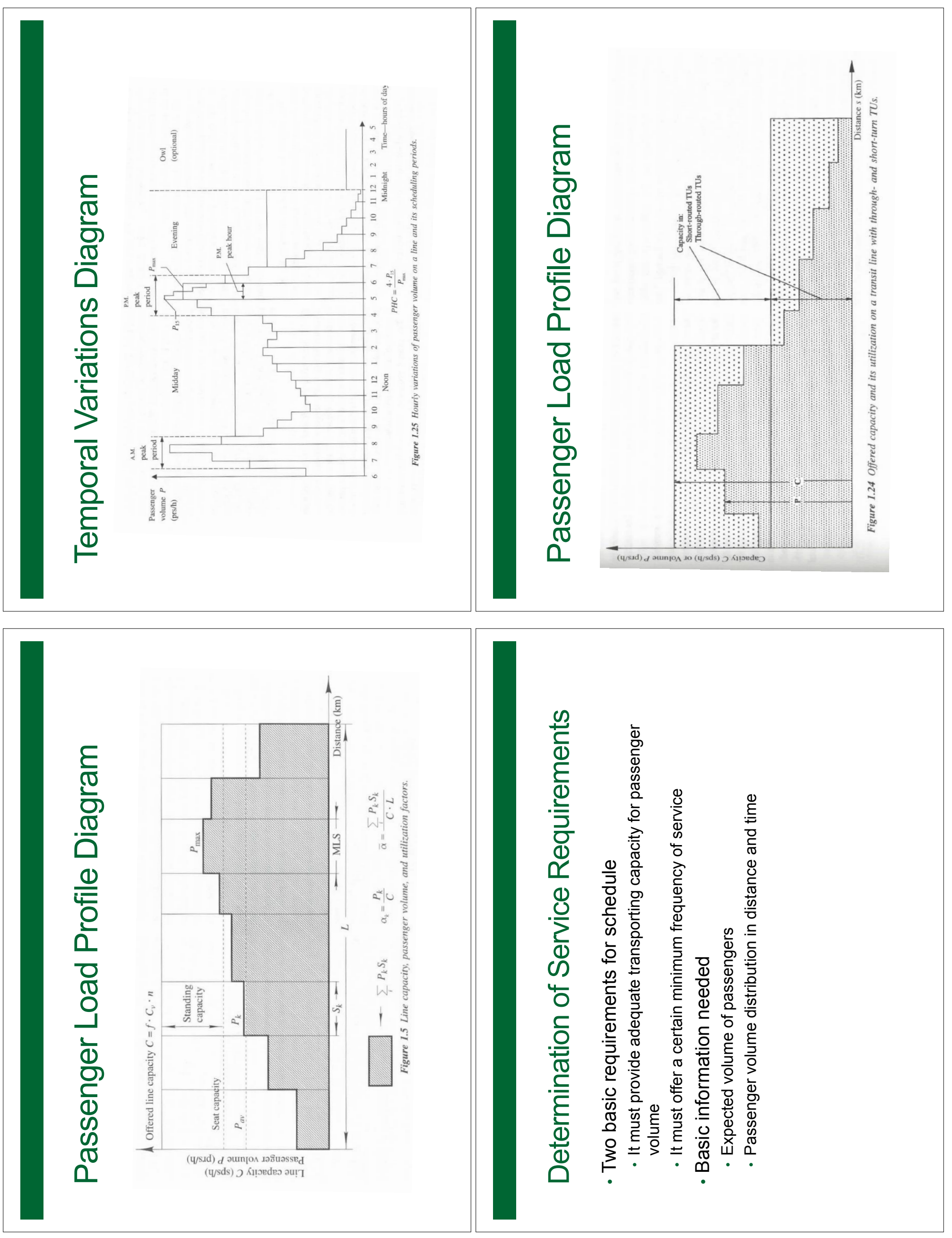

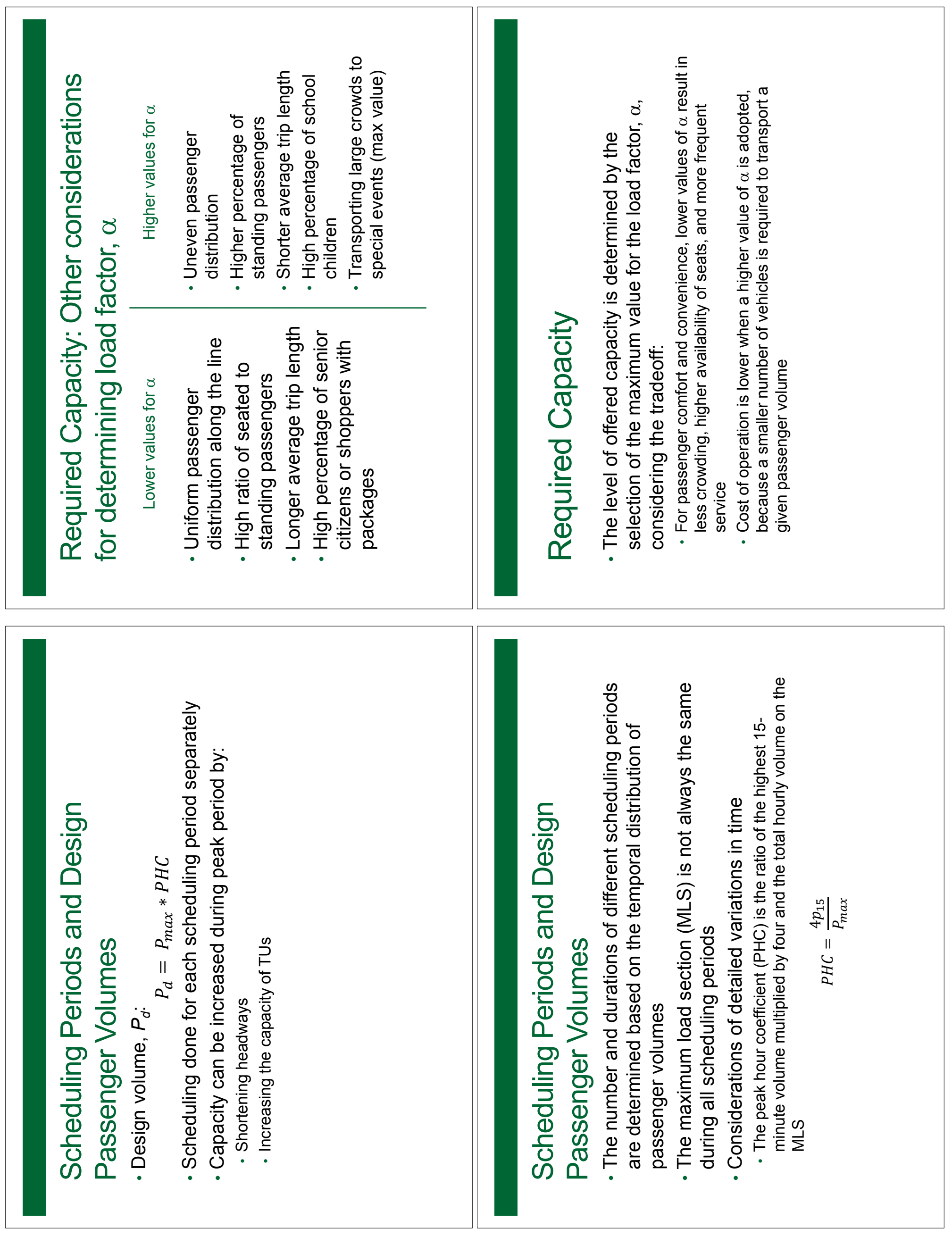

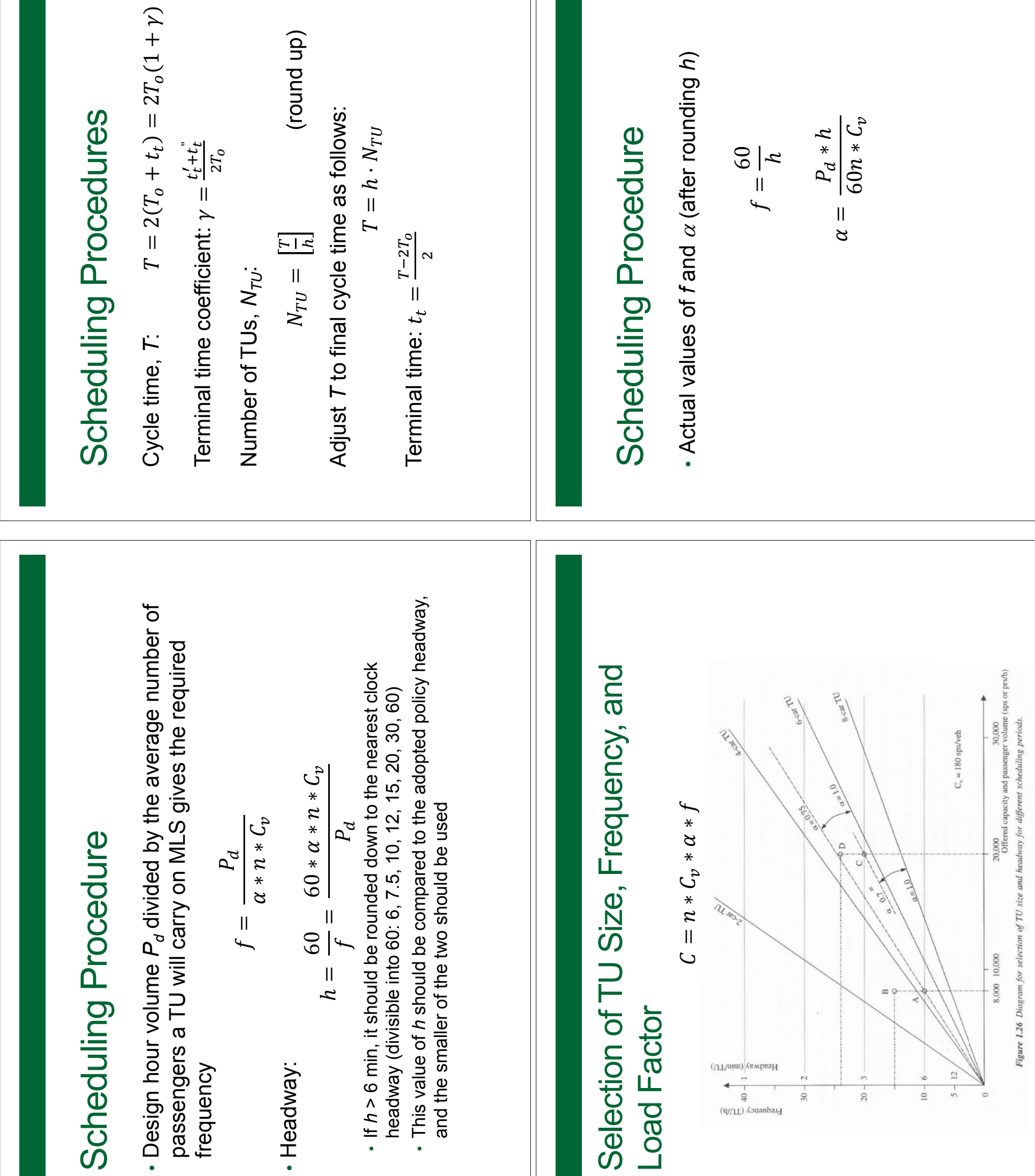

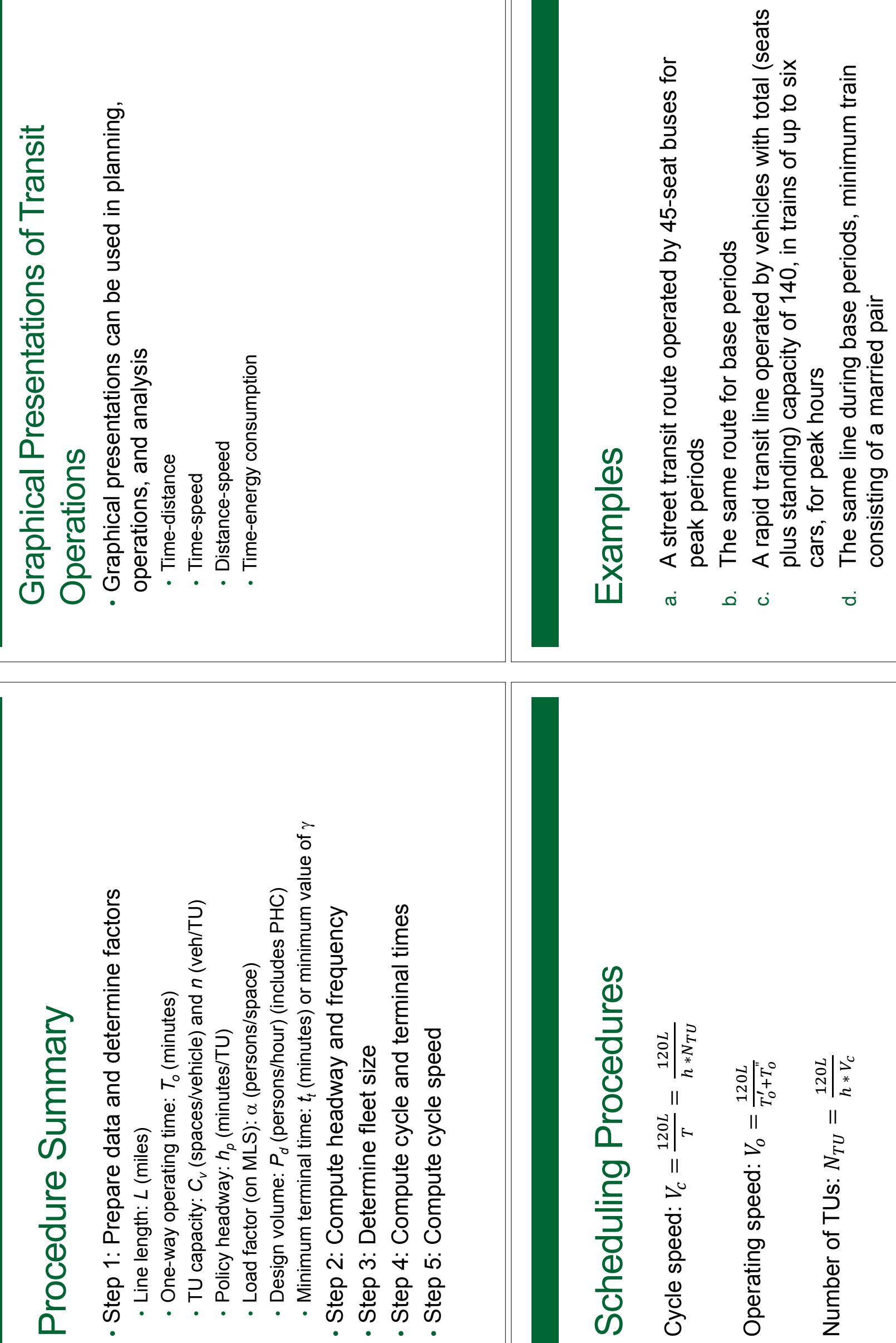

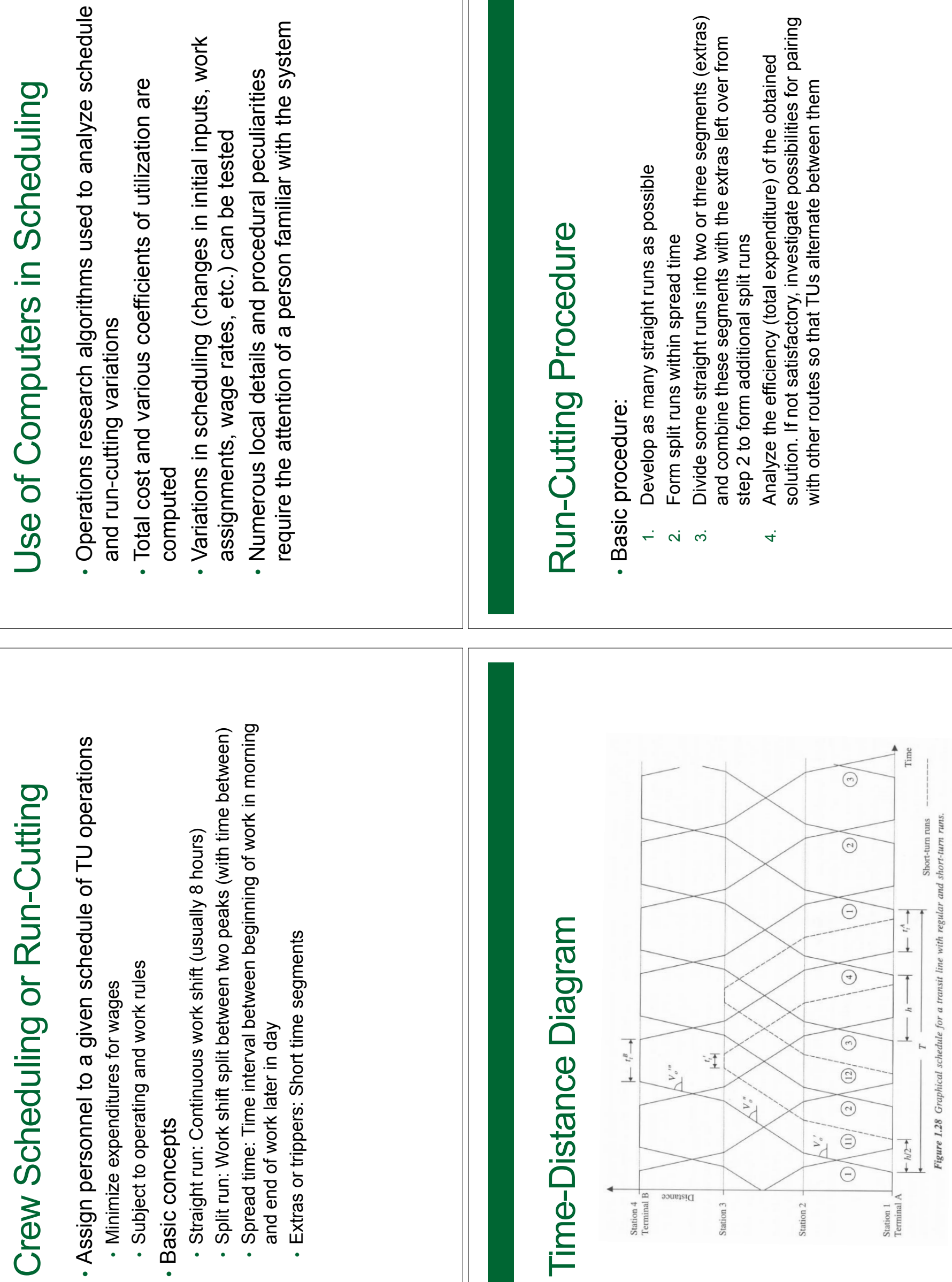


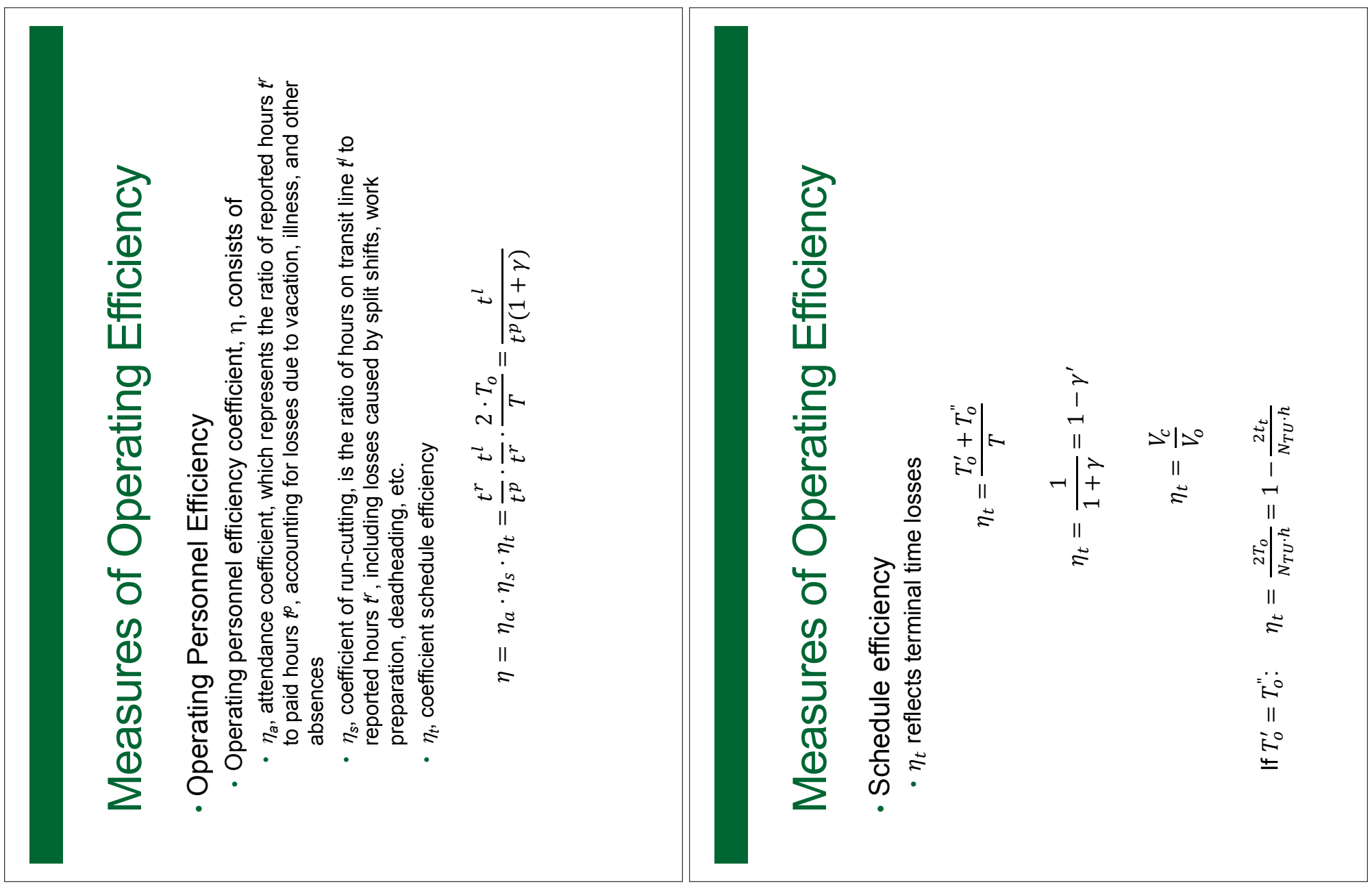




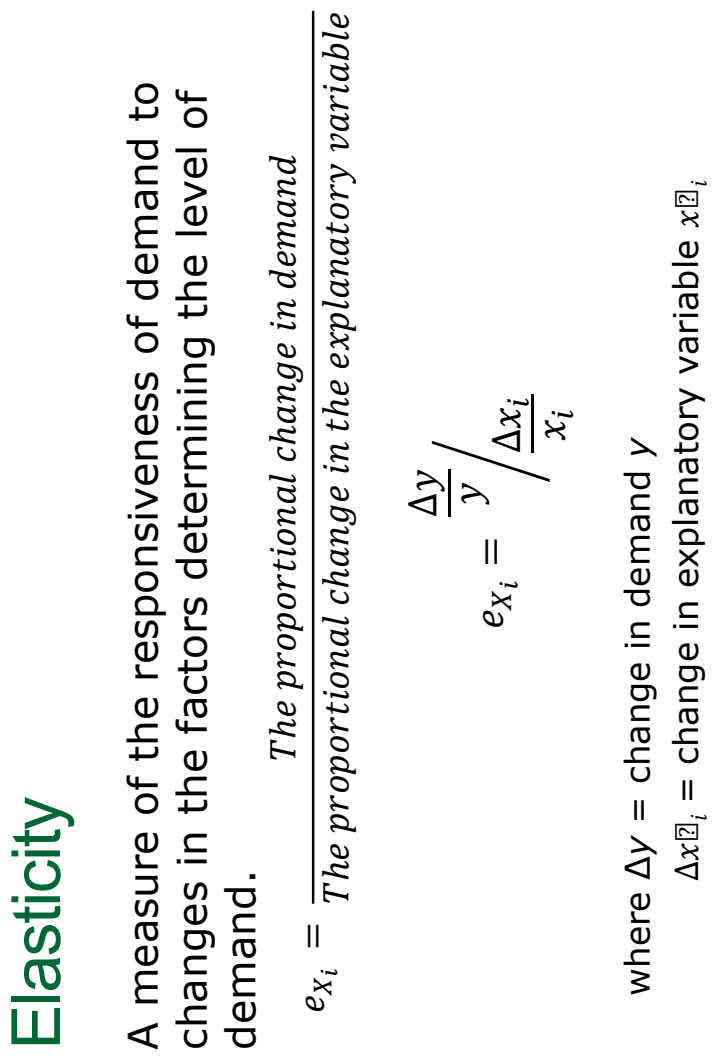

$\stackrel{0}{\frac{1}{5}}$

שֶ

음 은 는

는 을 은

ข ᄃ

F这

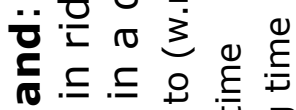

(1) OU

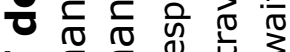

4 든 돈

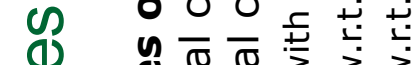

(1) y

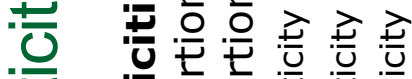

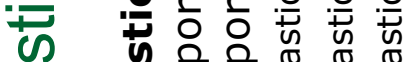

ர
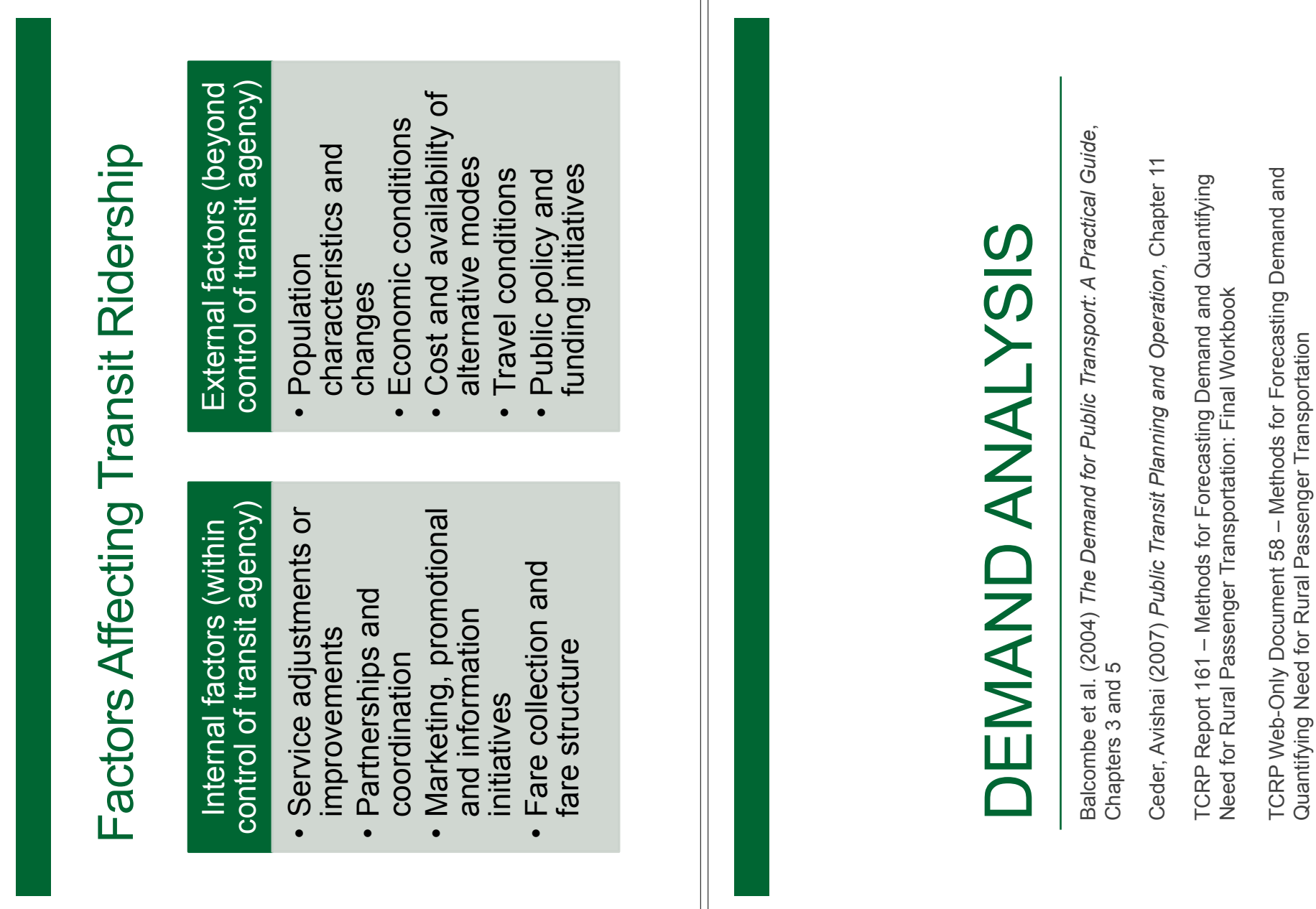

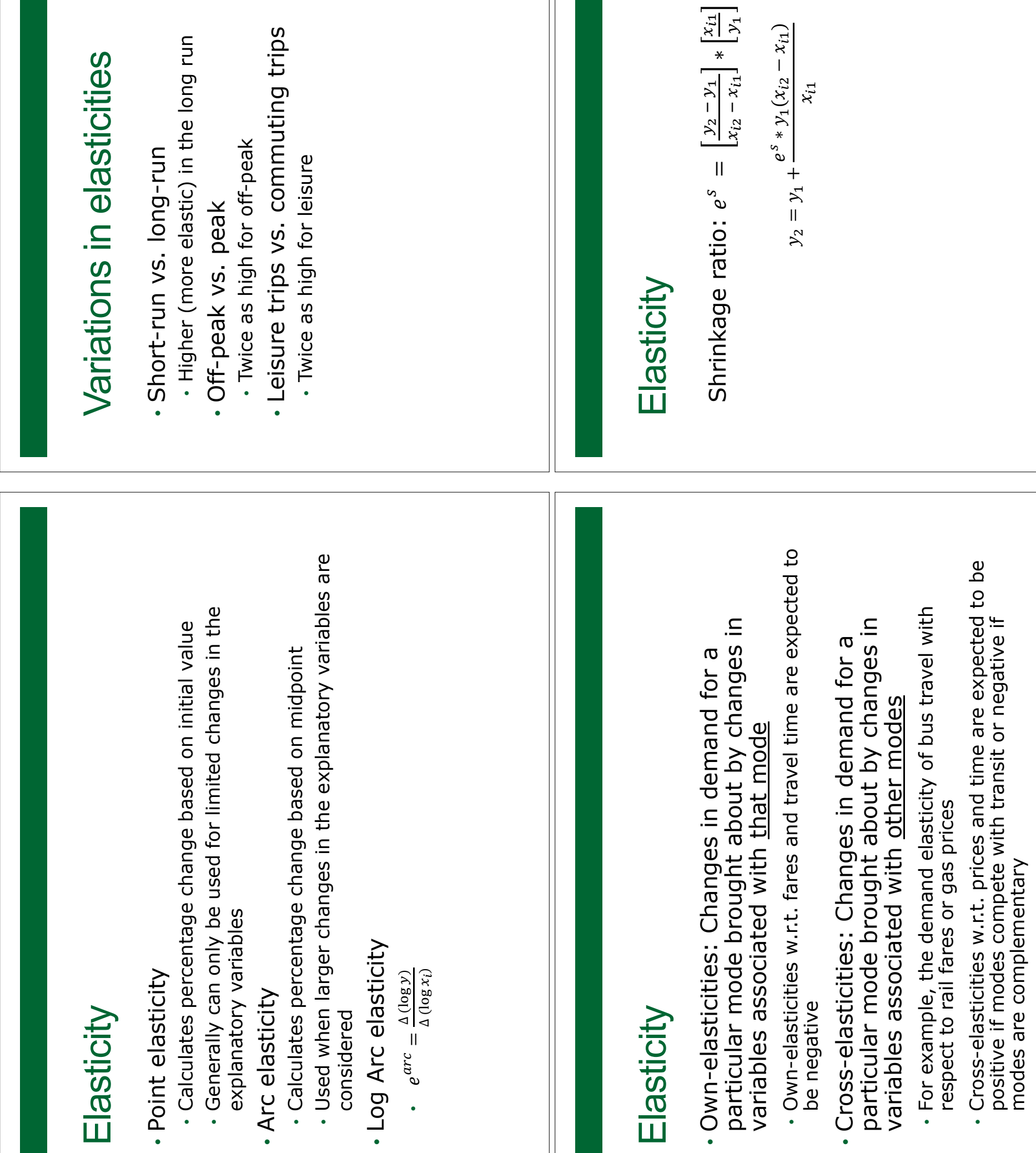

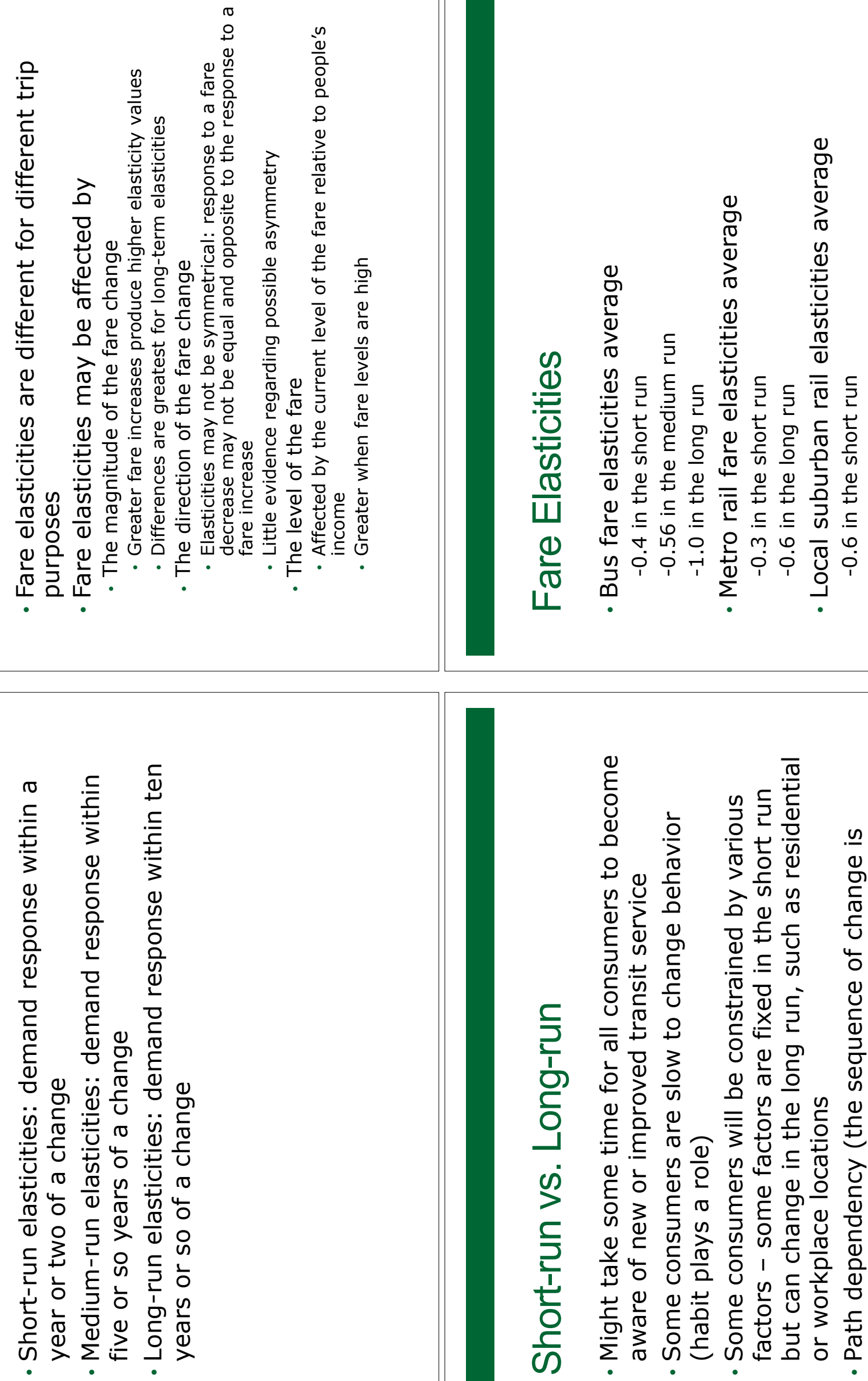

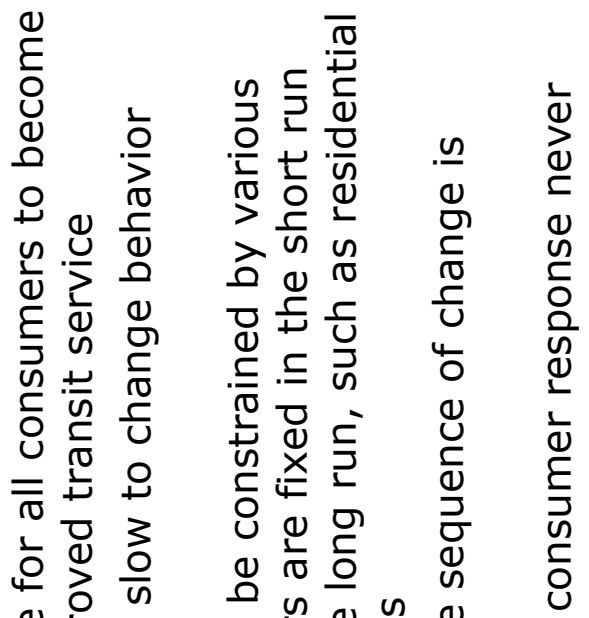

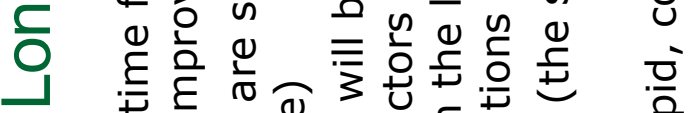

()

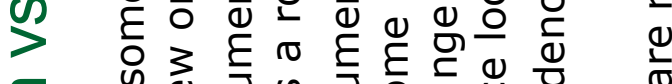

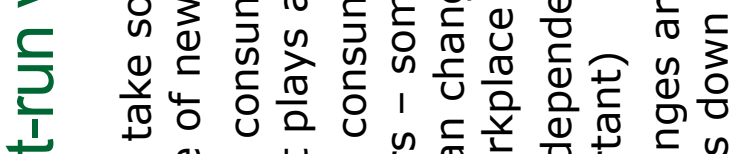

tᄂ

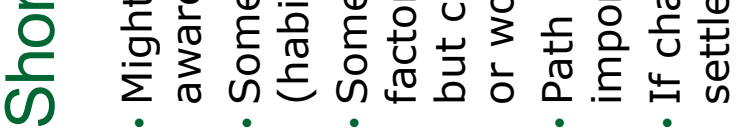



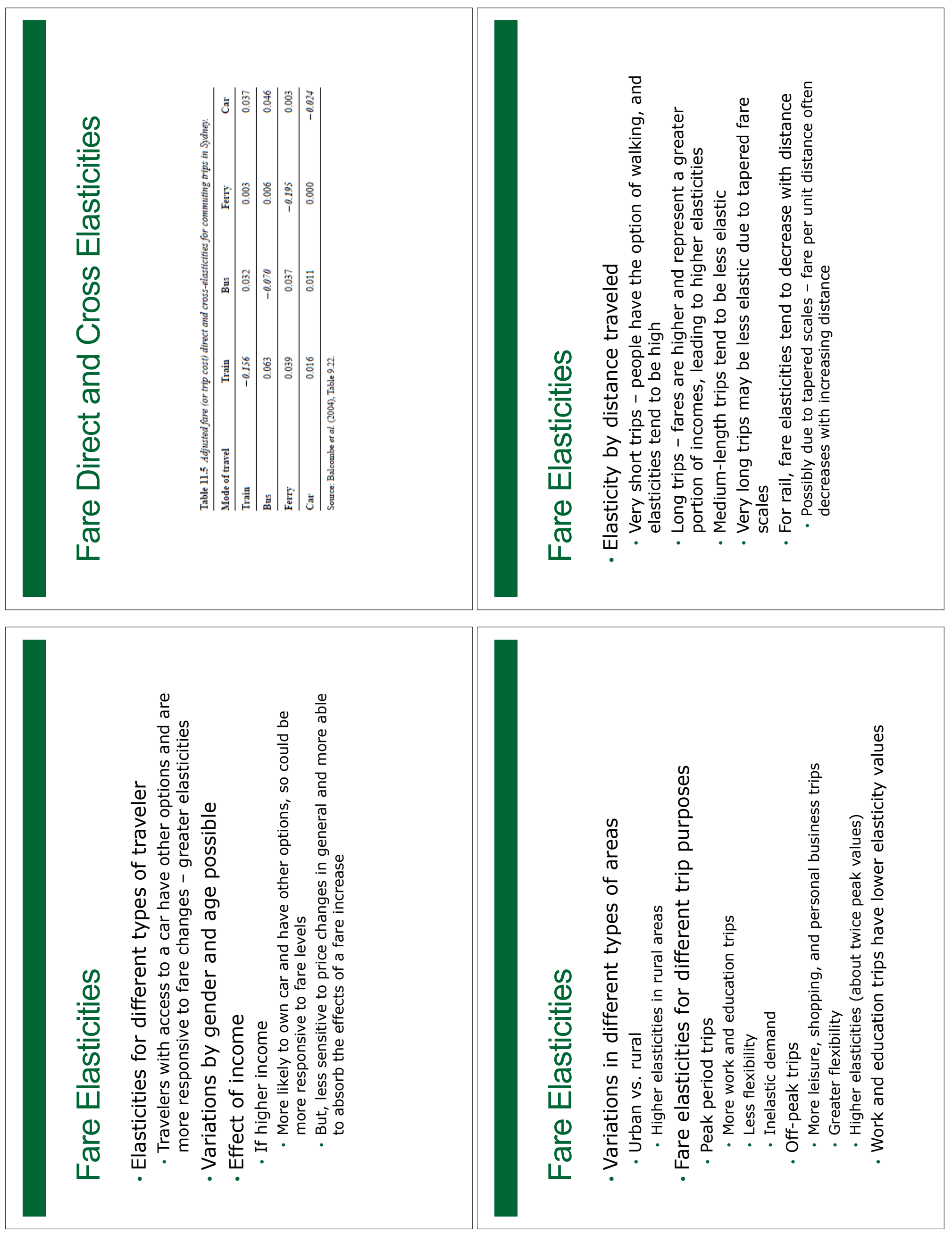


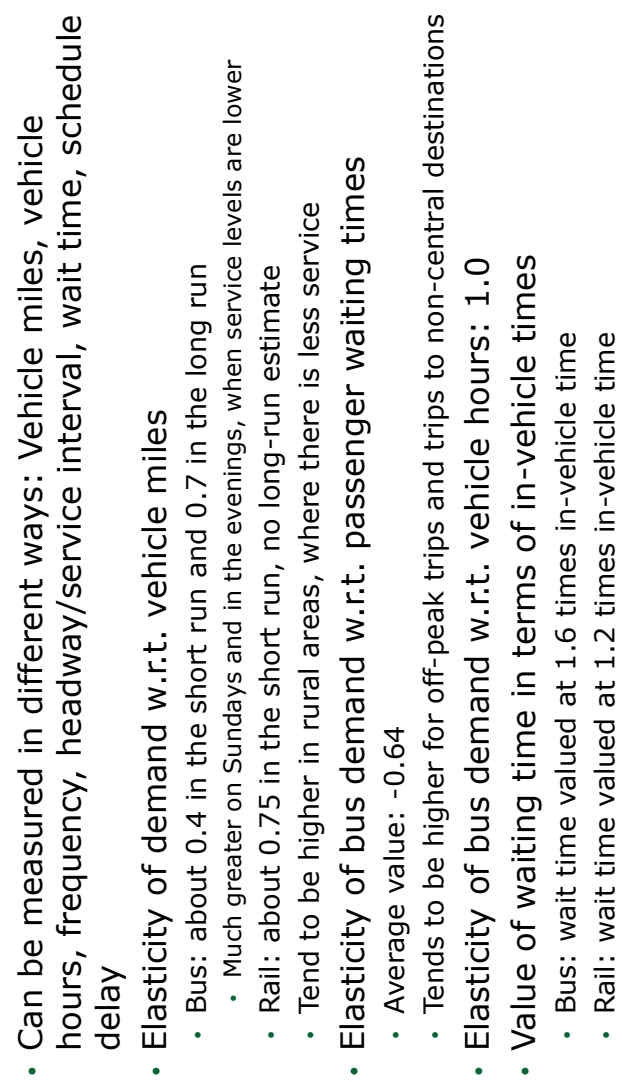

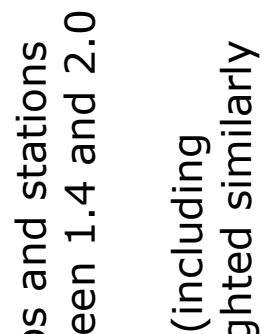

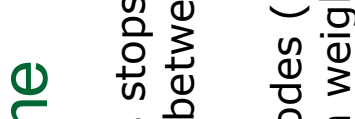

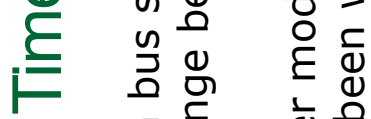

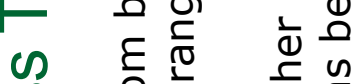

(1) 인

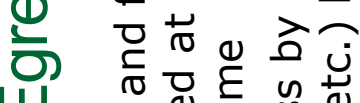

山

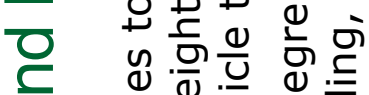

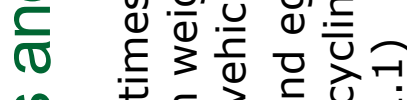

( ) $>\frac{5}{\sigma}$ U

(1) ㄸำ

U)

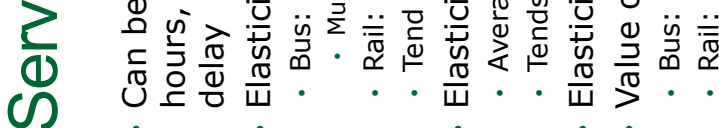

ব

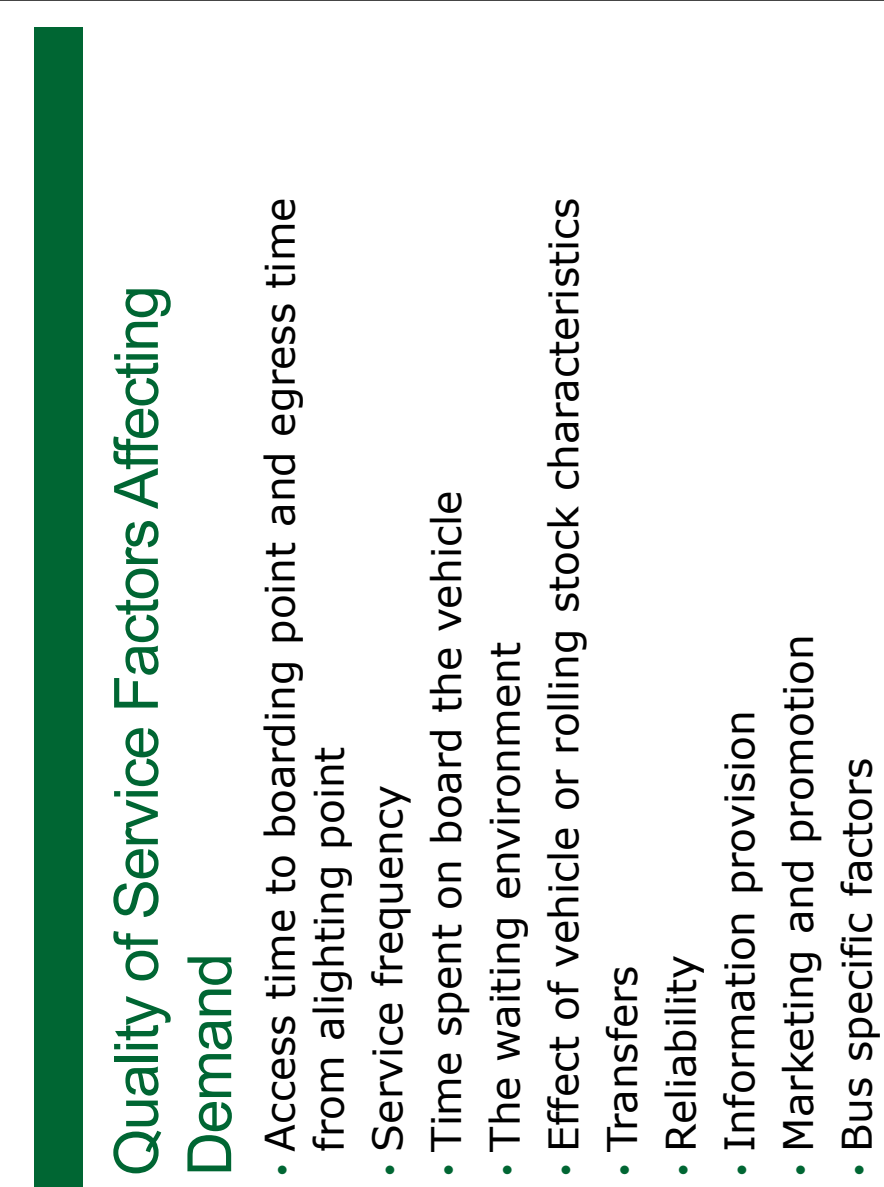

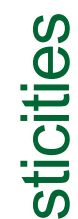

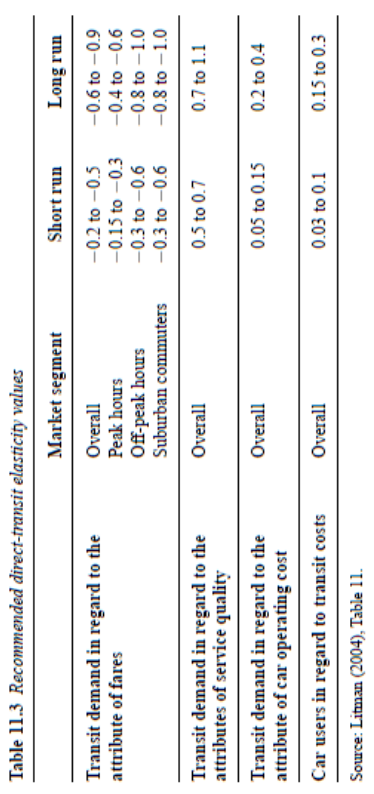

ธี 

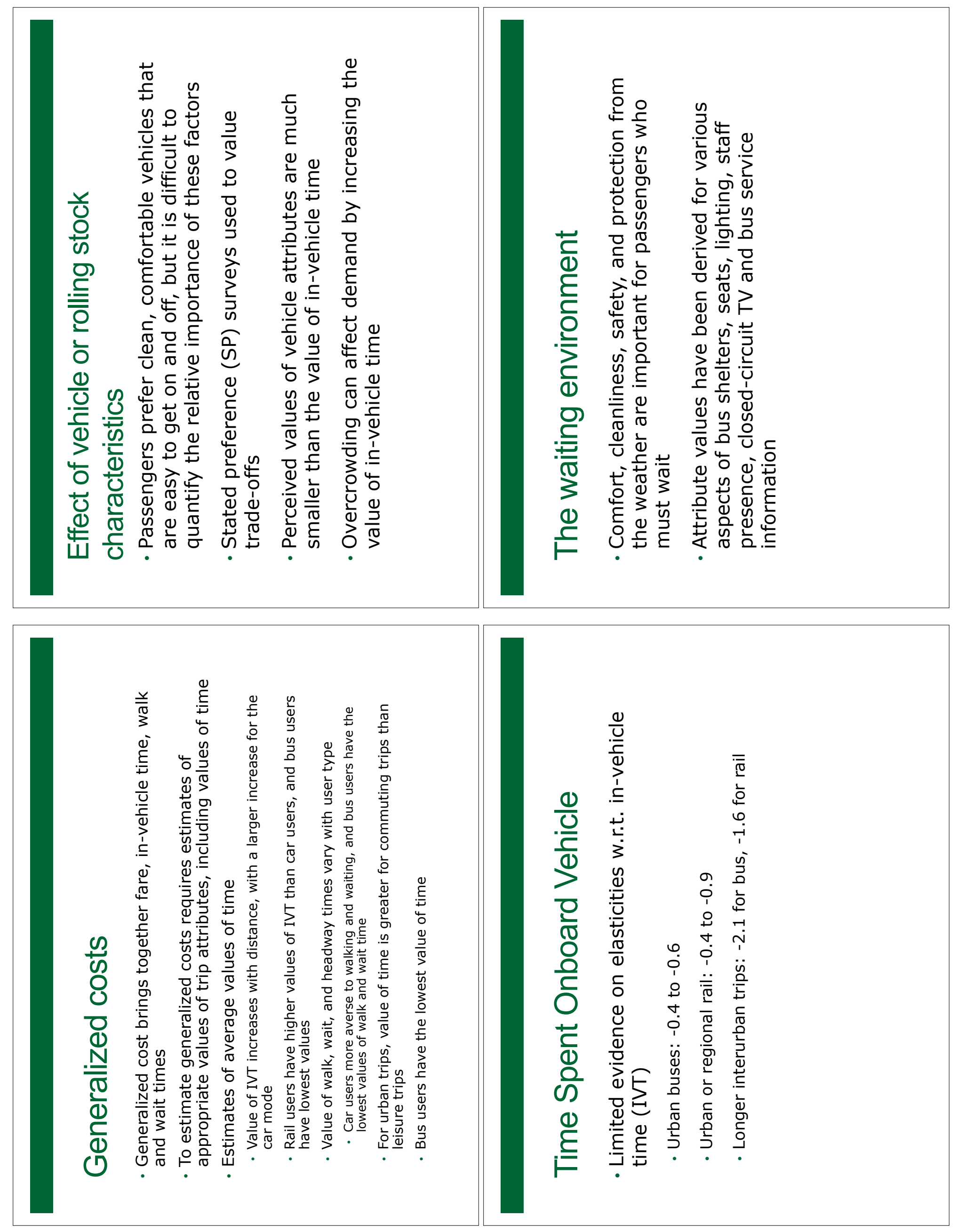

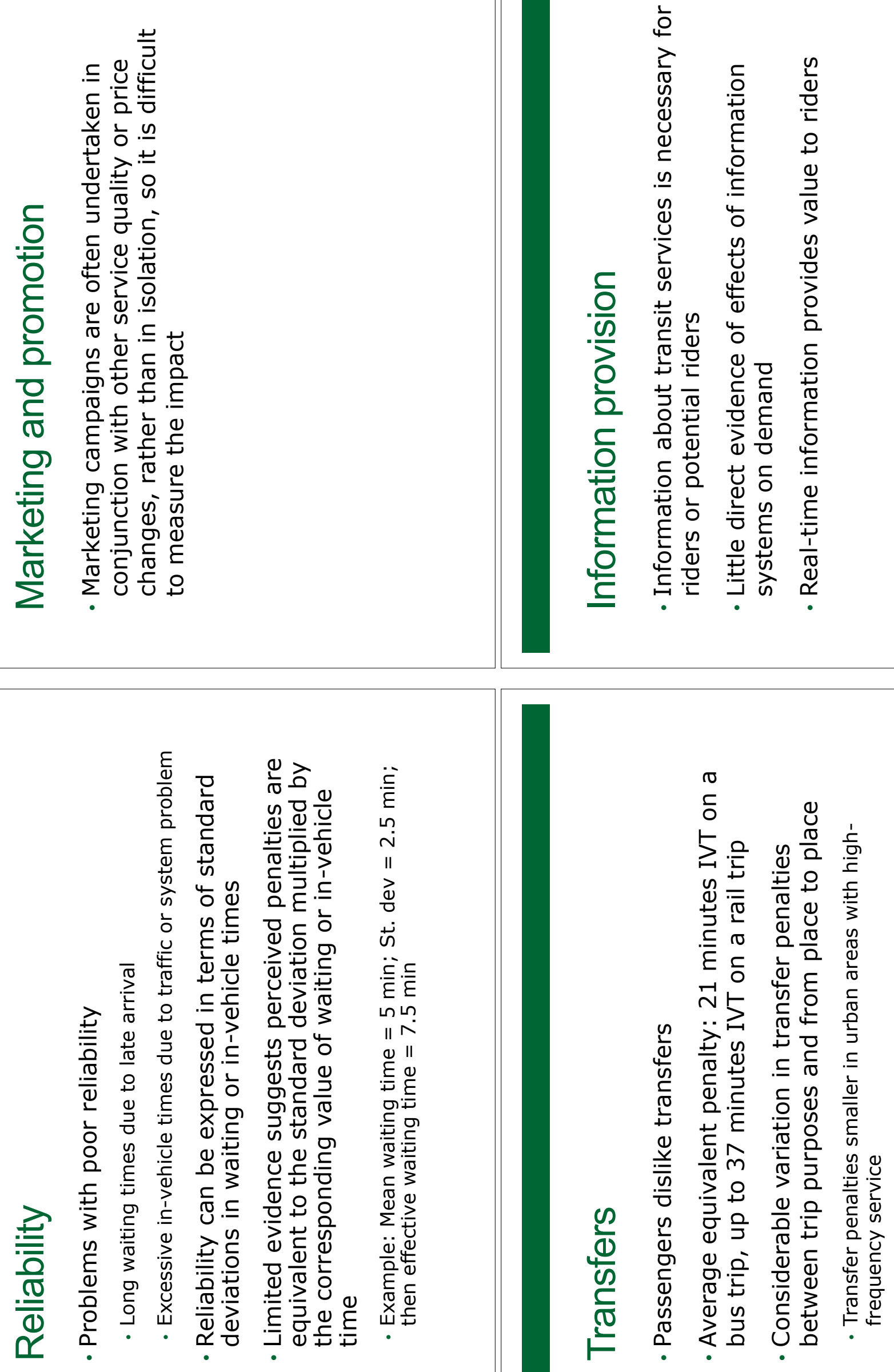

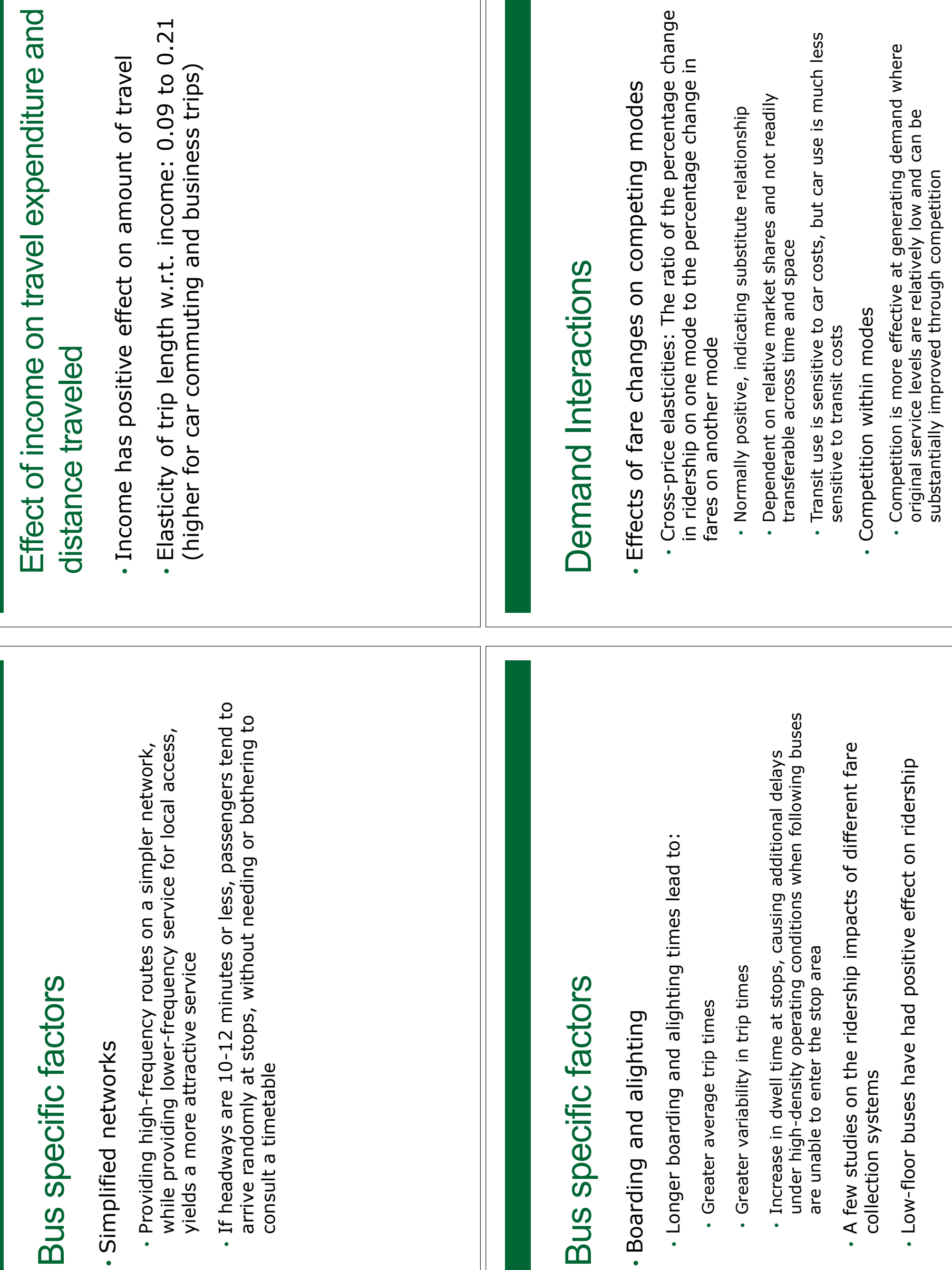

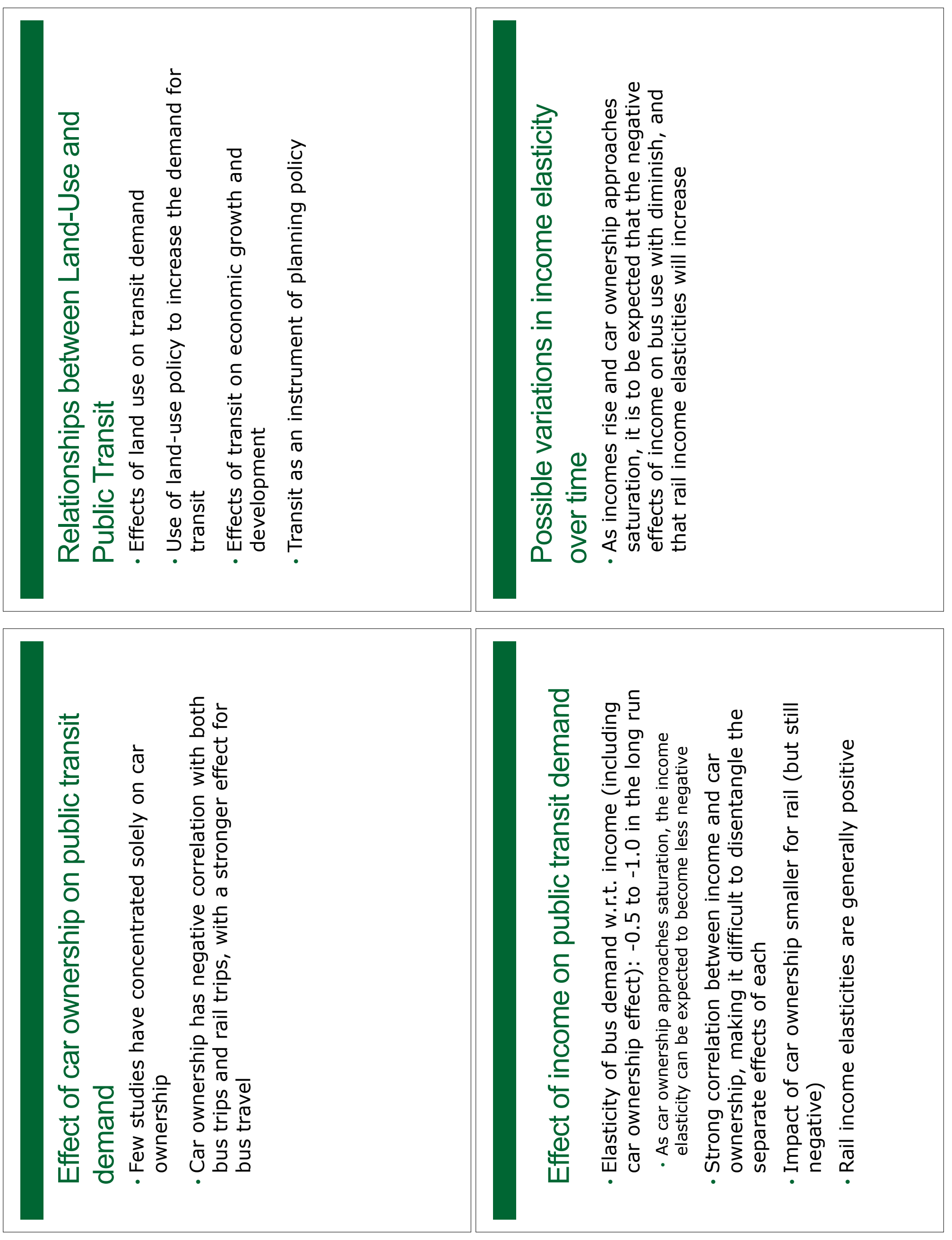

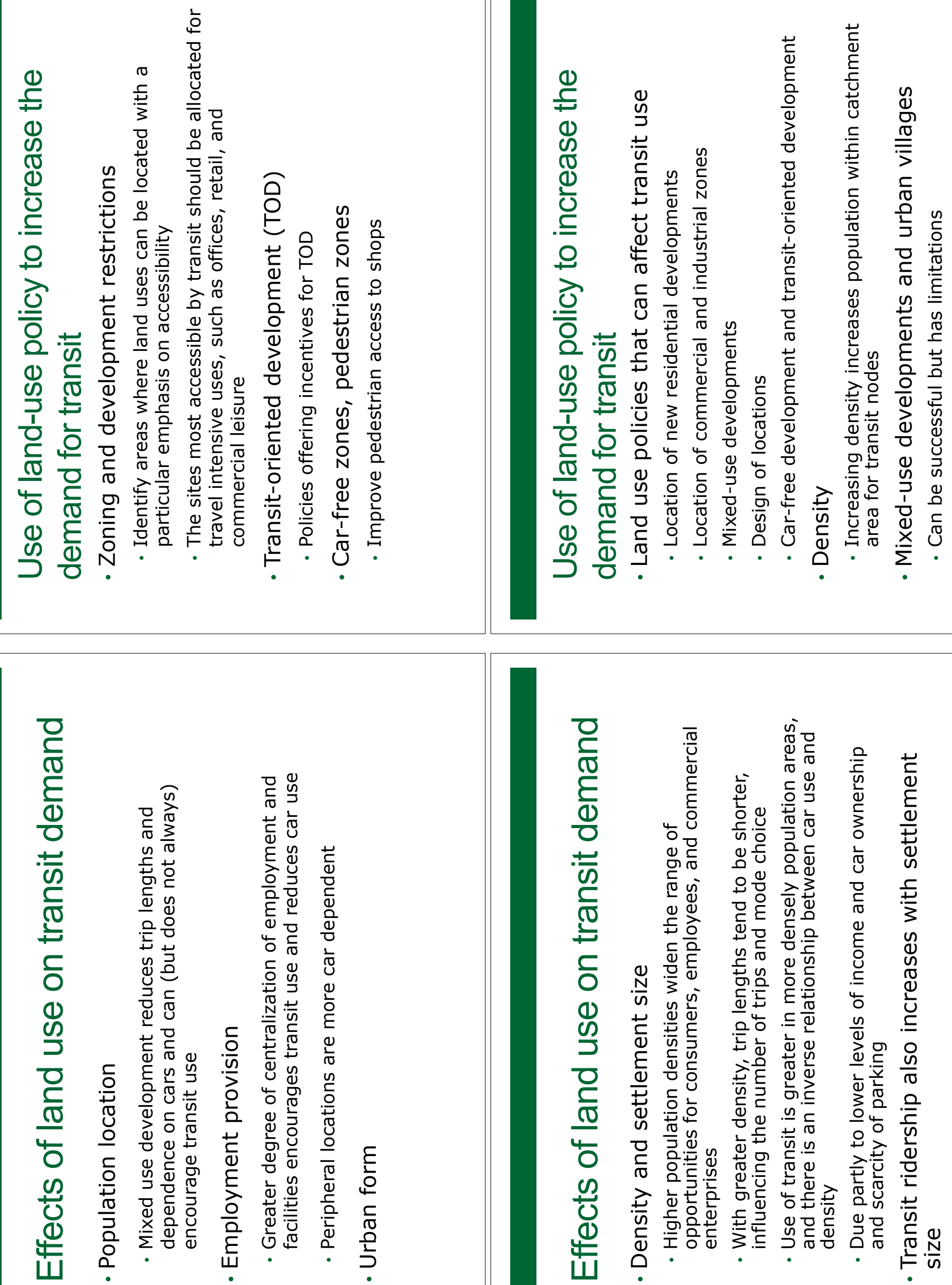
$\frac{\mathscr{d}}{\frac{0}{\bar{c}}}$

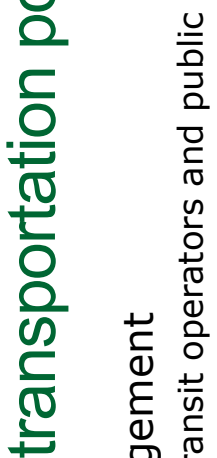

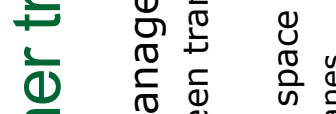

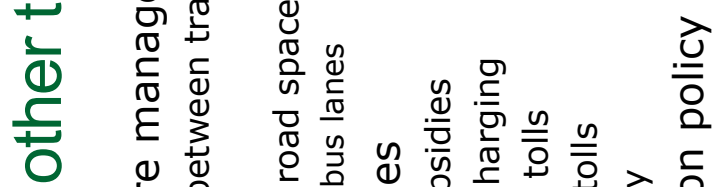

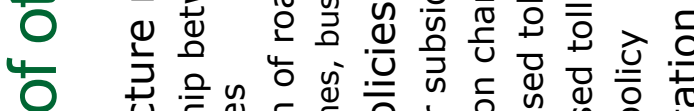

() 곤

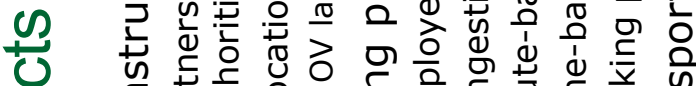

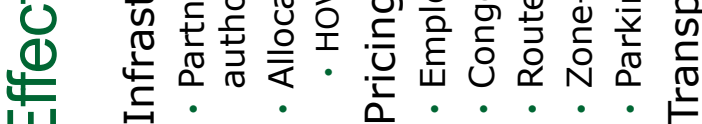

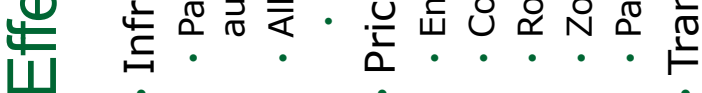

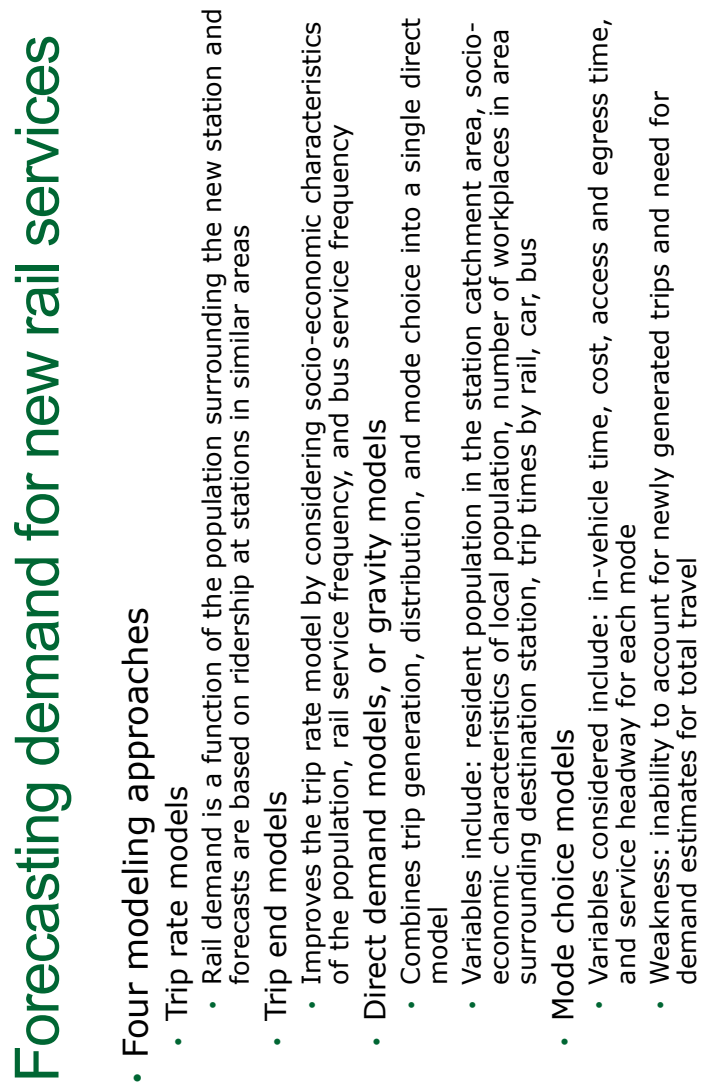

$\frac{d}{0}$

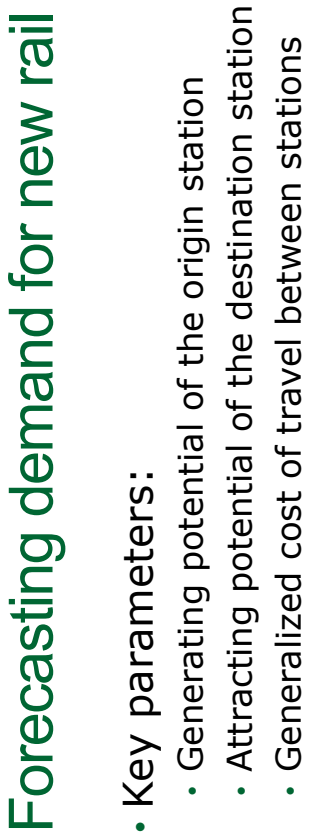

$\stackrel{\frac{0}{2}}{\frac{1}{2}}$

రั)

$\subseteq$

음
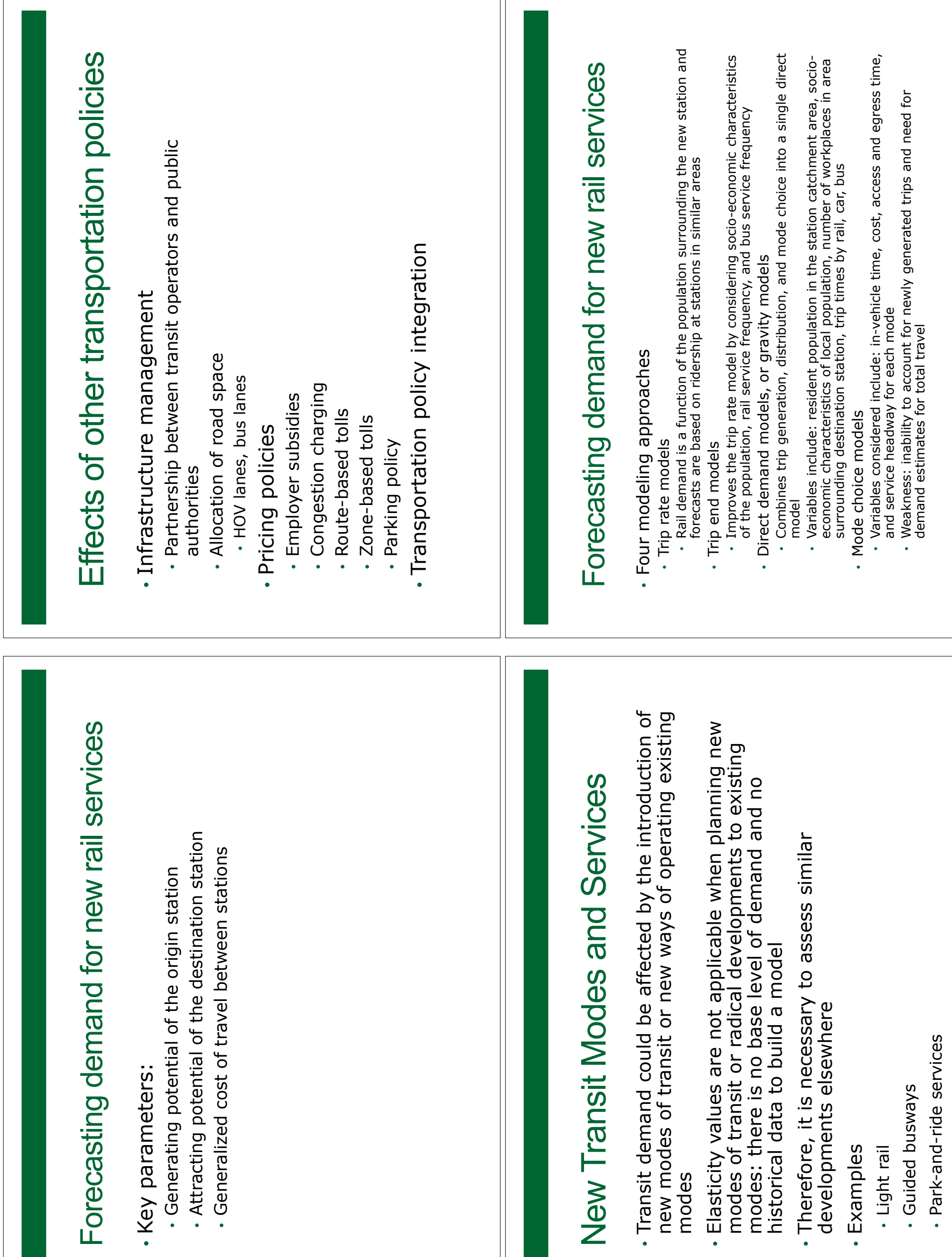

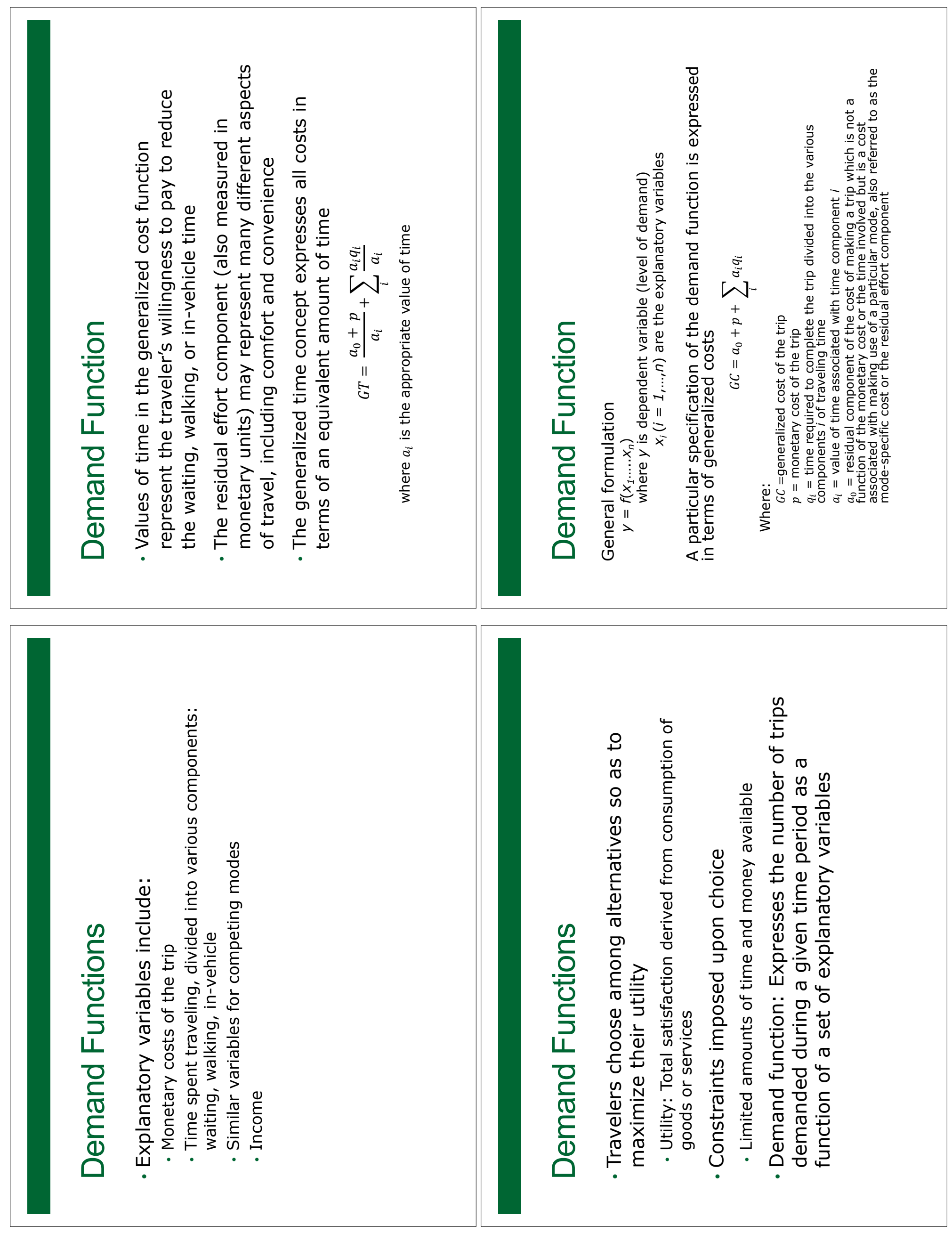


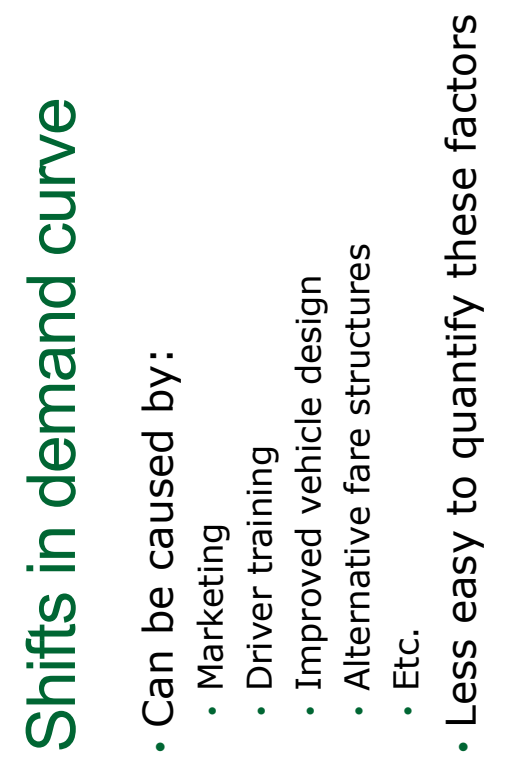

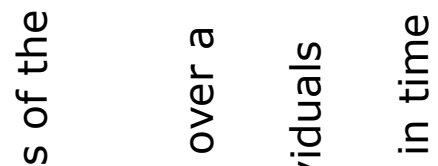

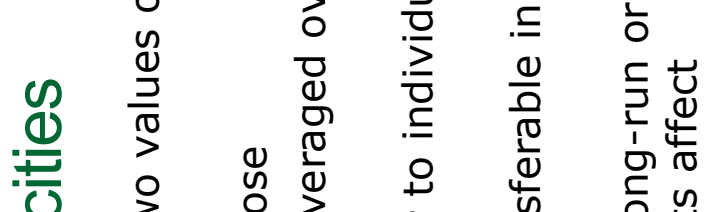

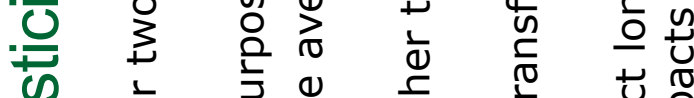

ฮั

(1)

O

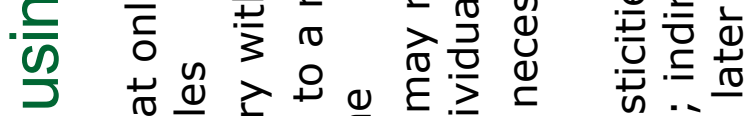

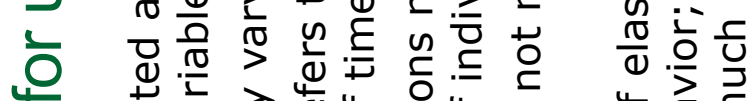

(

क उ)

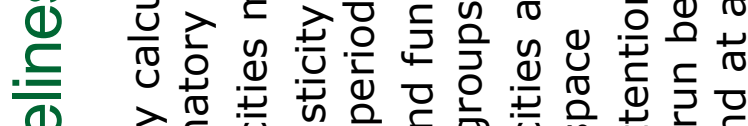

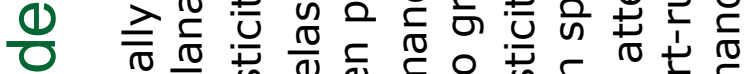

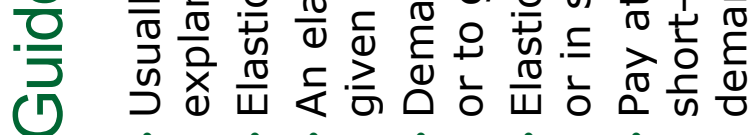
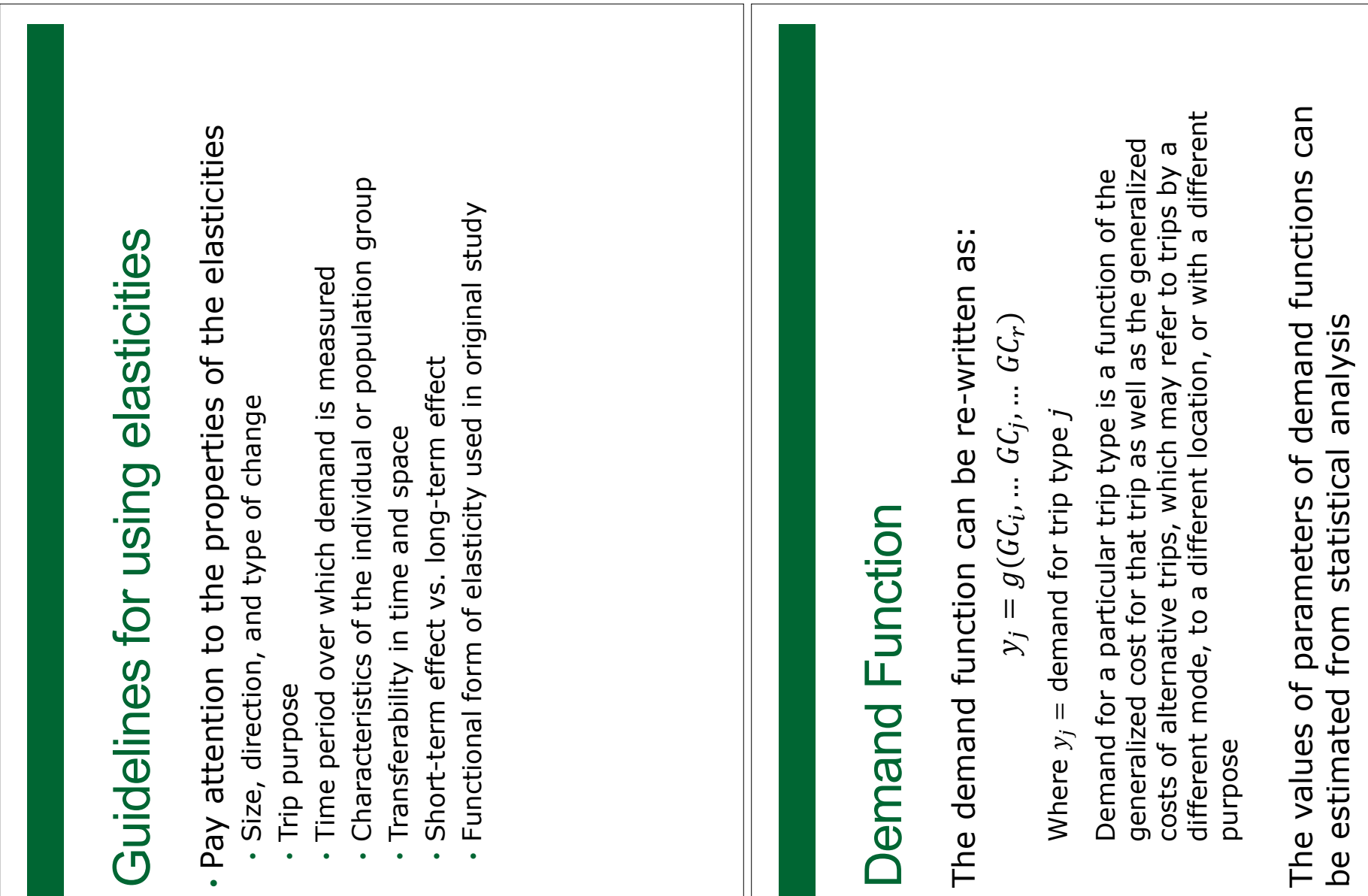

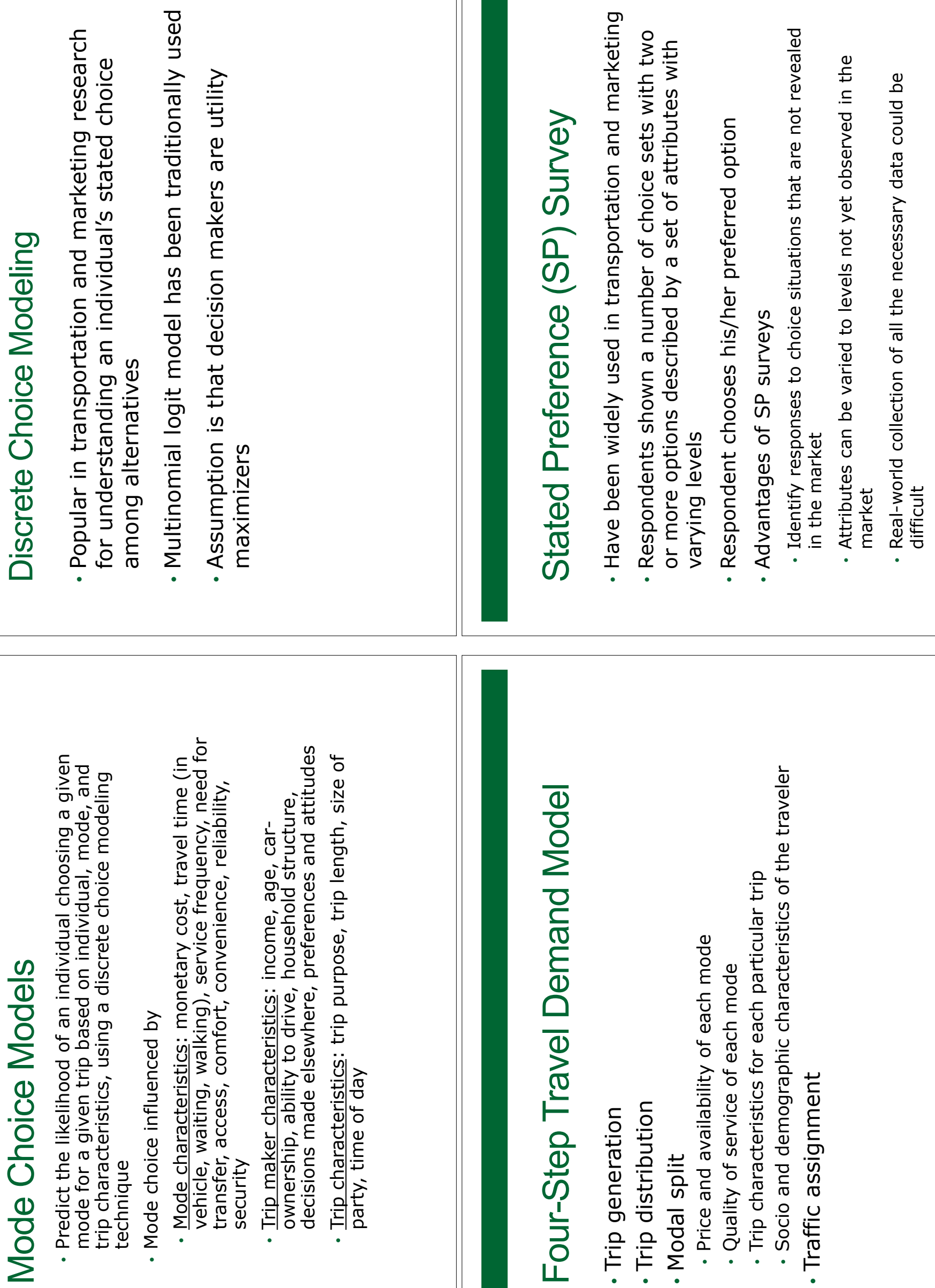

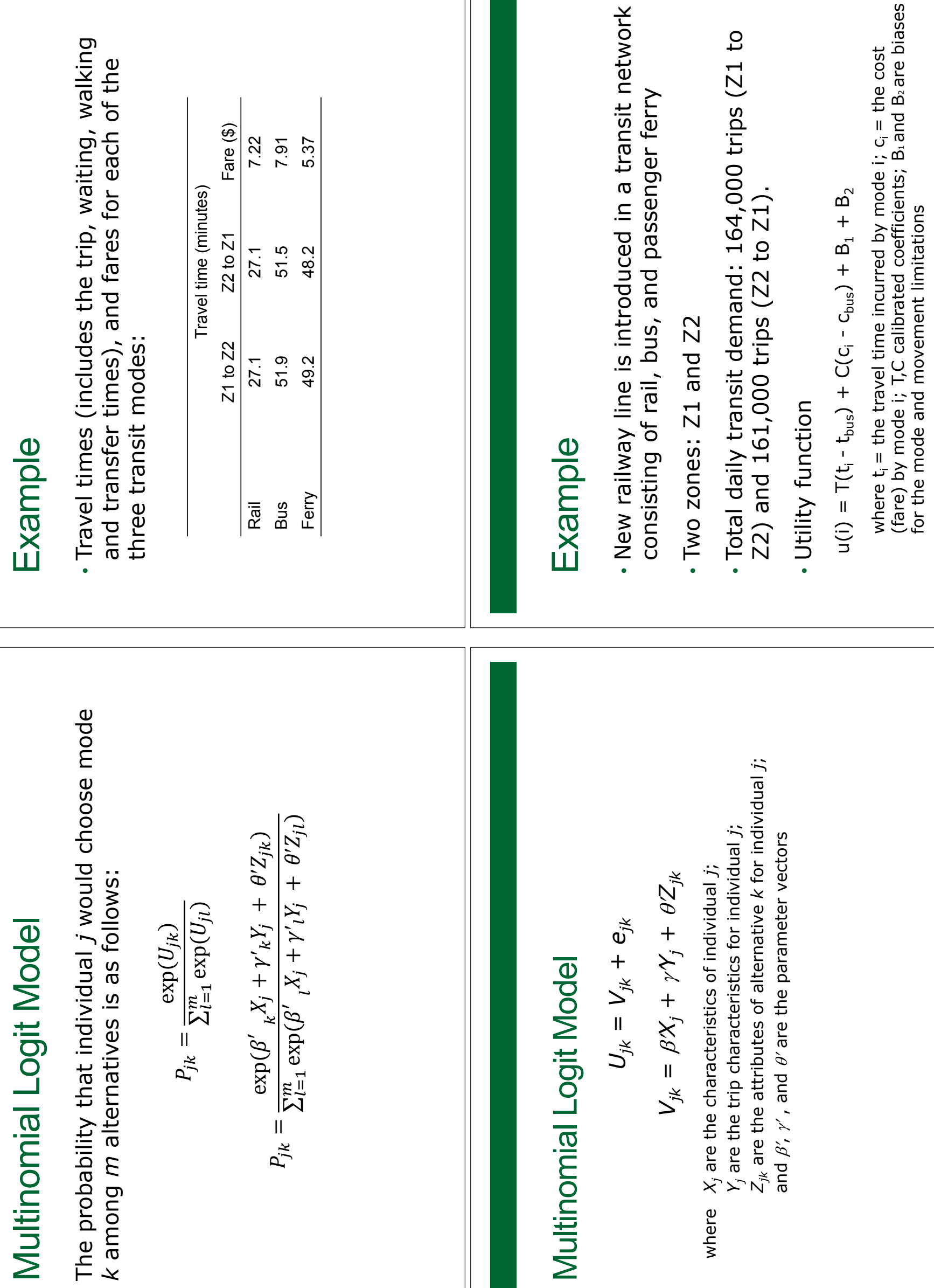

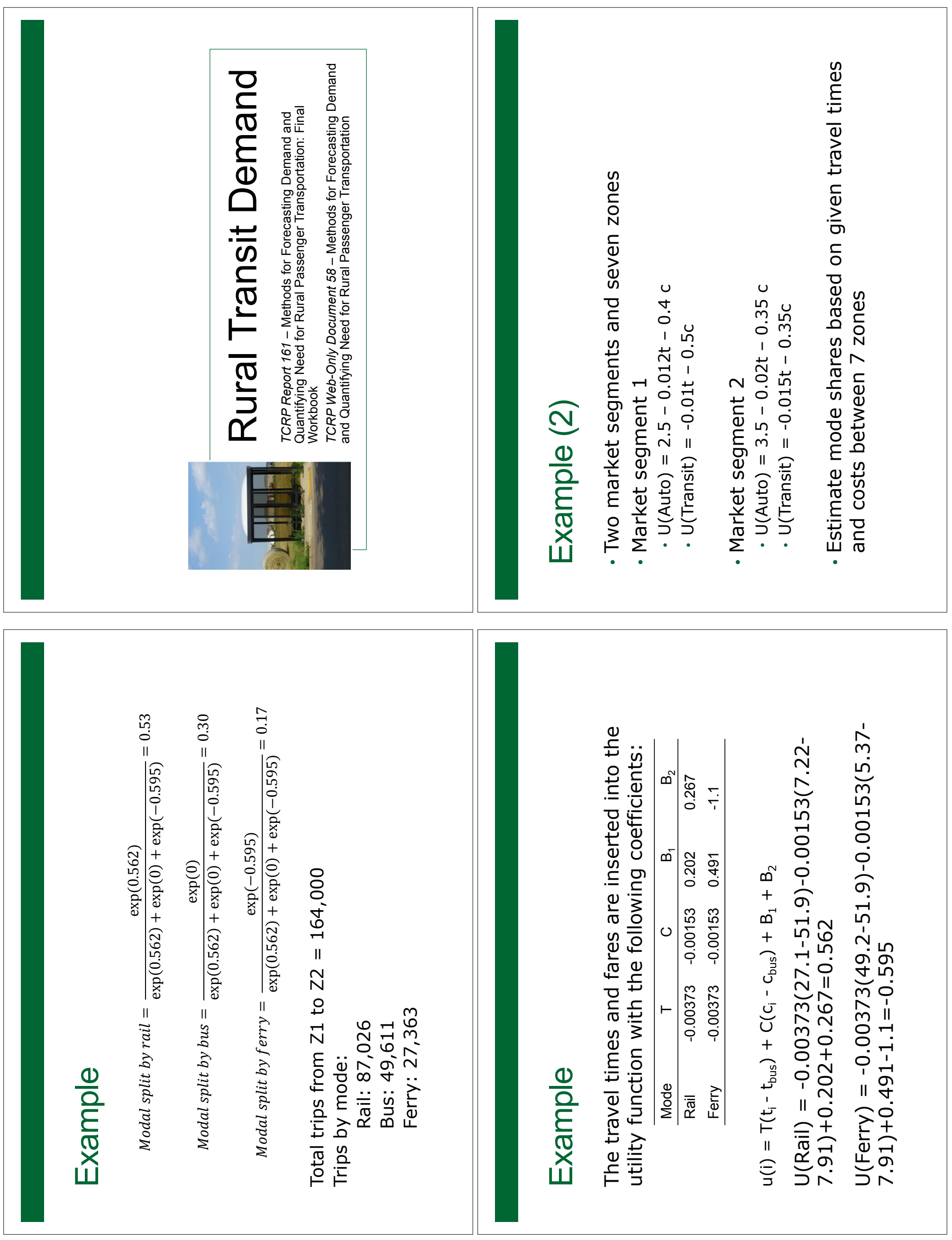

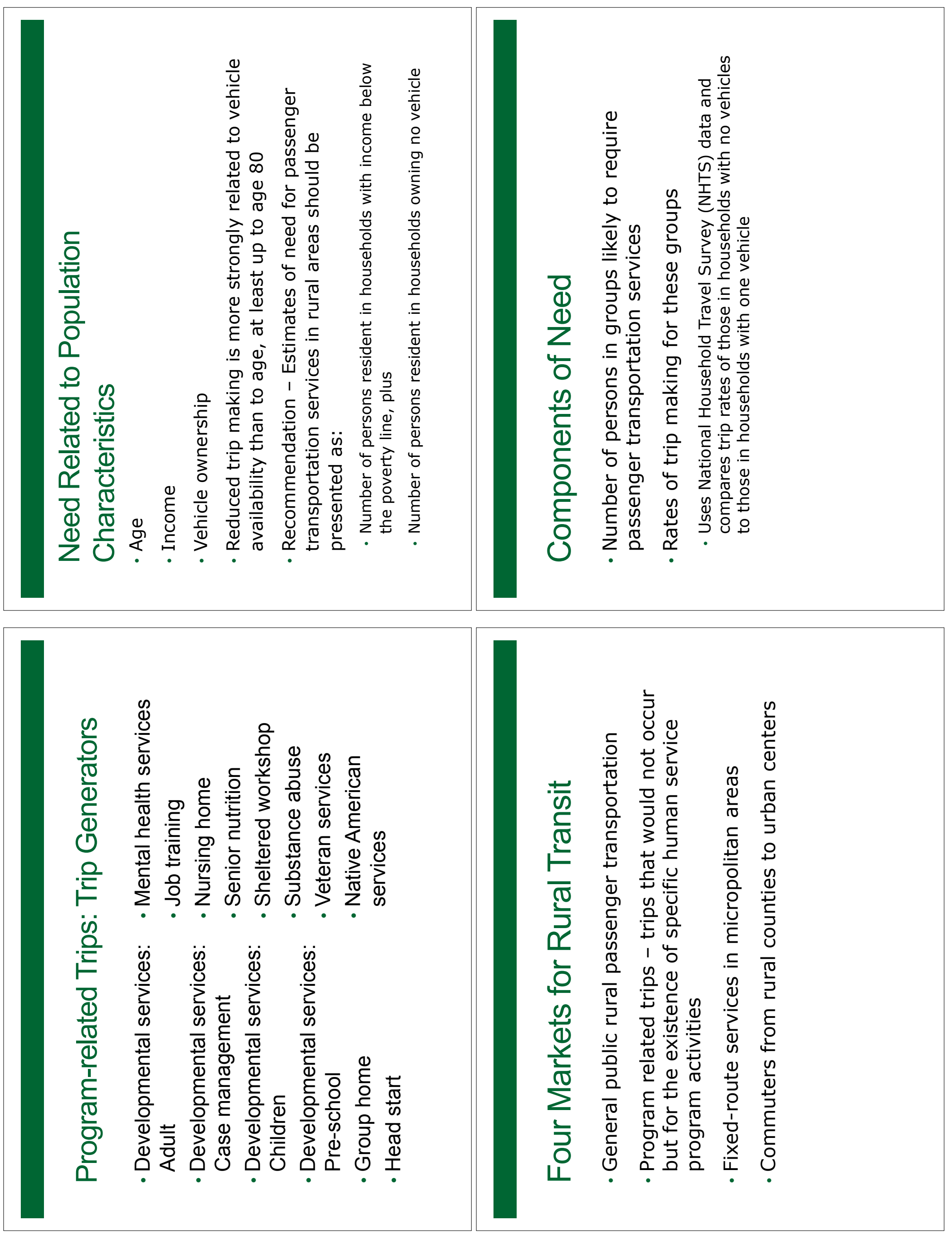


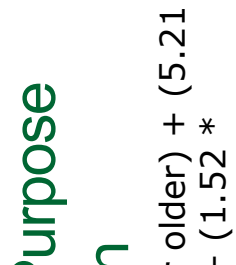
送

응ㅎㅇ

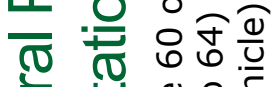

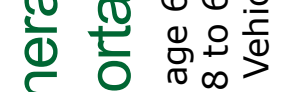

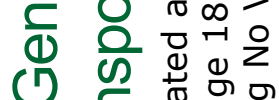

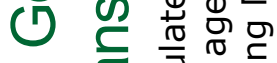

ช் 즈 믕.응

而

তে ⿺

(1) 으 응 응

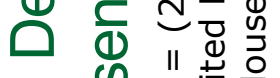
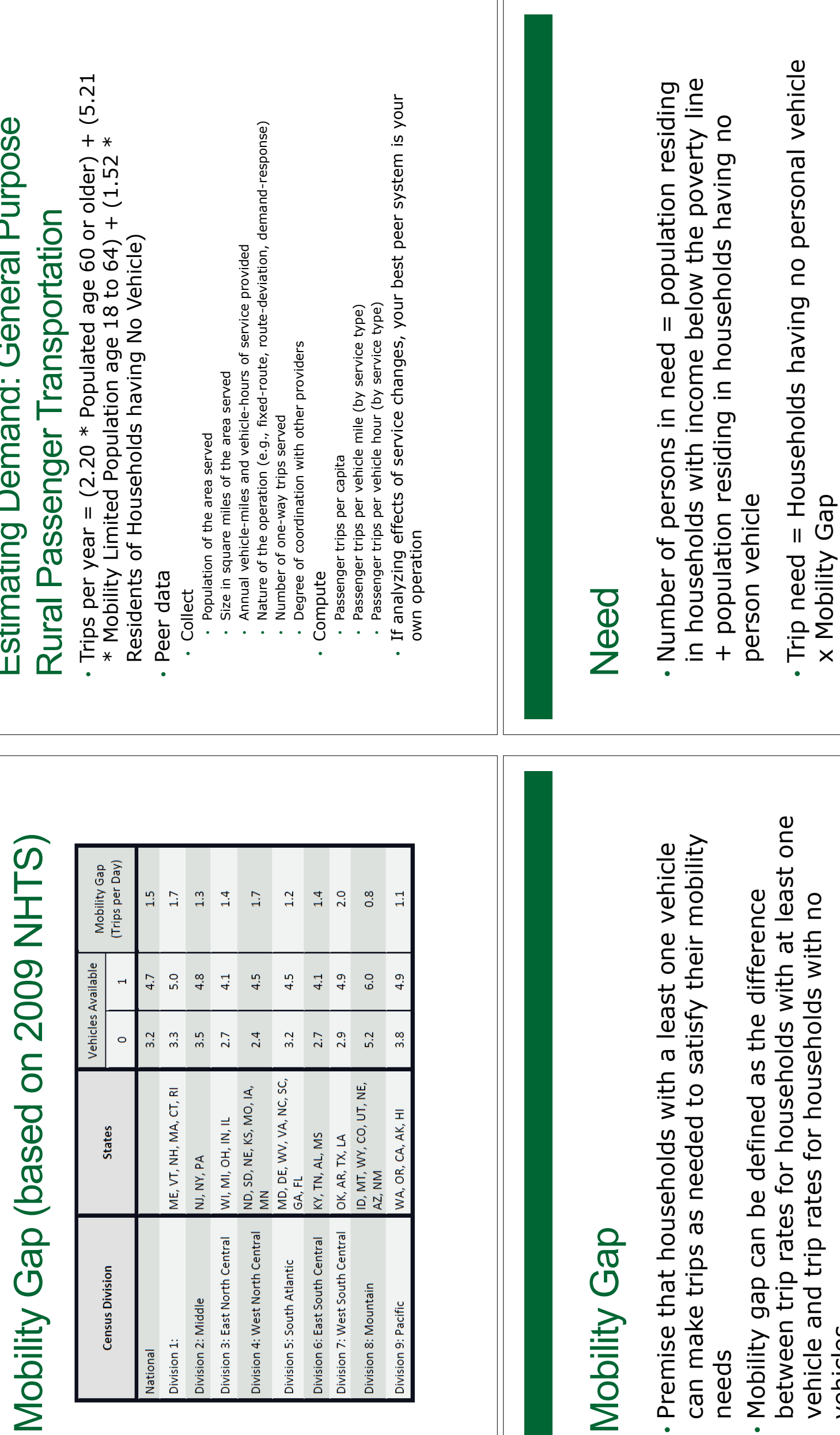

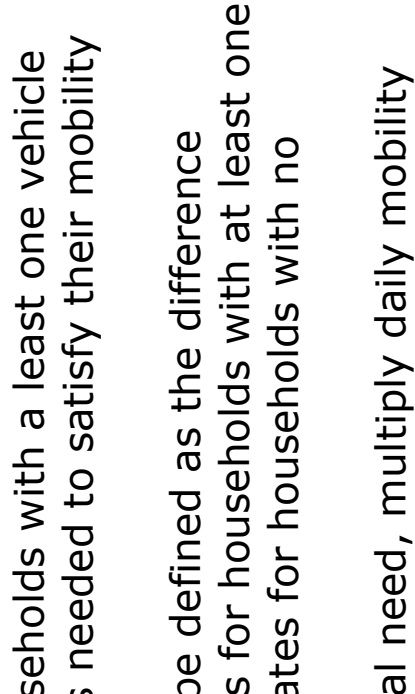

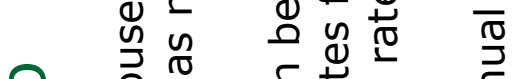

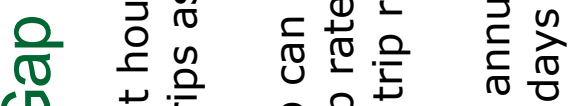

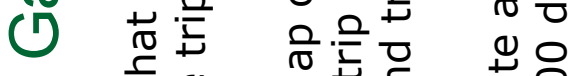

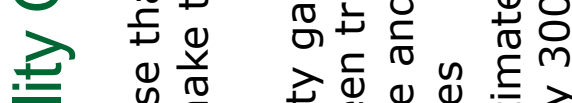

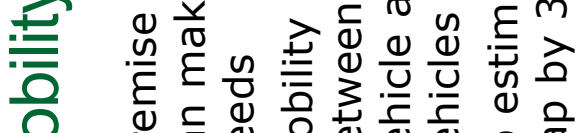

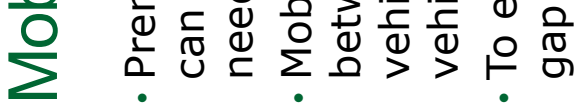




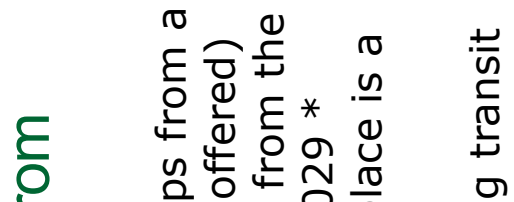

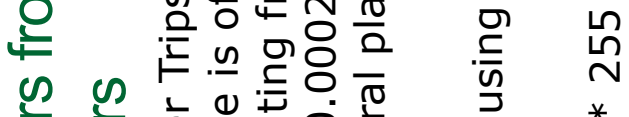

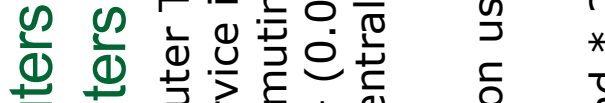

Ð

हல

○ी

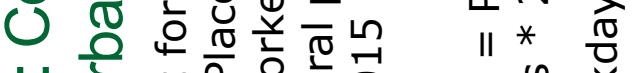

ช்

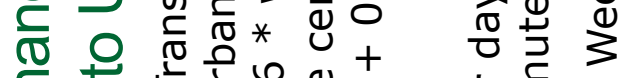

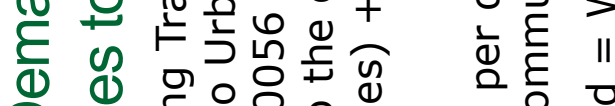

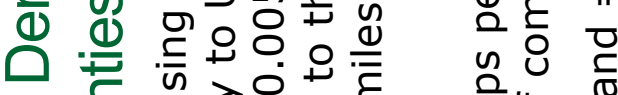

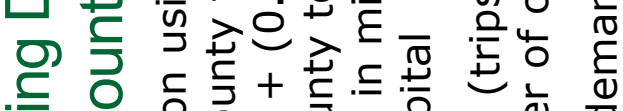

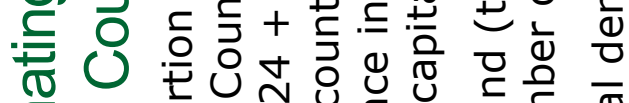

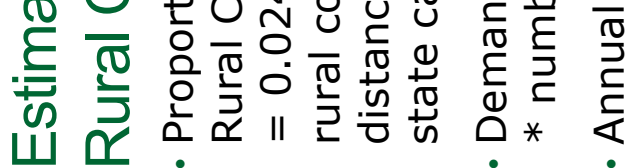

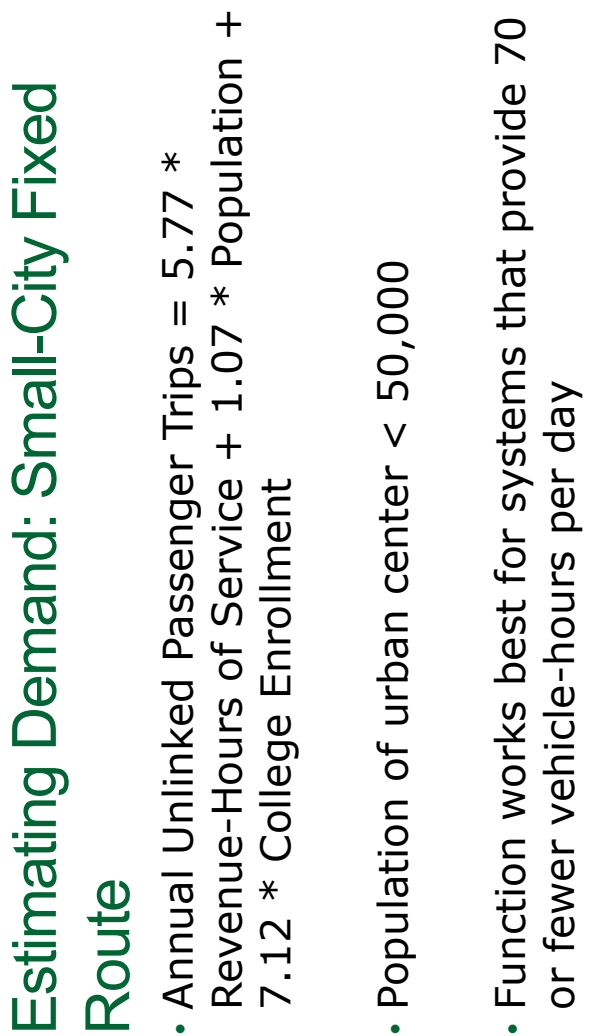

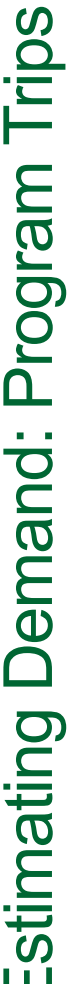
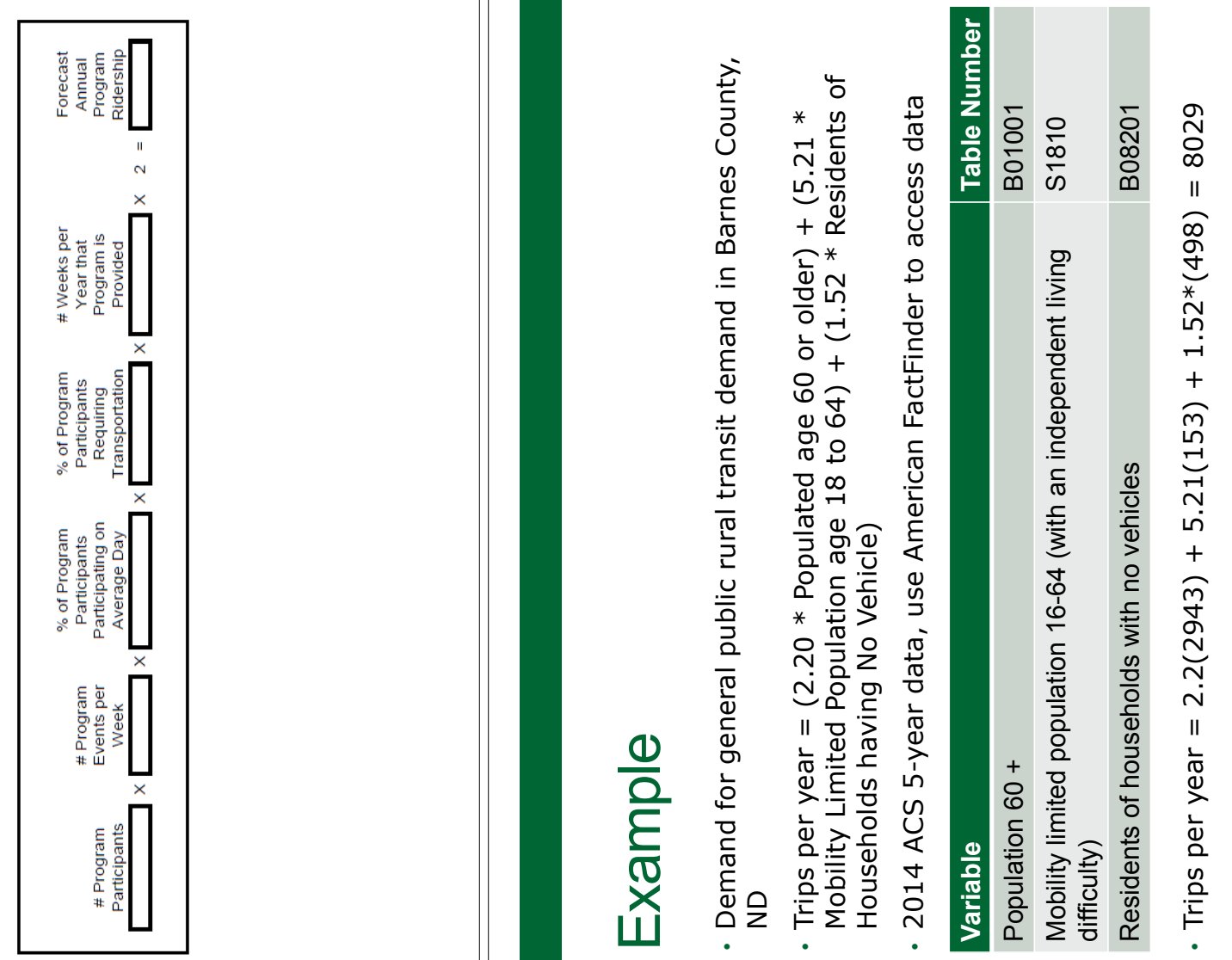

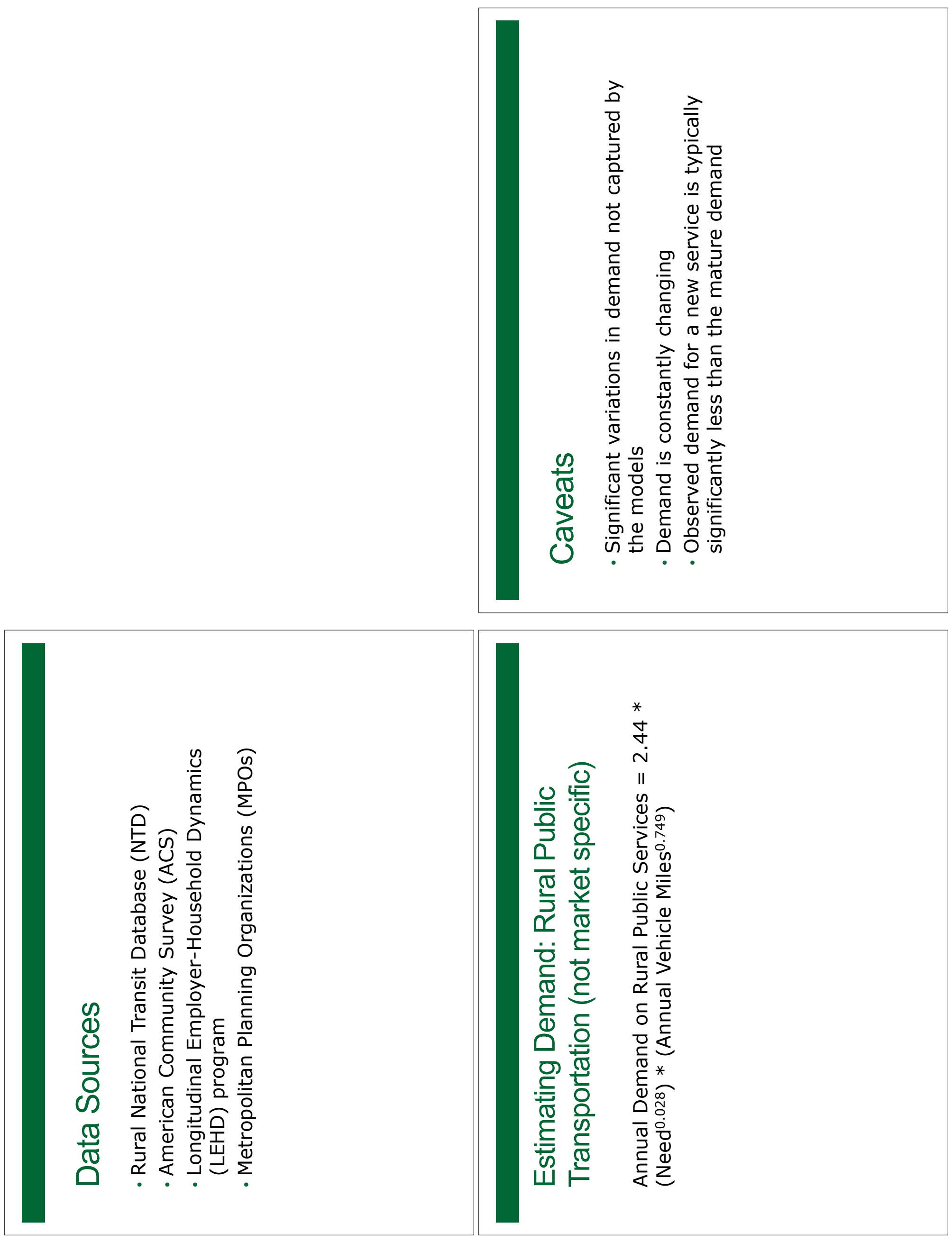

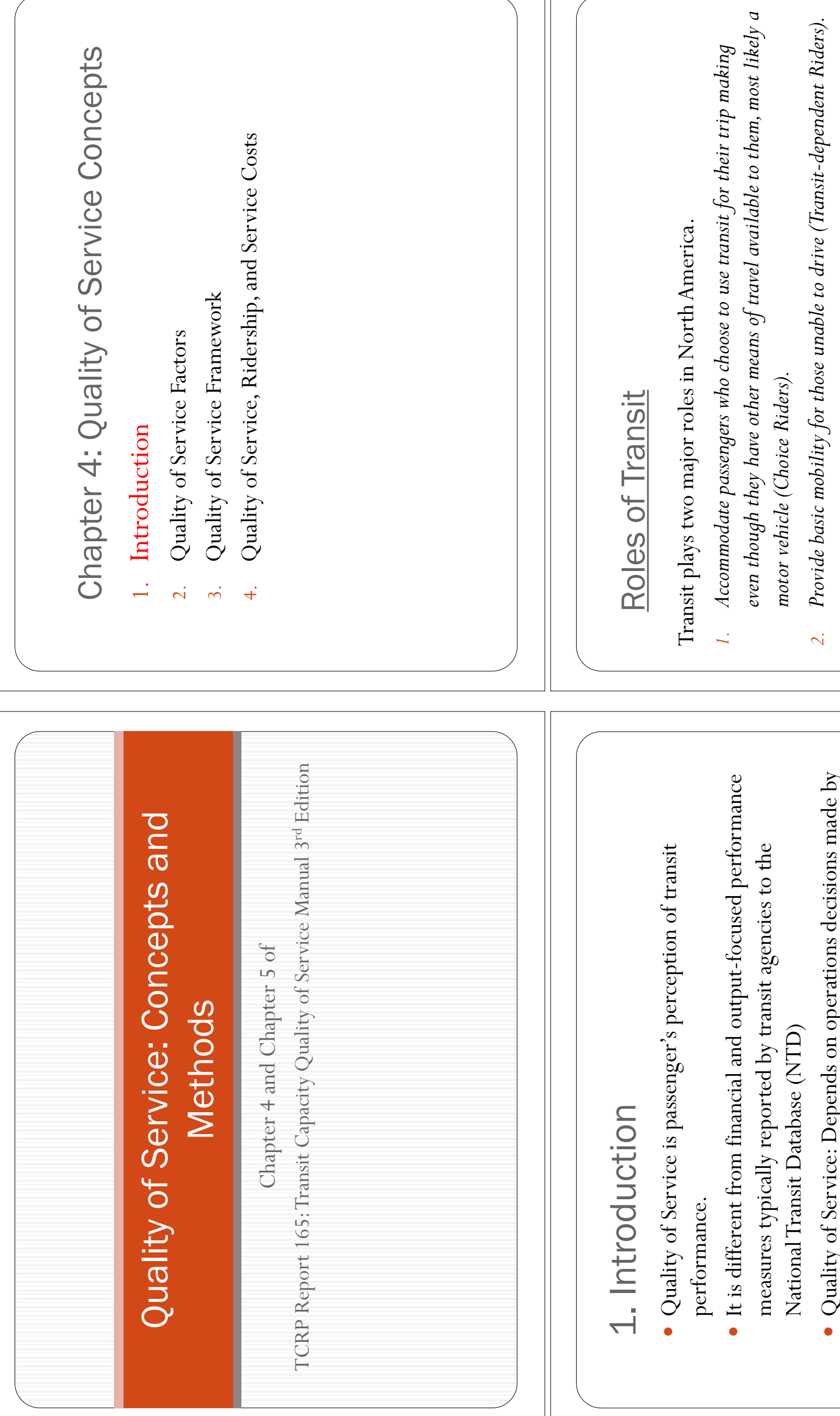

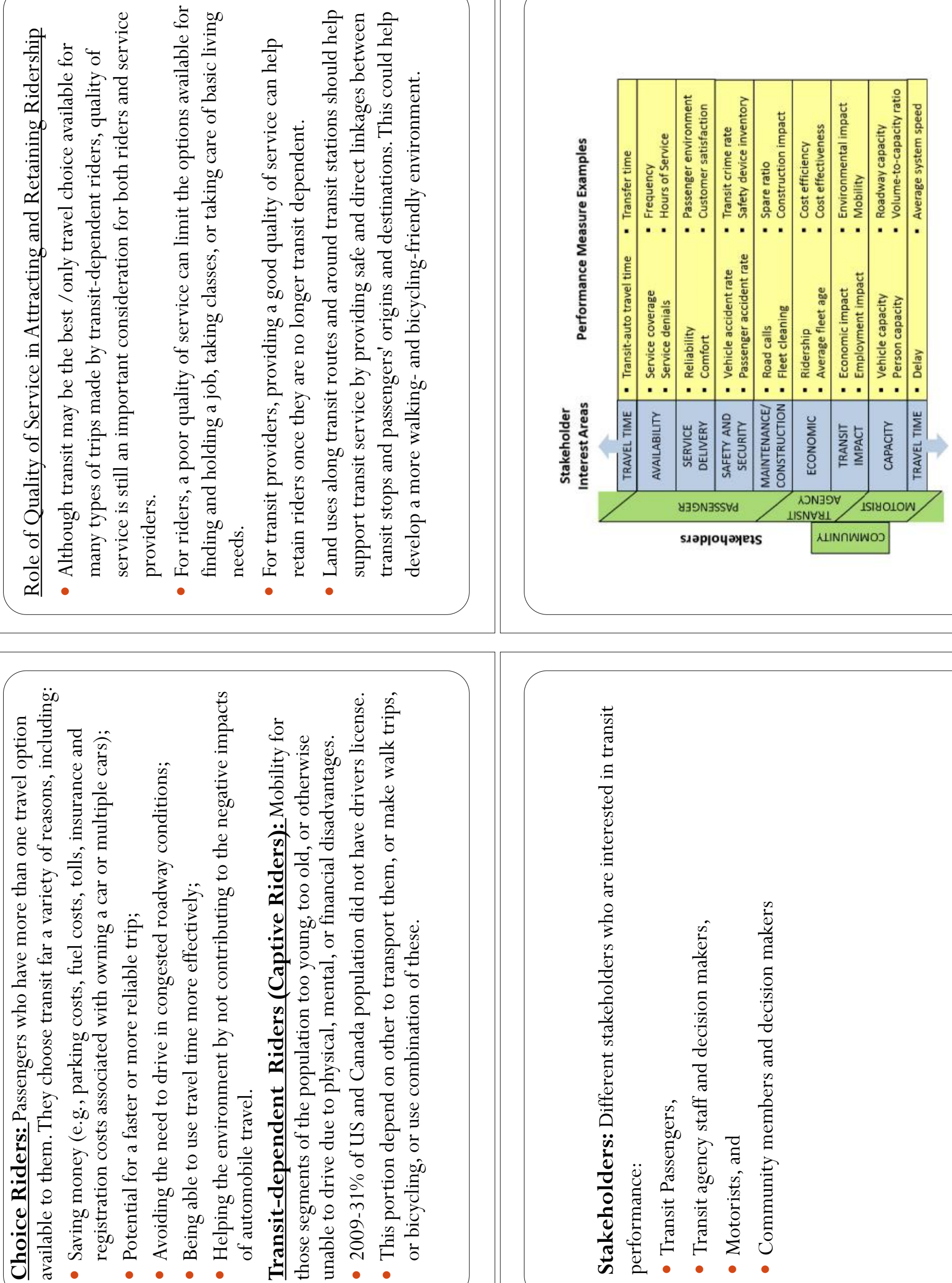

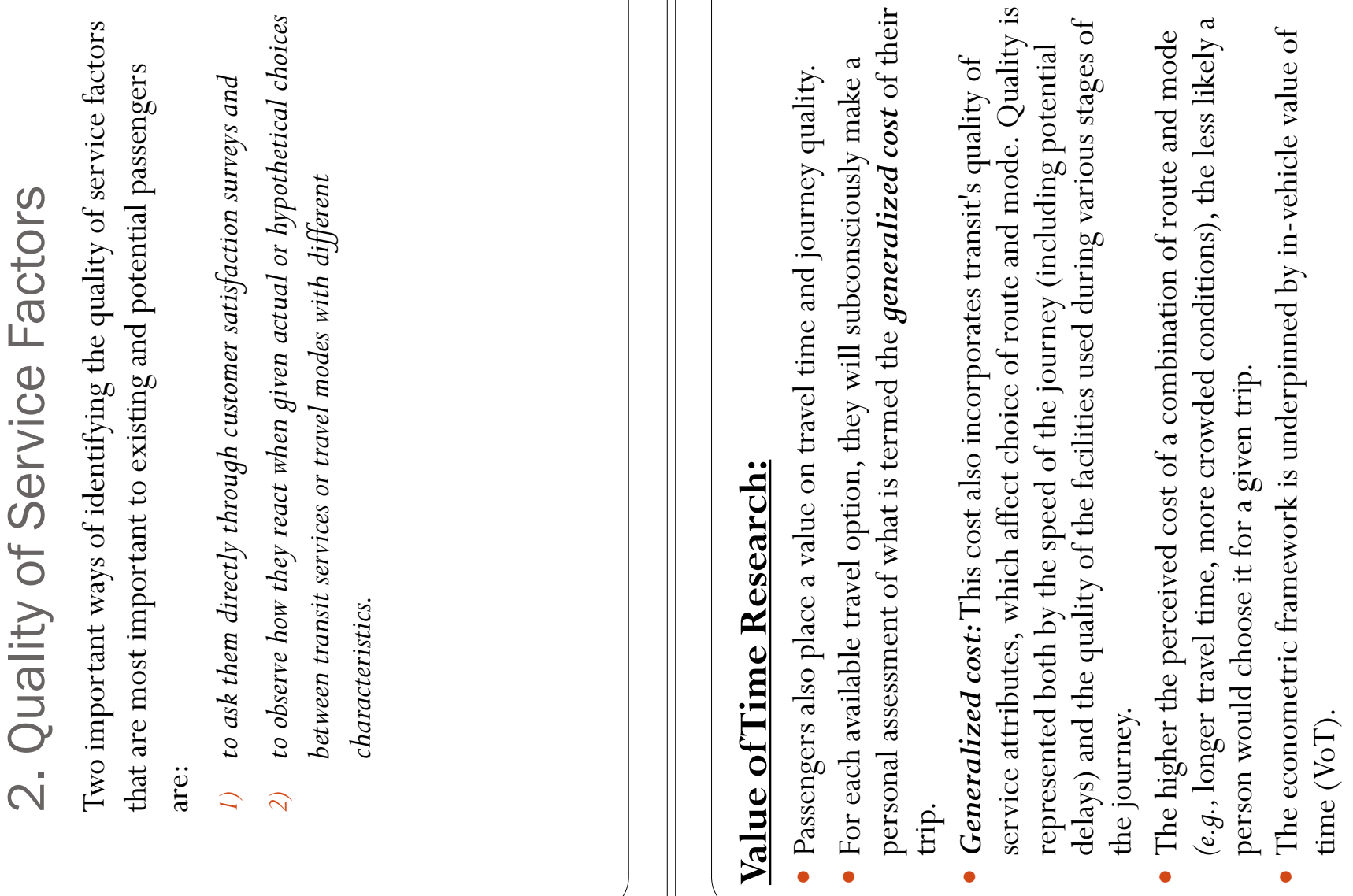

$\frac{0}{0}$
$\frac{1}{0}$
0
0
0

$0_{0}^{0}$

U

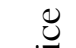

$\sum_{\infty}^{\frac{1}{2}}$ 岕

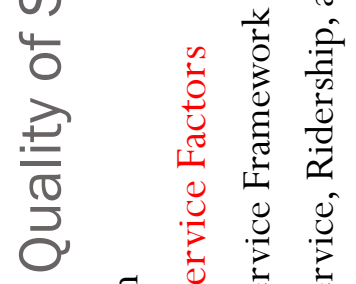

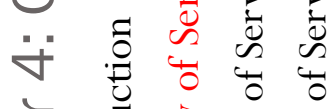

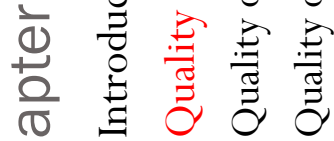

$\bar{\Xi}-\dot{i} \dot{ }$

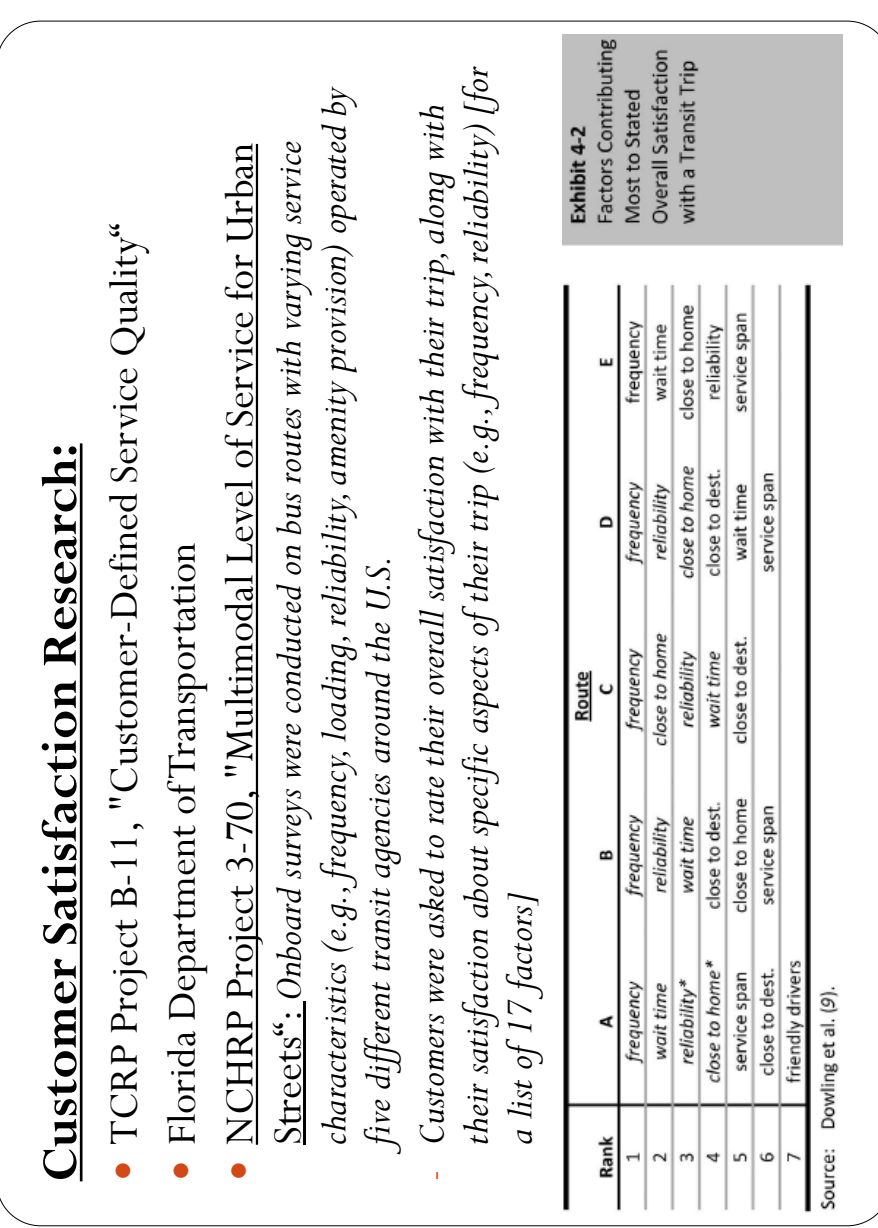



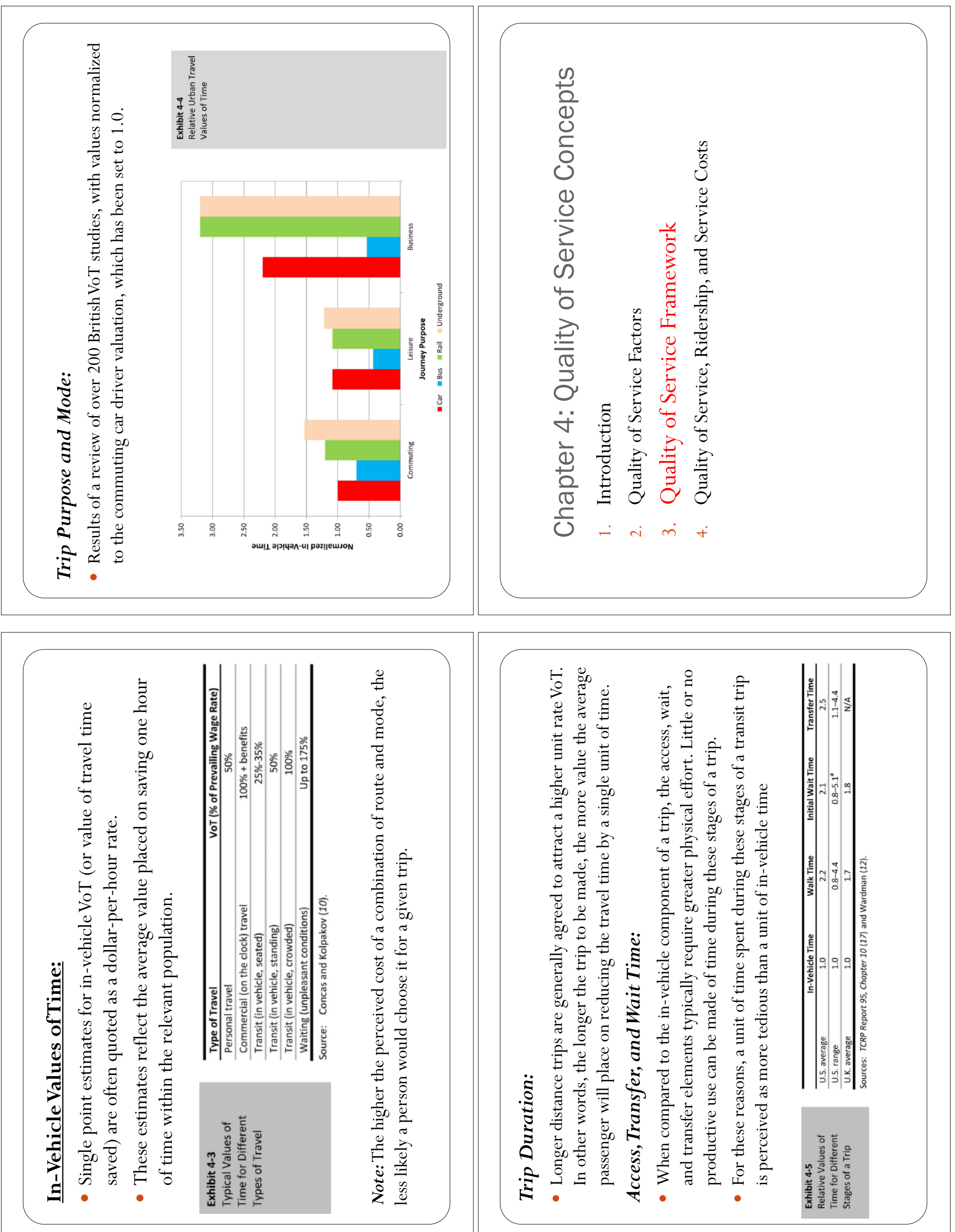

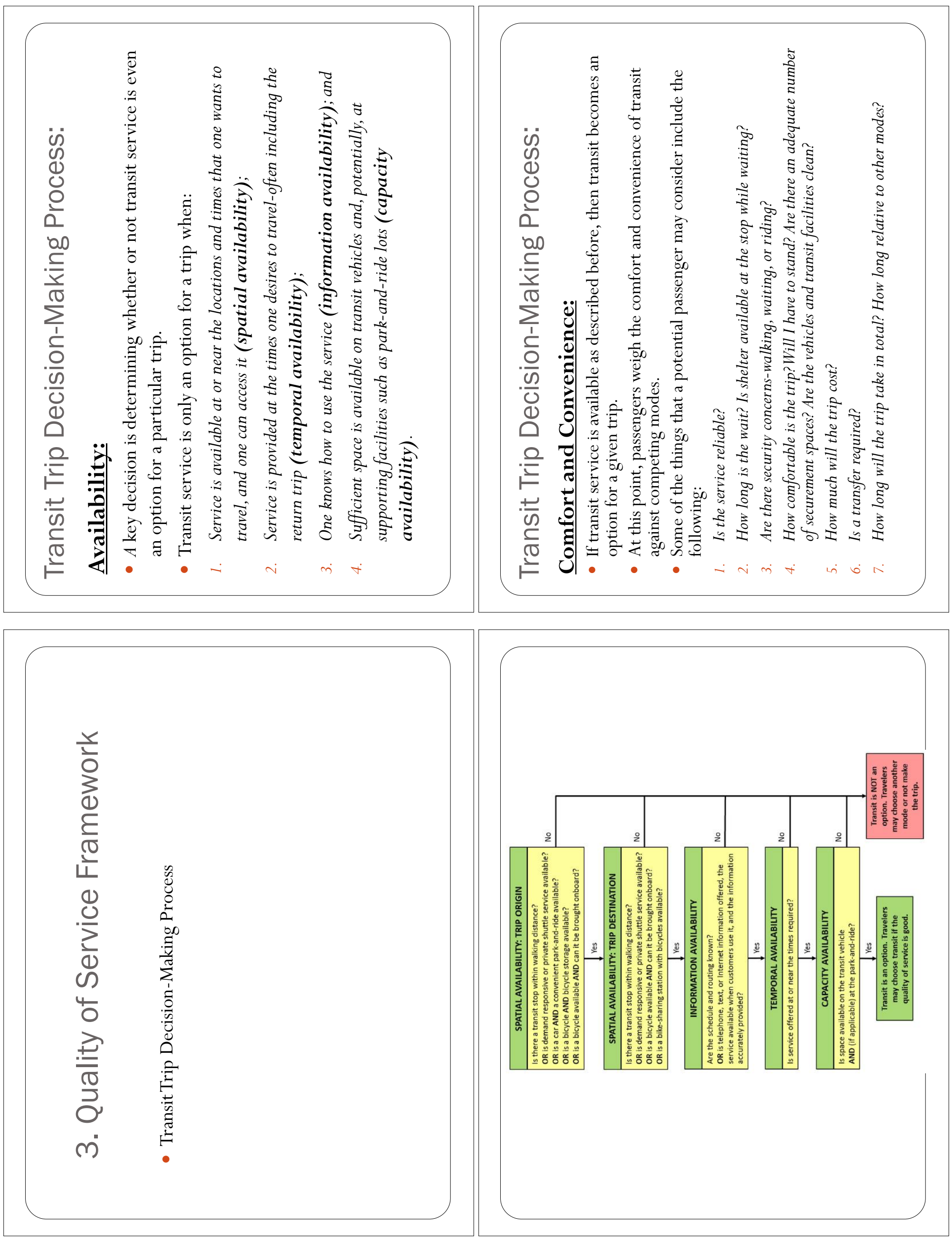

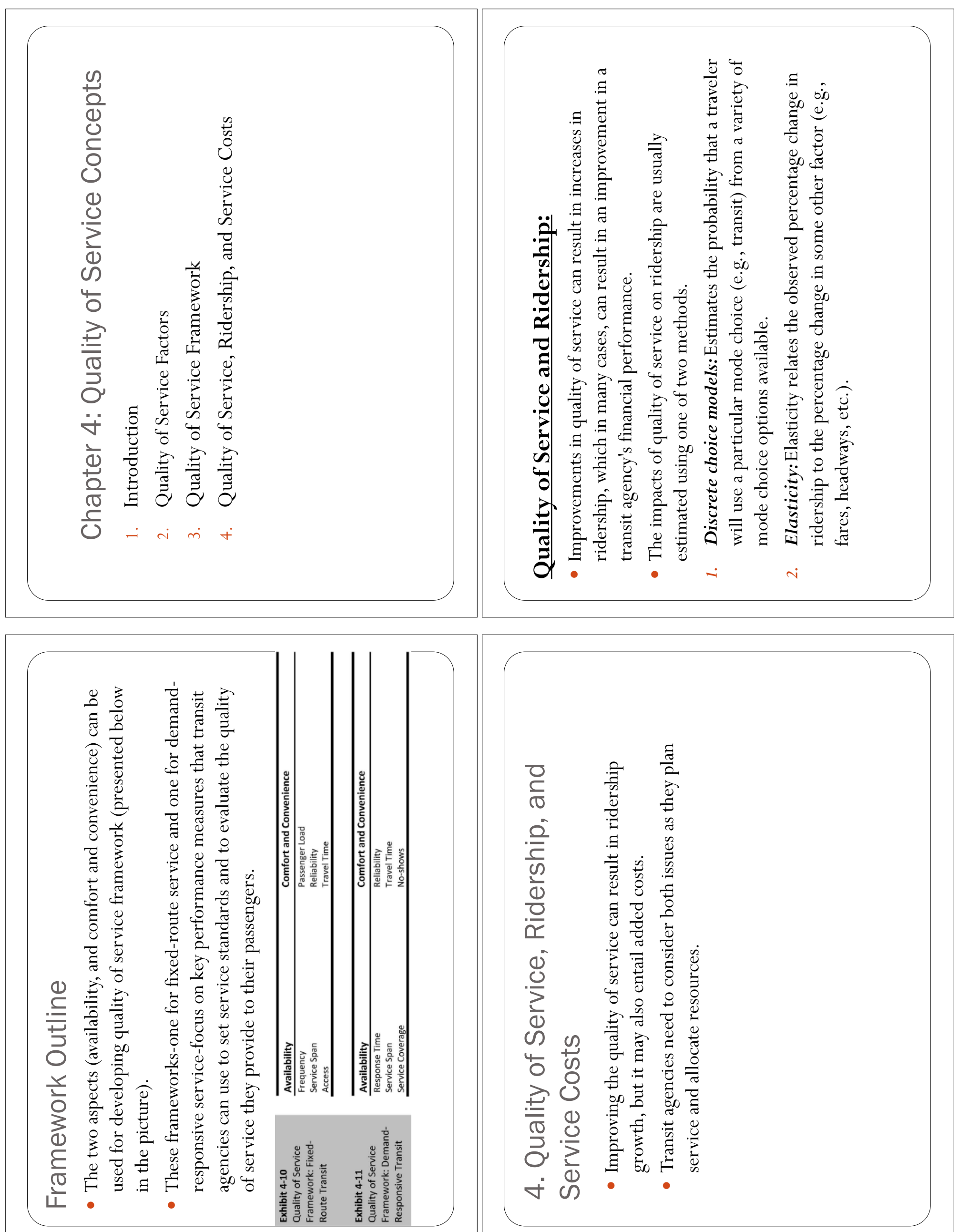

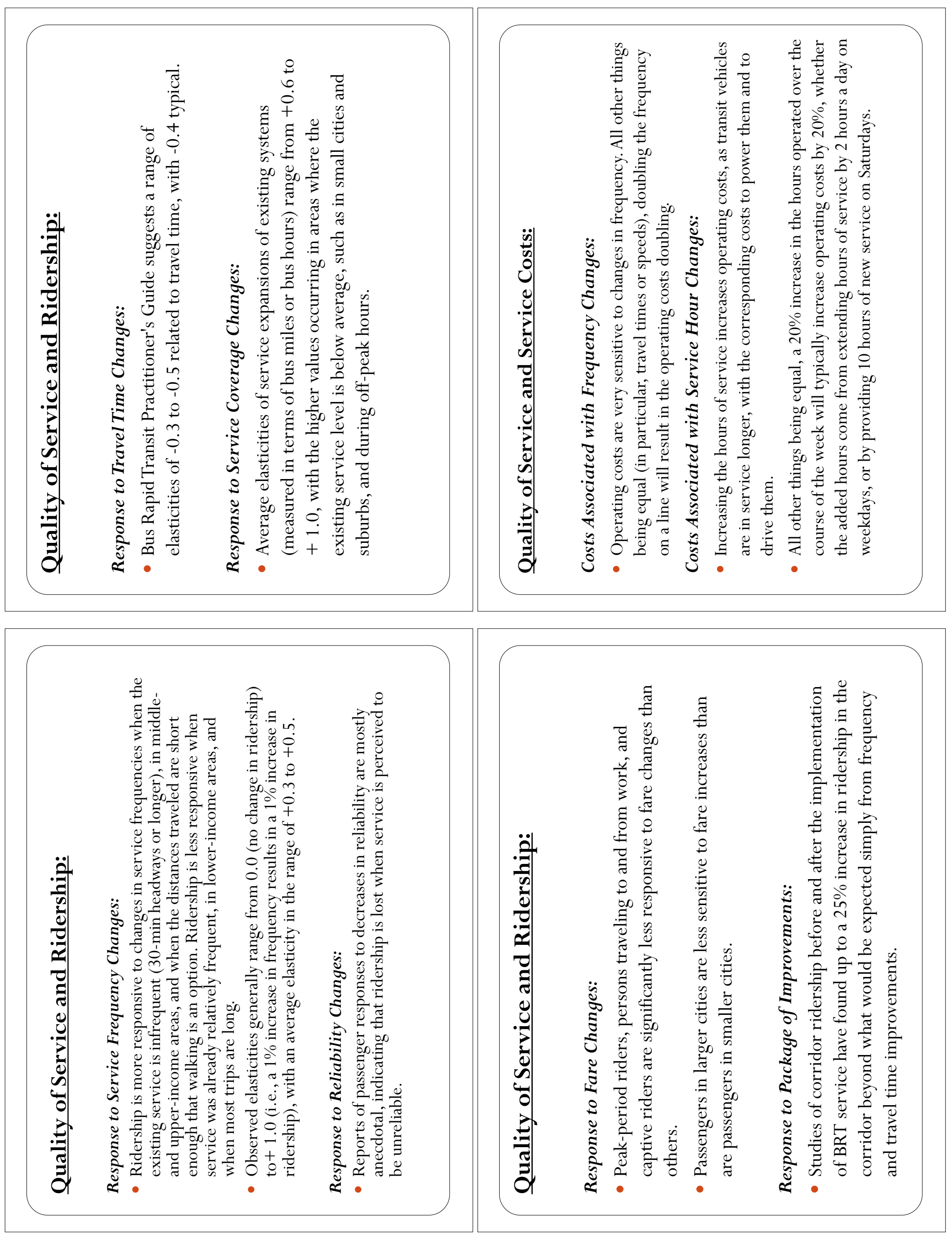

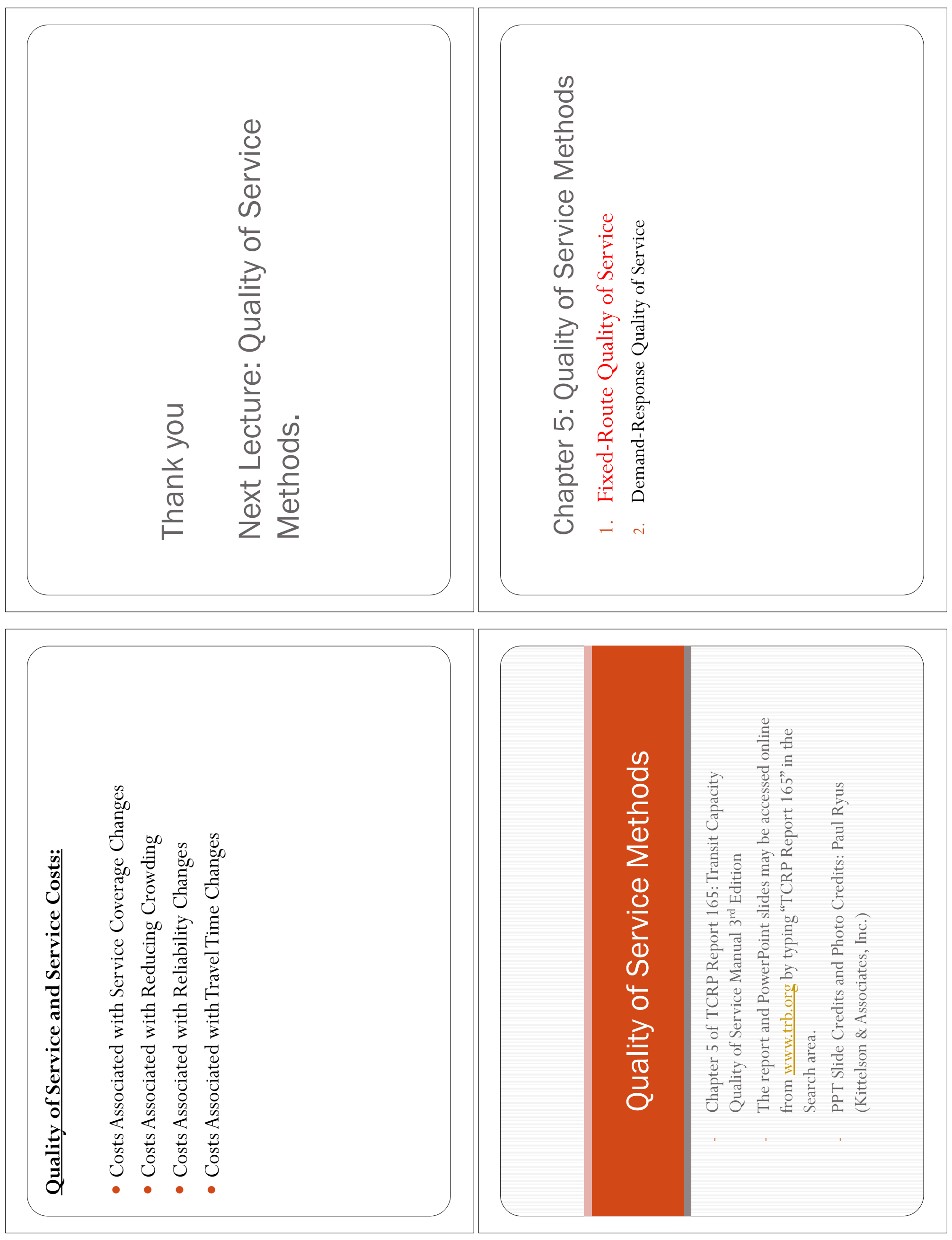

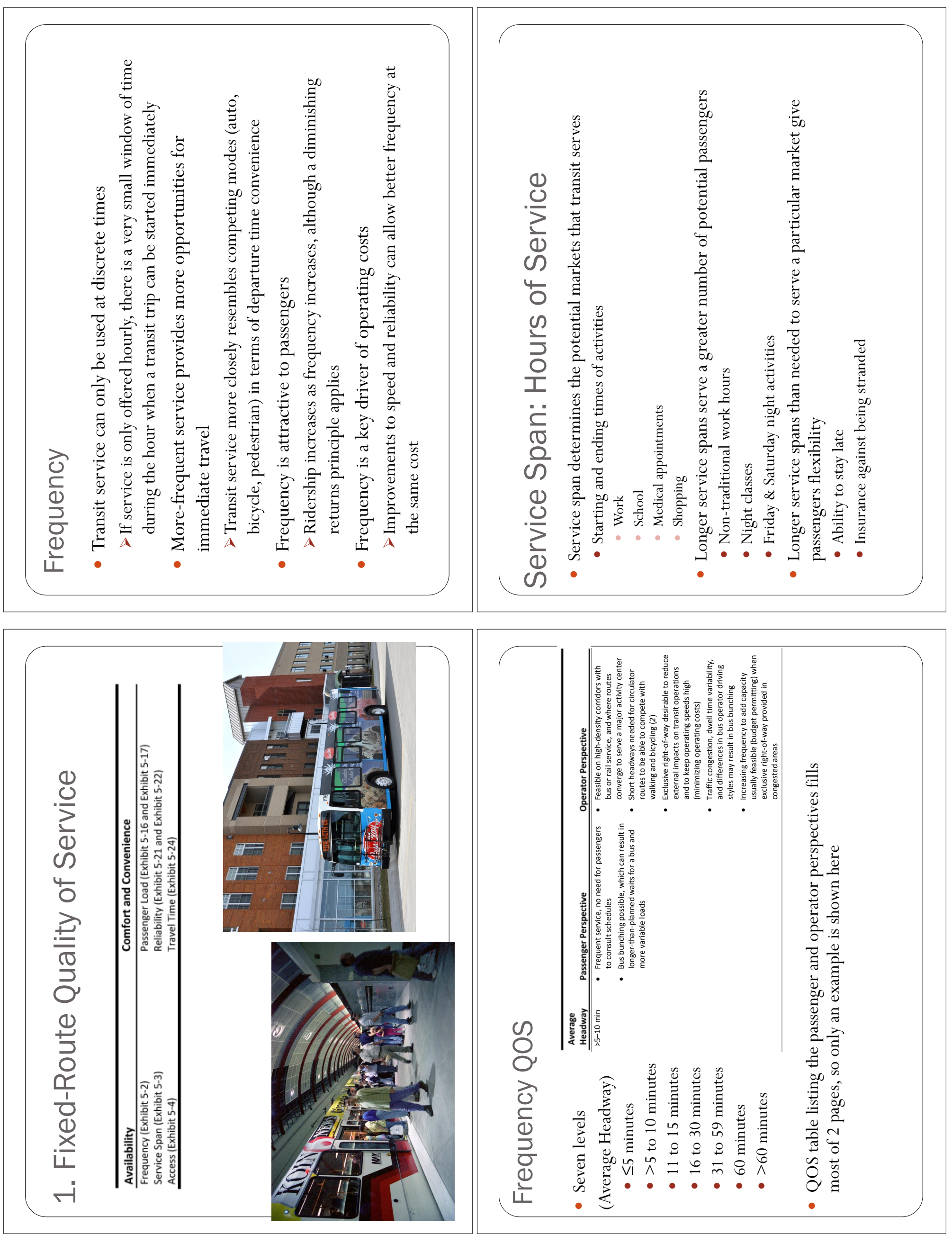

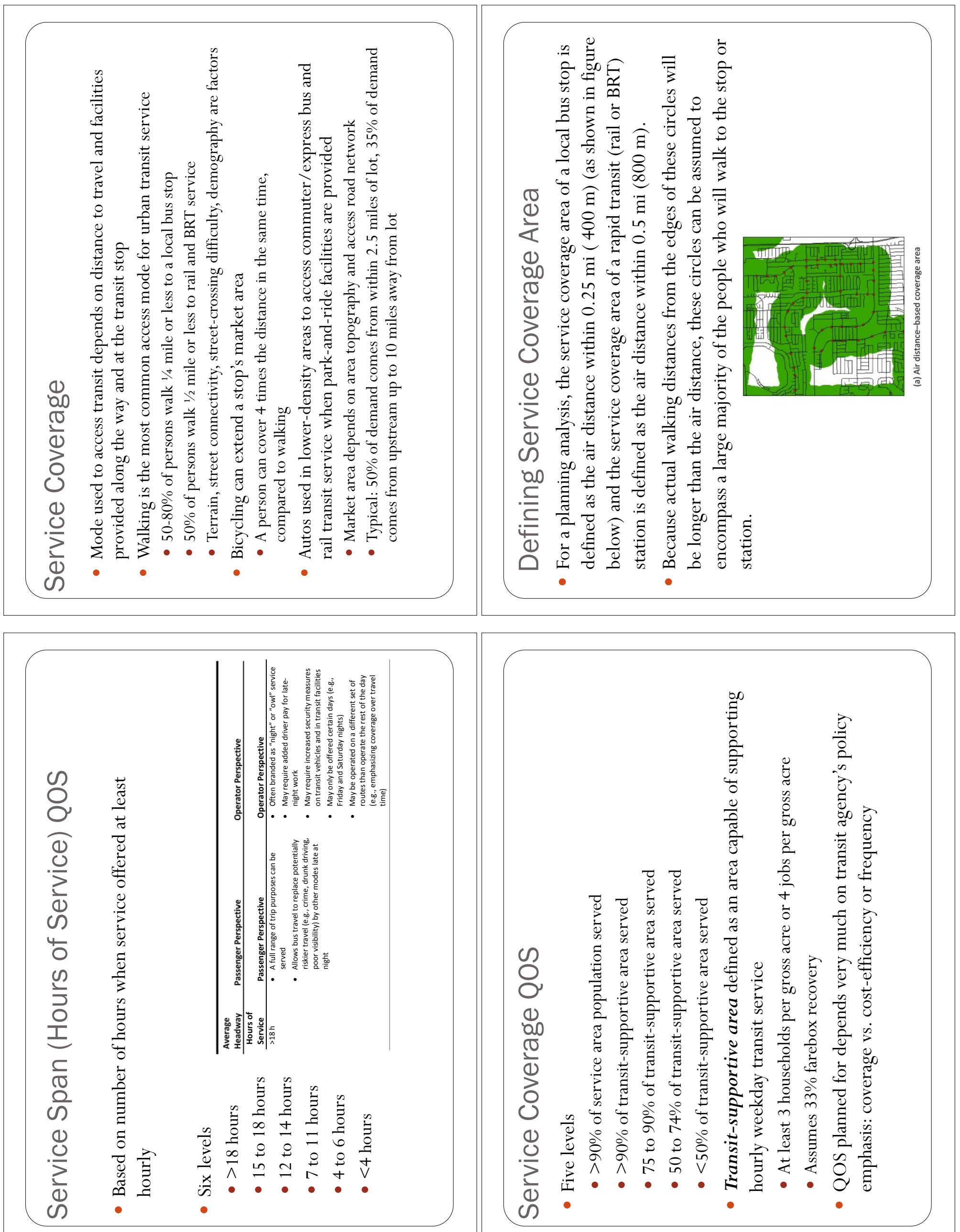

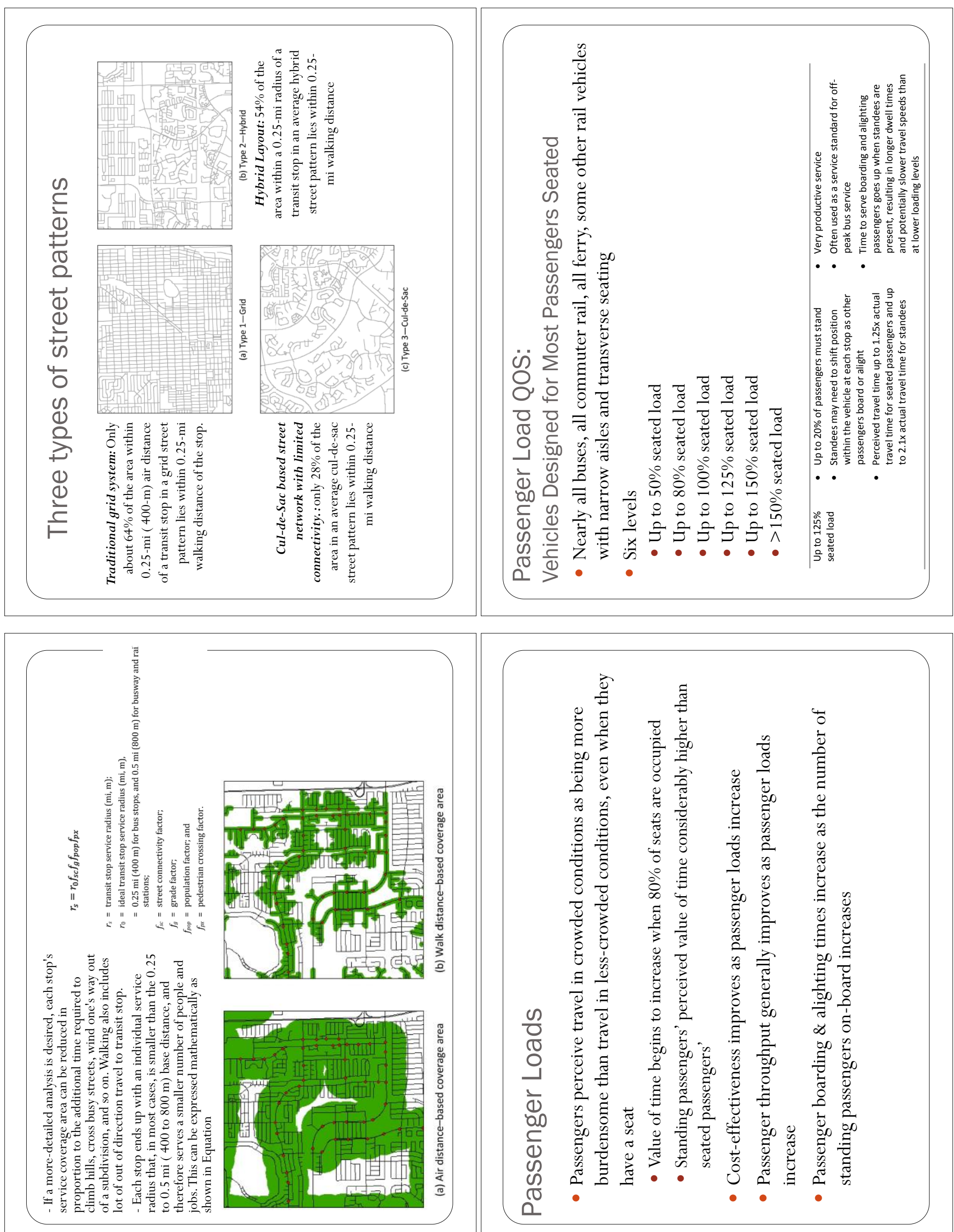

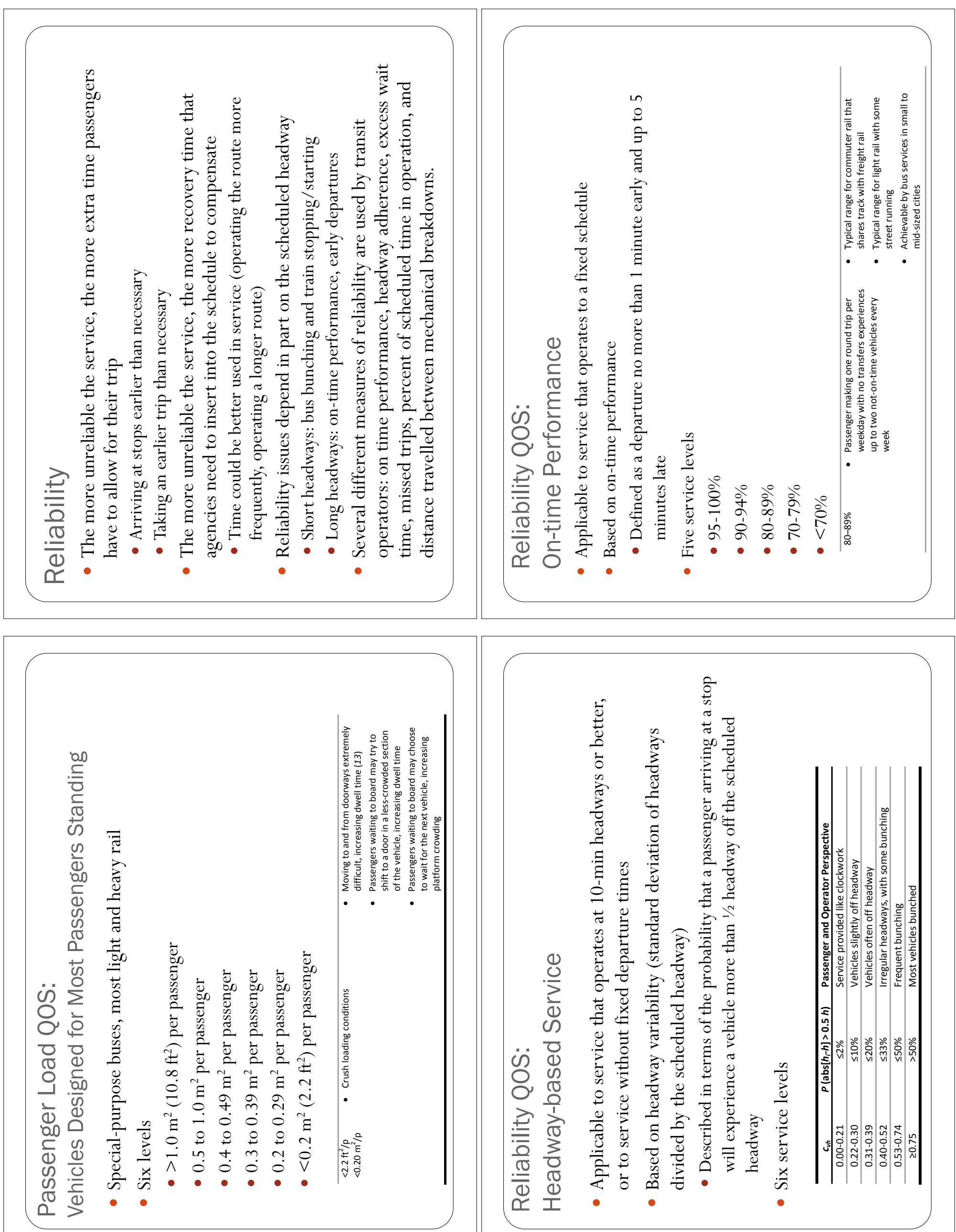

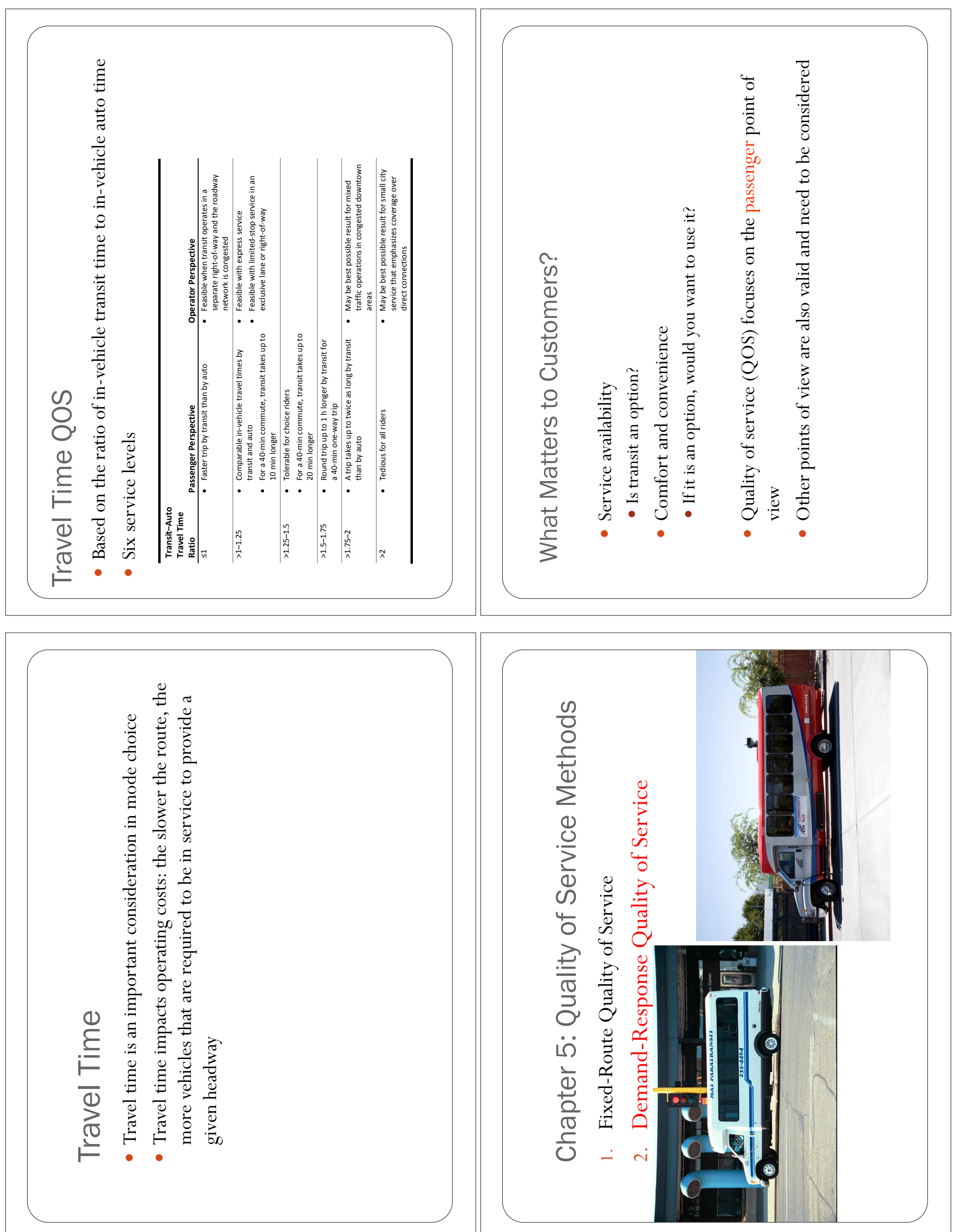

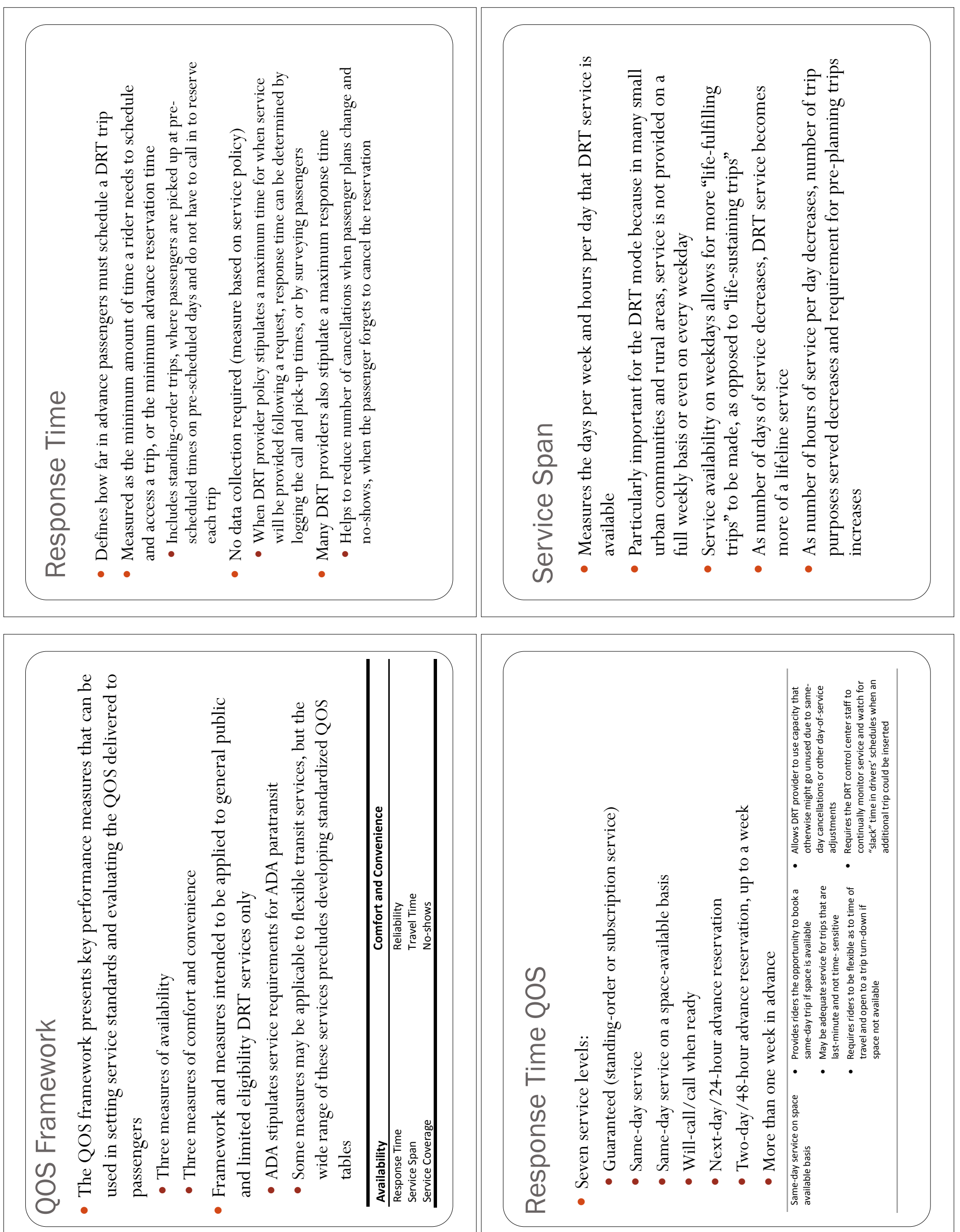

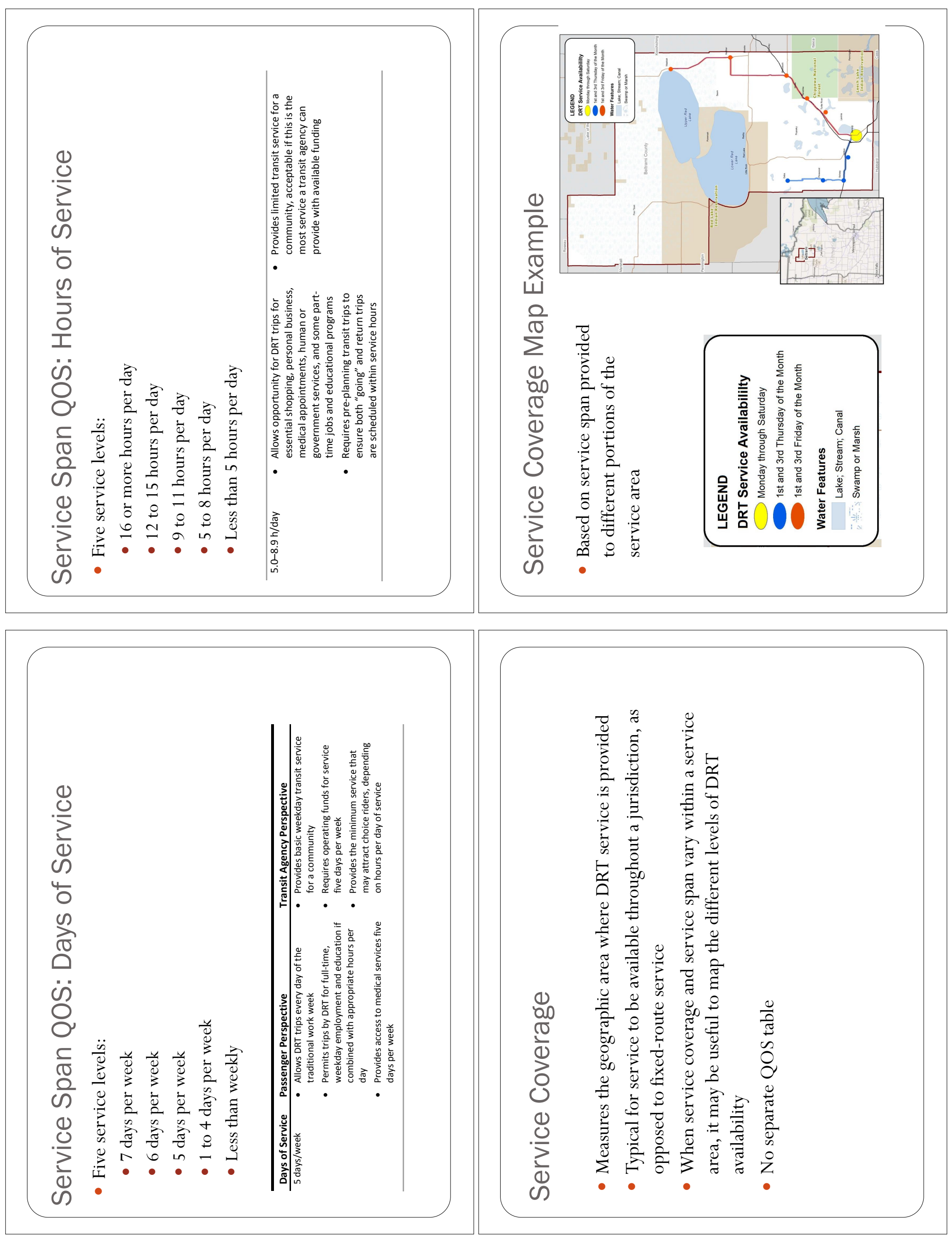

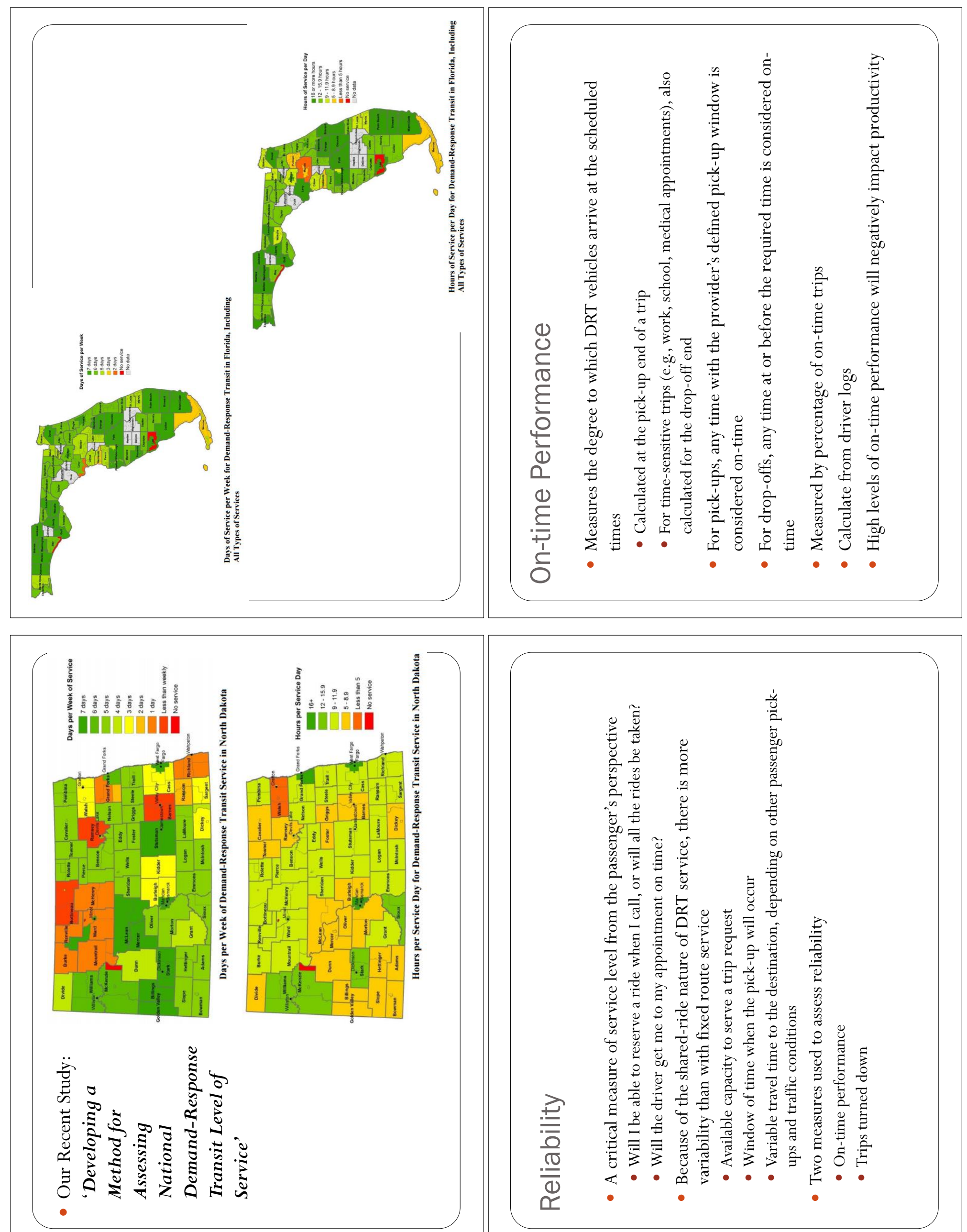

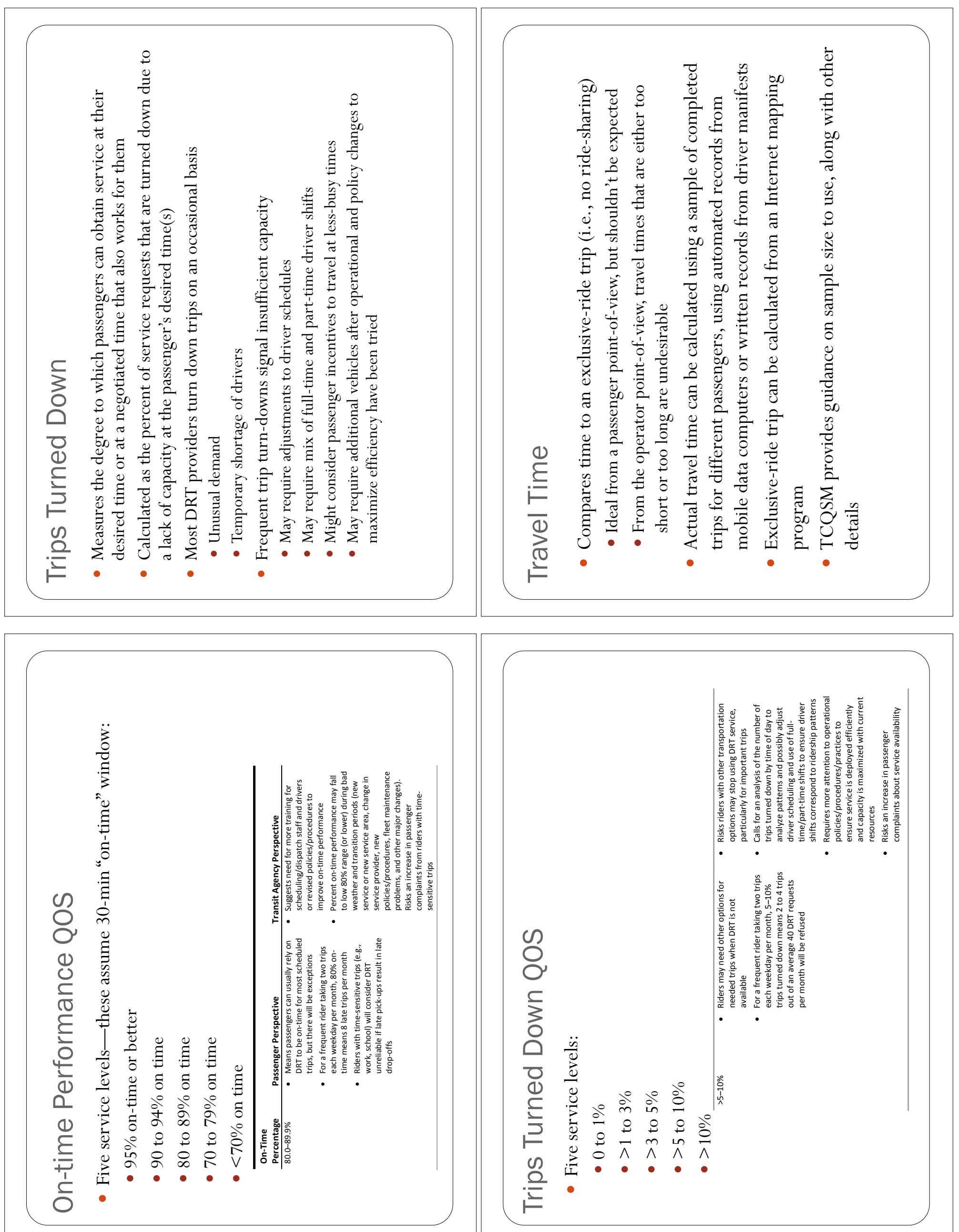

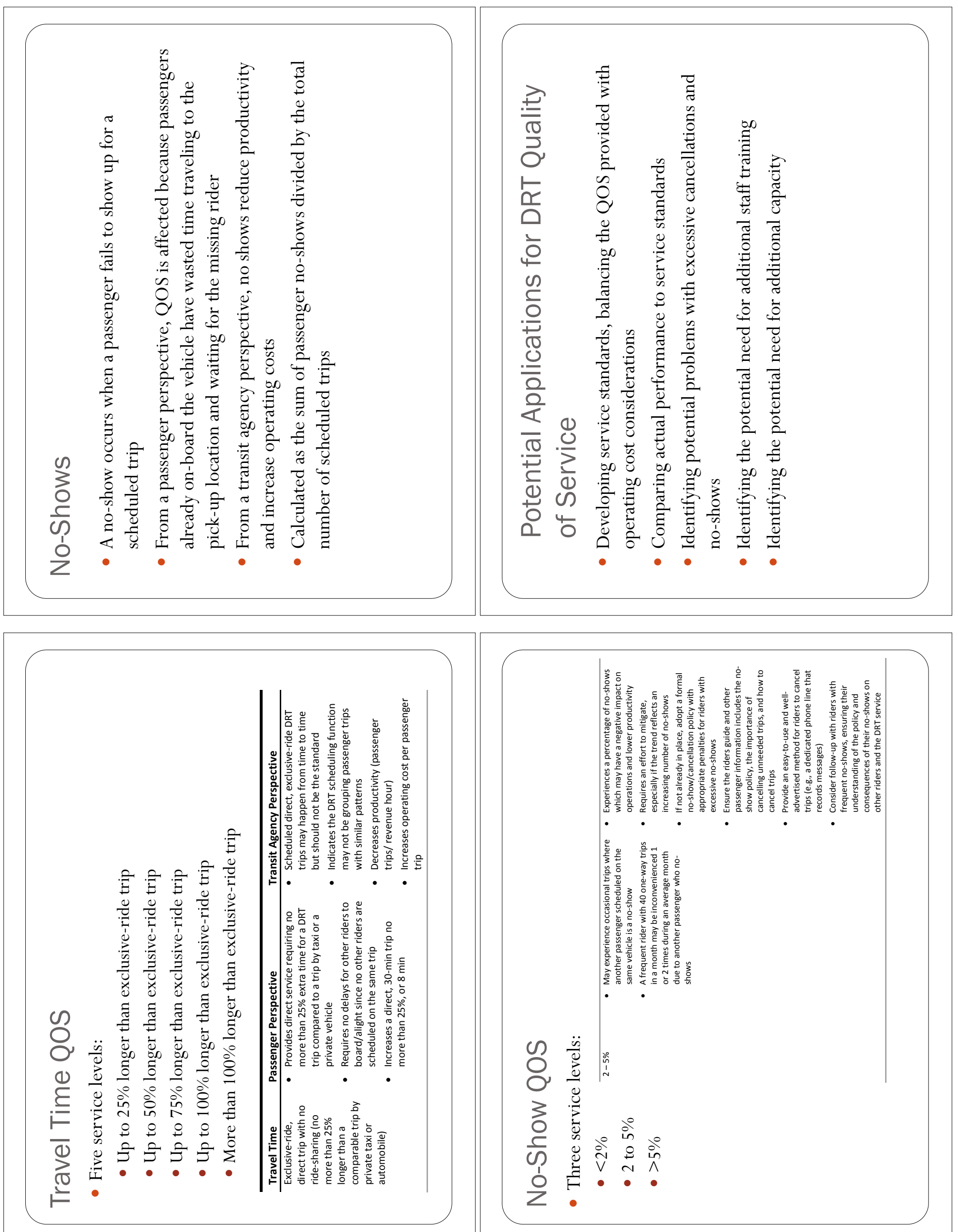


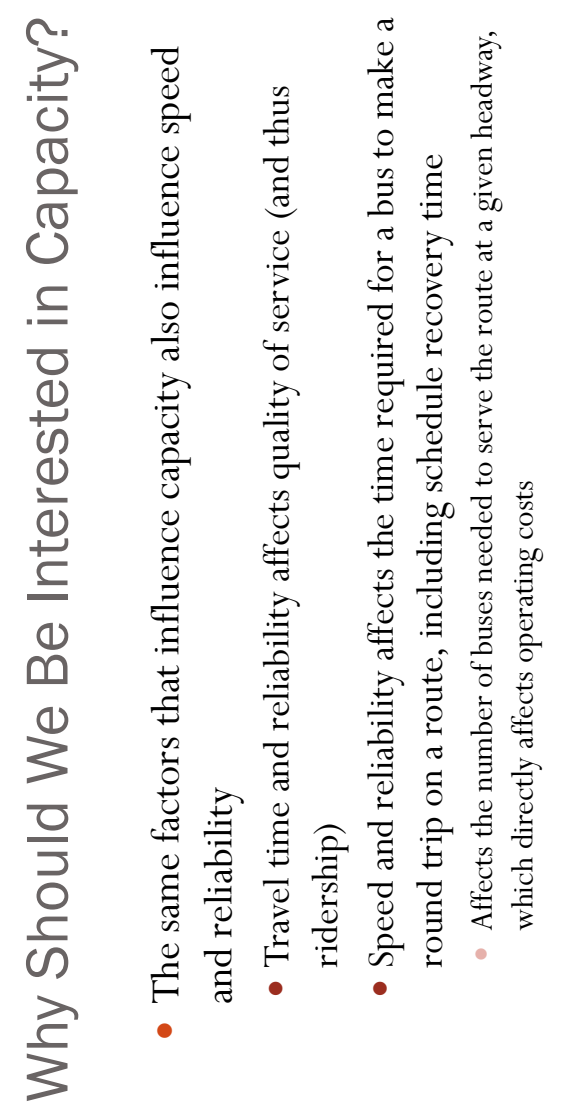

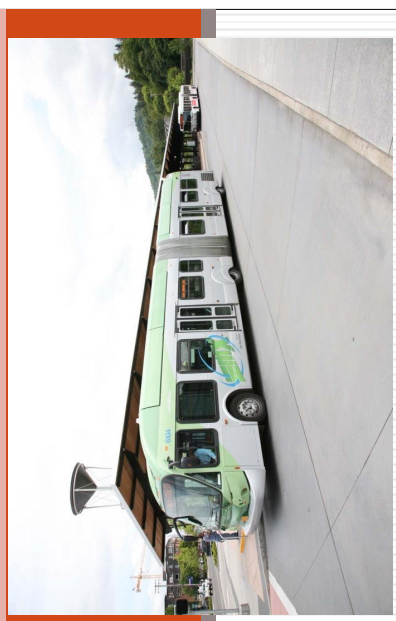

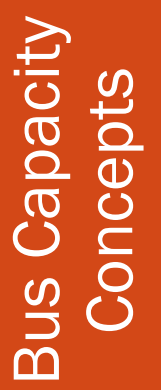

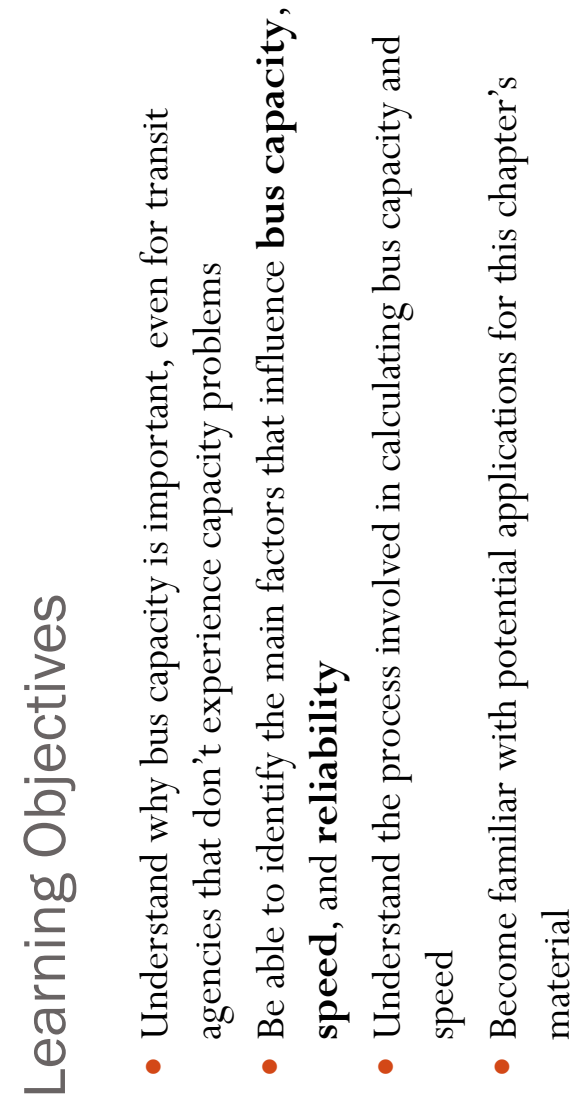



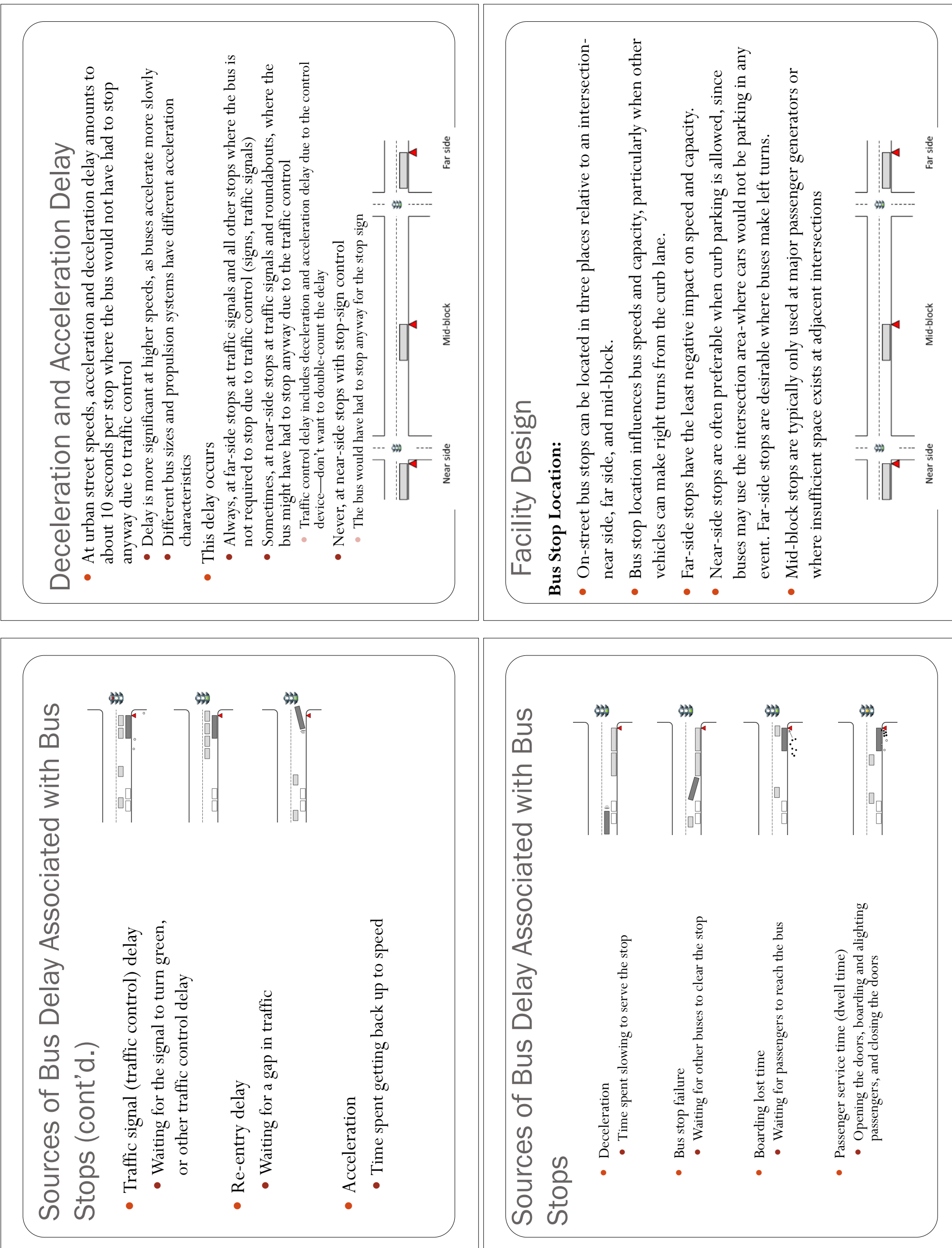

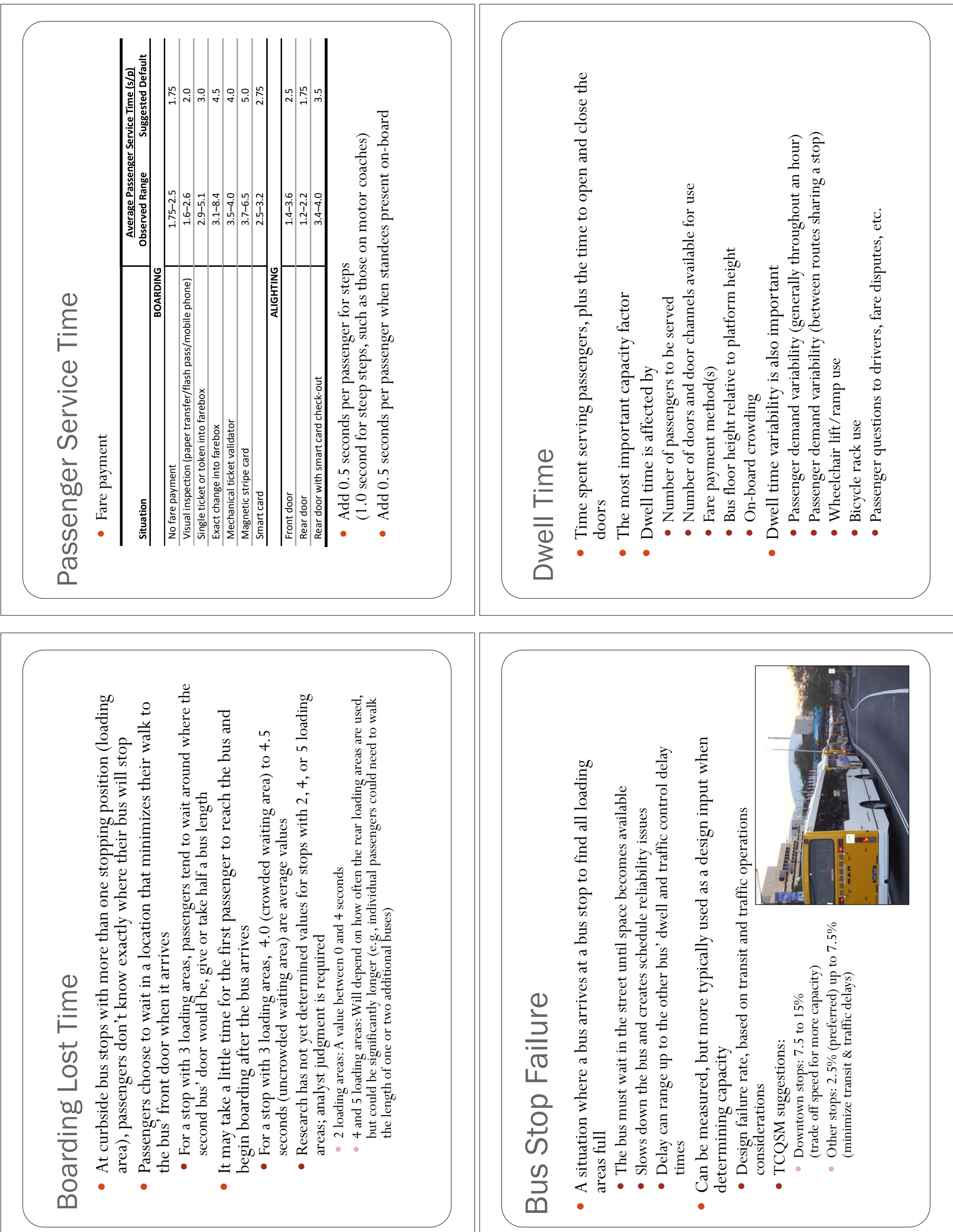

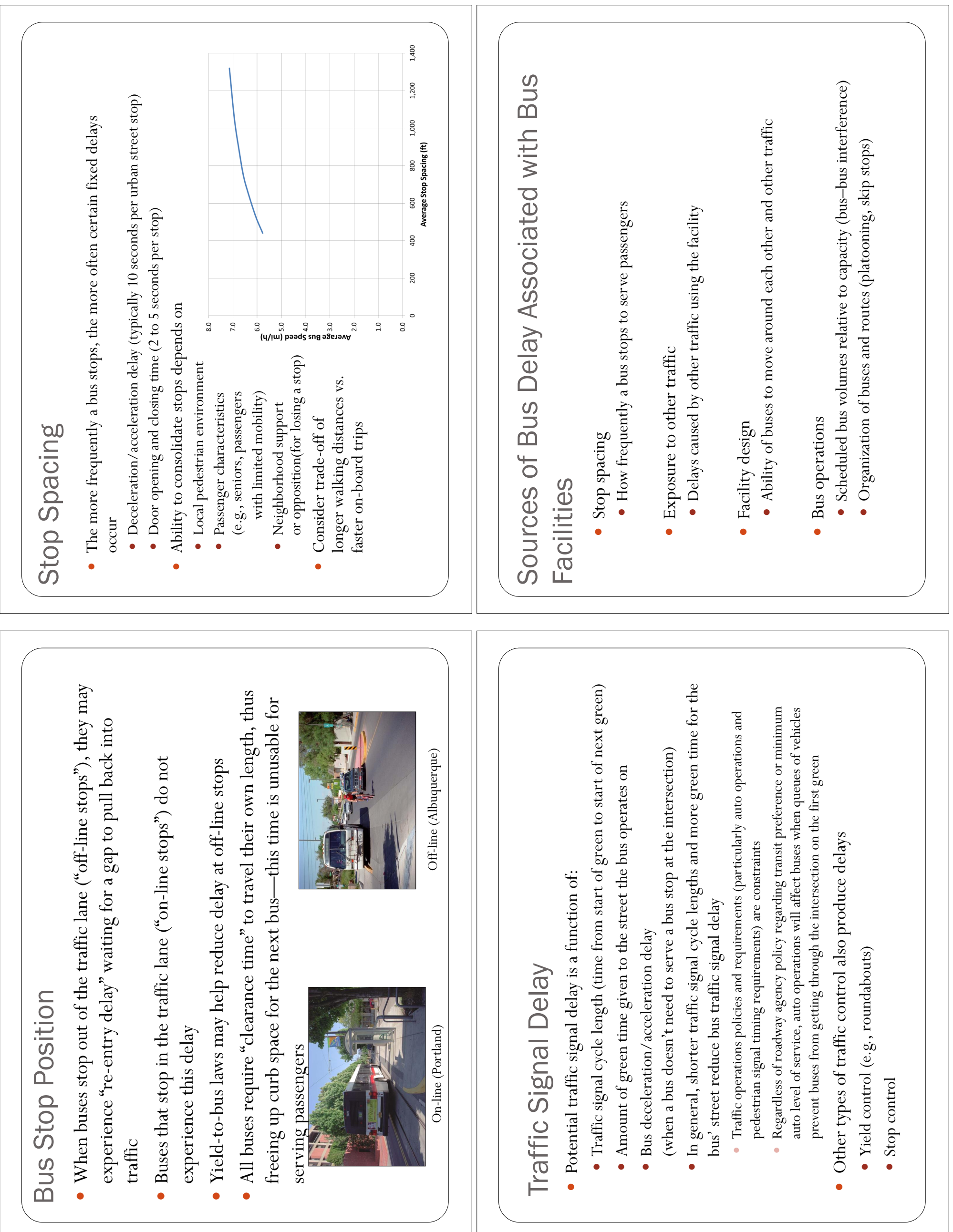

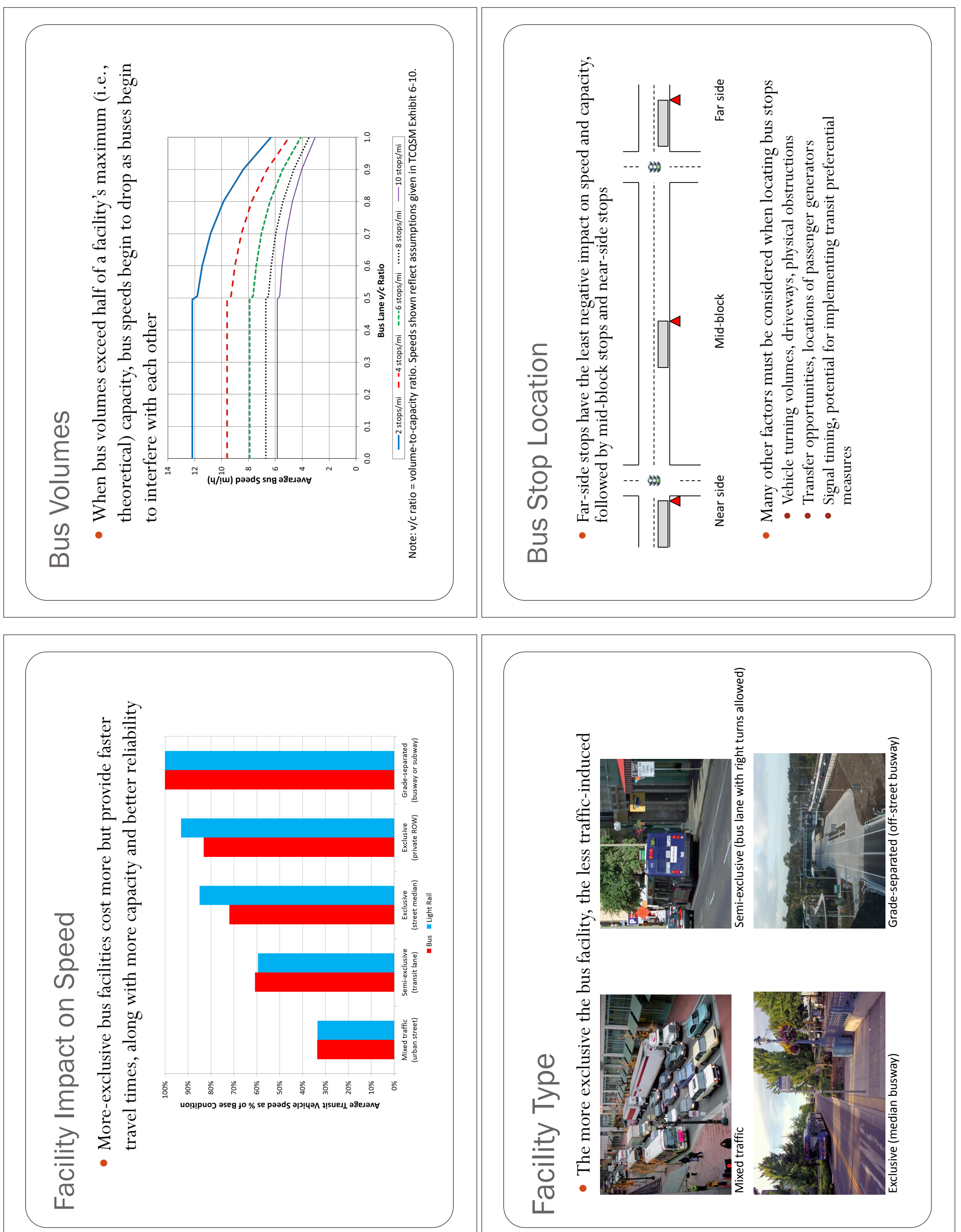

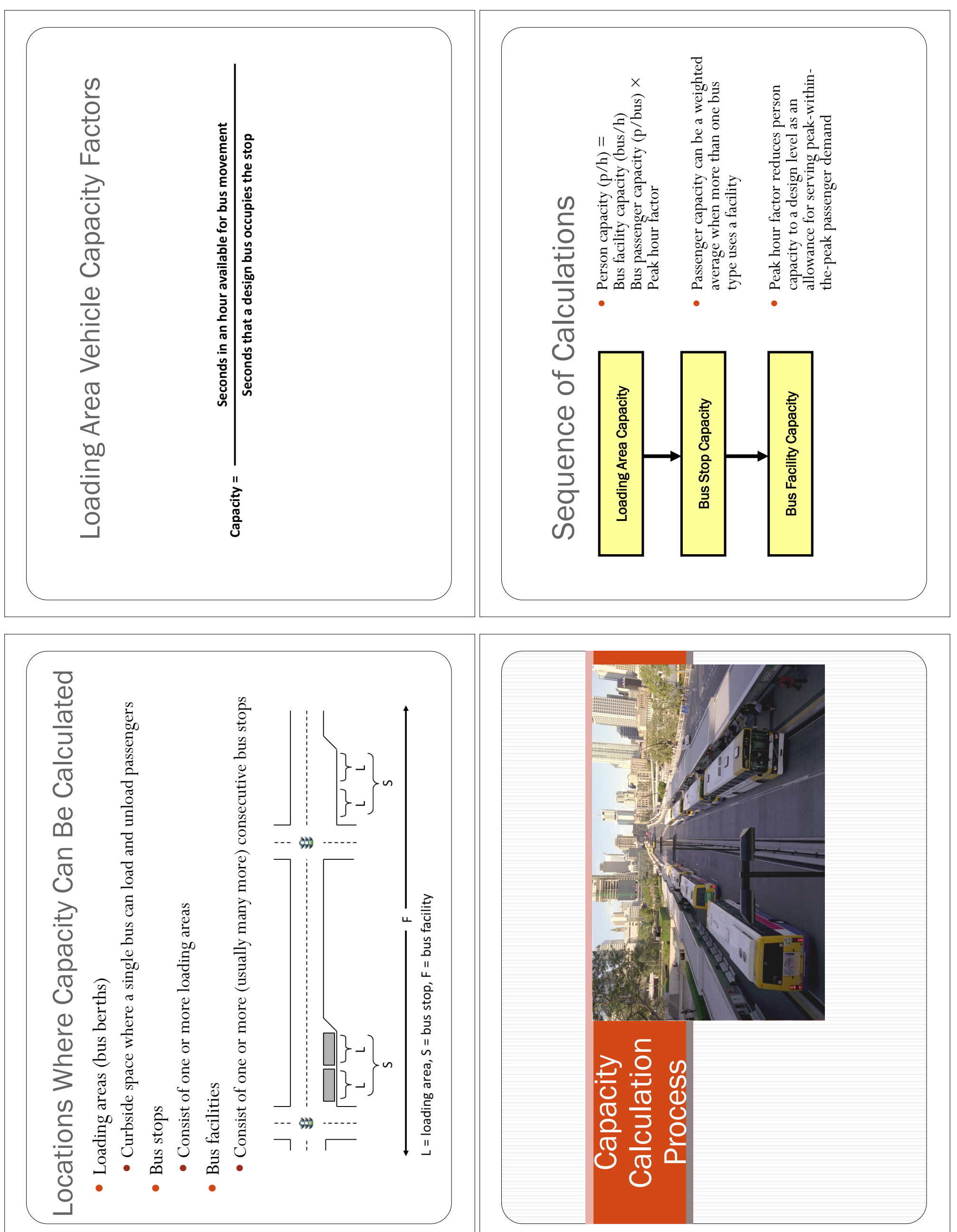

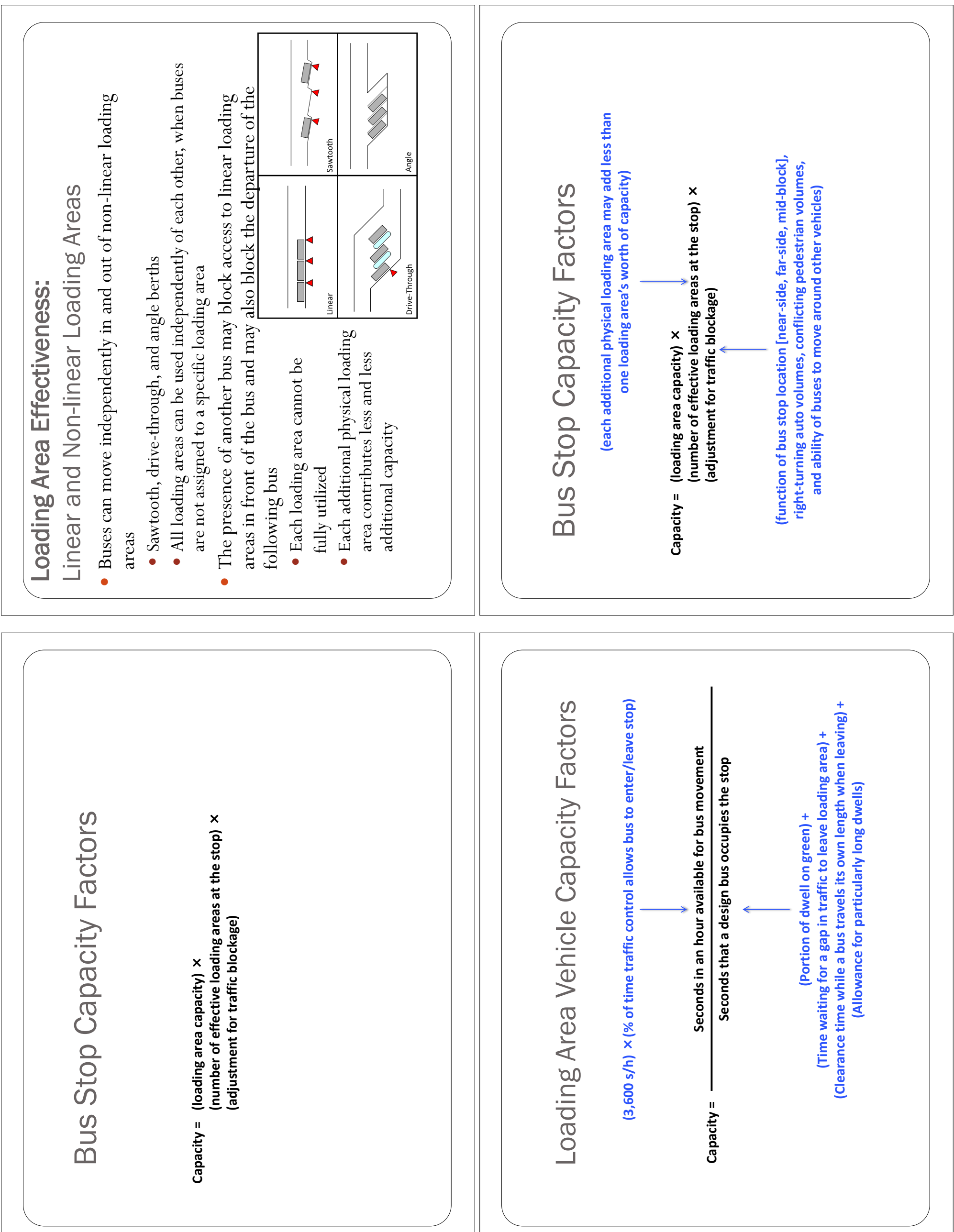

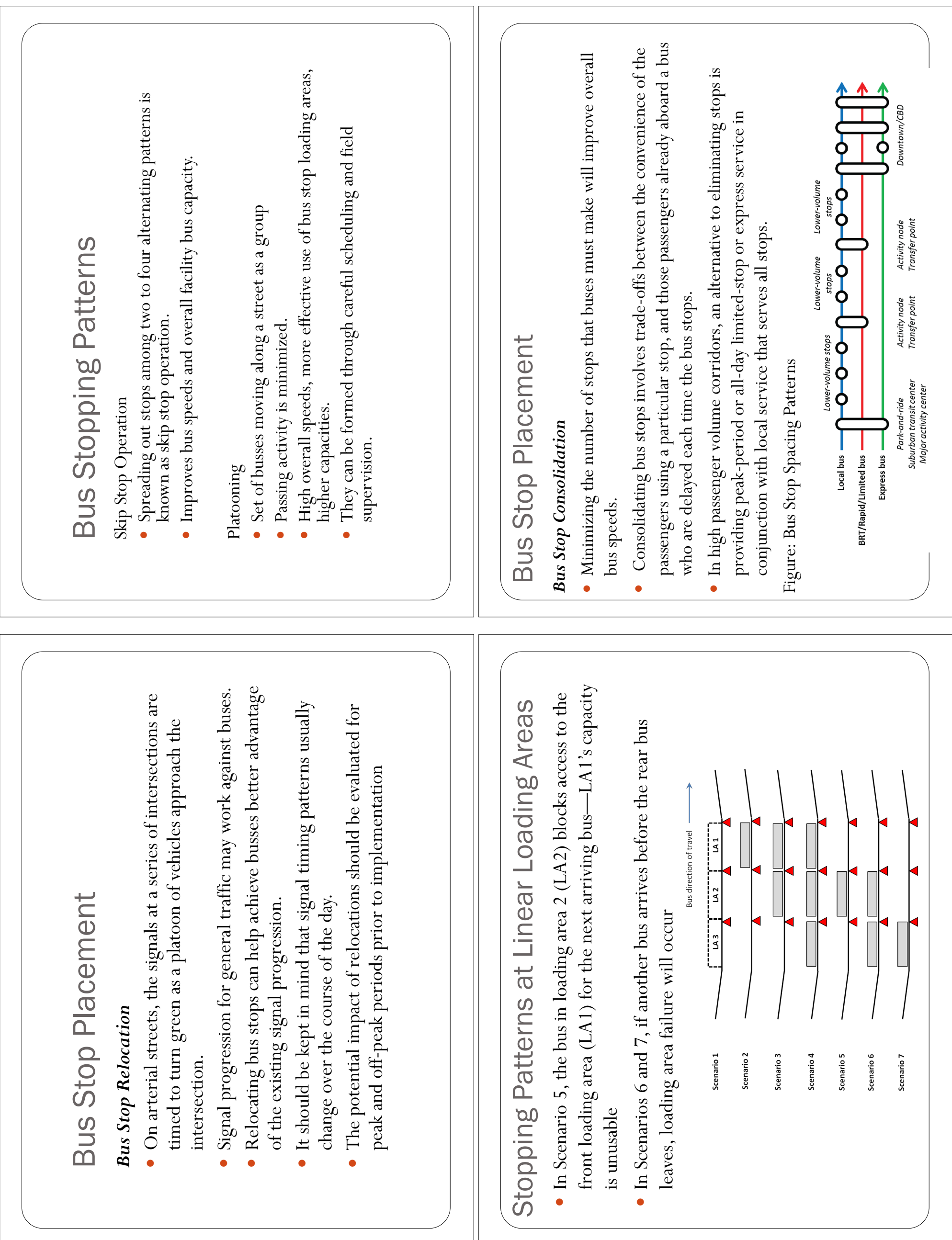


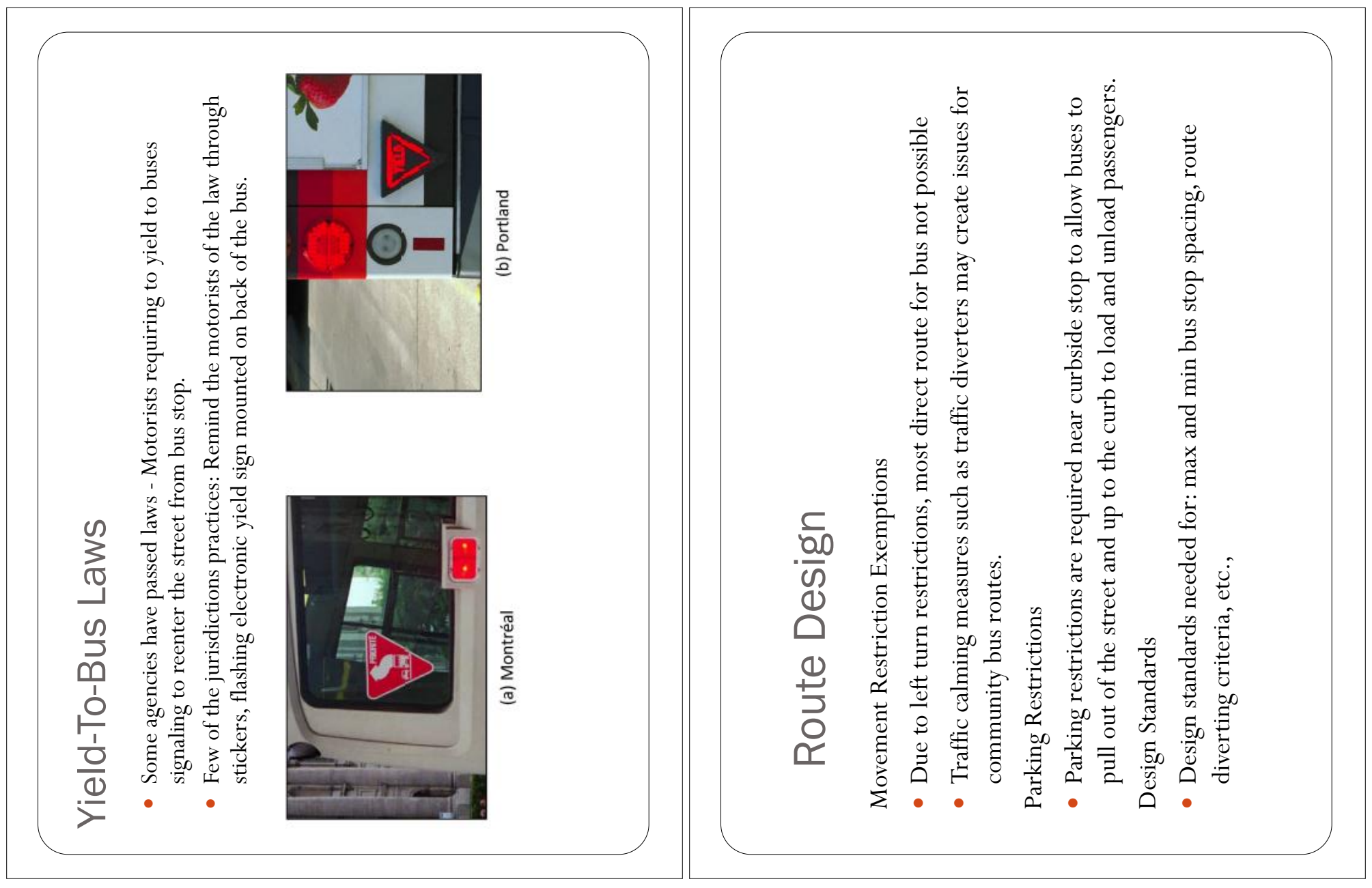



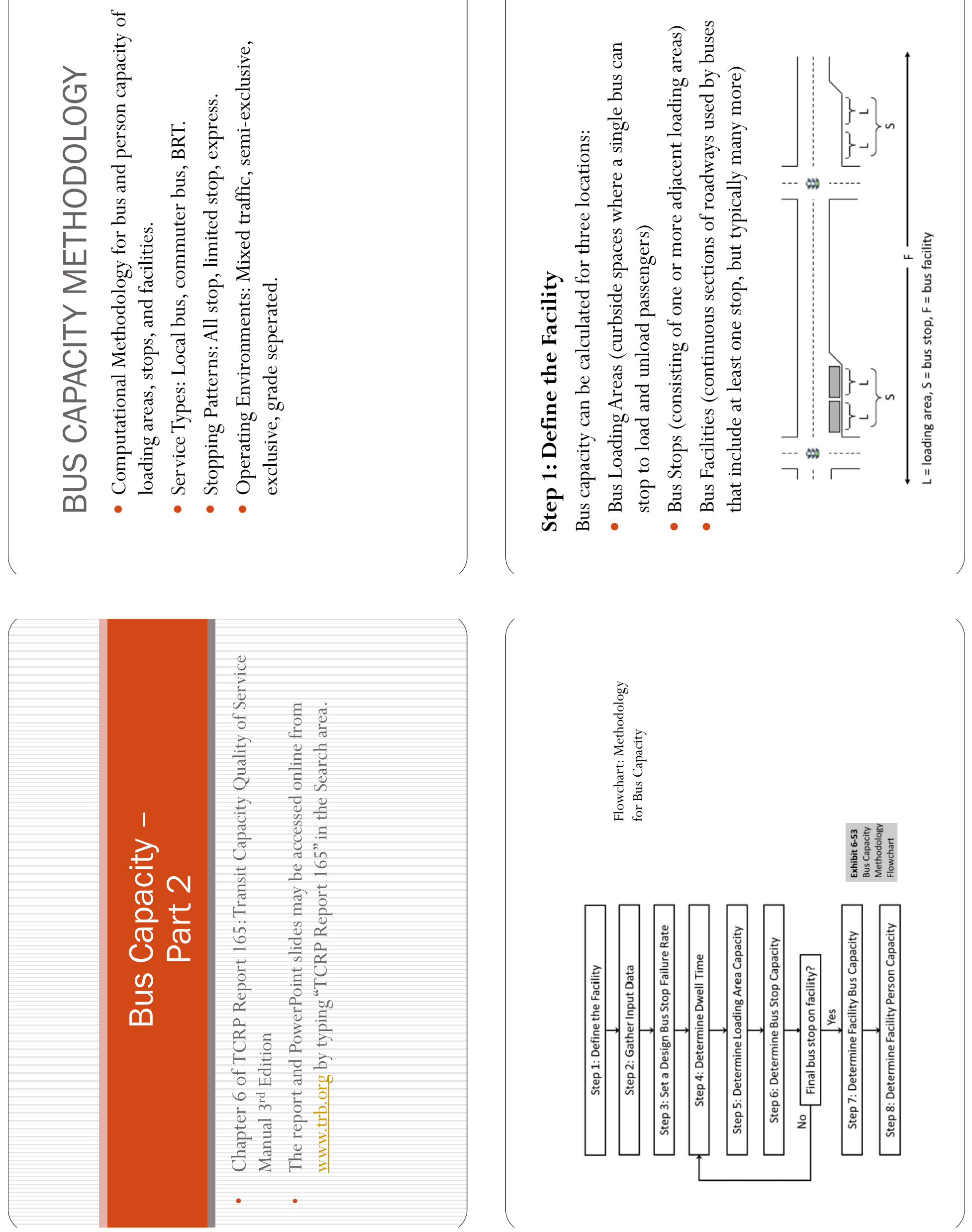


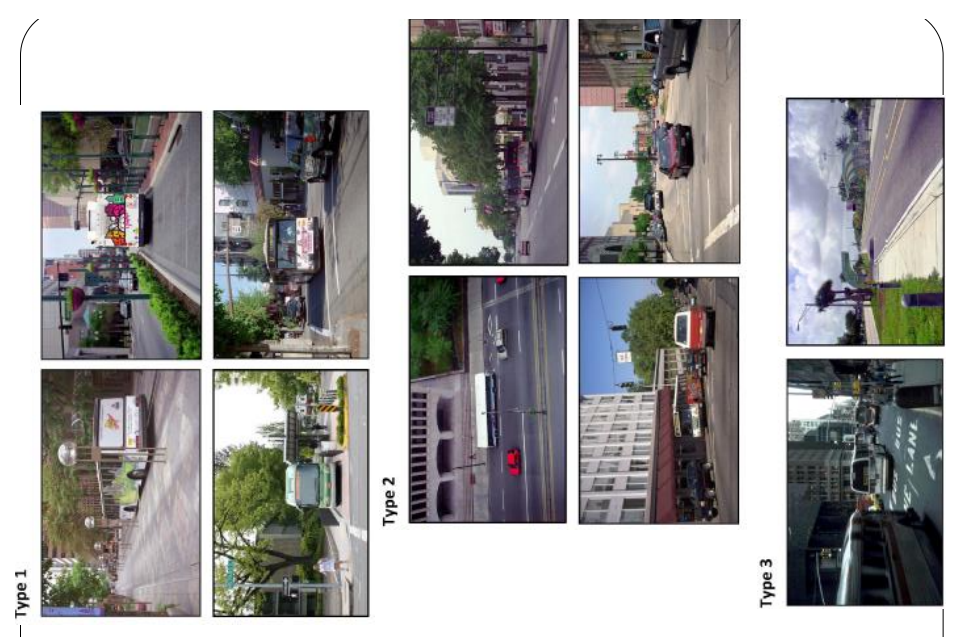

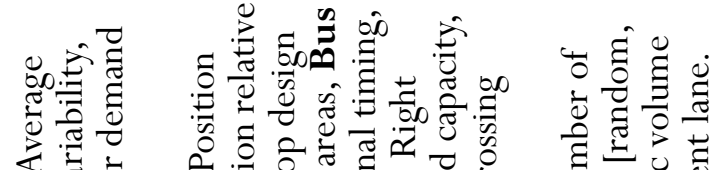

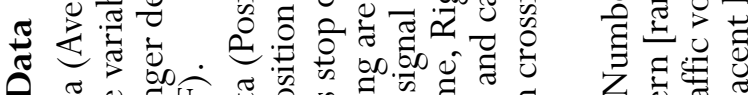

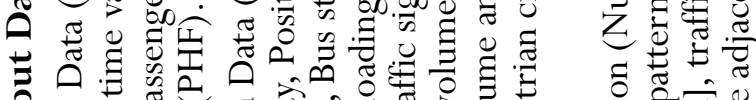

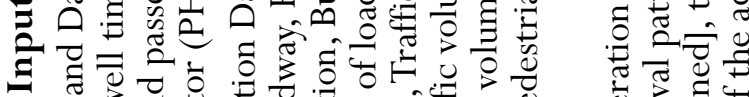
$\Xi$ ฮี

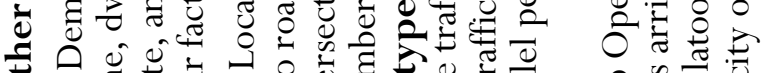

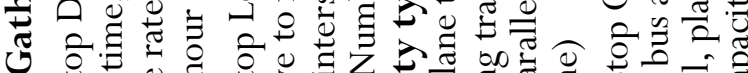

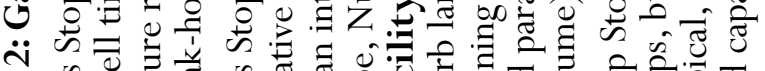

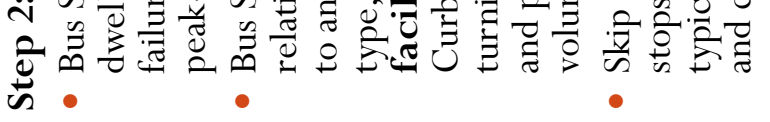

\section{.}

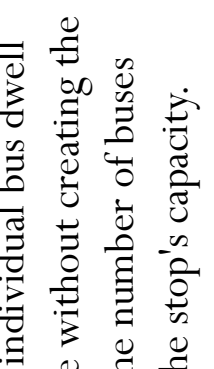

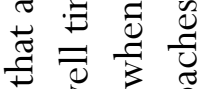

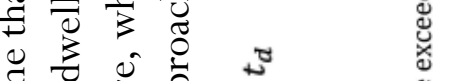

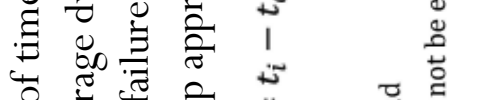

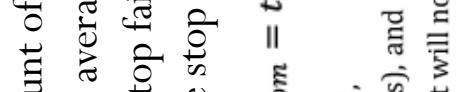

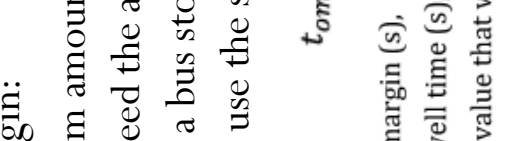
Б) छ ర

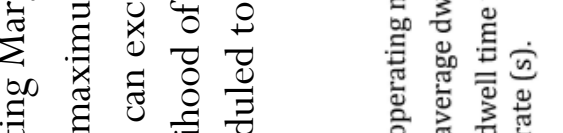

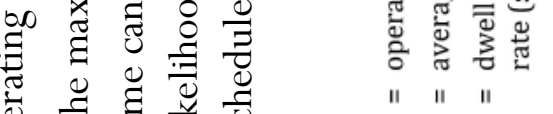

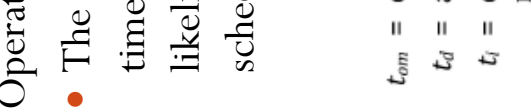
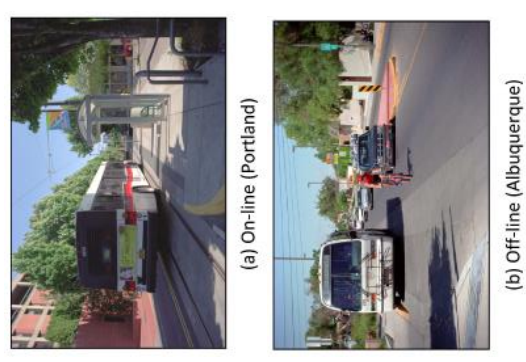

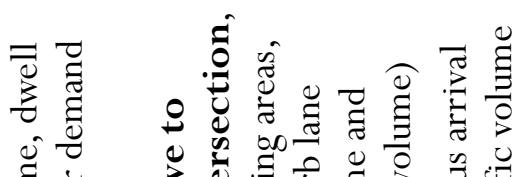

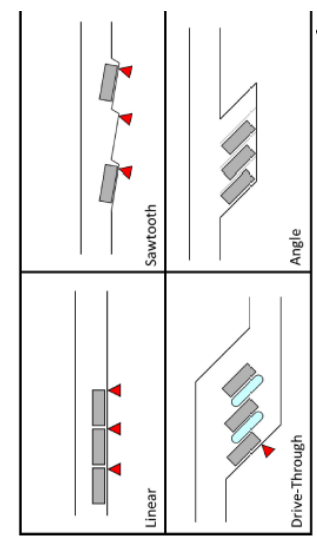

छ

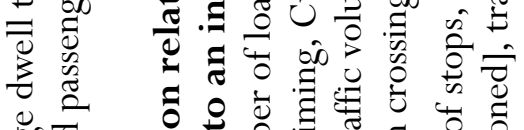

क्ञ

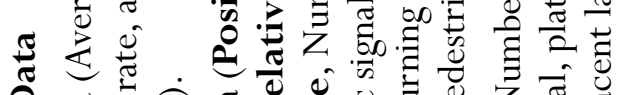

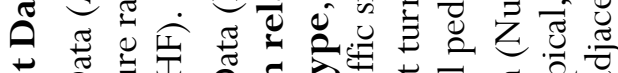

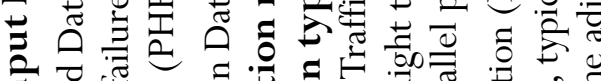

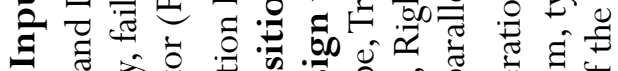

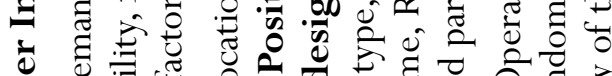

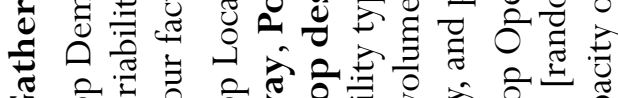

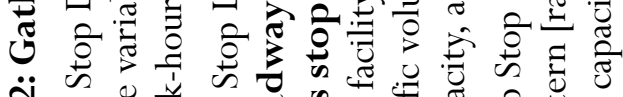

讨

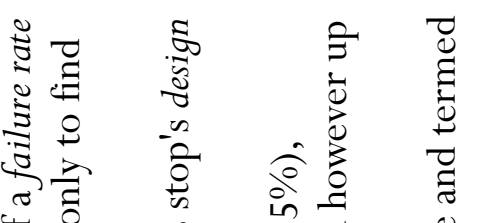

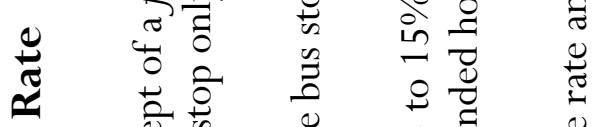

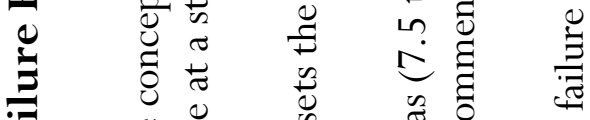

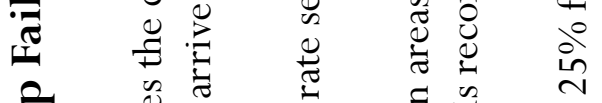

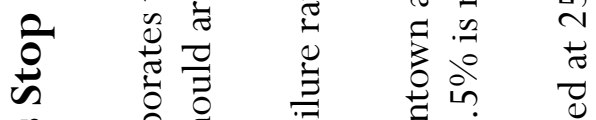

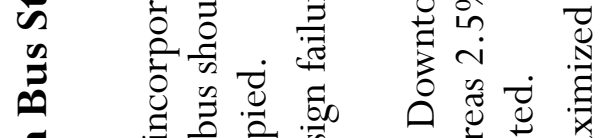

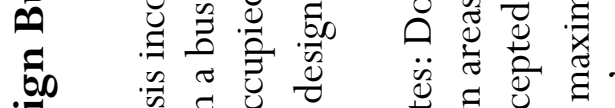

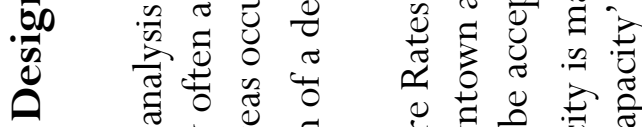

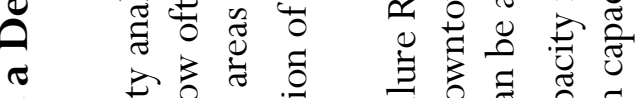

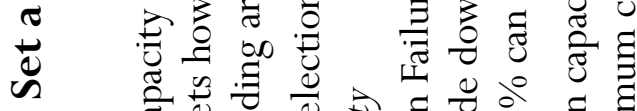

$\ddot{n}$ चि

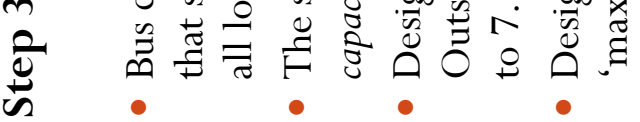



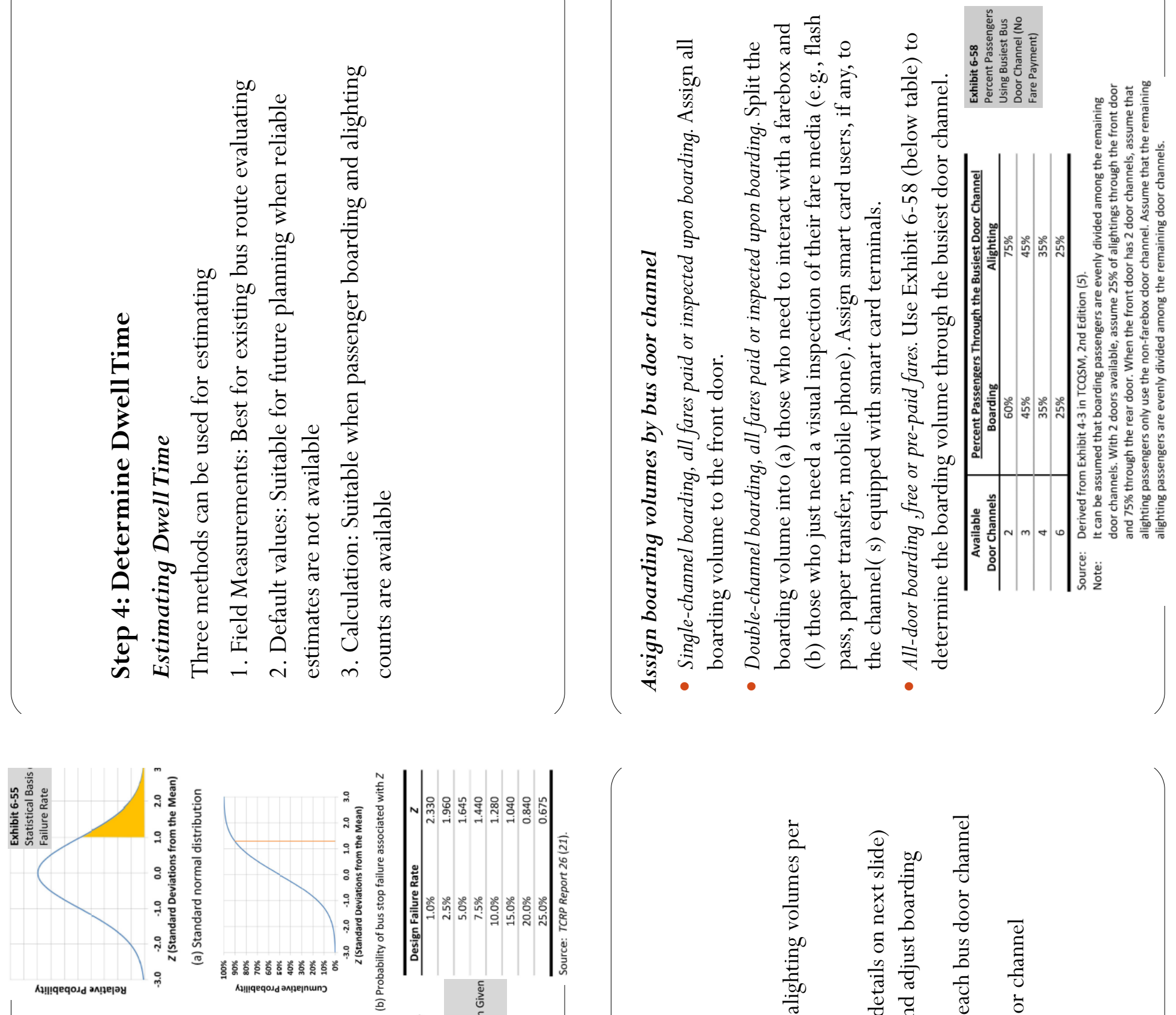

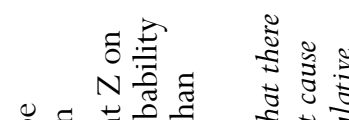

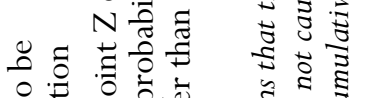

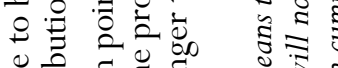

๑

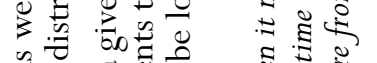

ป

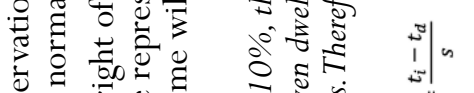

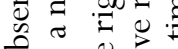

요욜

完 8 :

渮

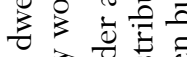

的高

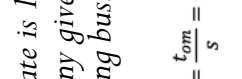

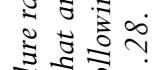

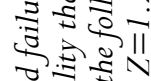

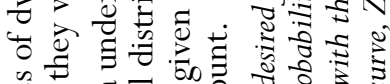

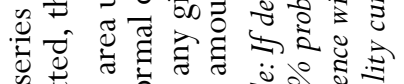

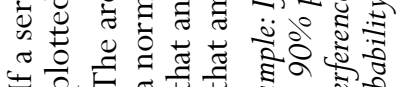

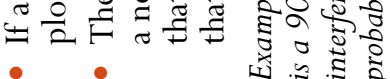

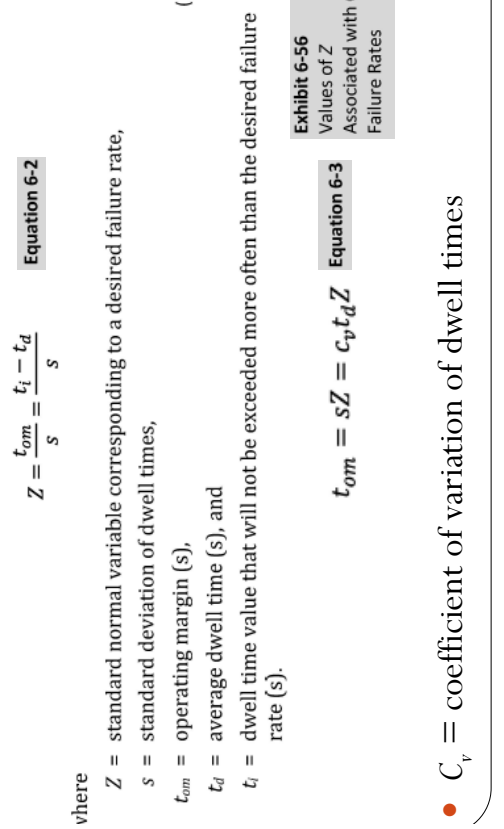

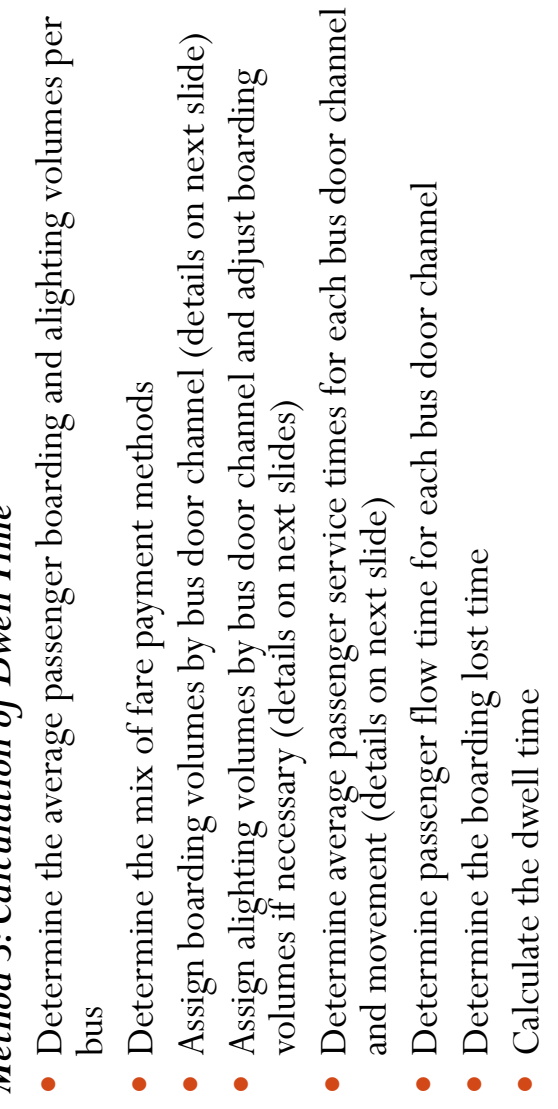



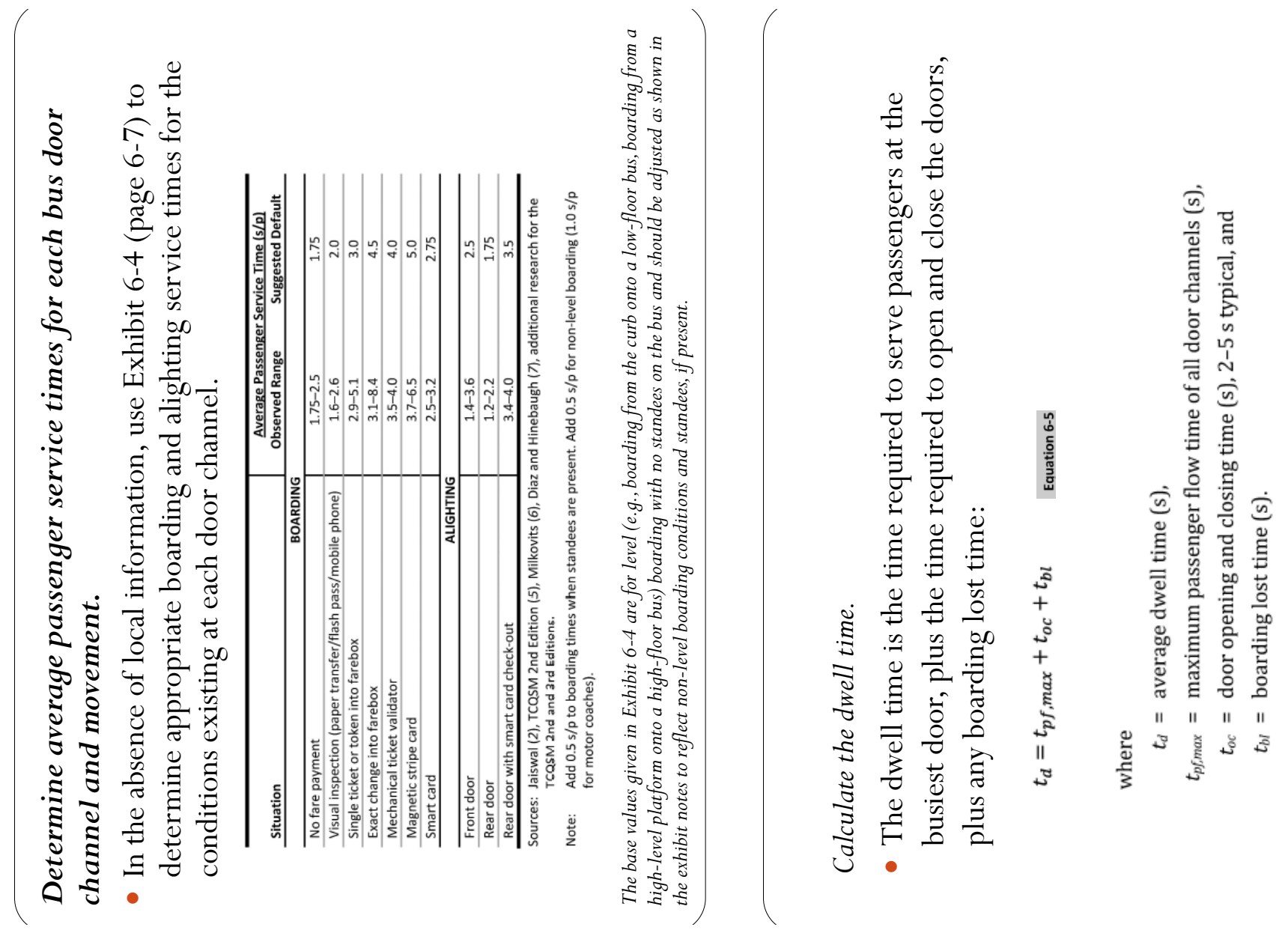

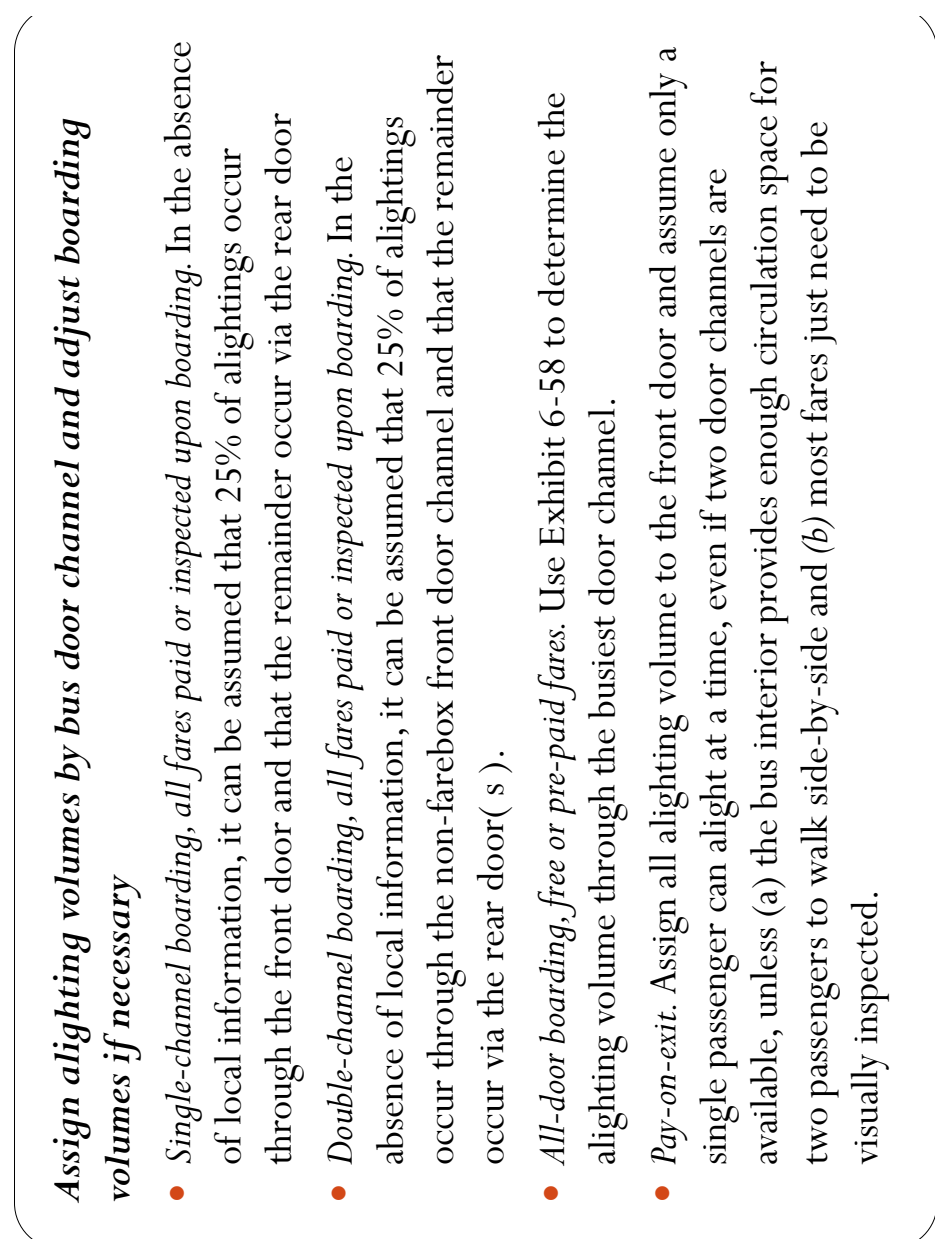

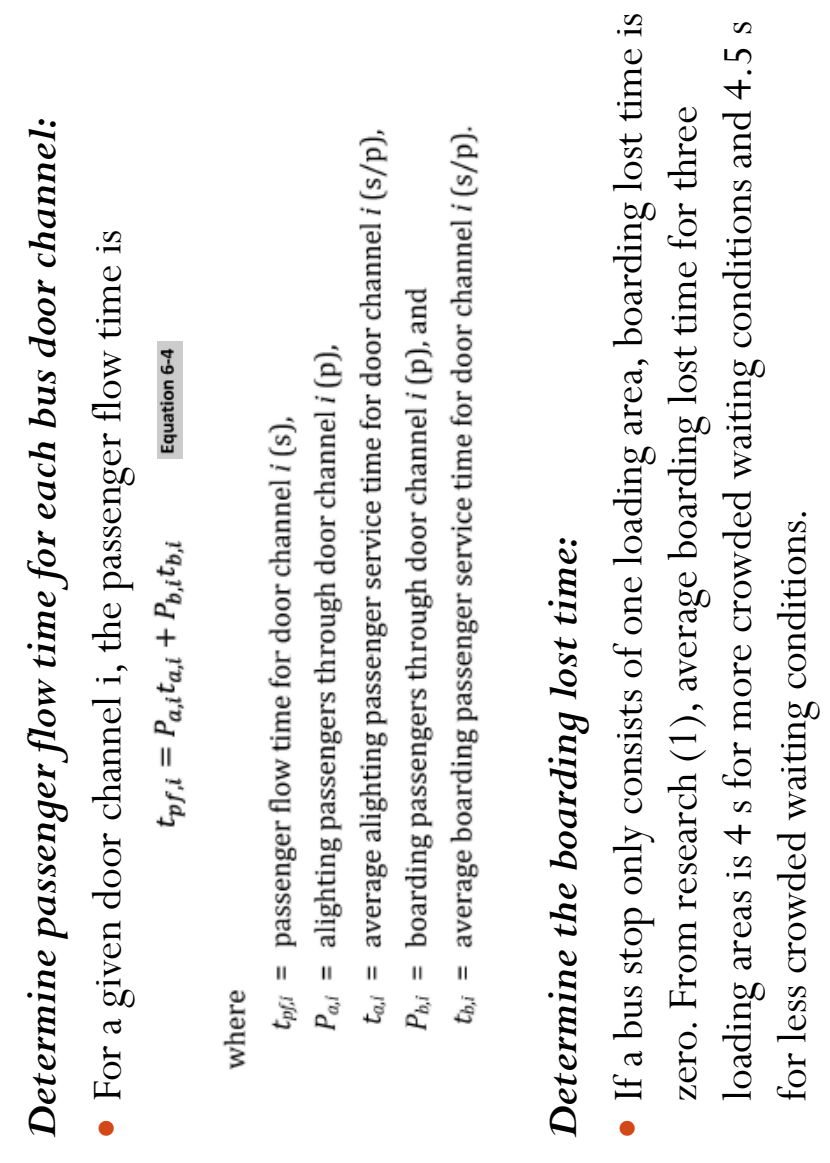



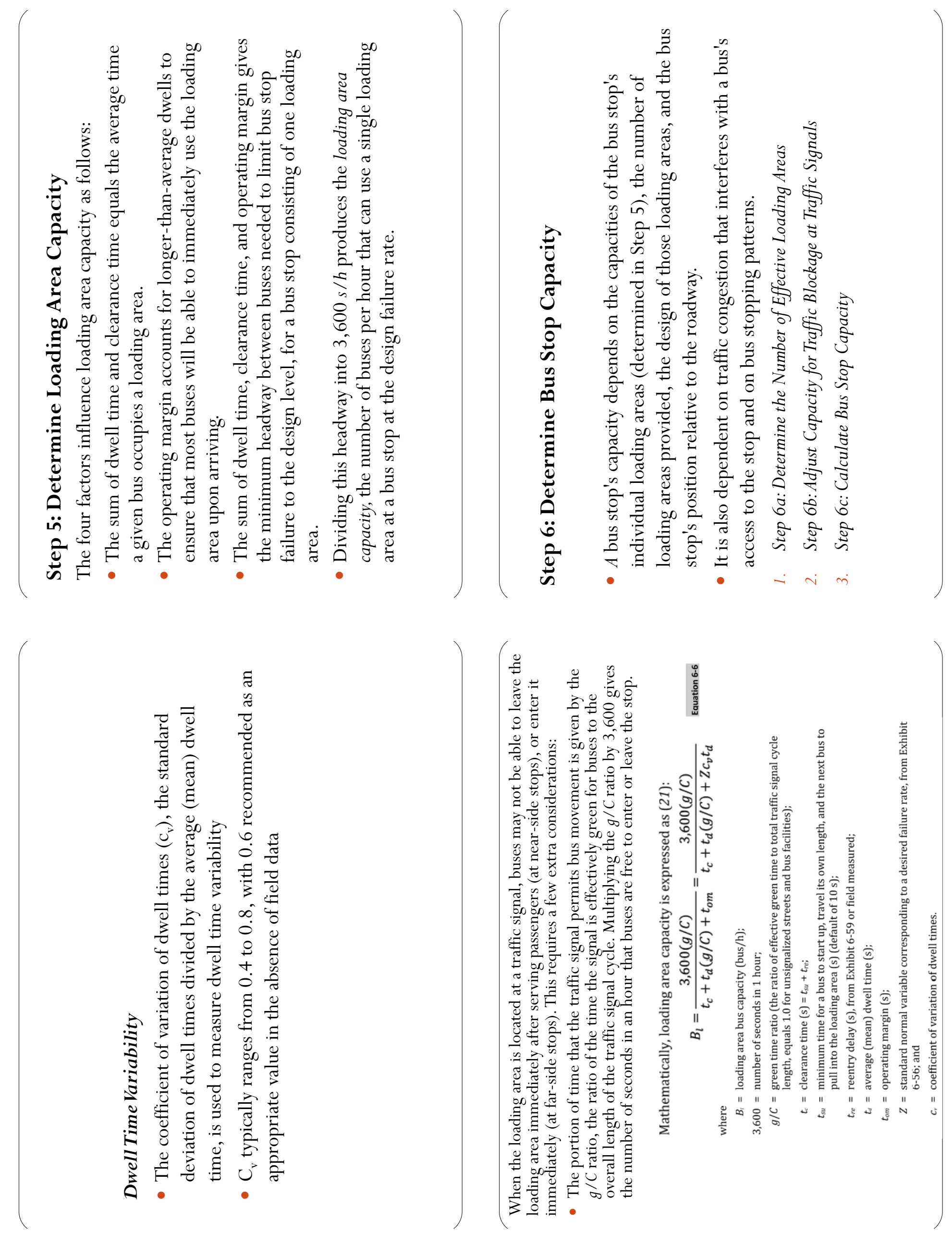

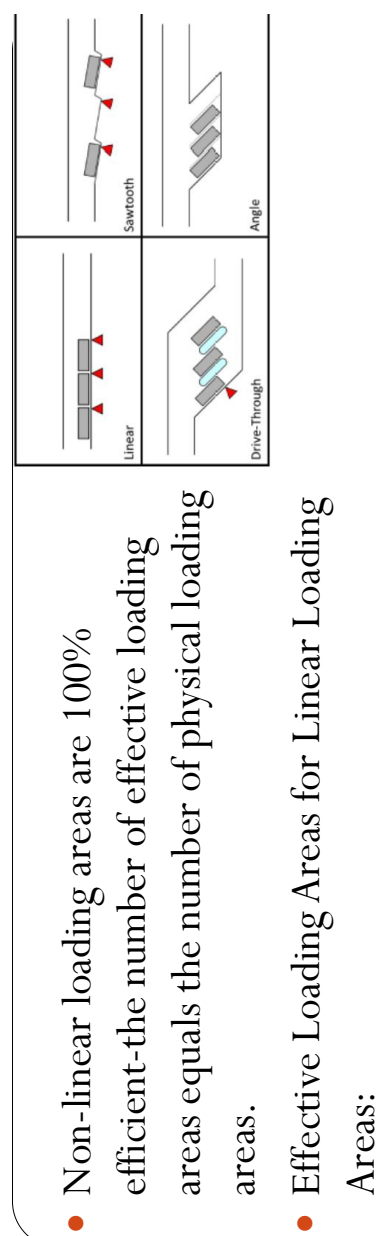
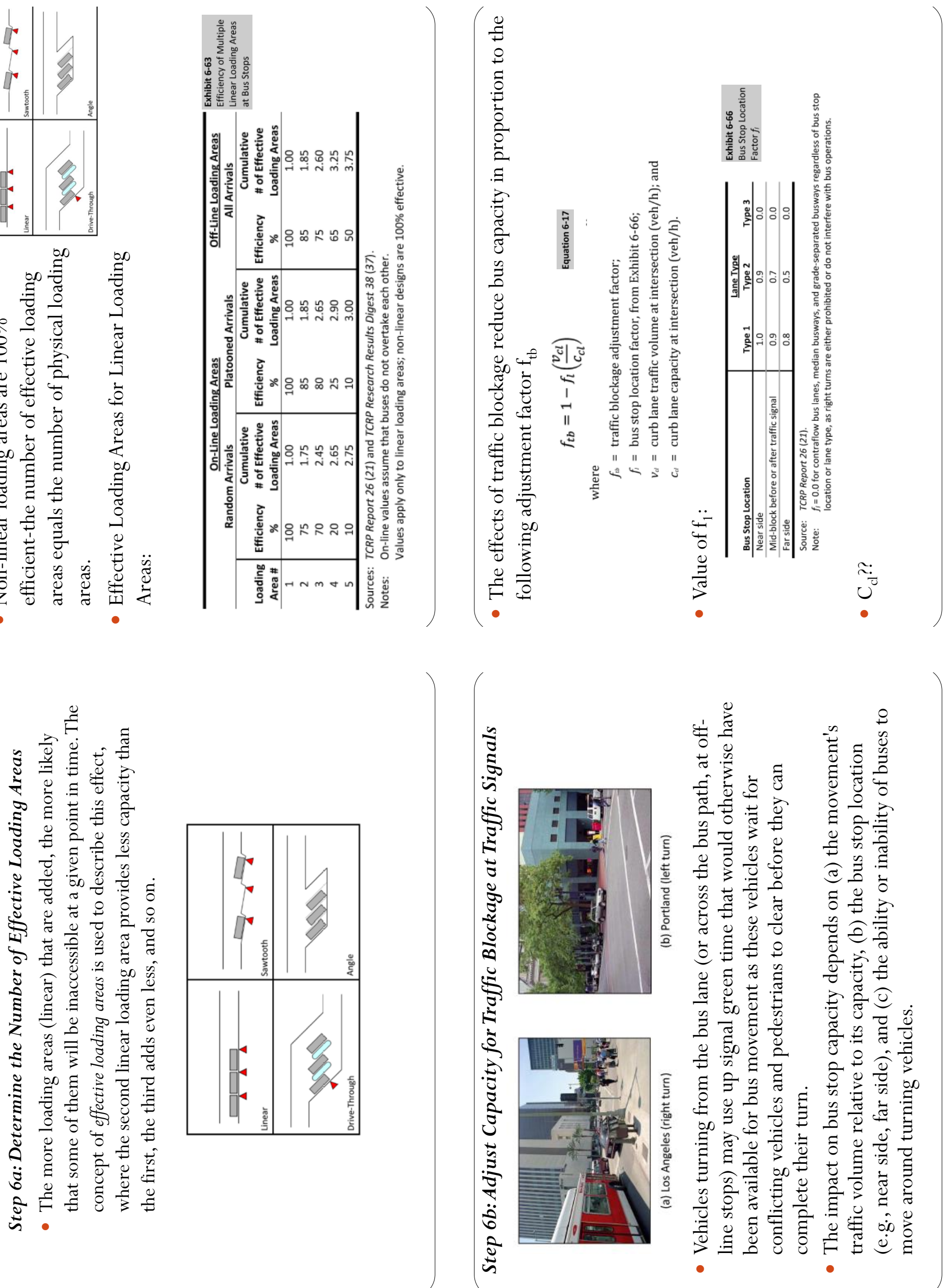

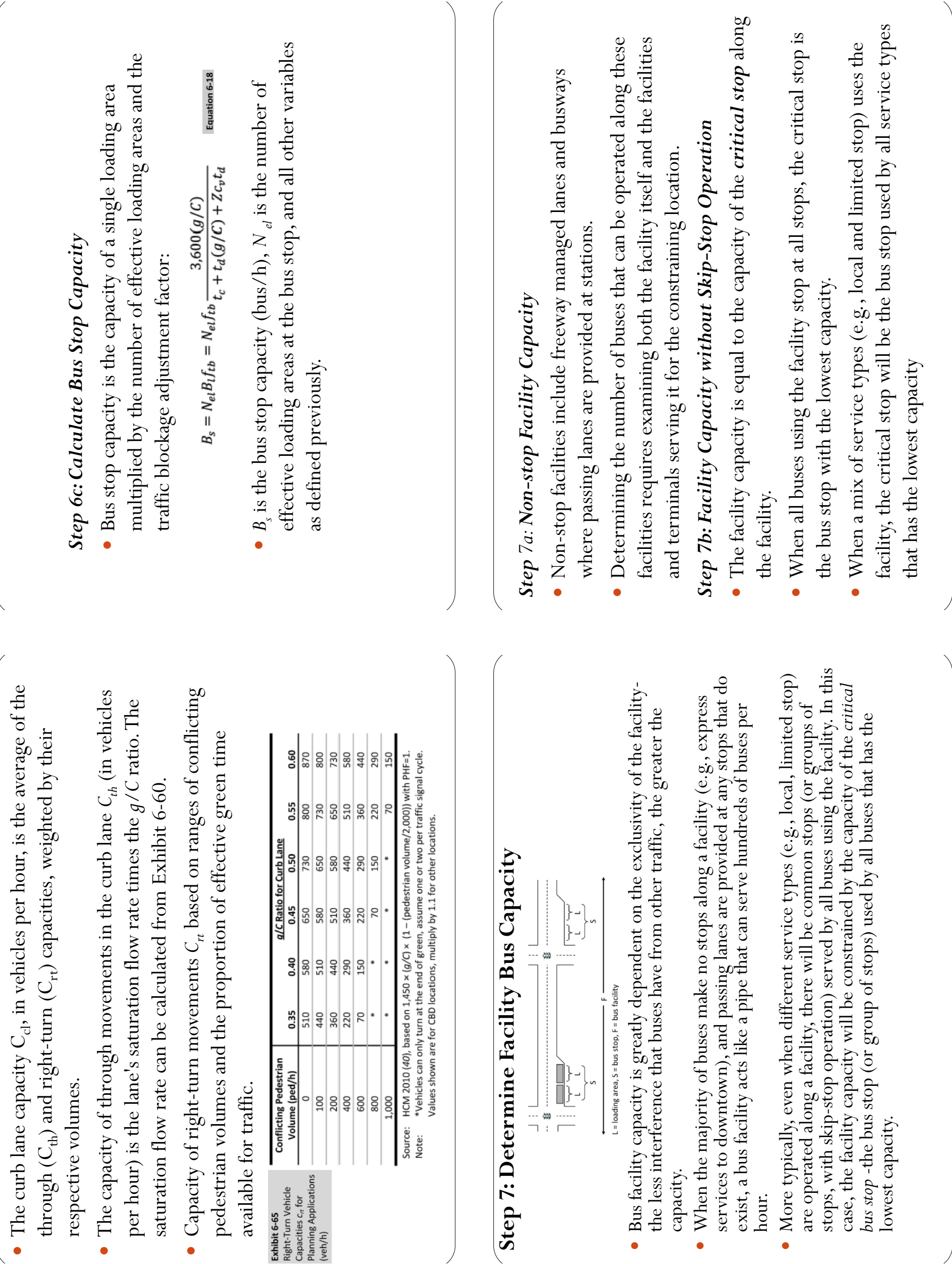

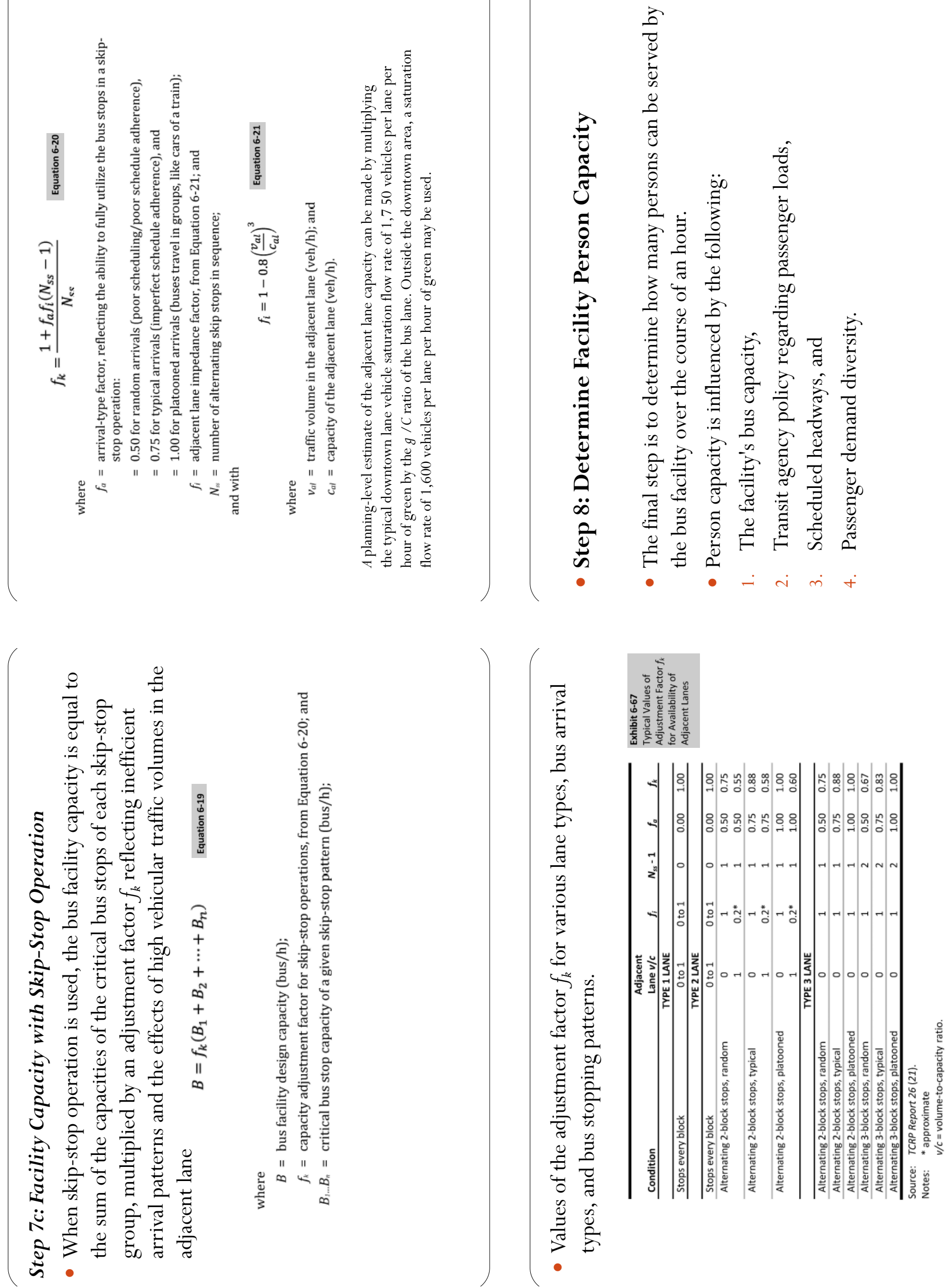

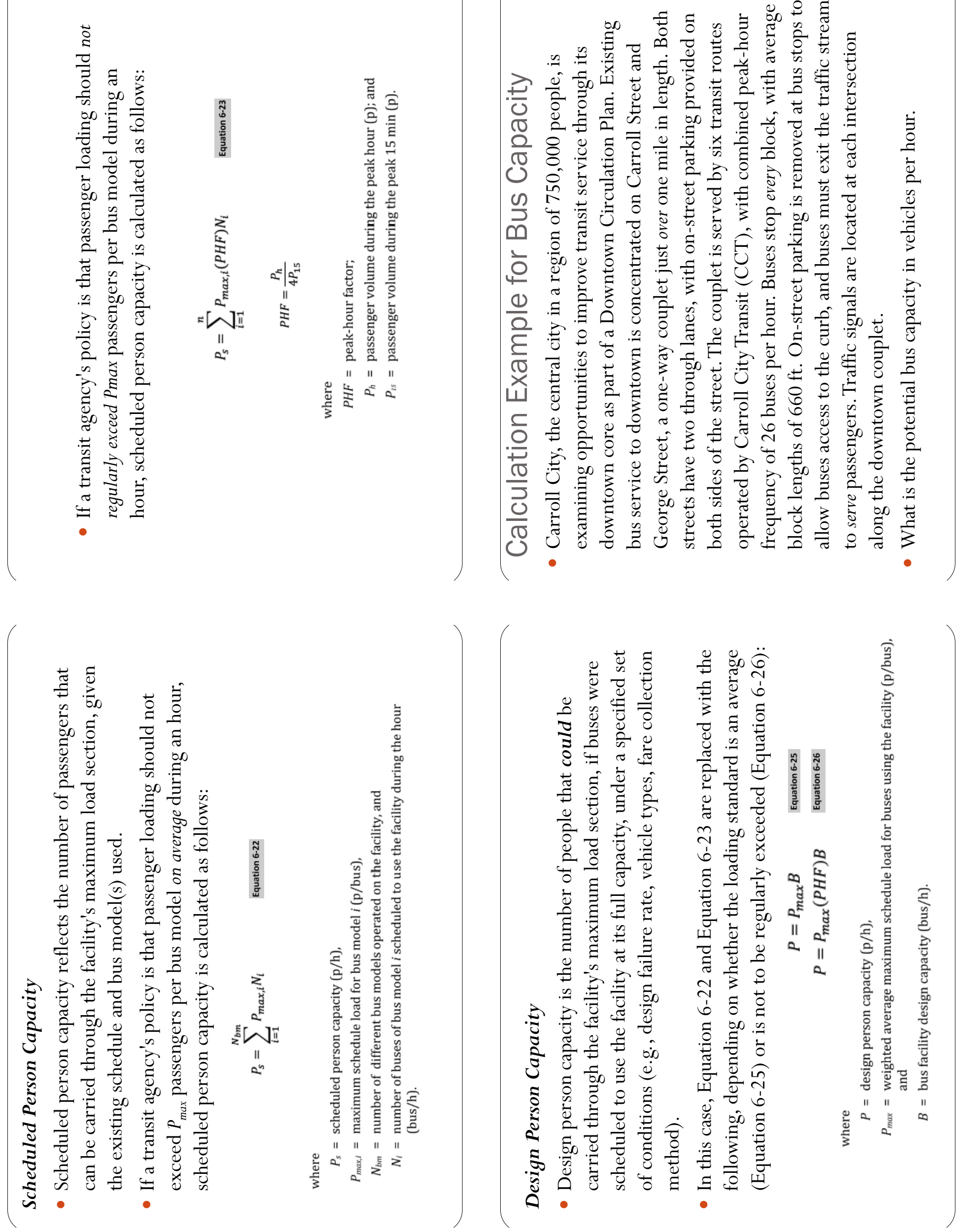

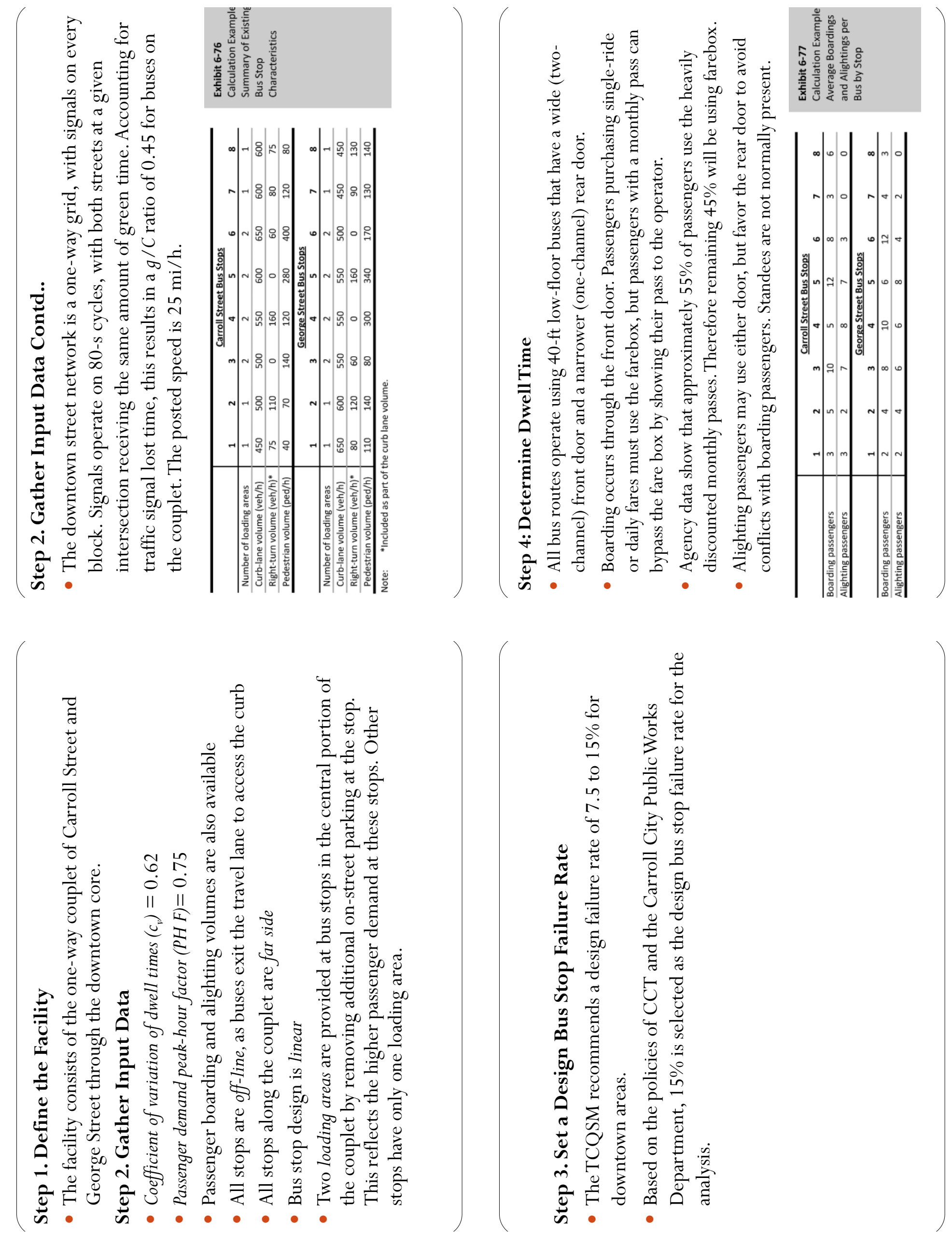

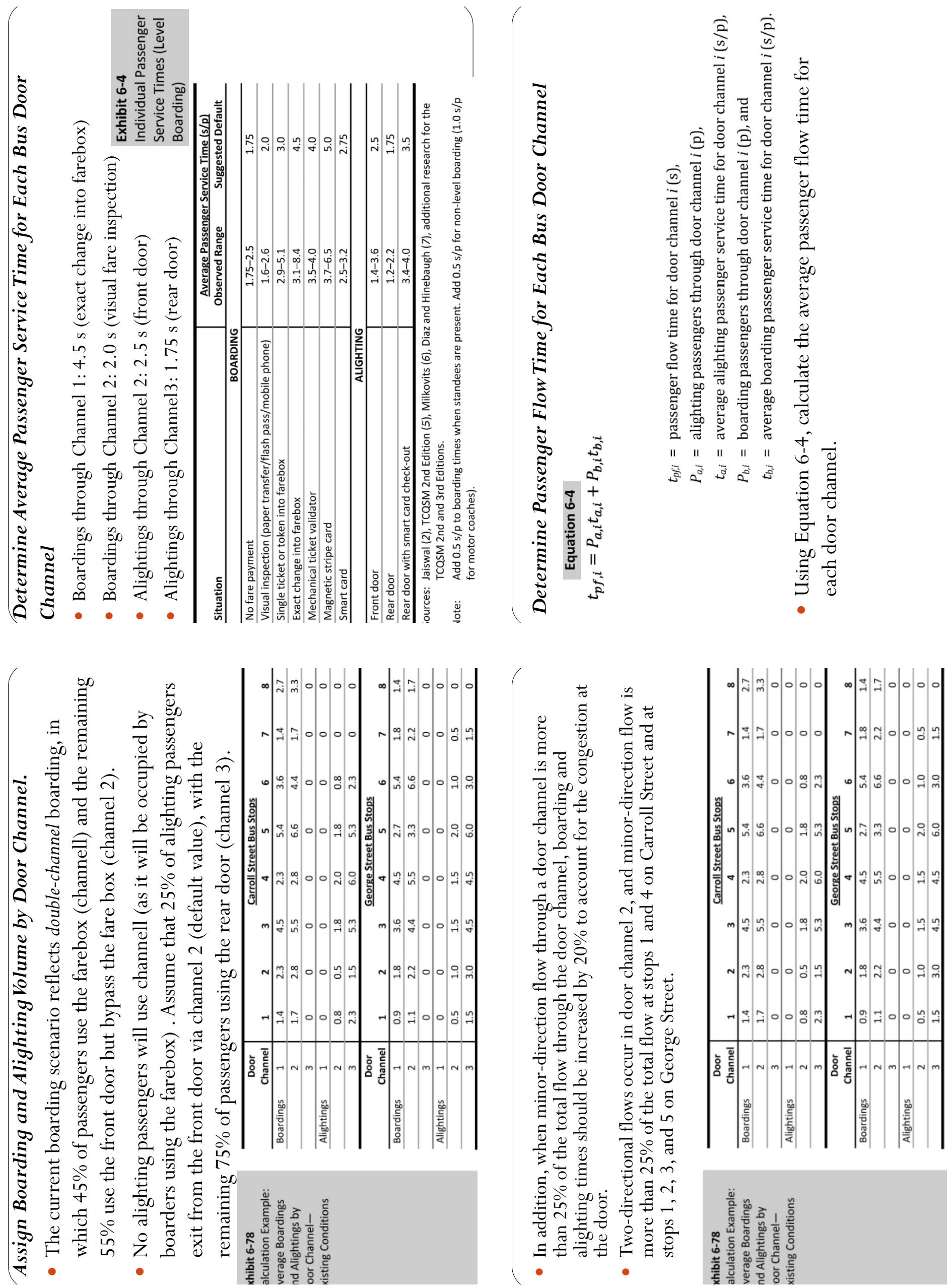

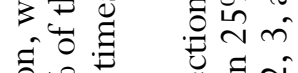

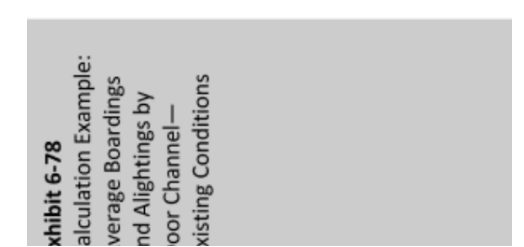



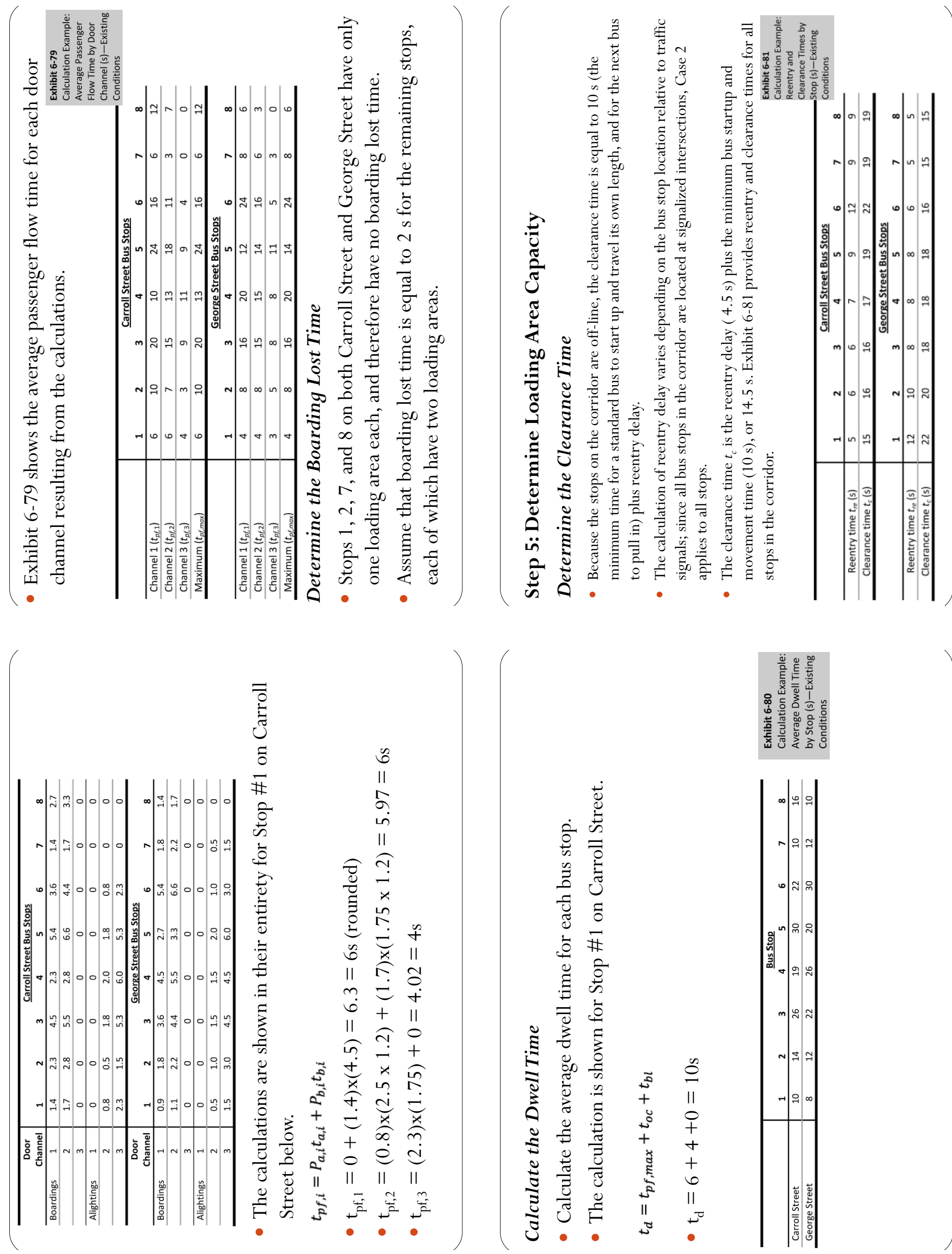

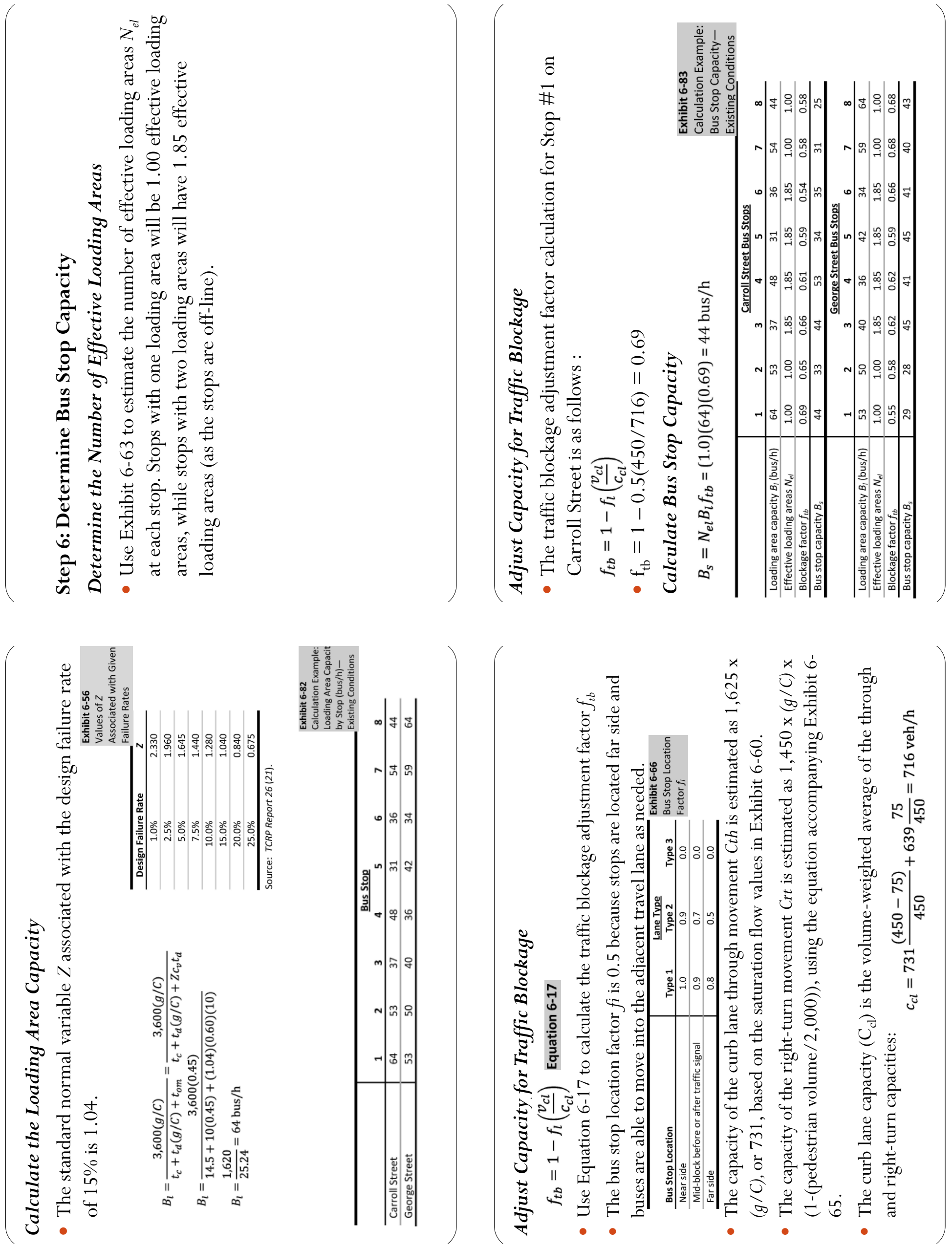

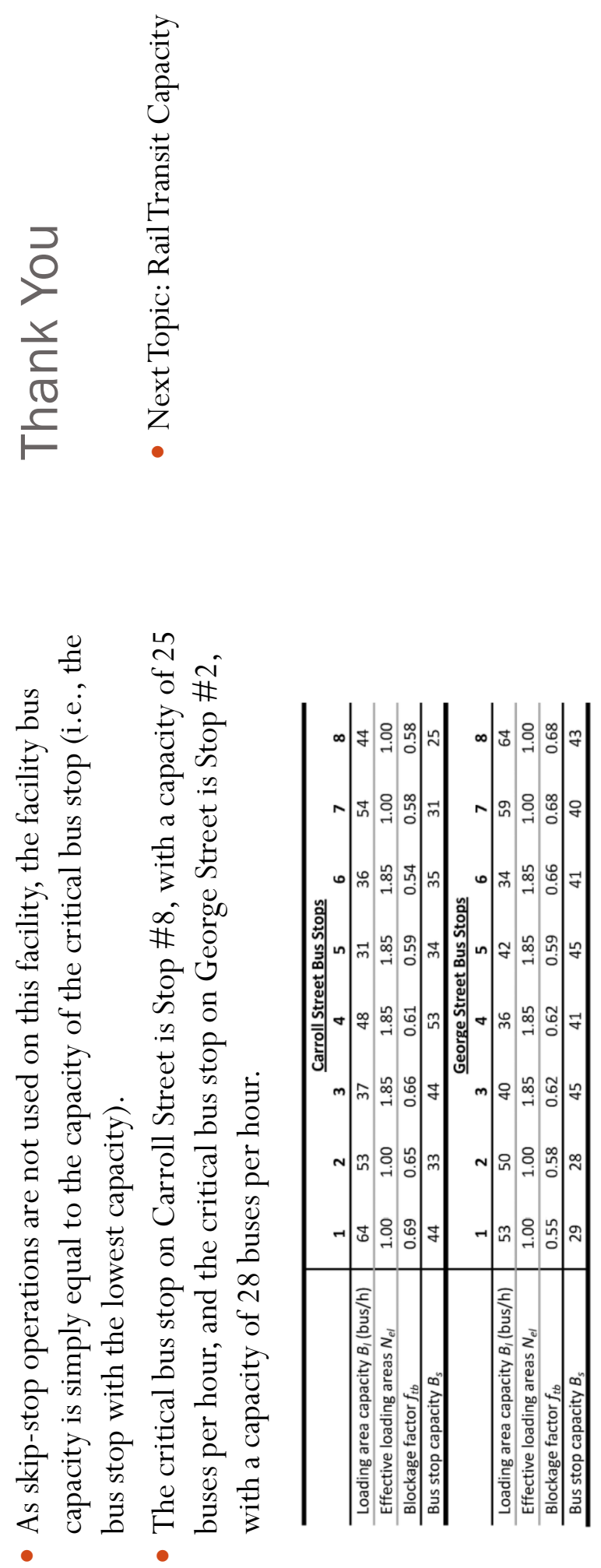

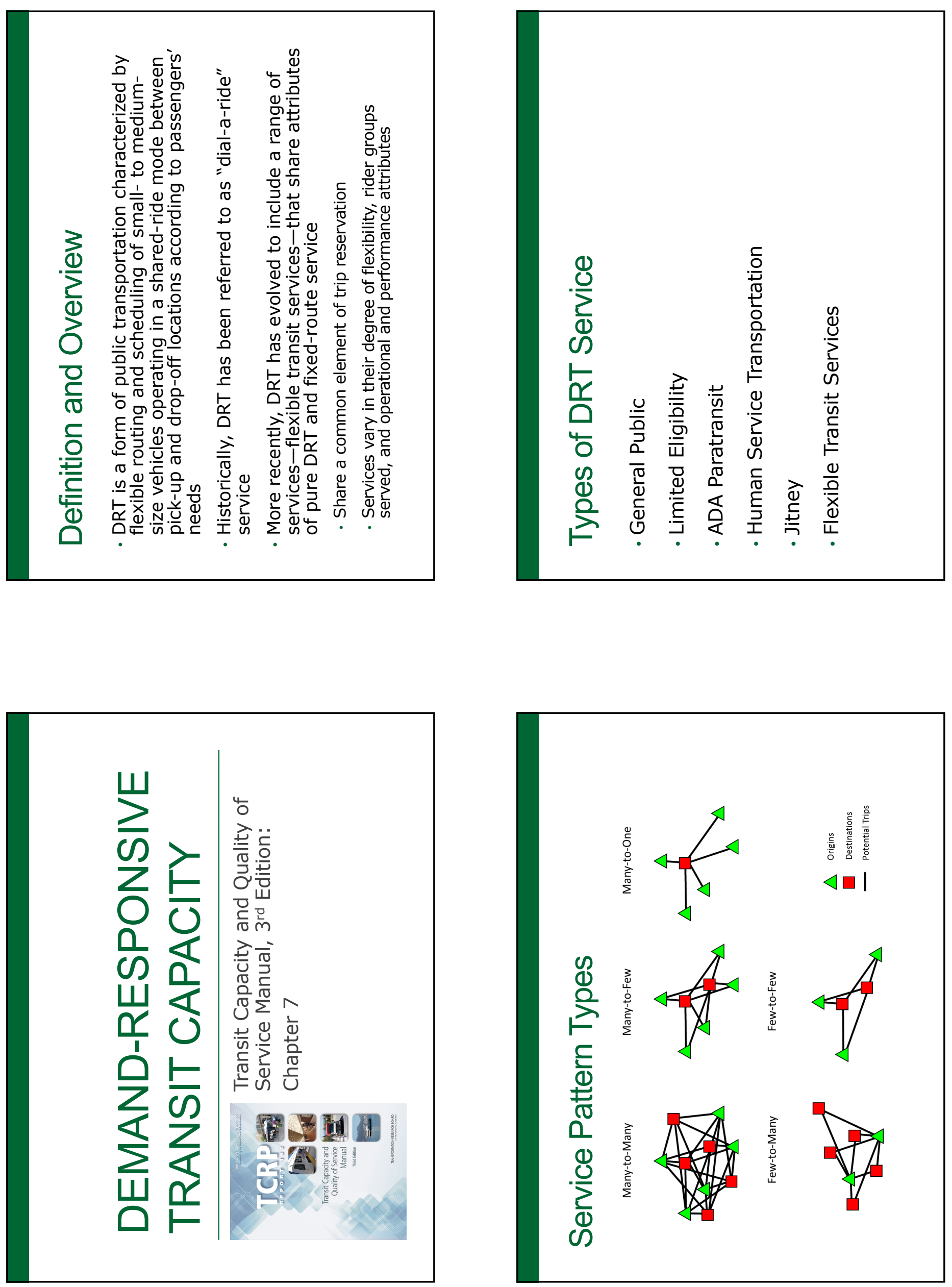

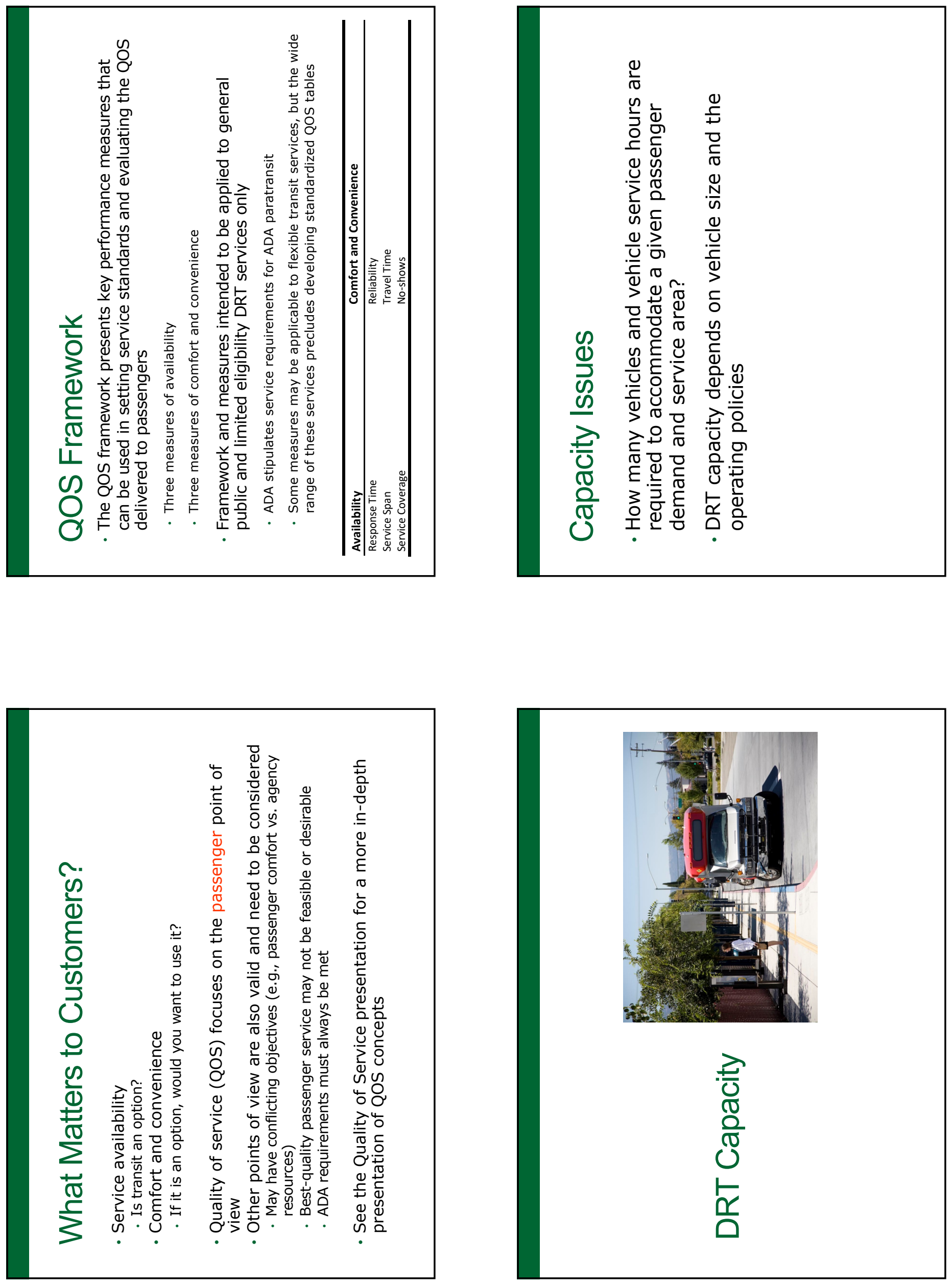

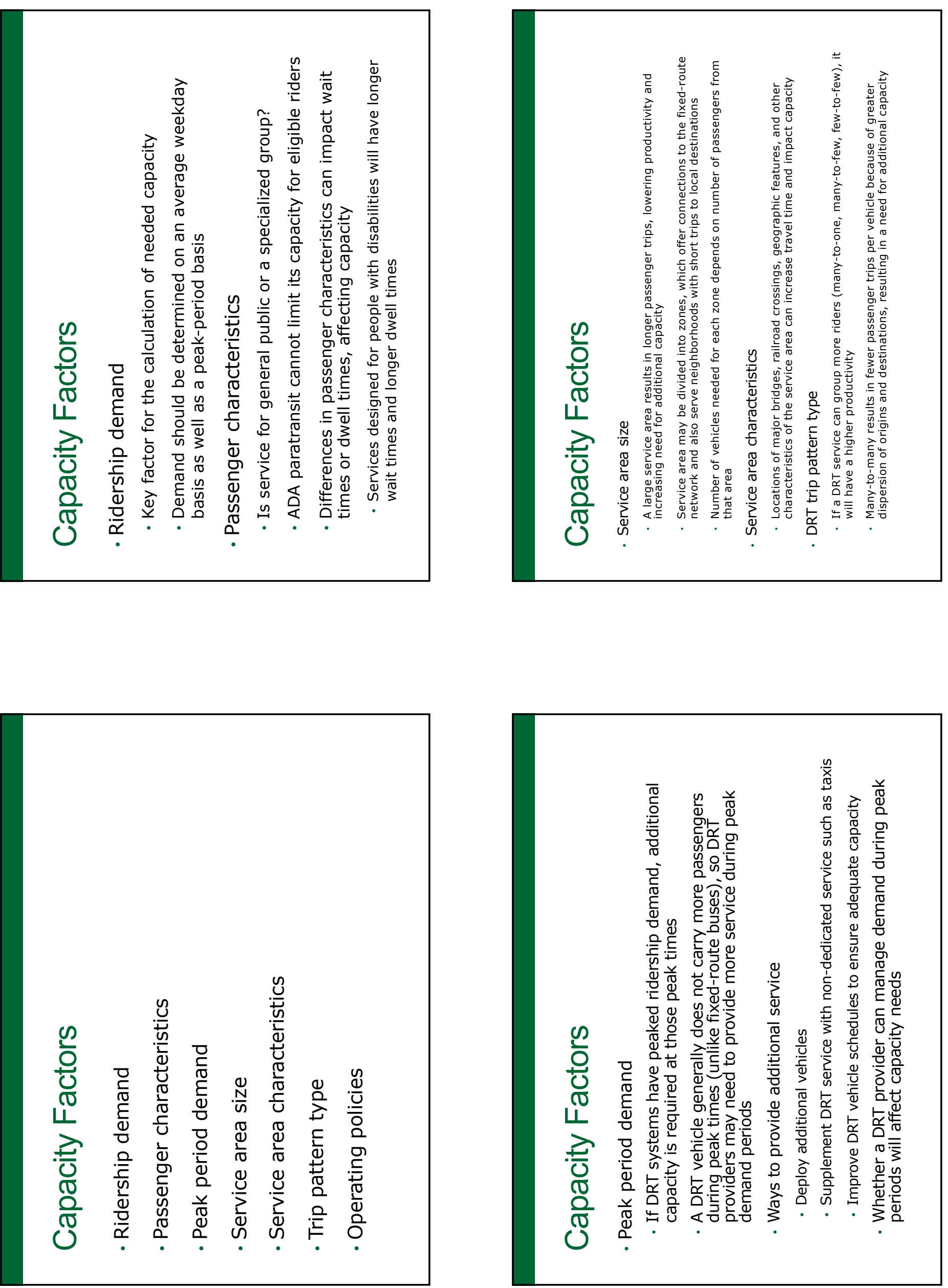

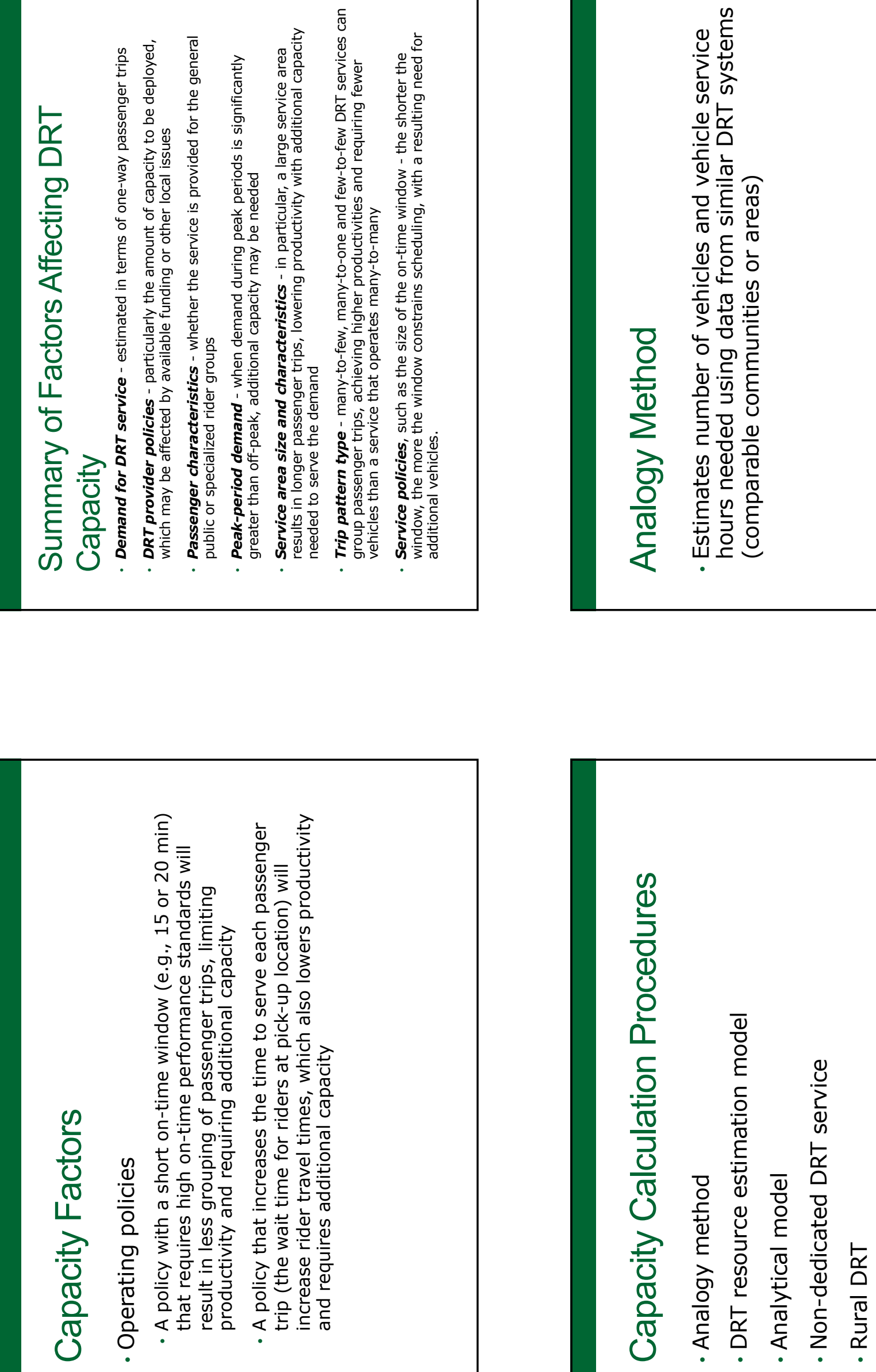

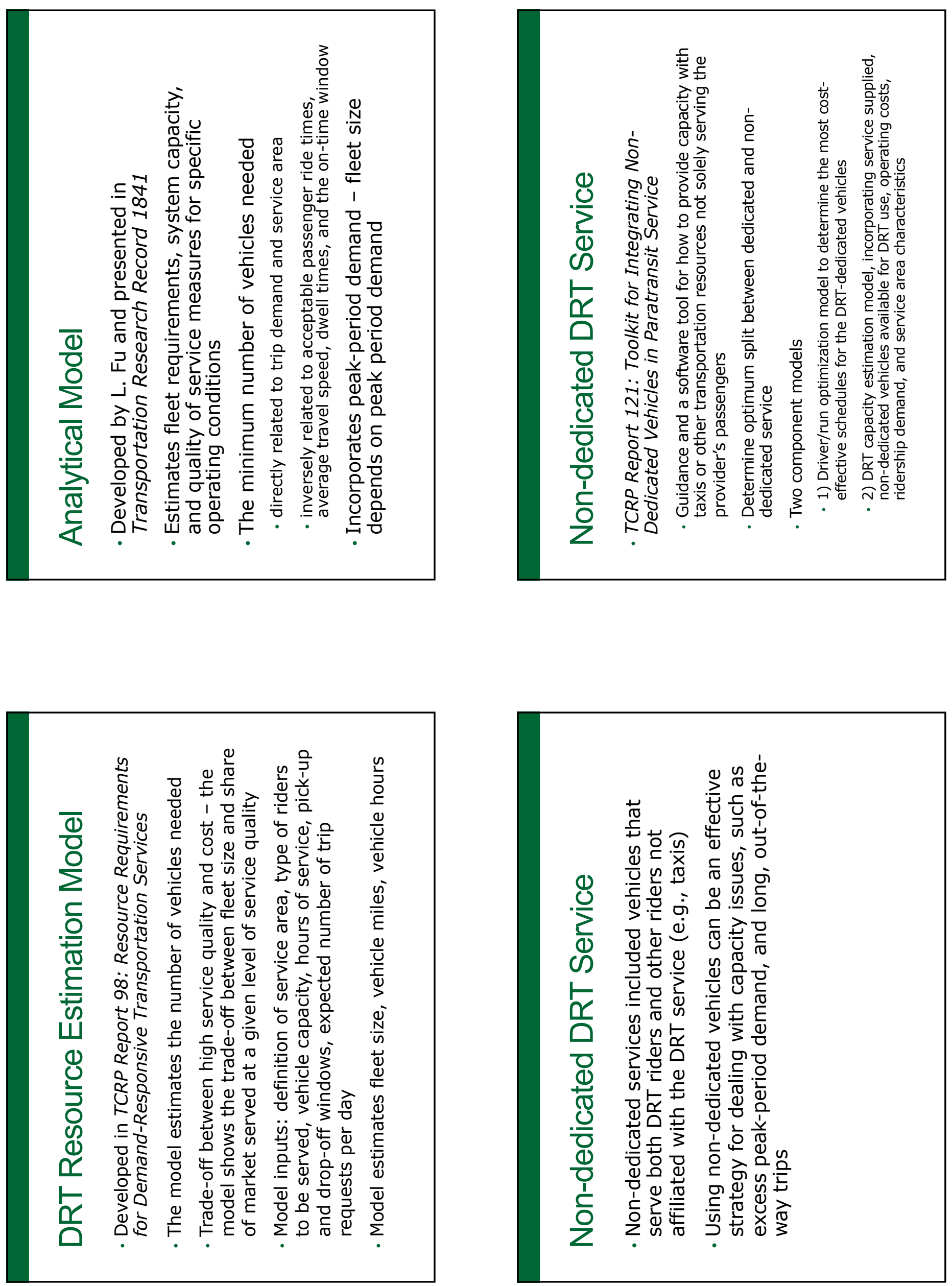

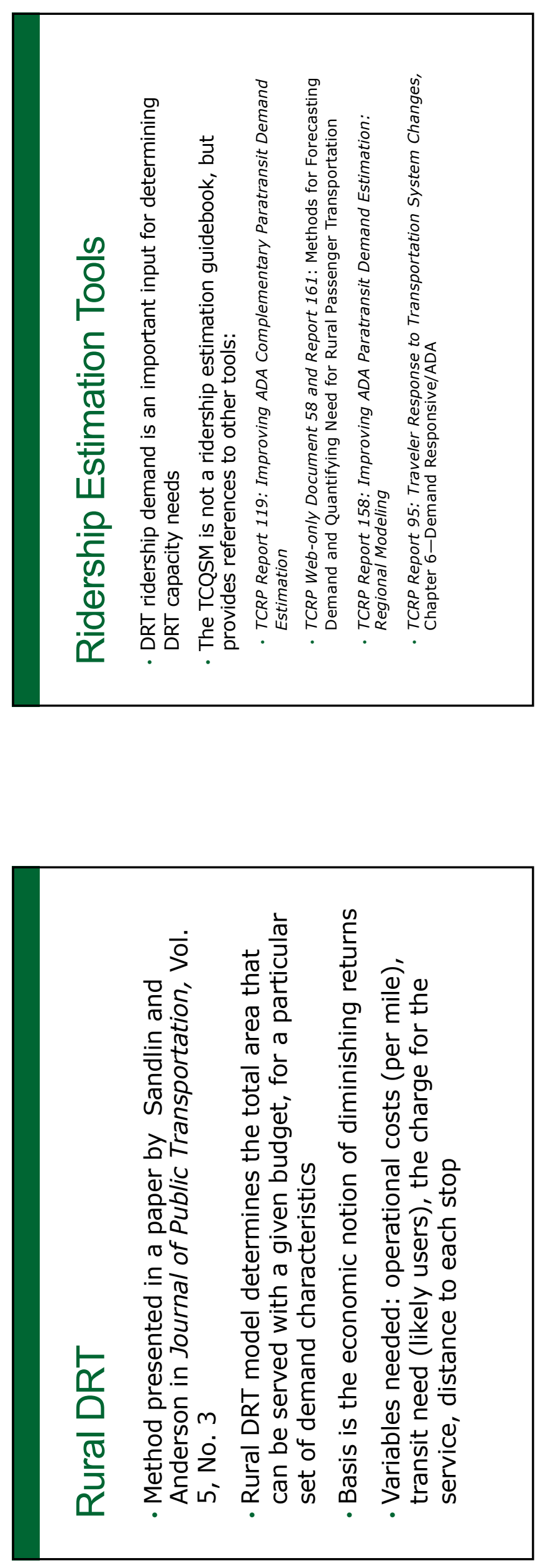

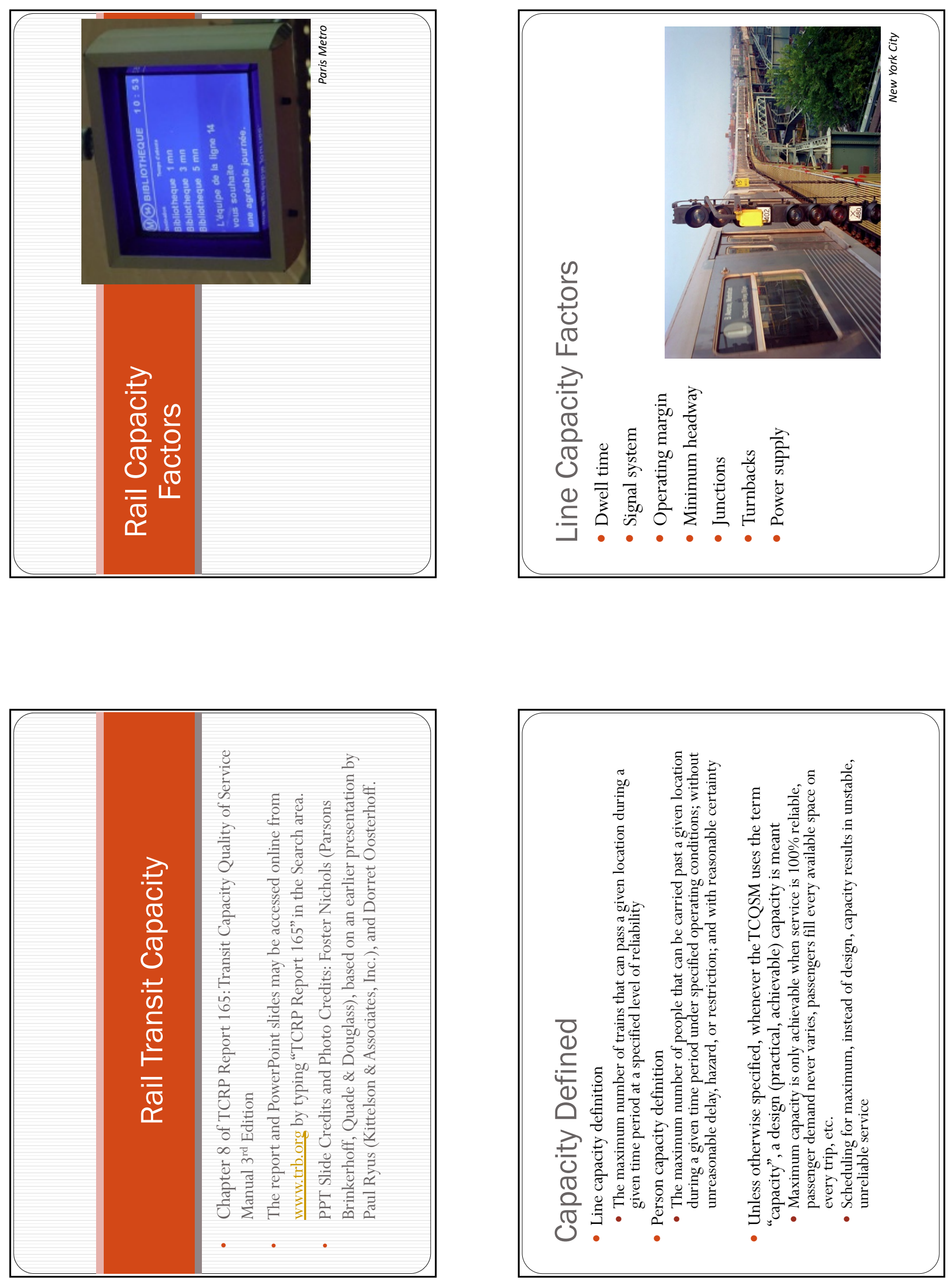

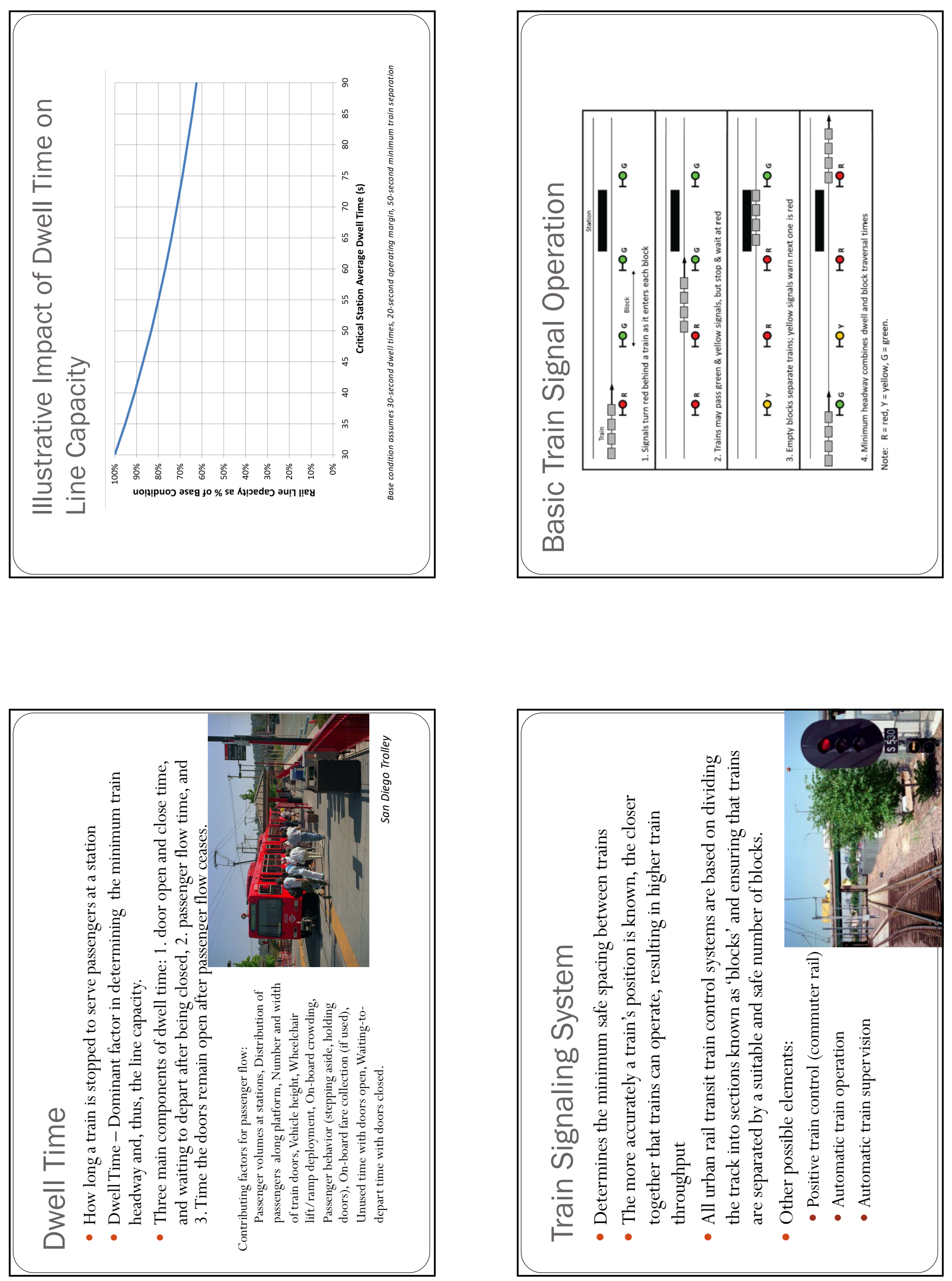

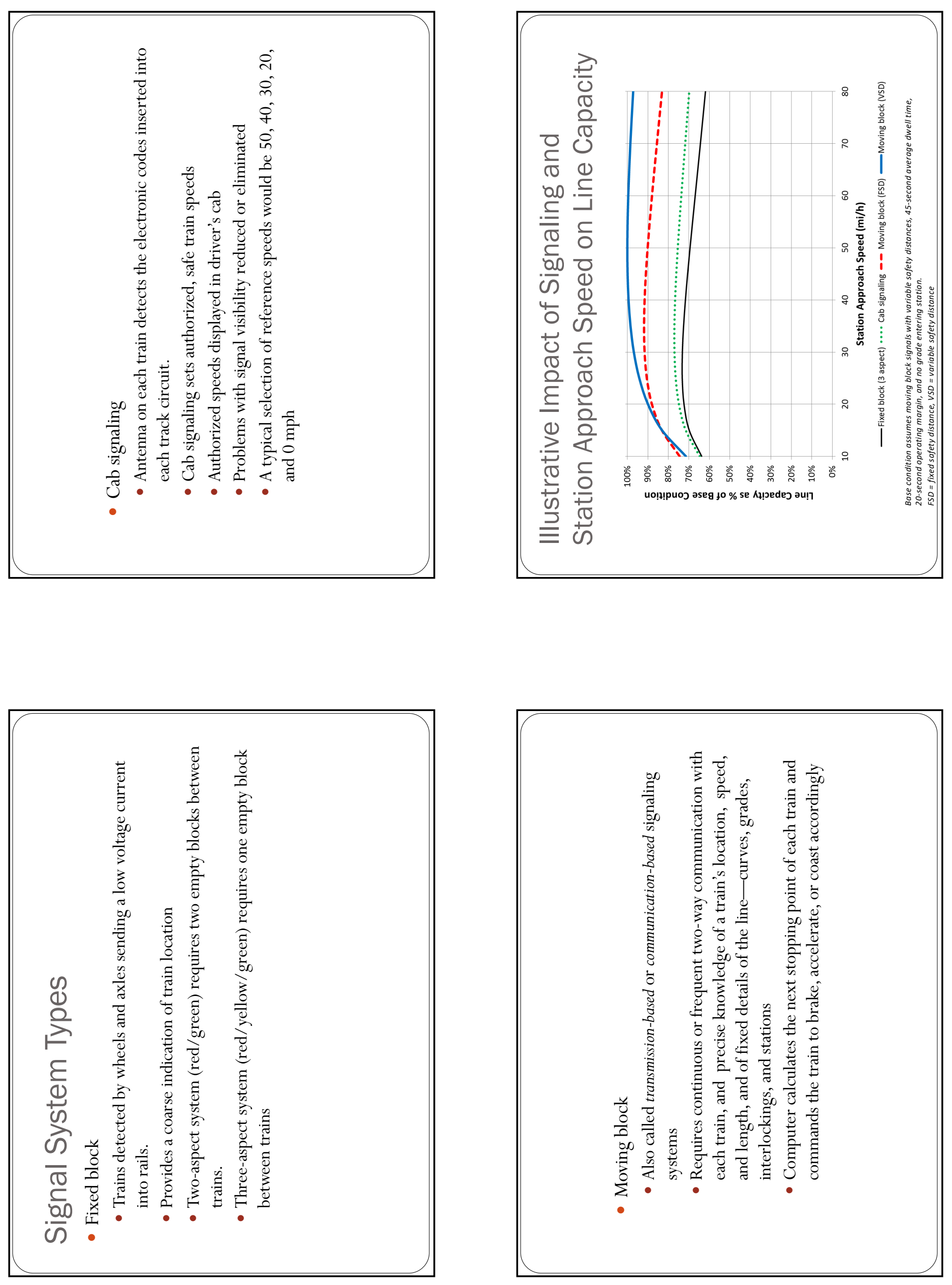

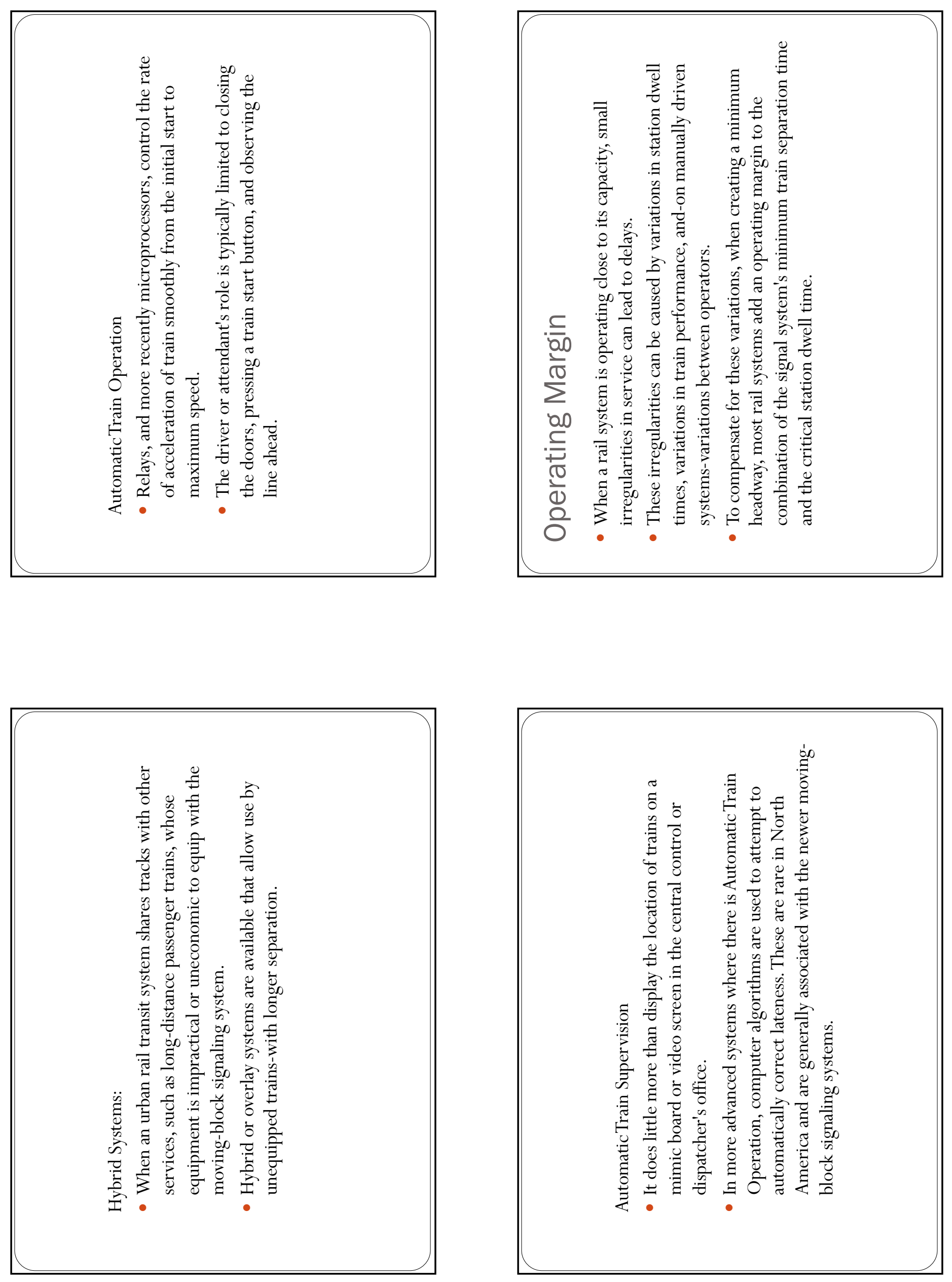

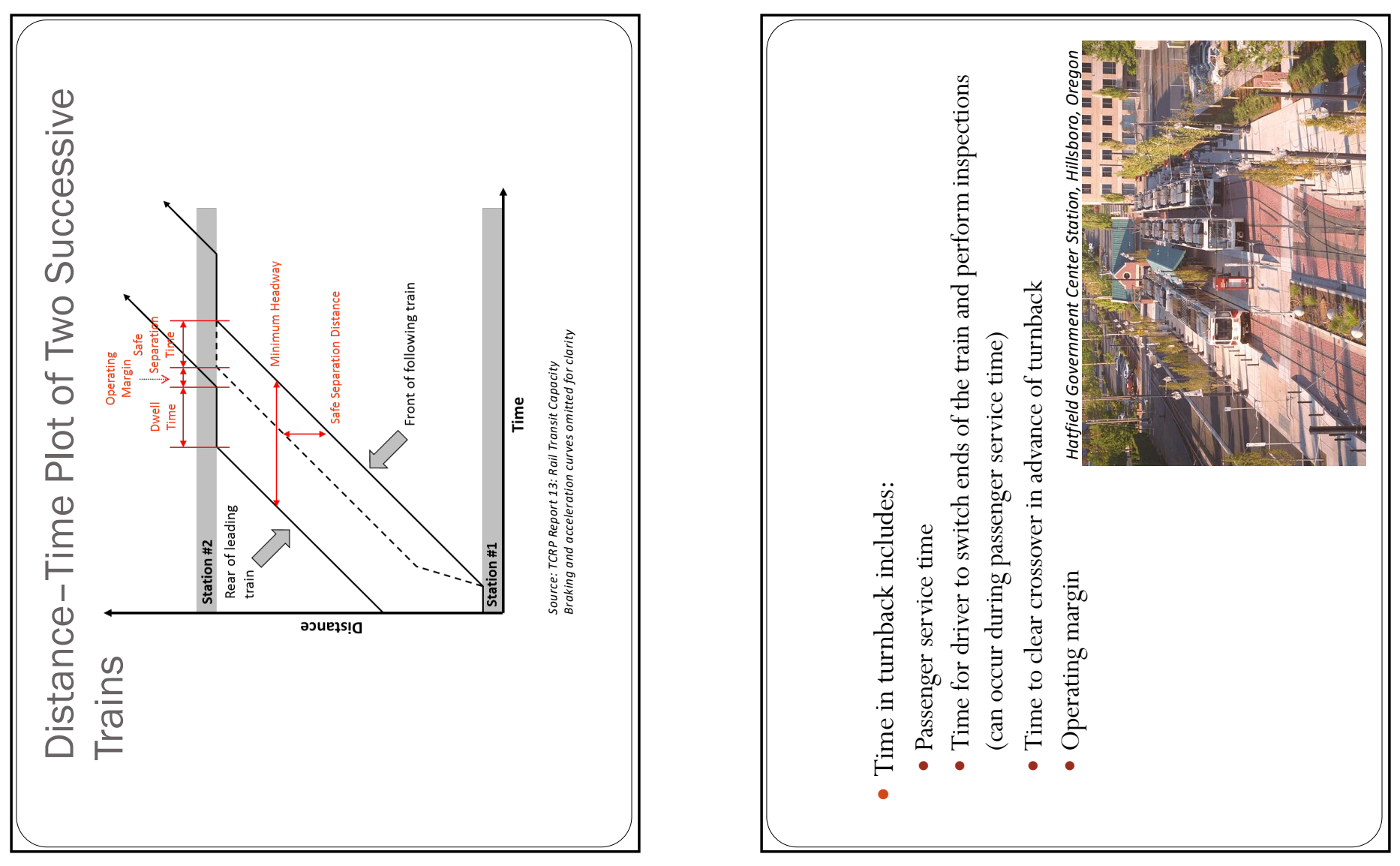

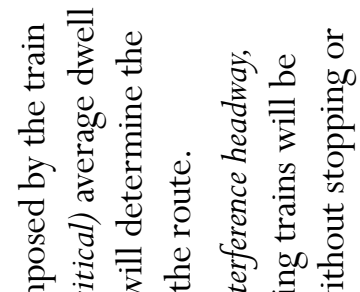

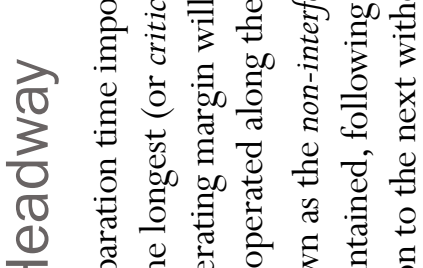

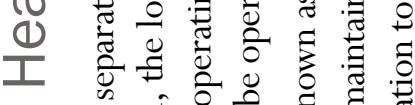

U)

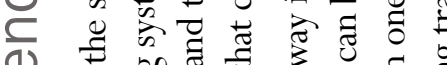

(1) 可

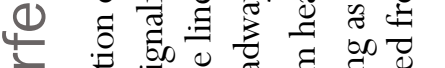

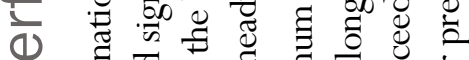

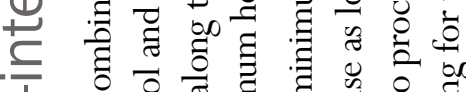

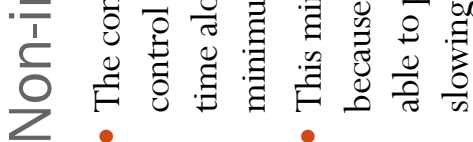

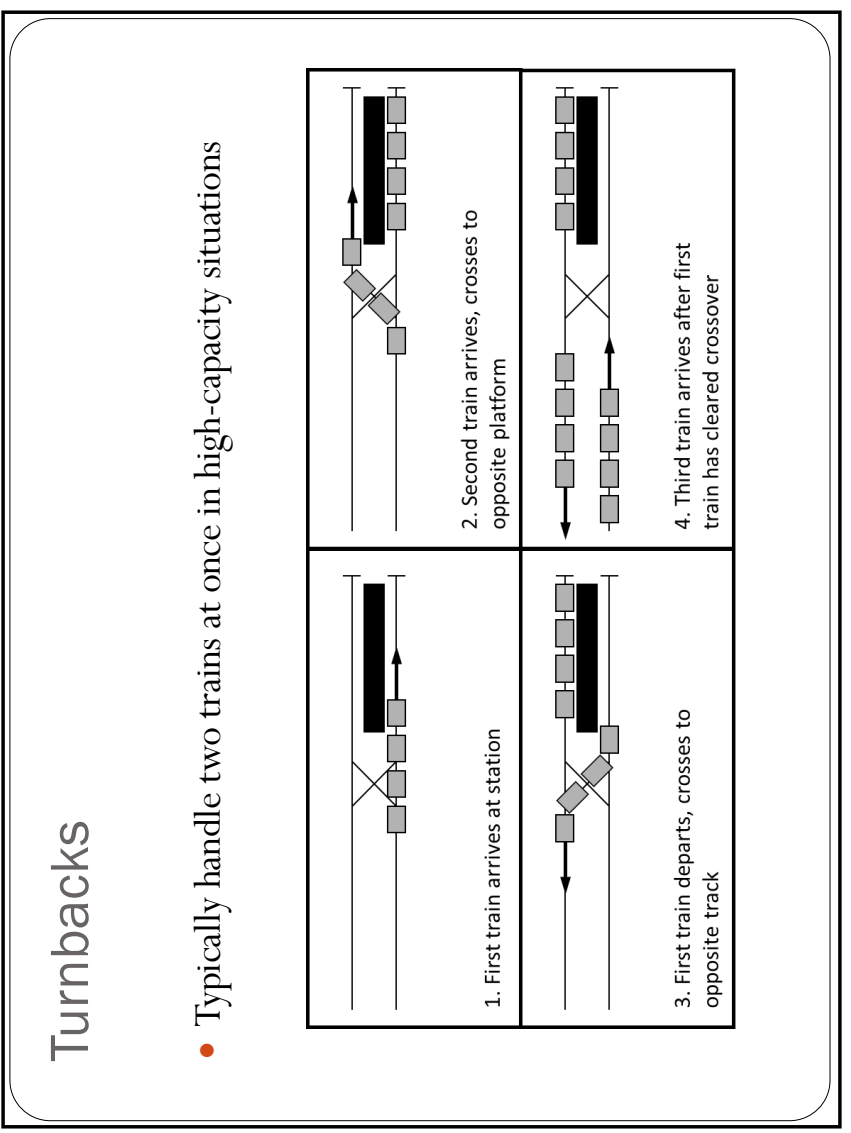



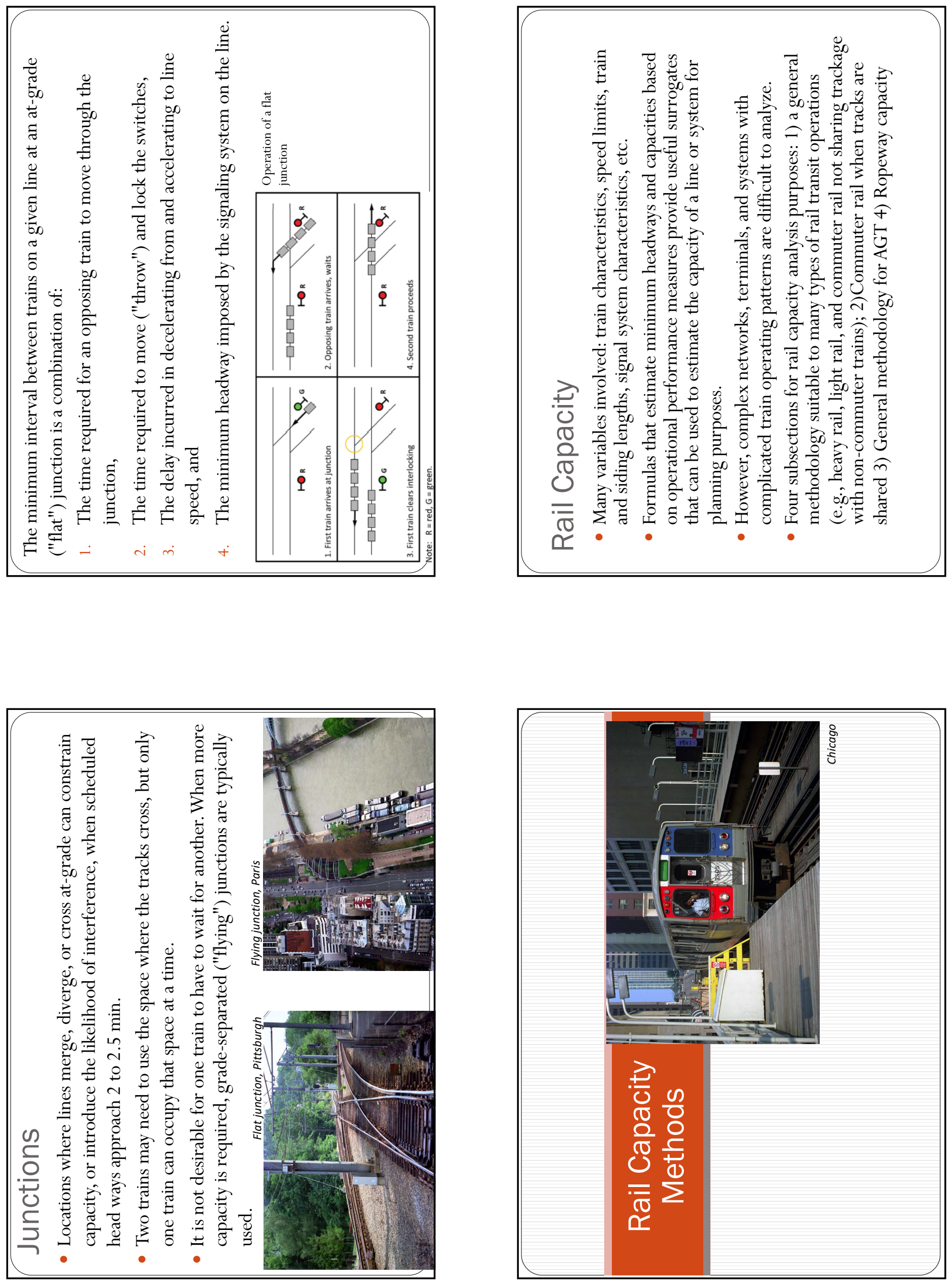

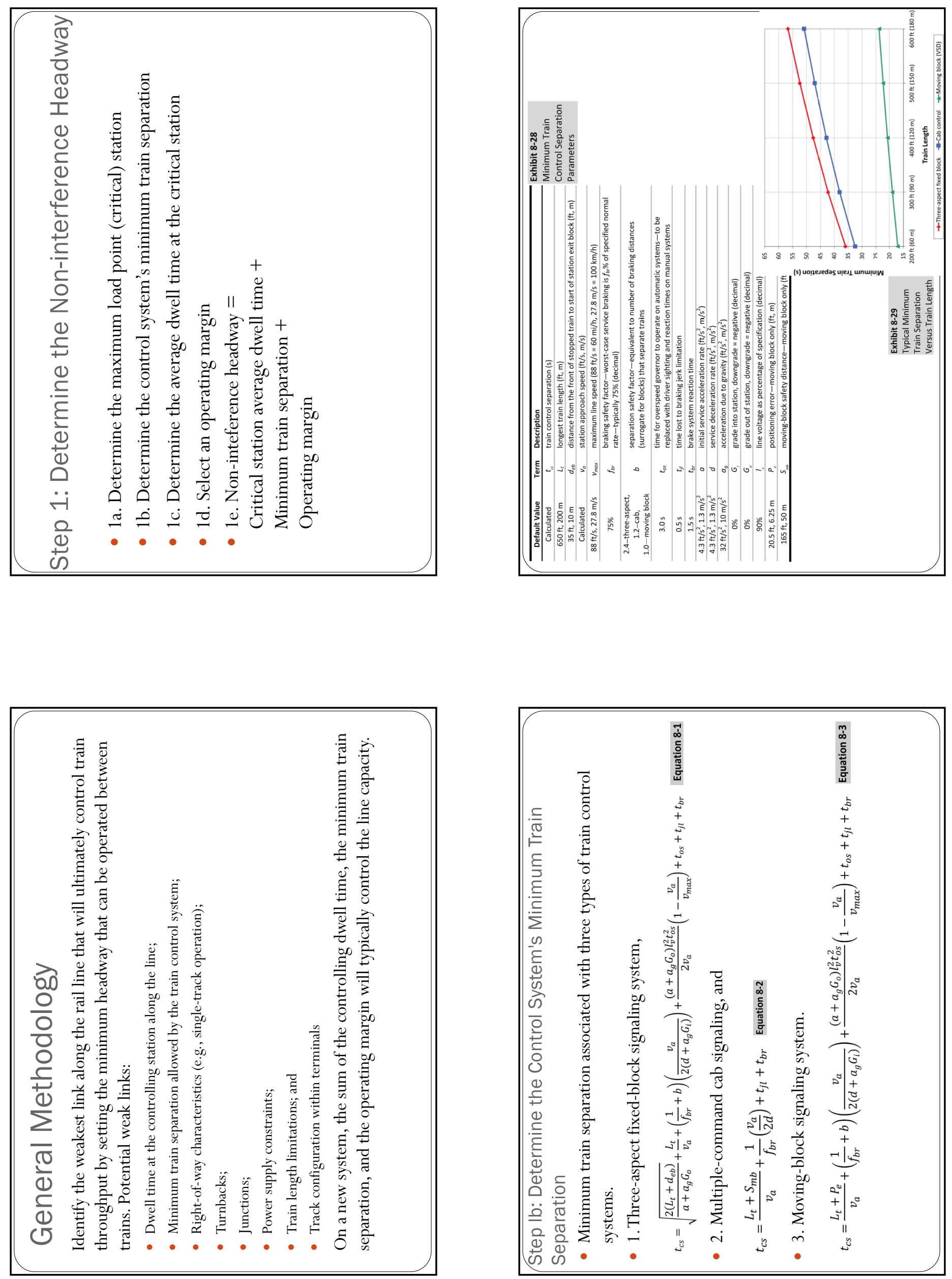

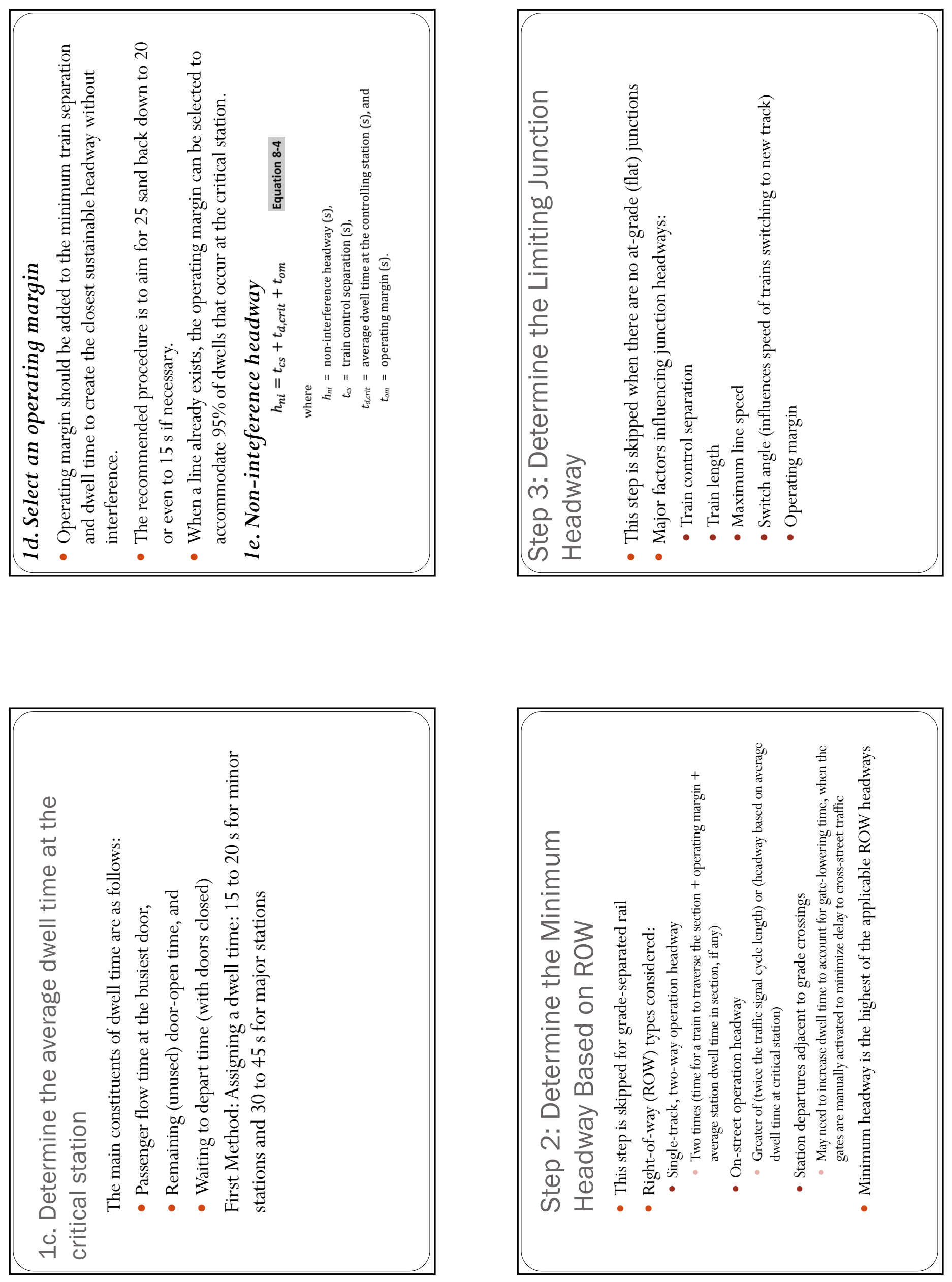

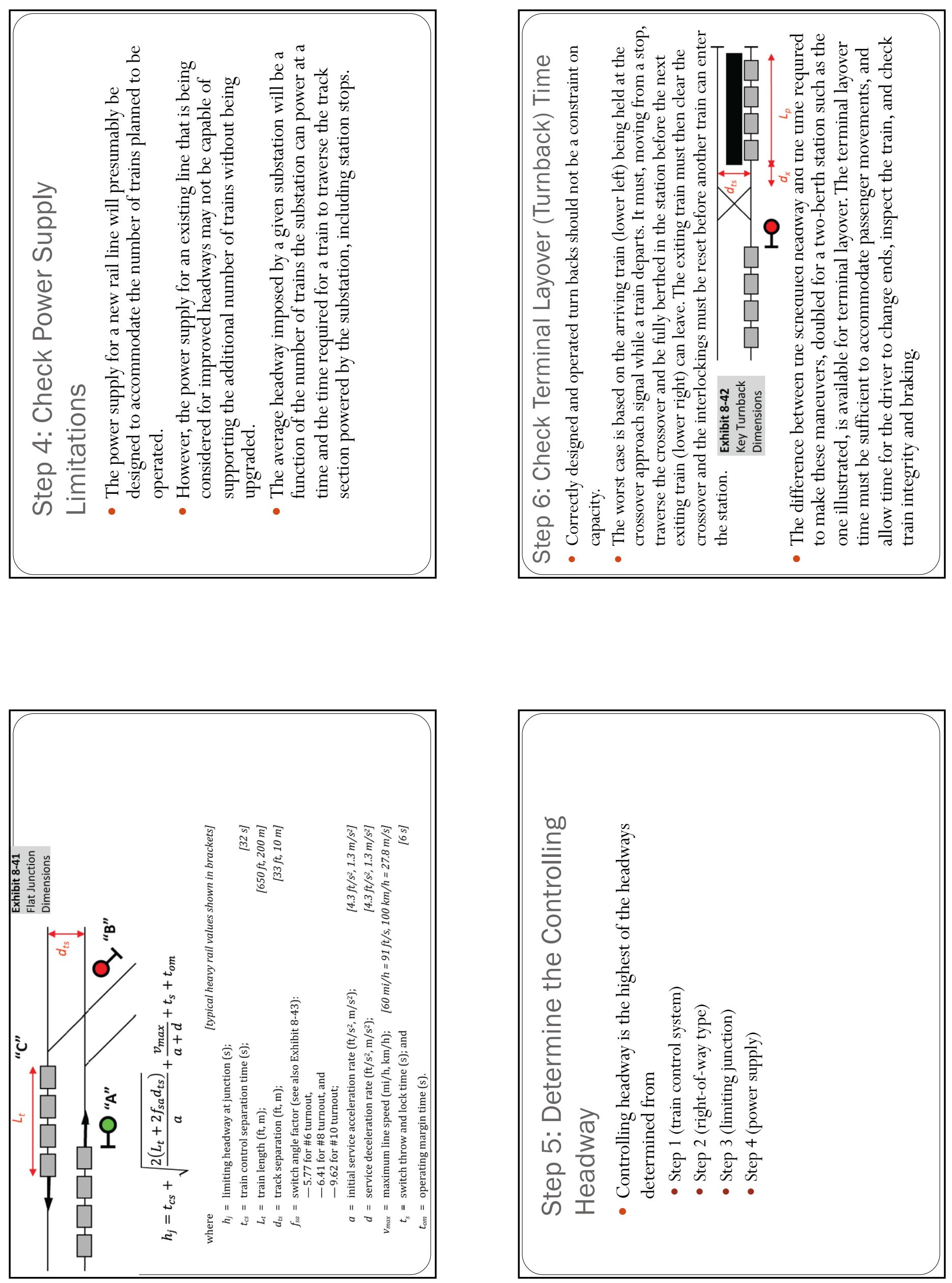

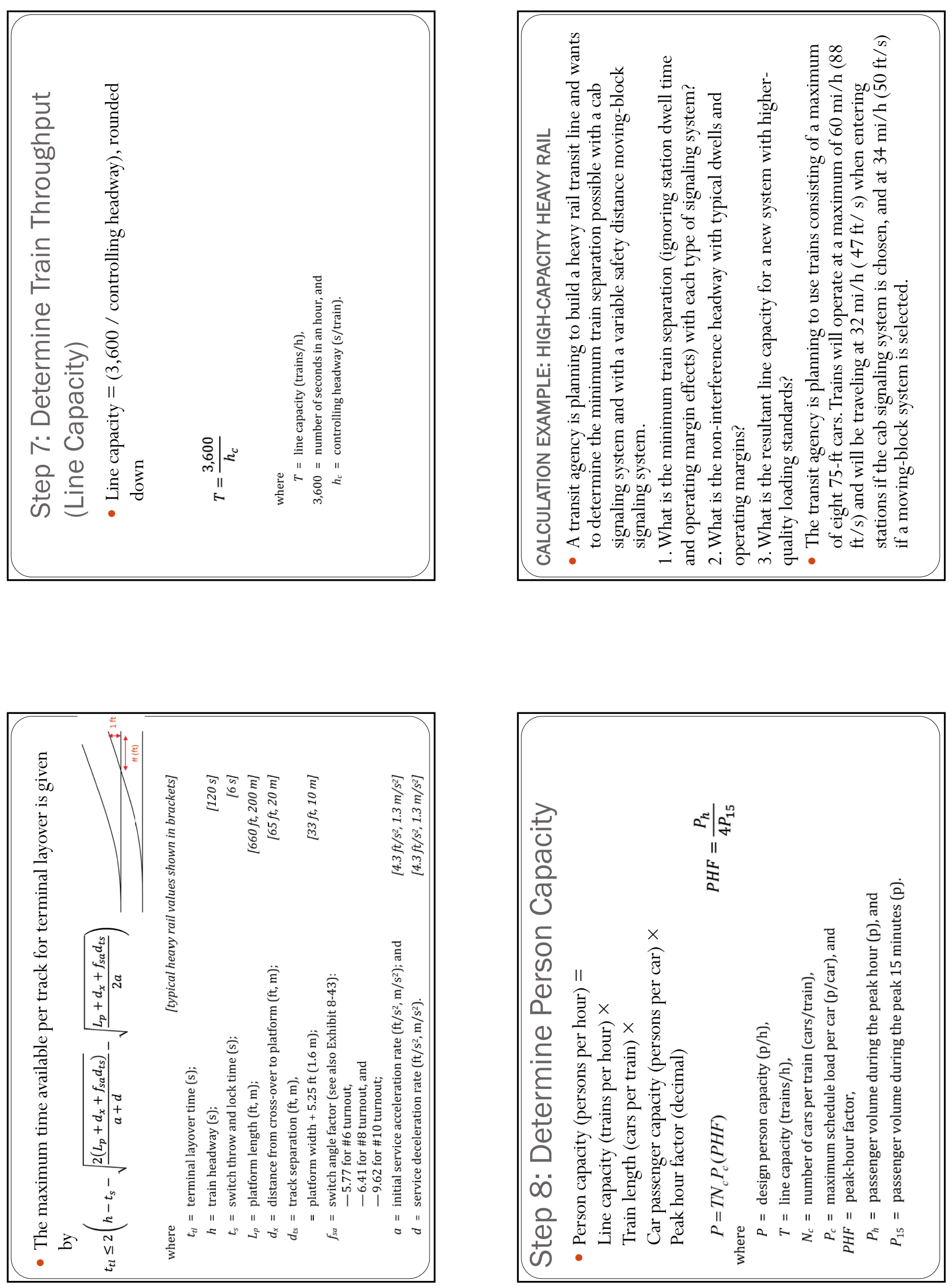

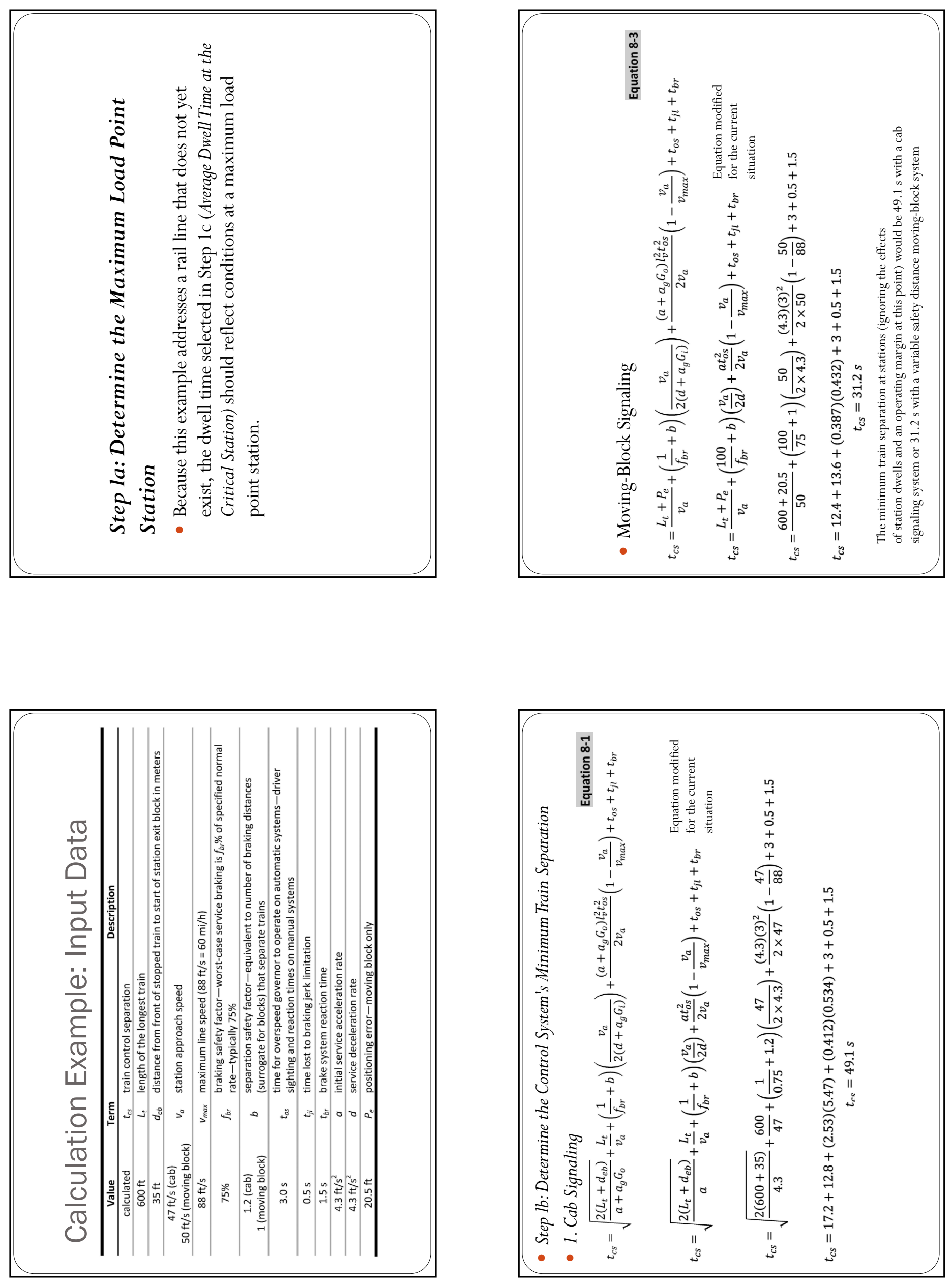

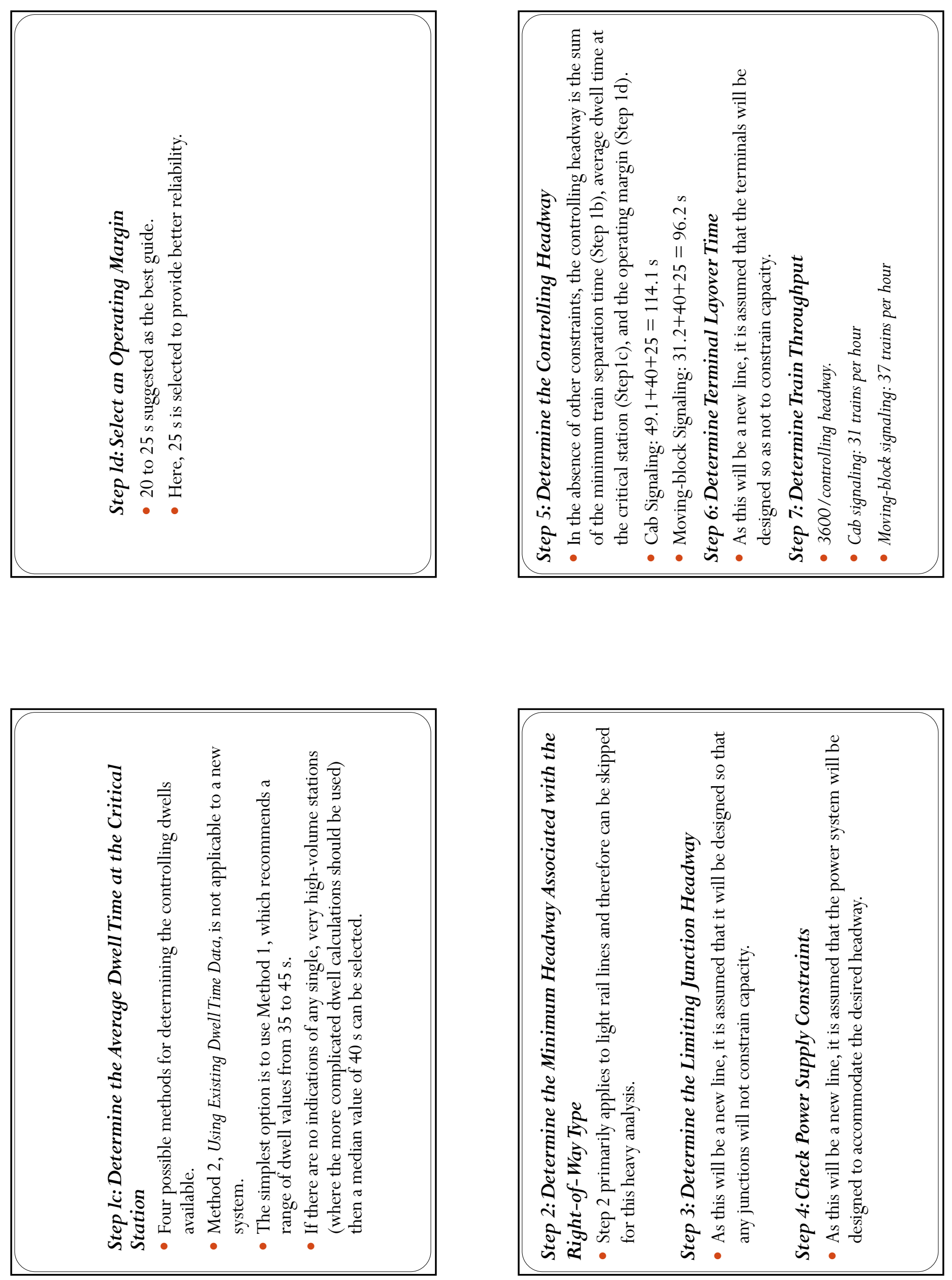

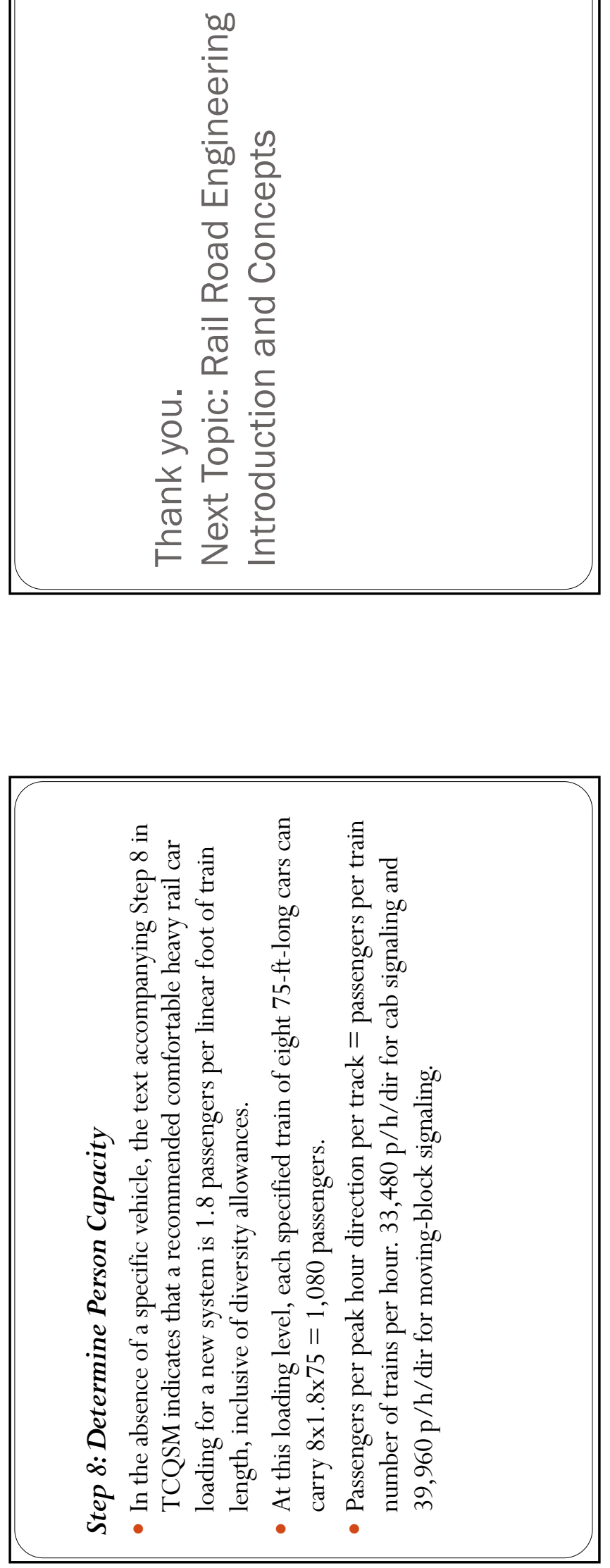

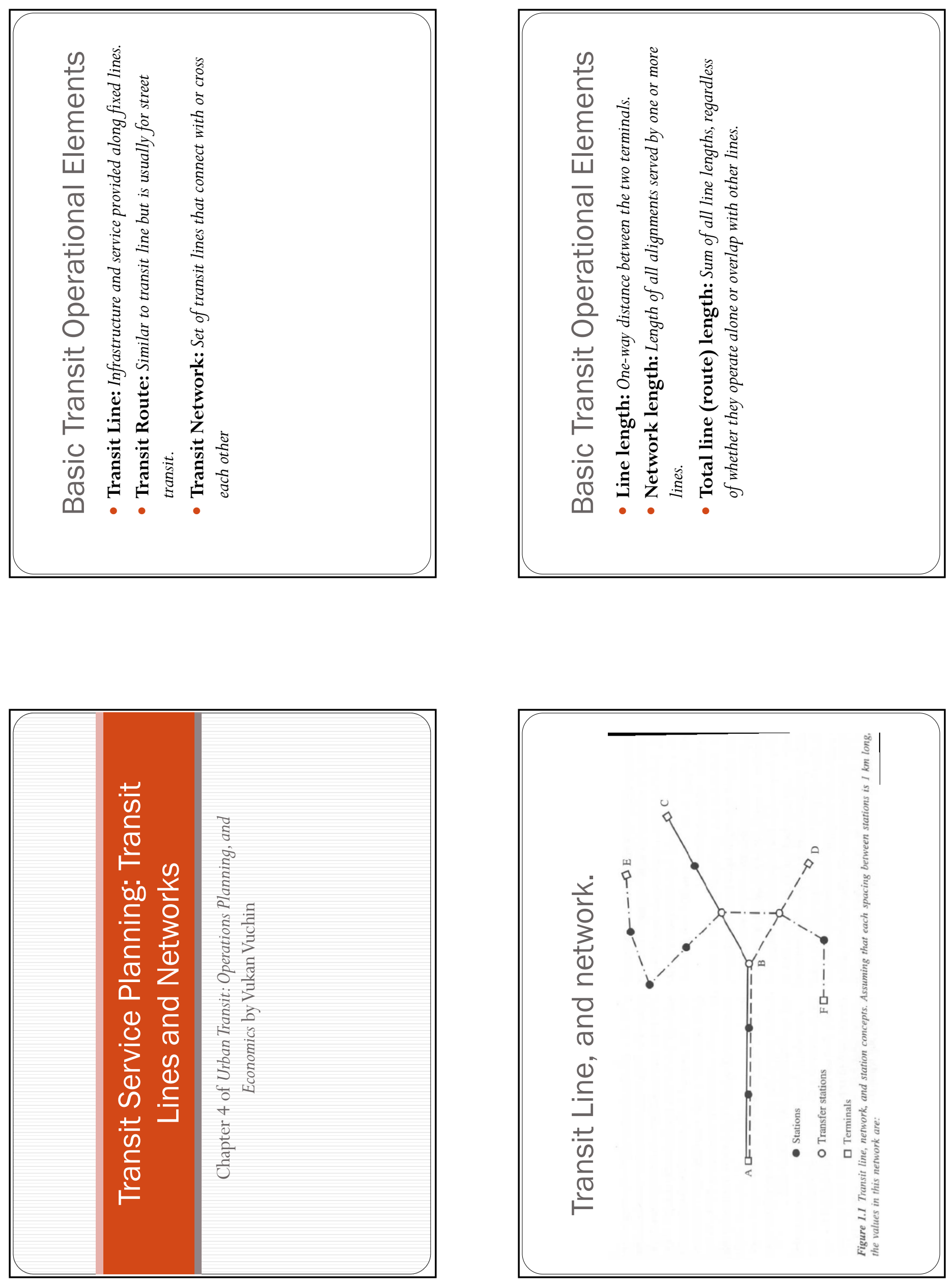

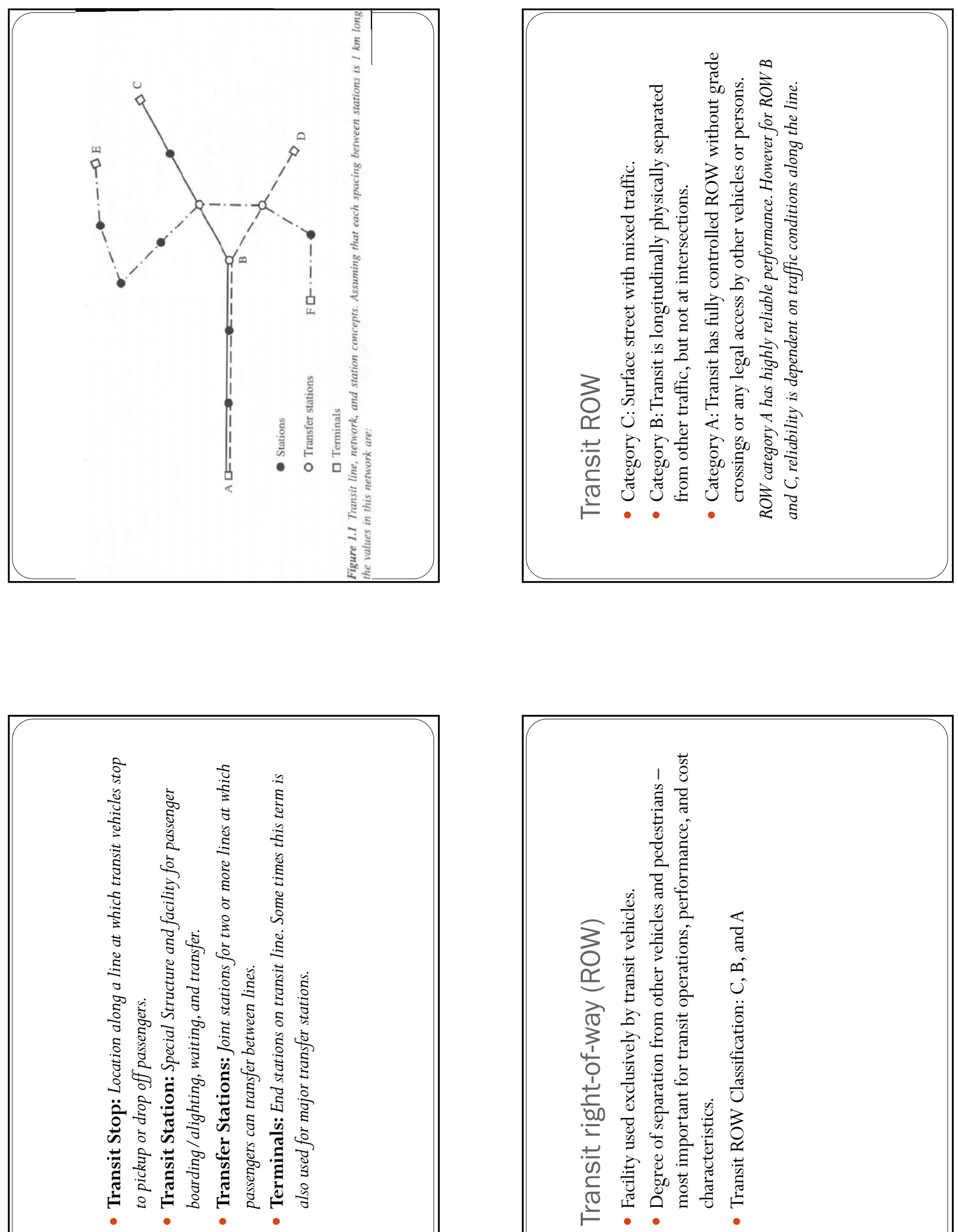

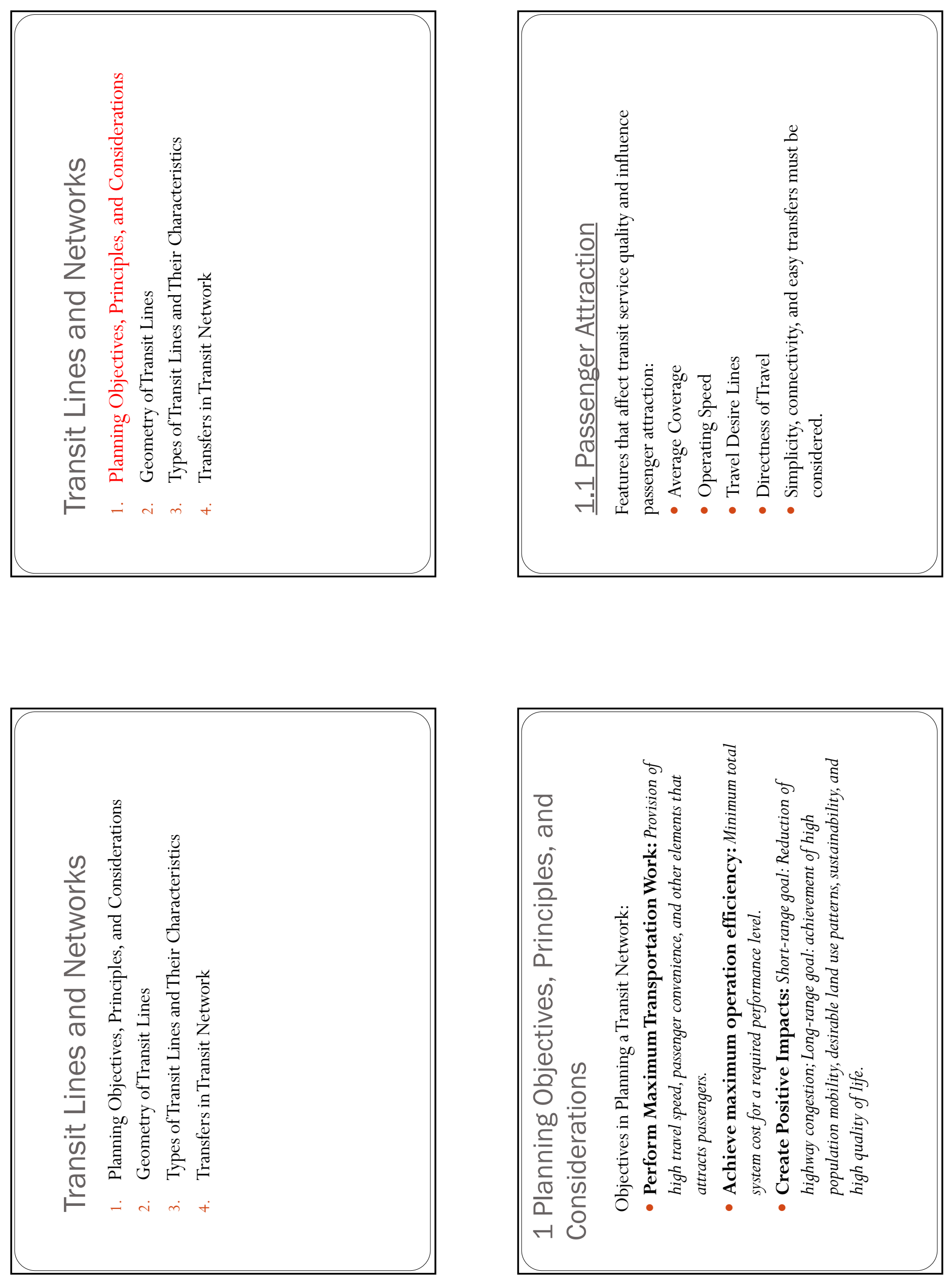

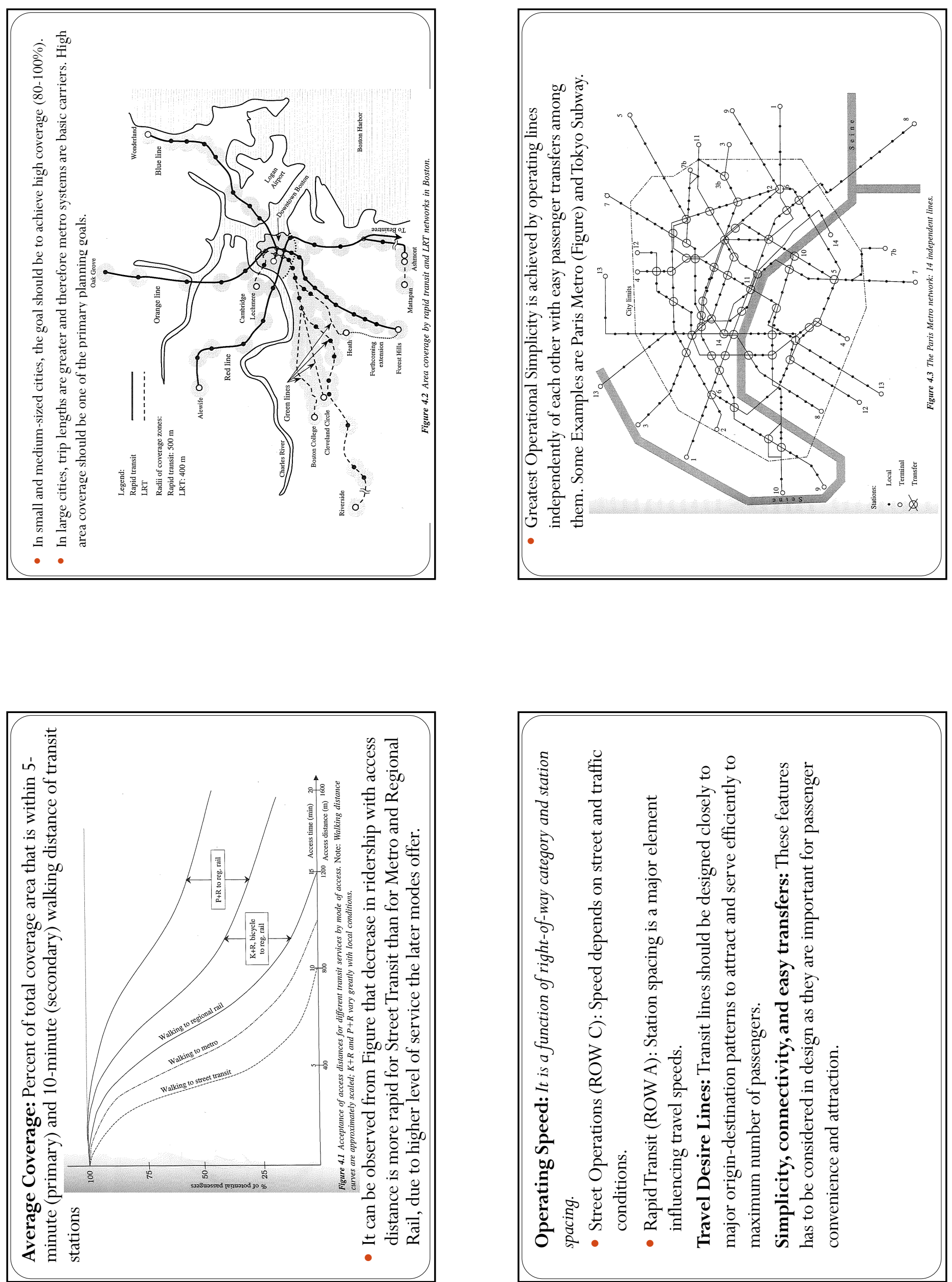

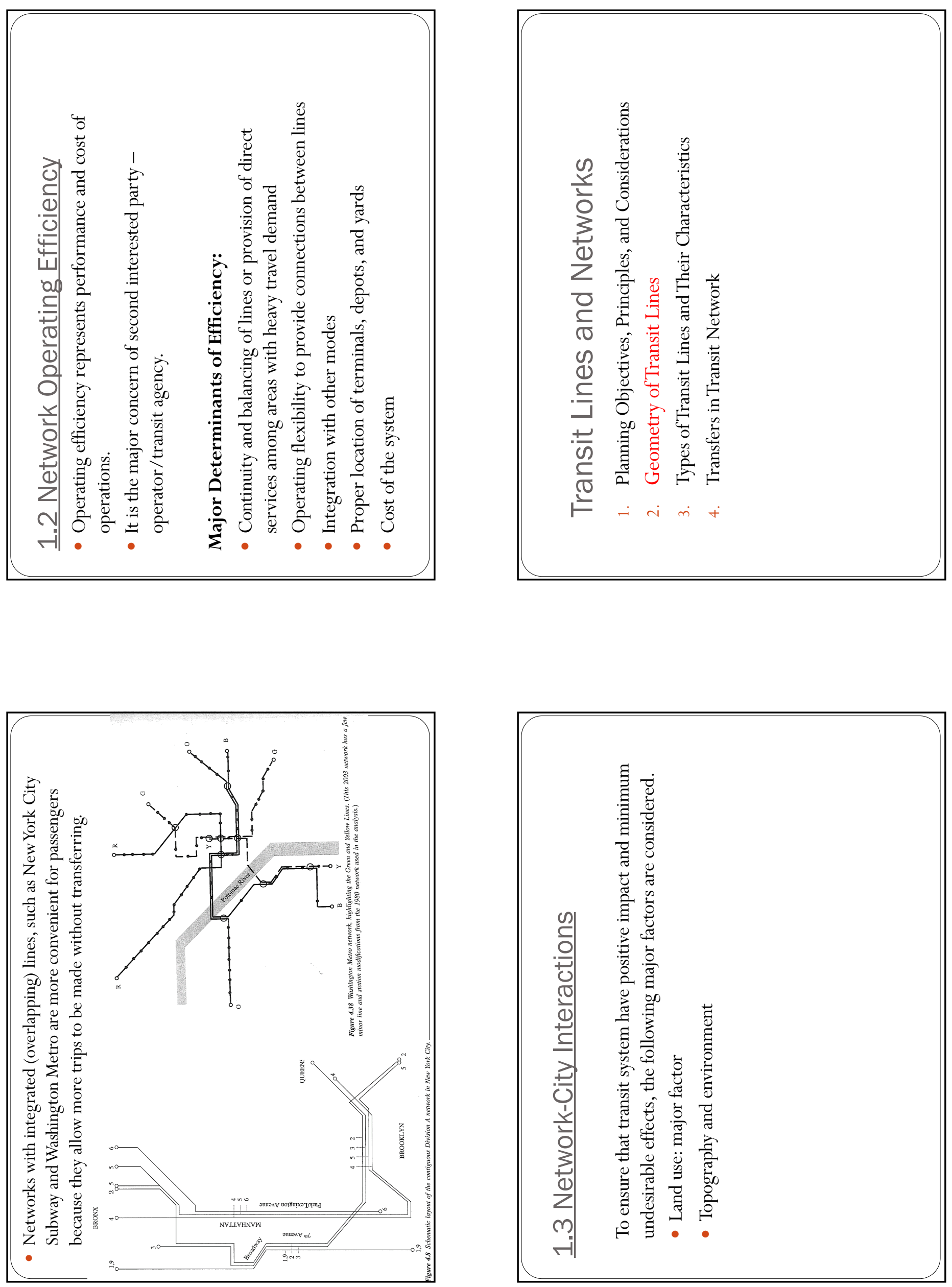

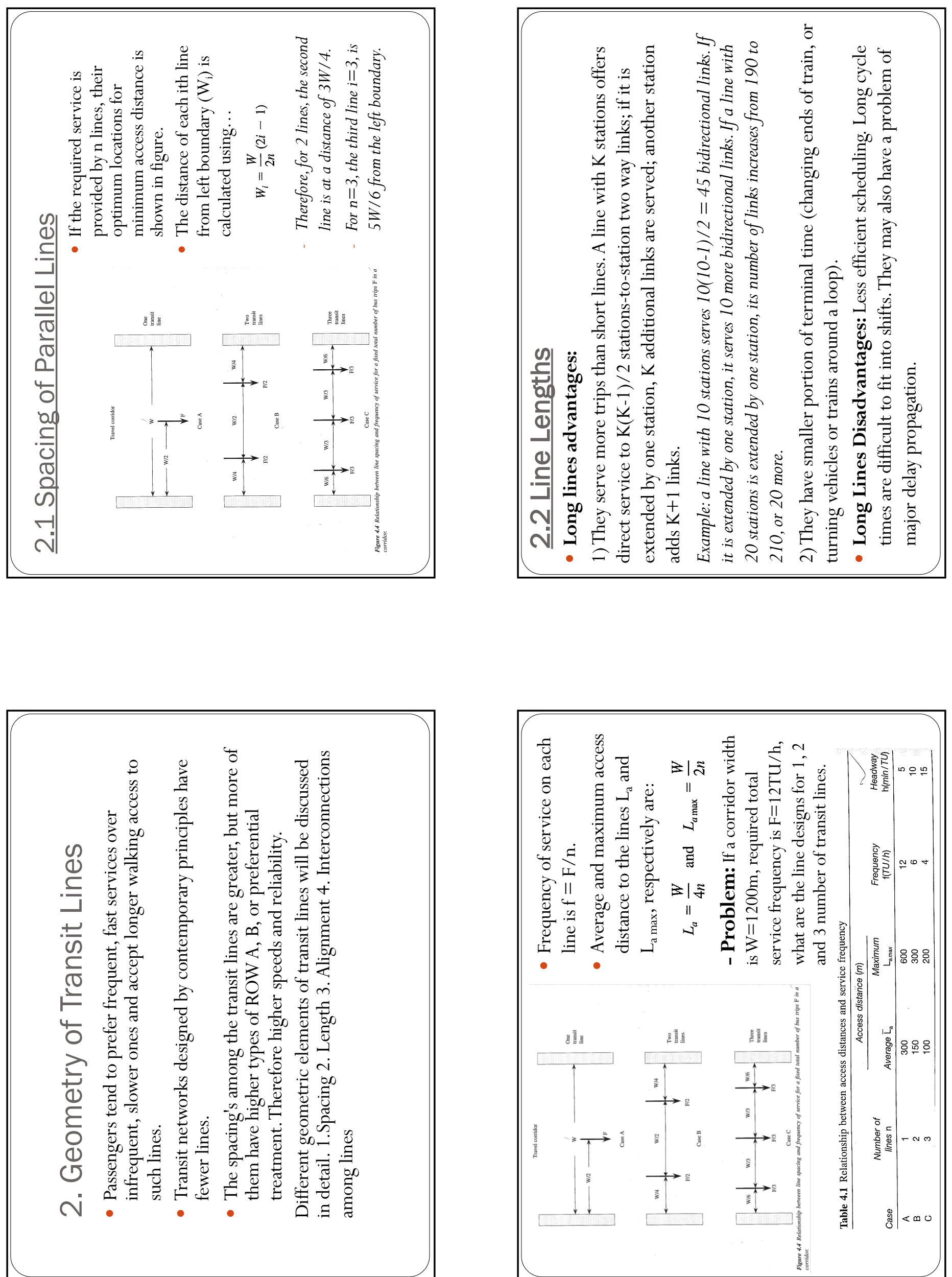

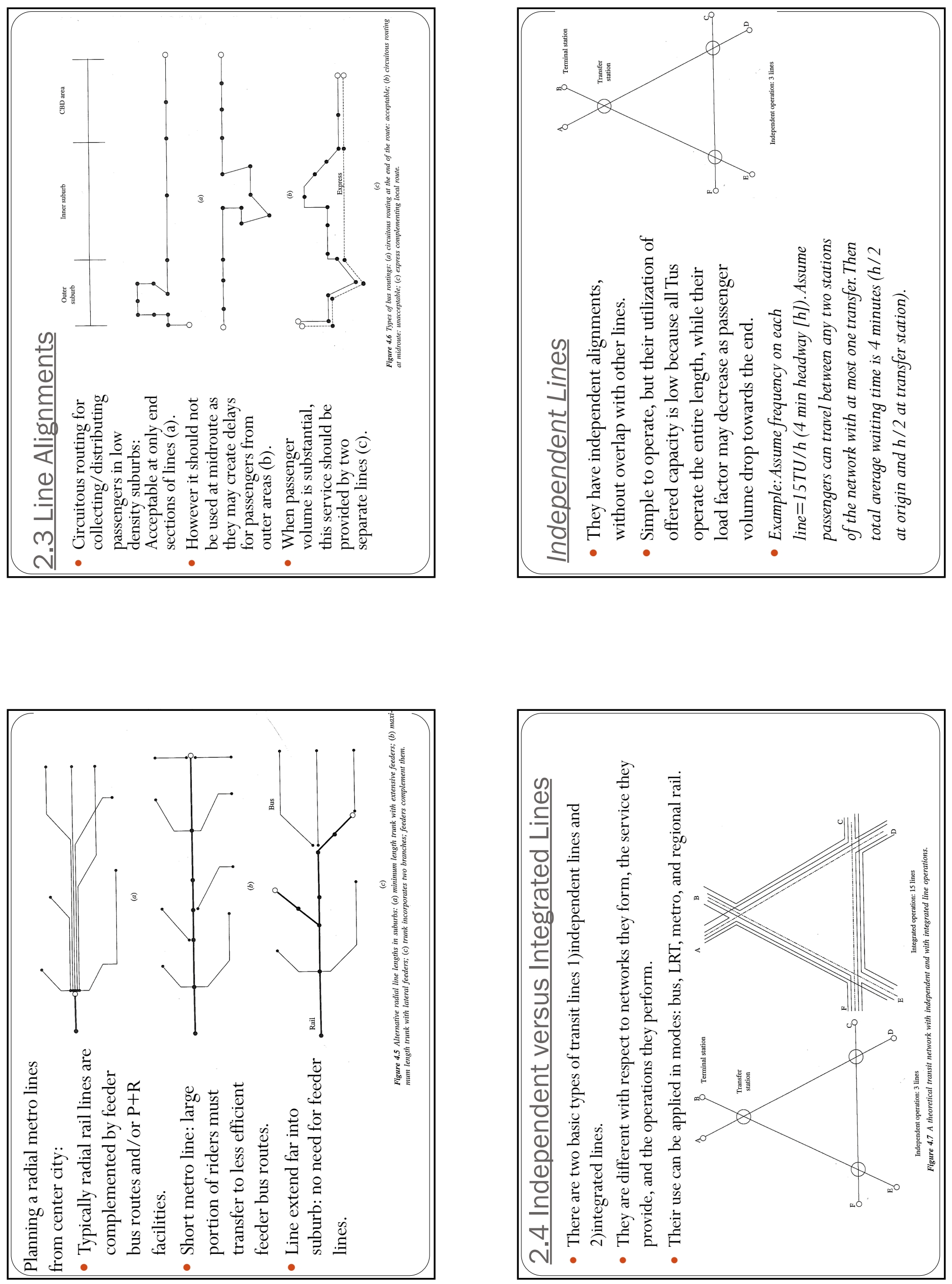

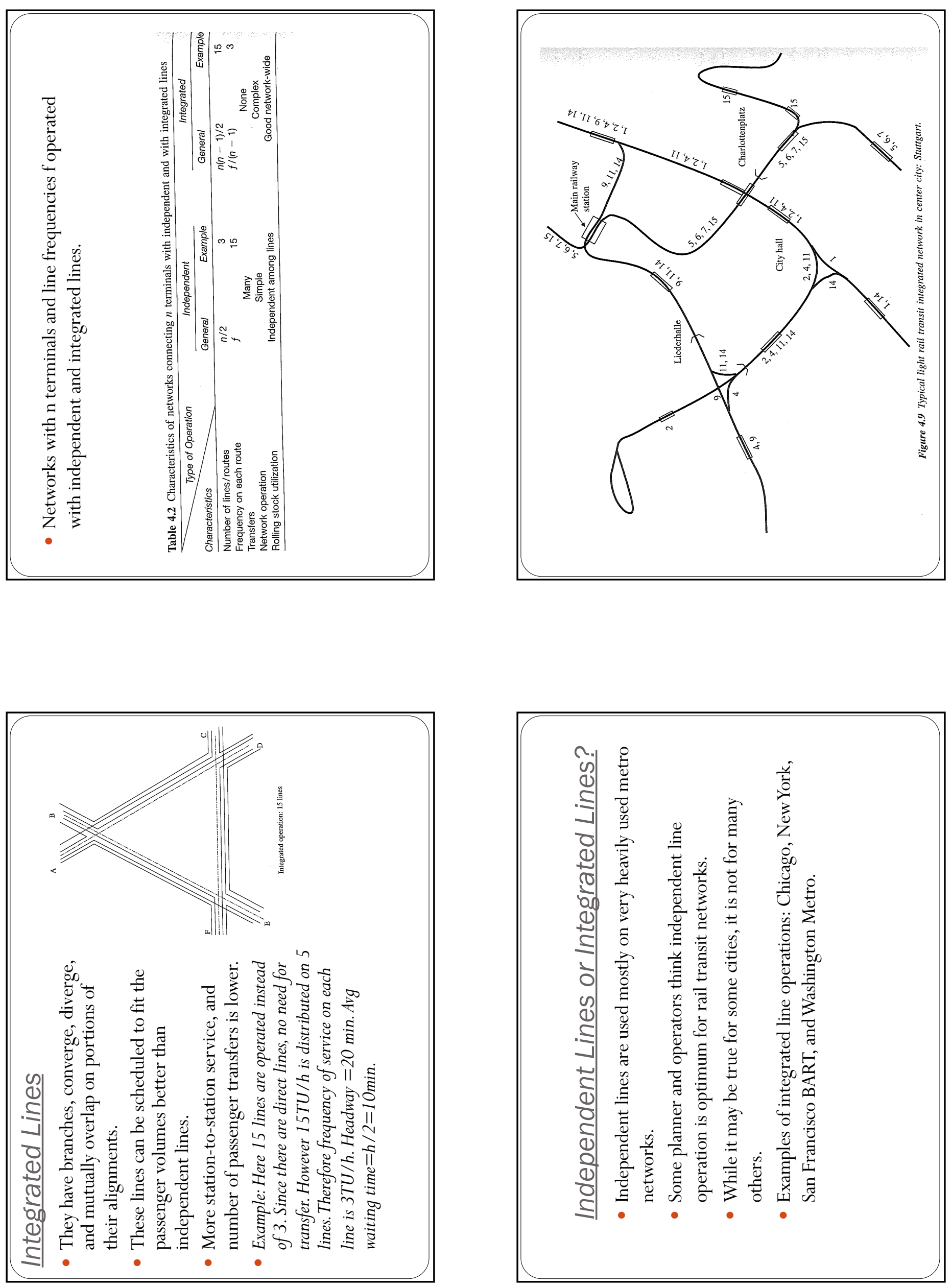

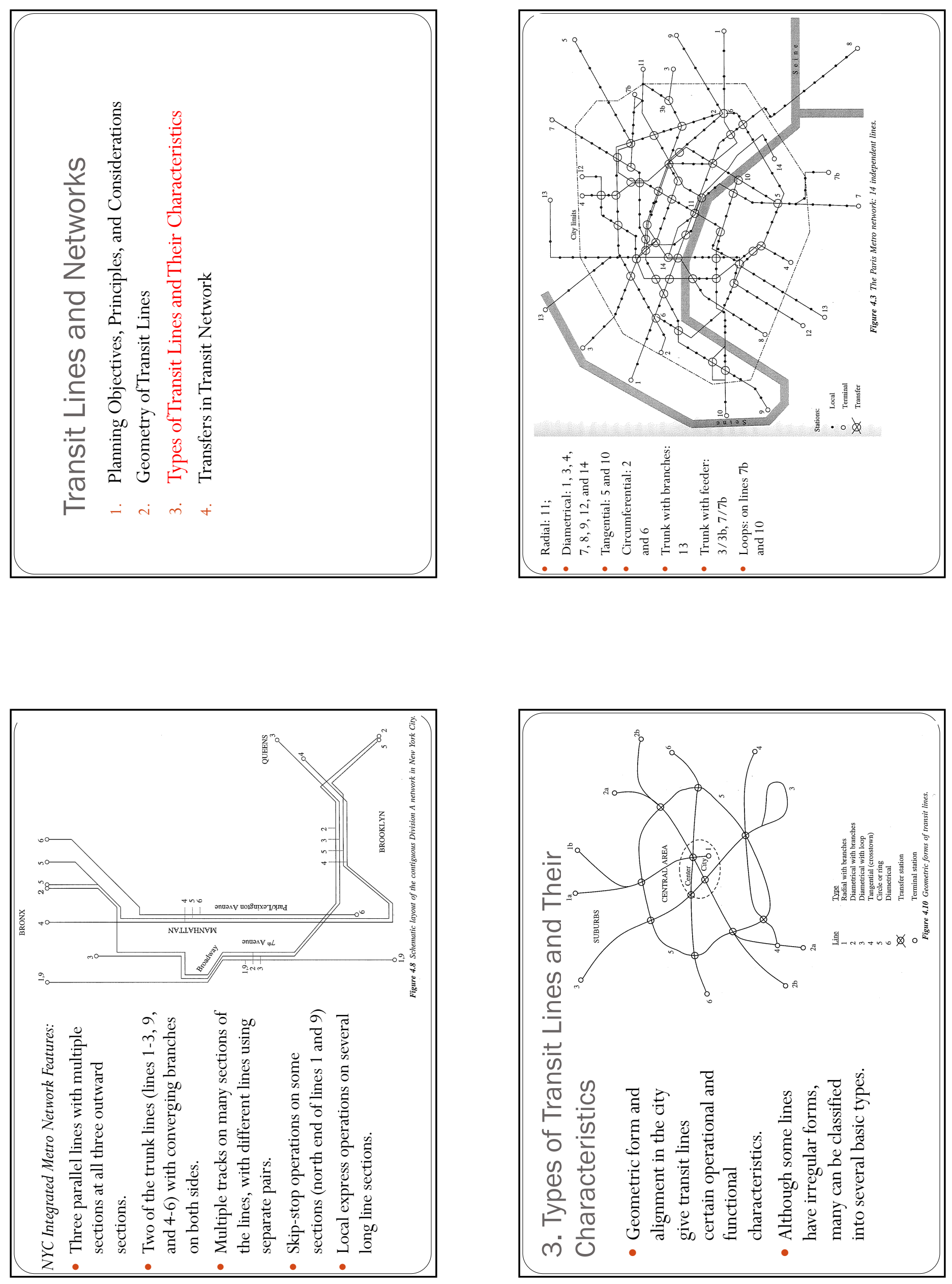

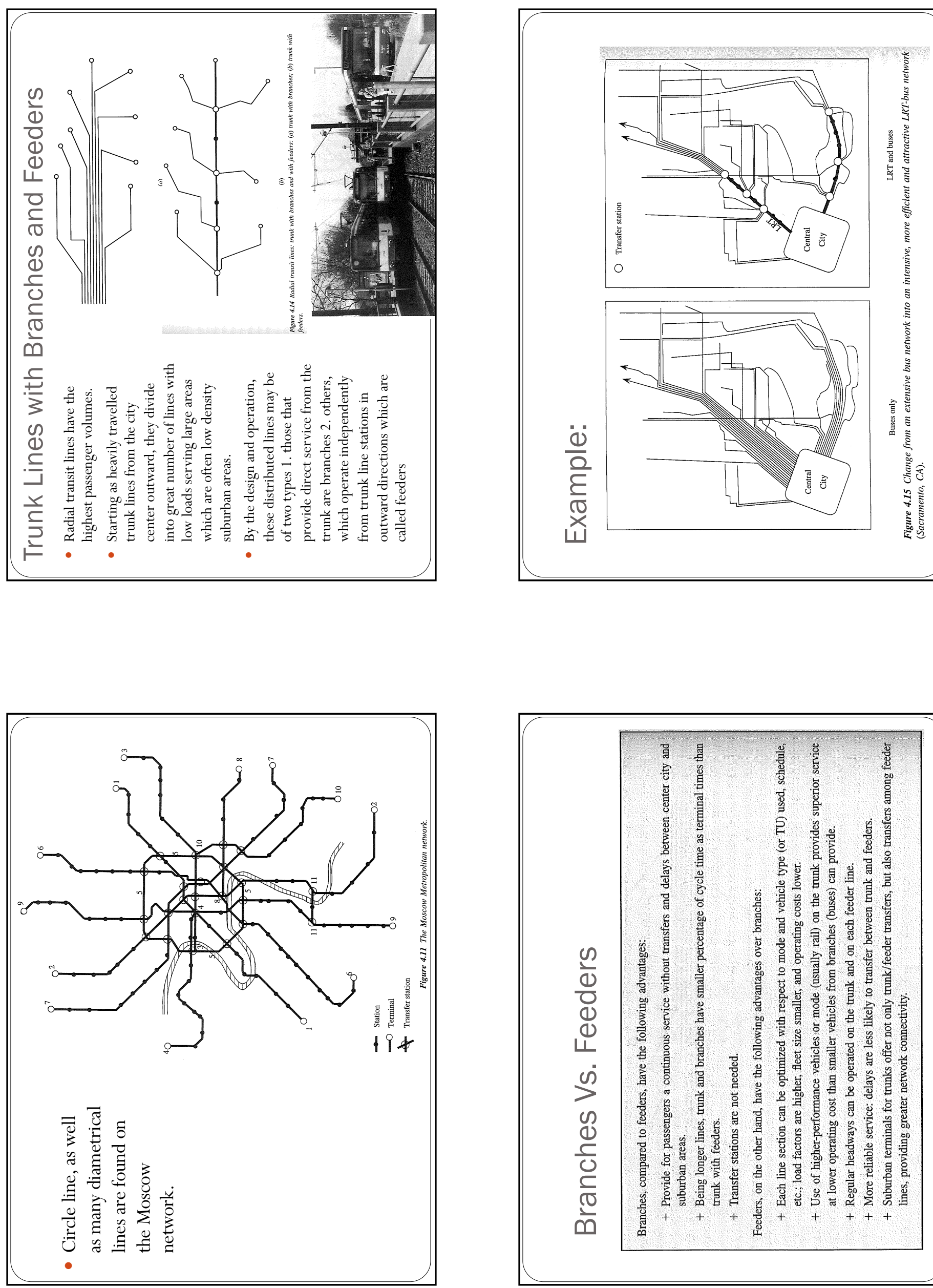


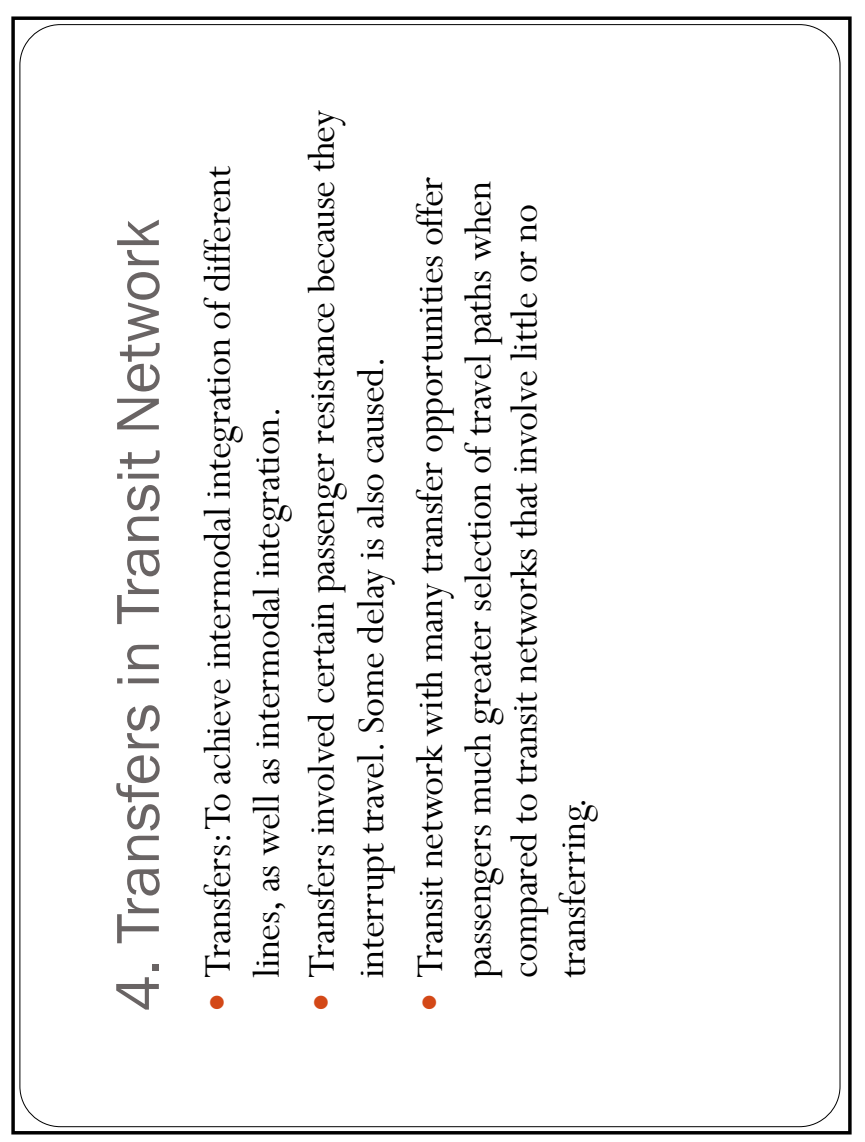



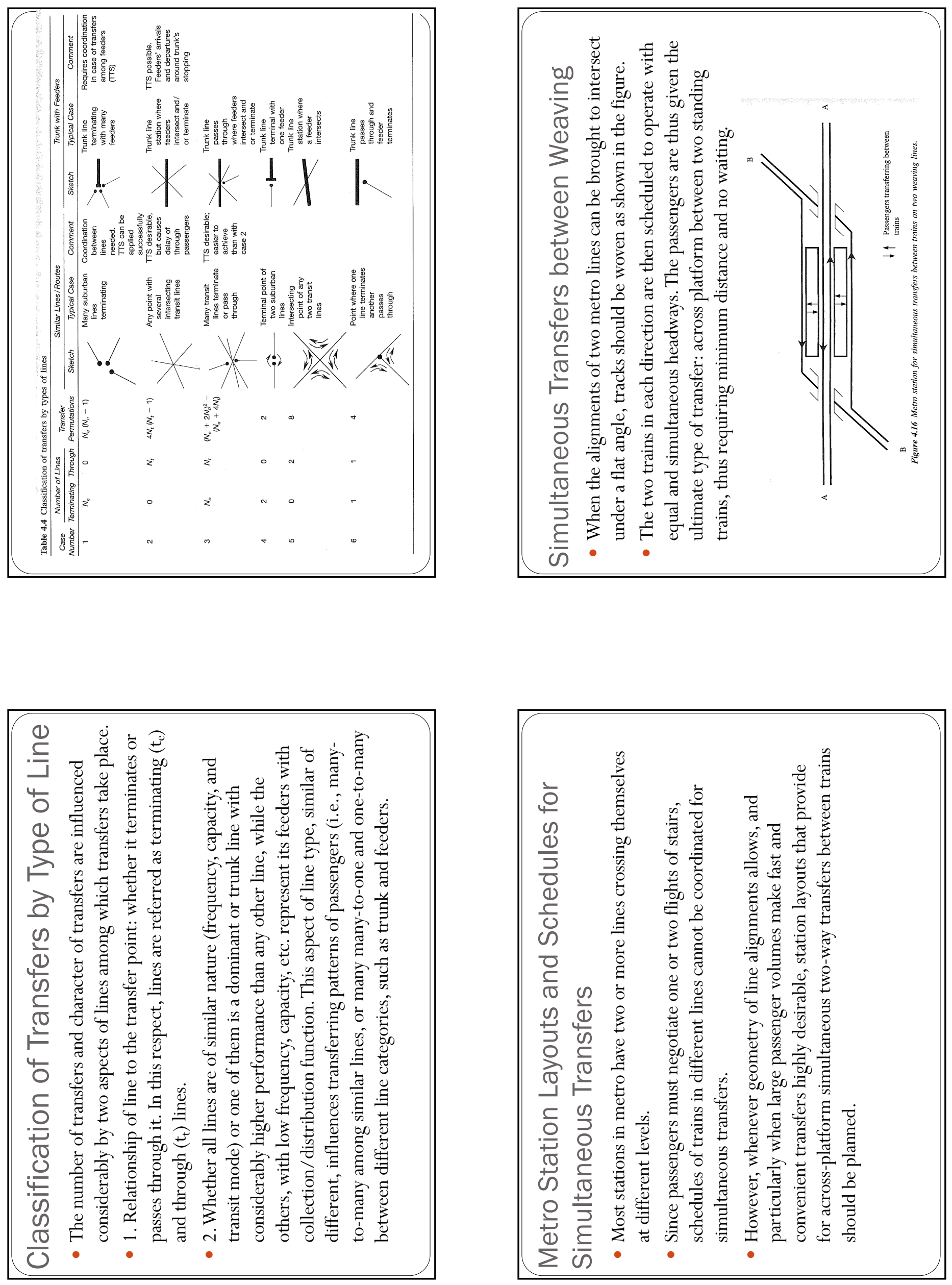

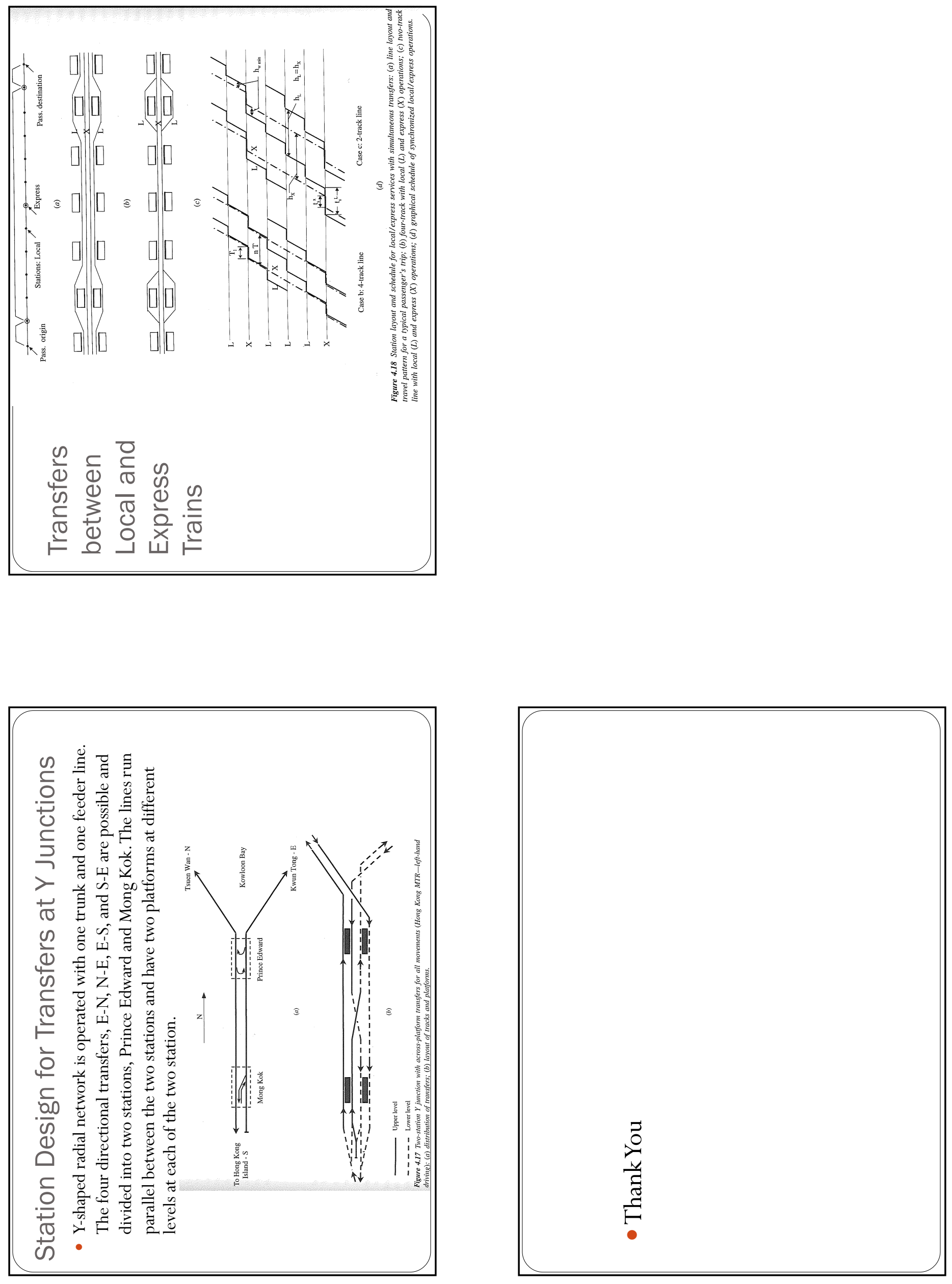

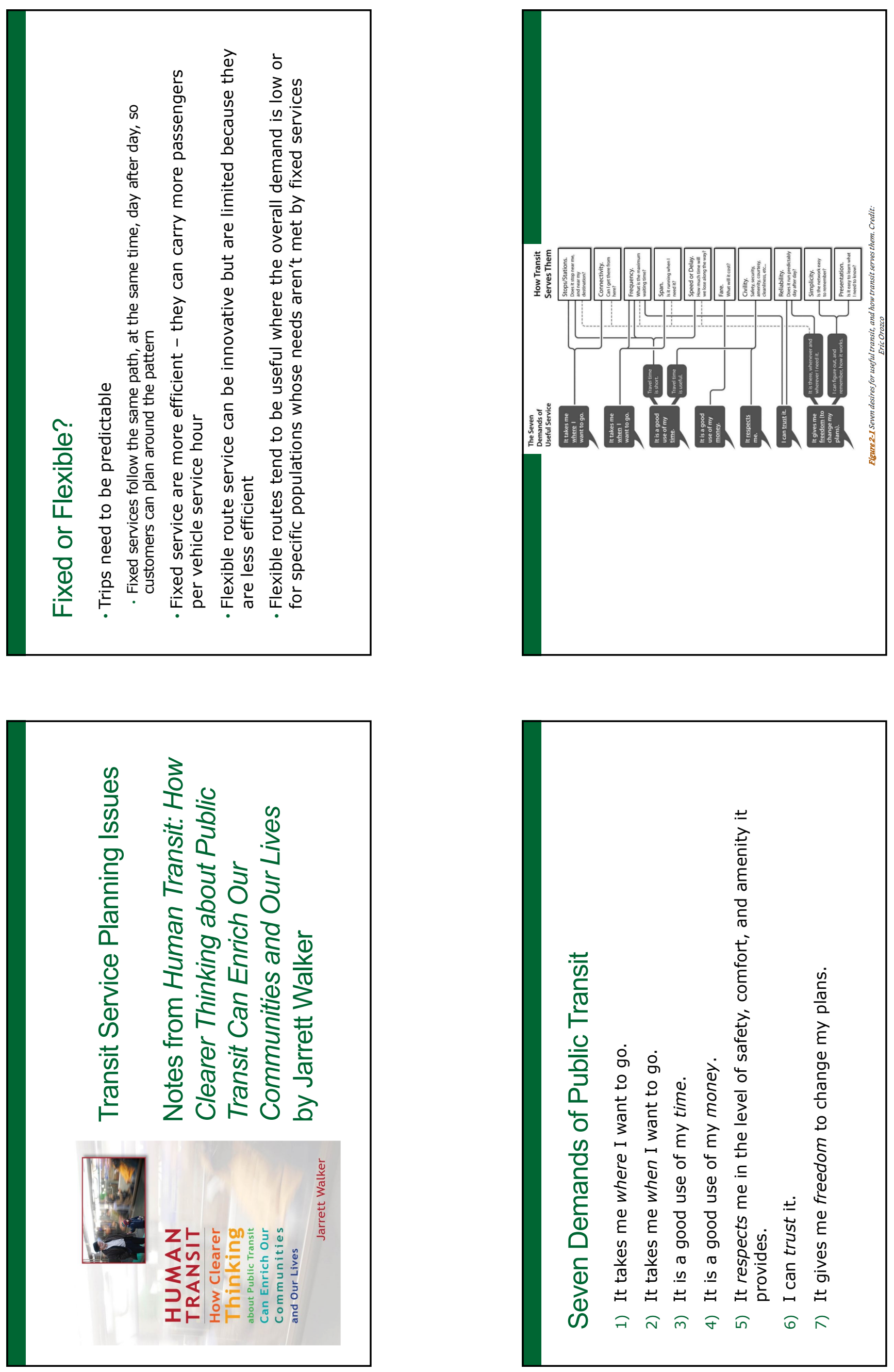

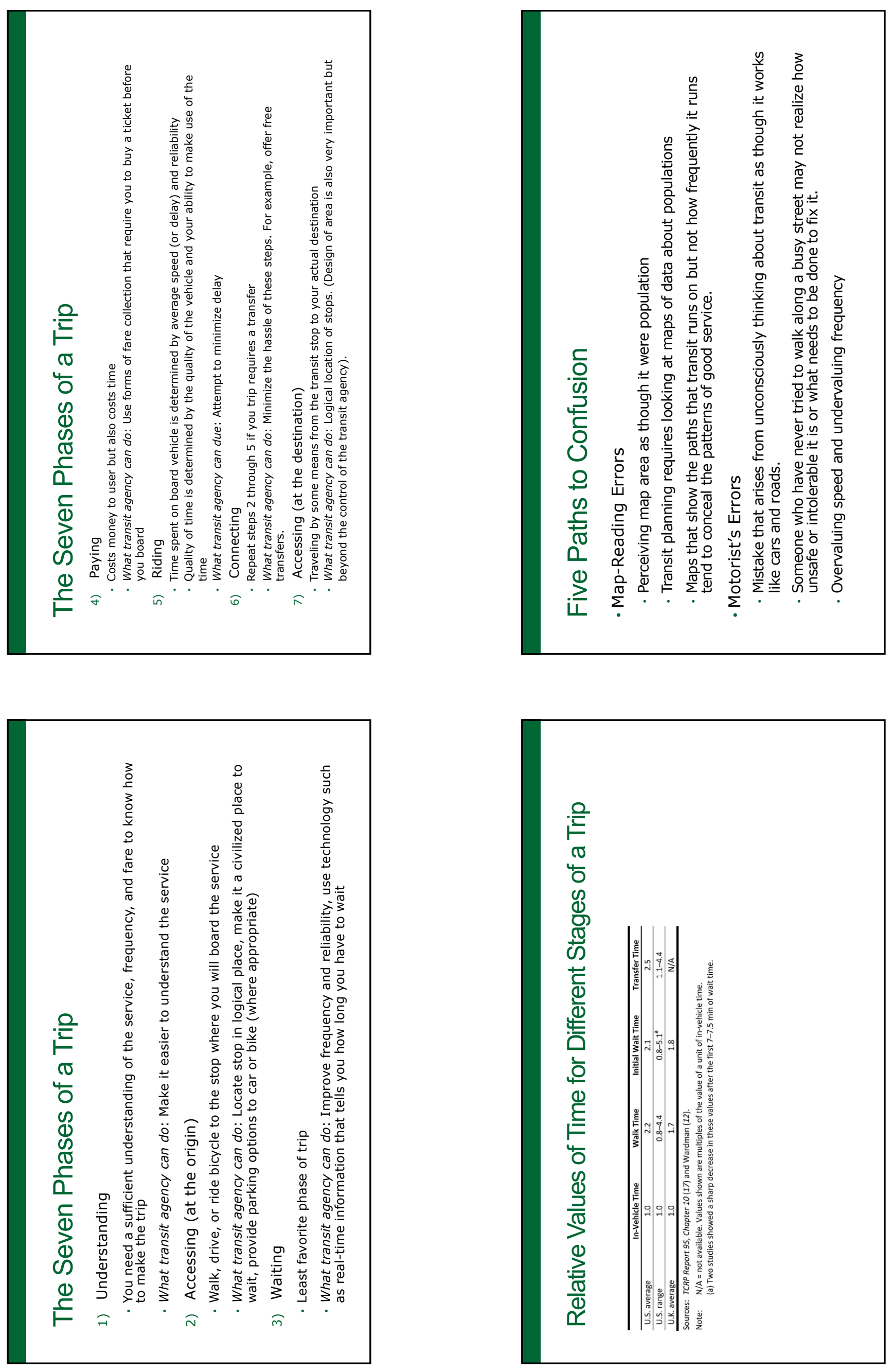

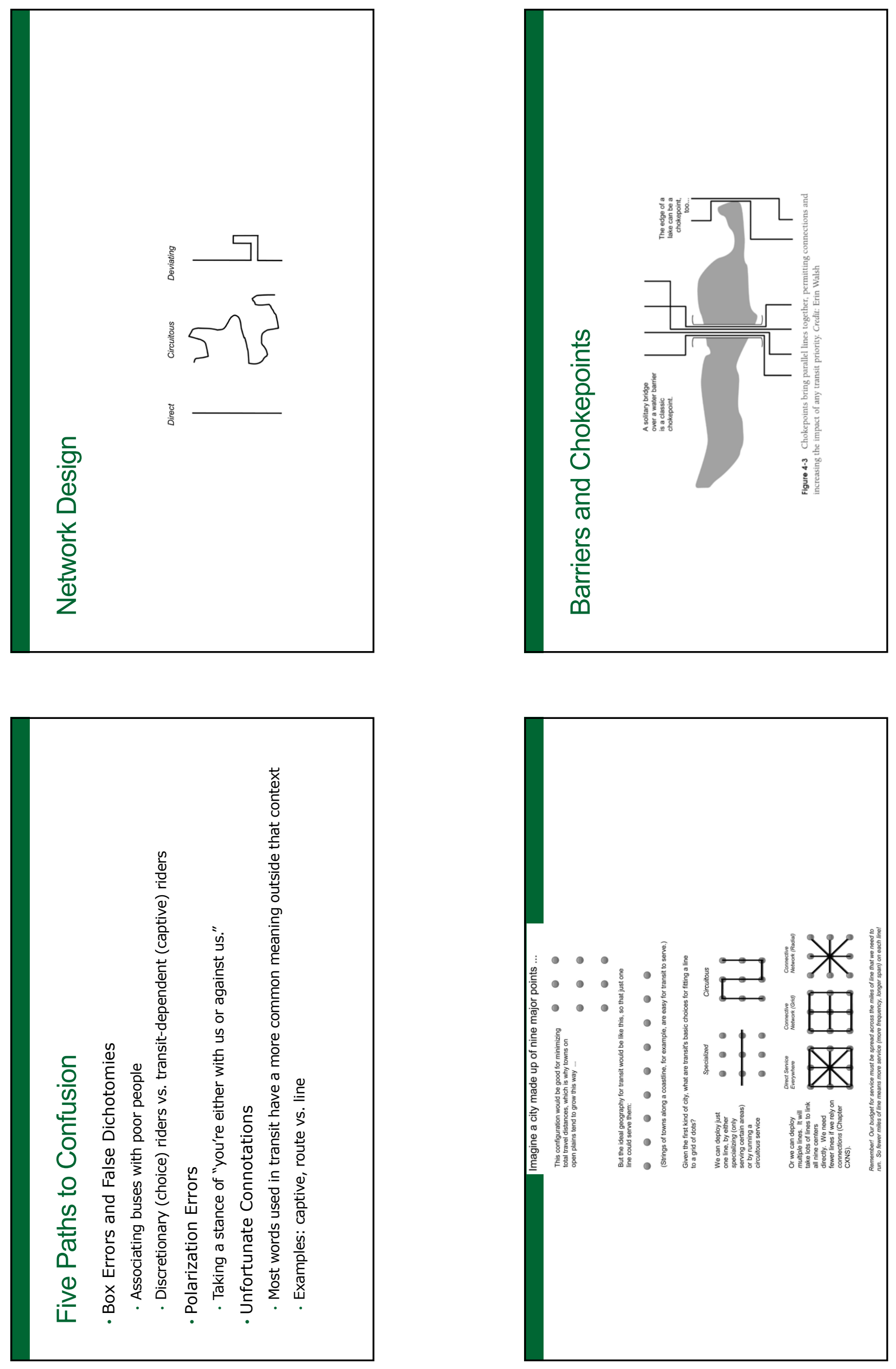

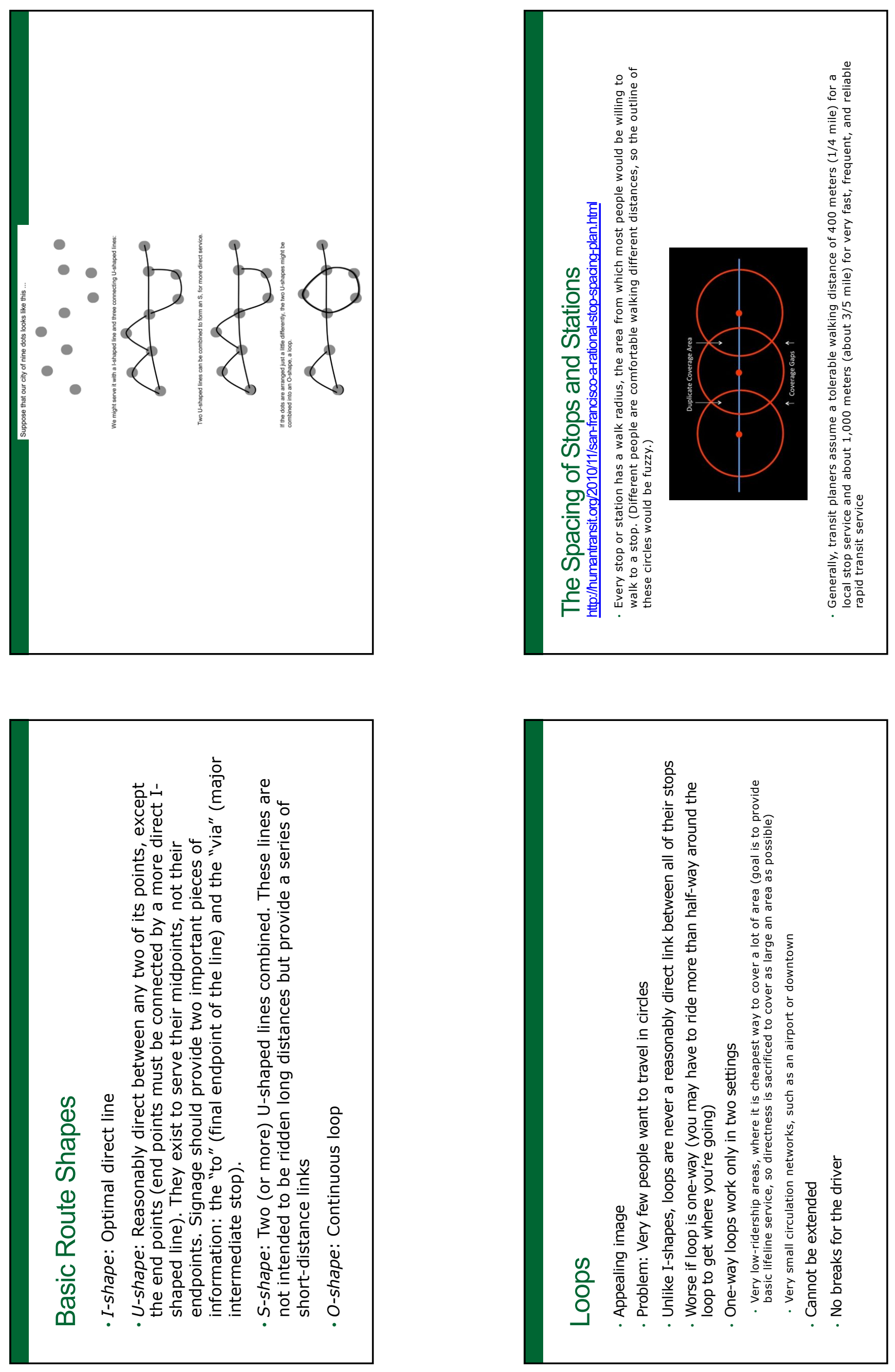

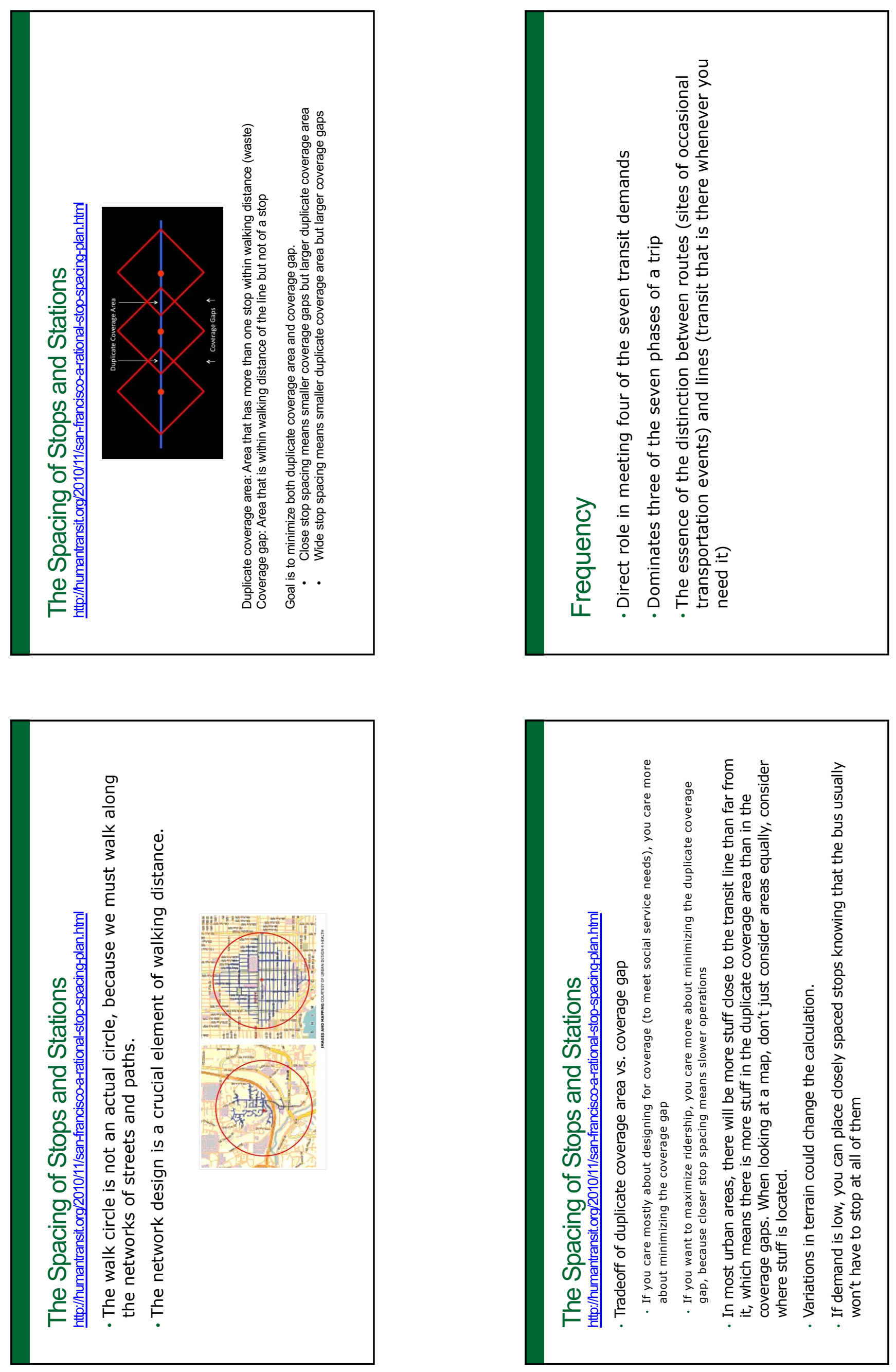

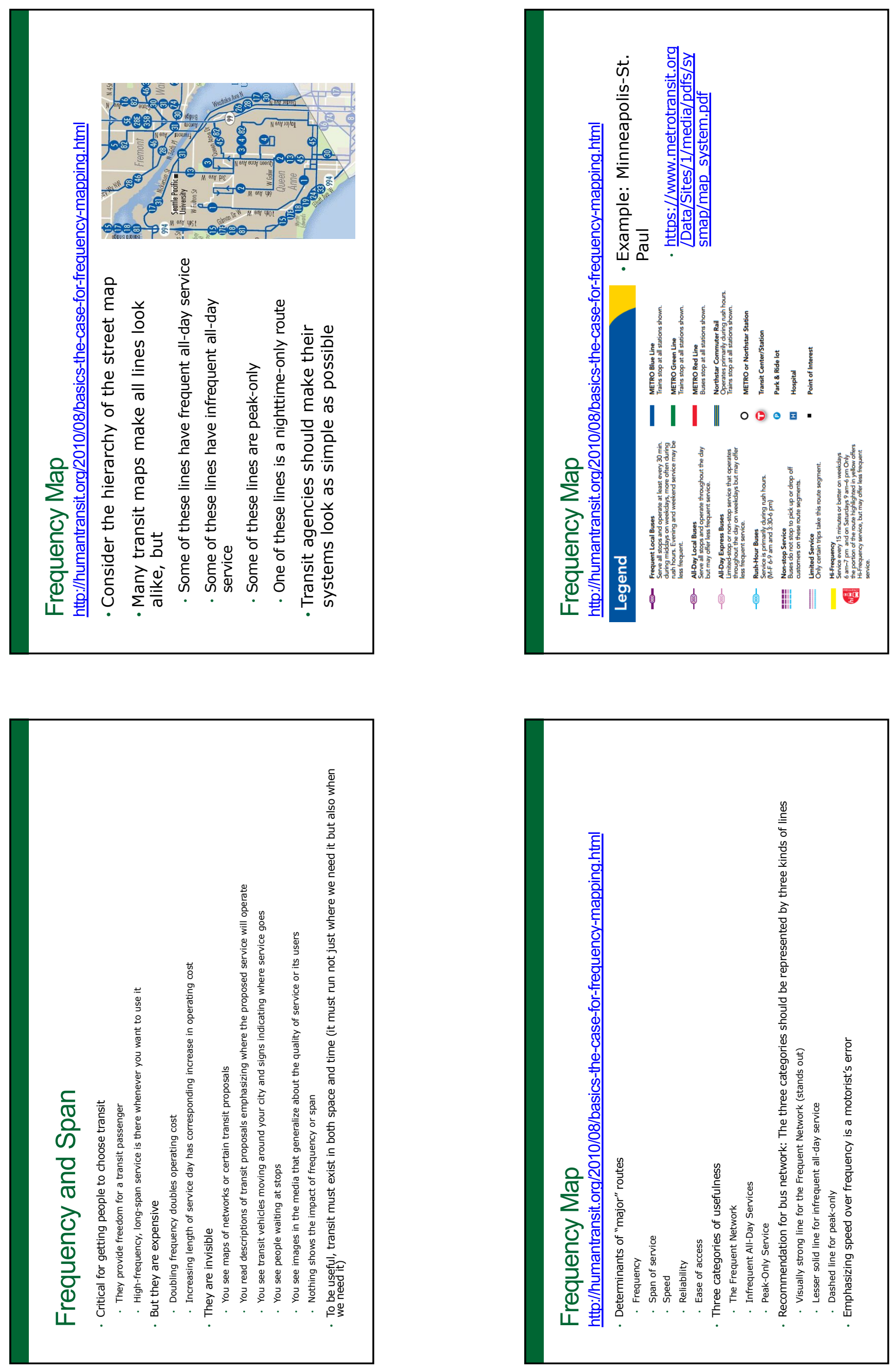

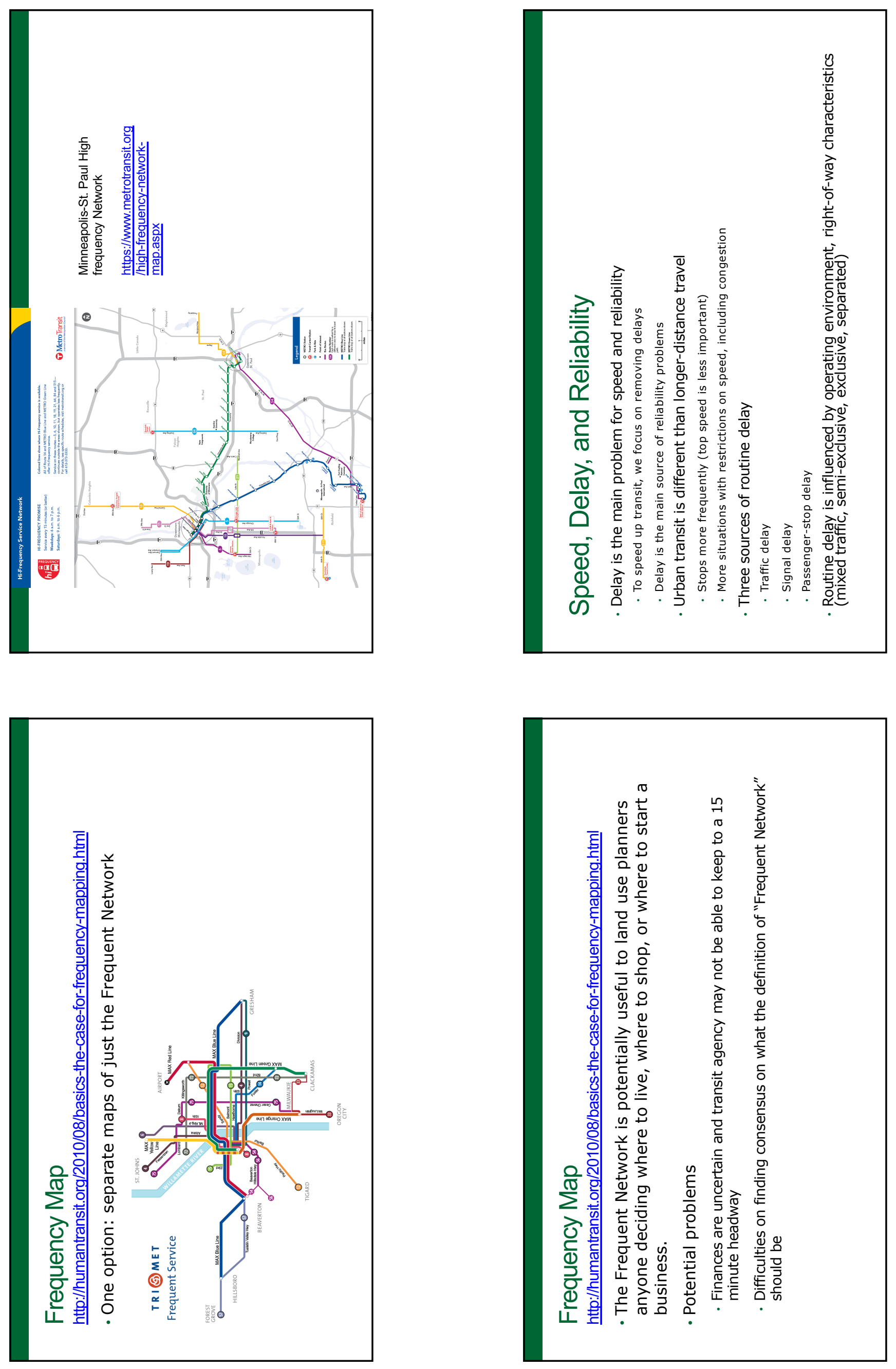

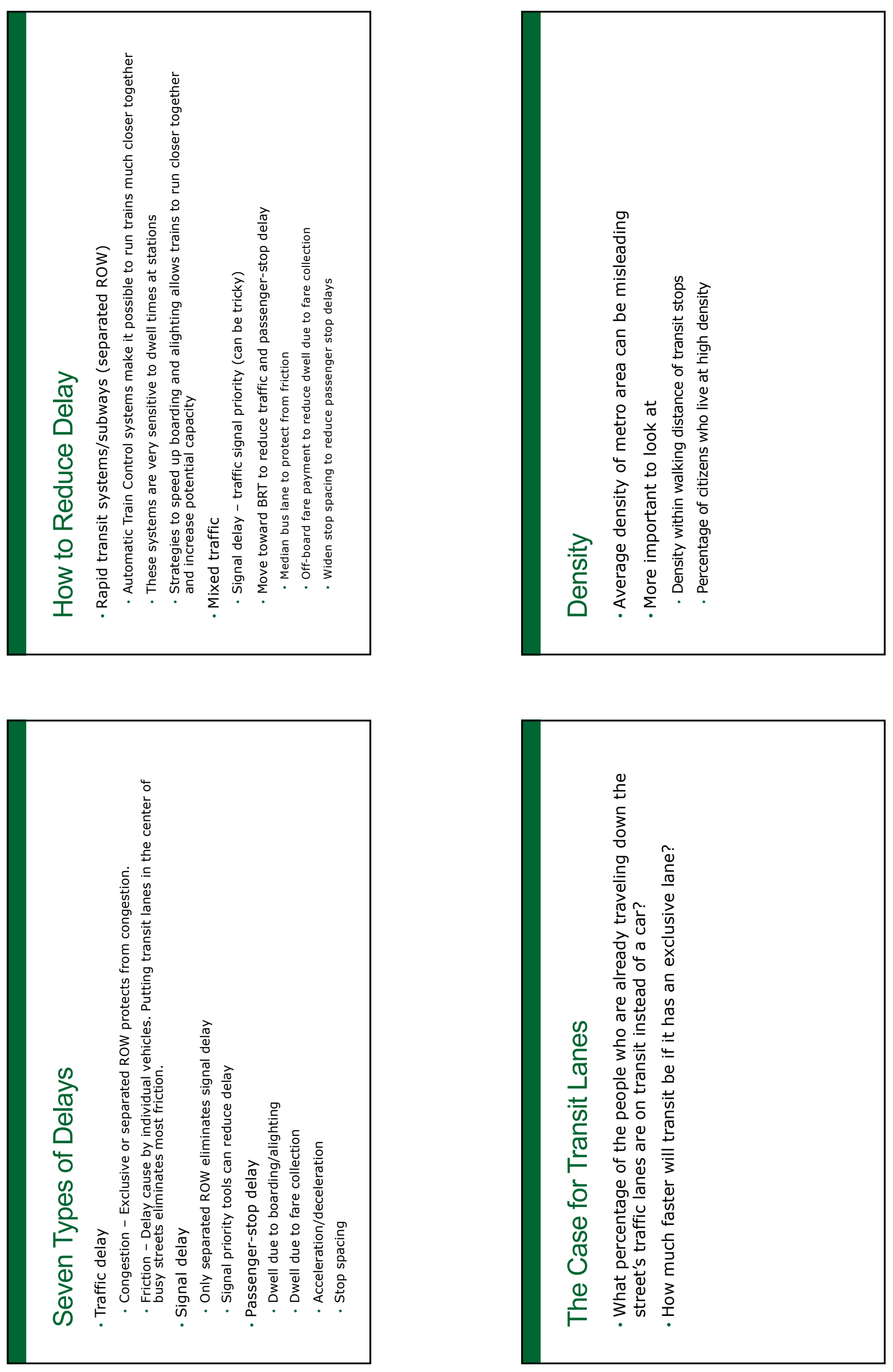

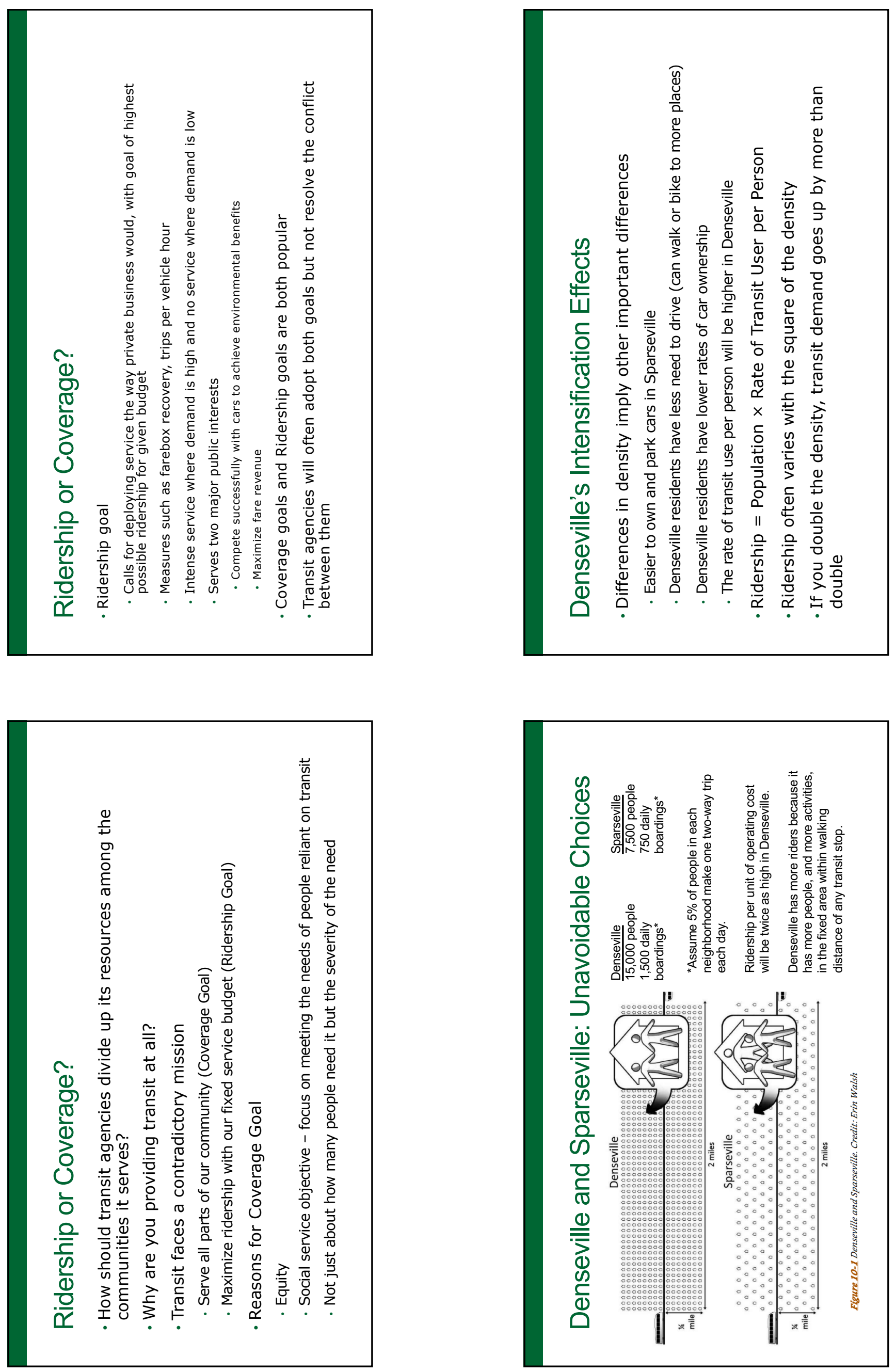

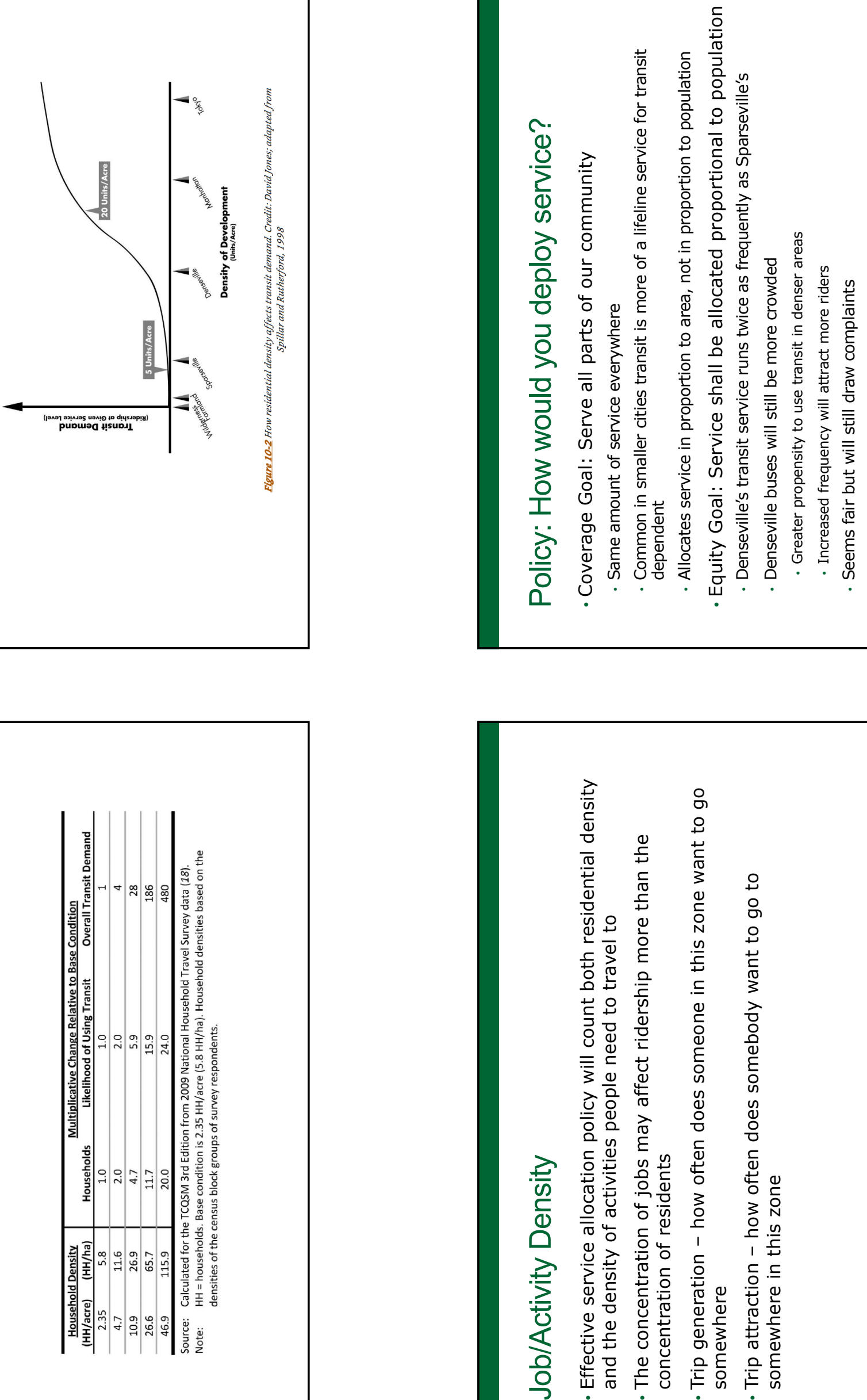

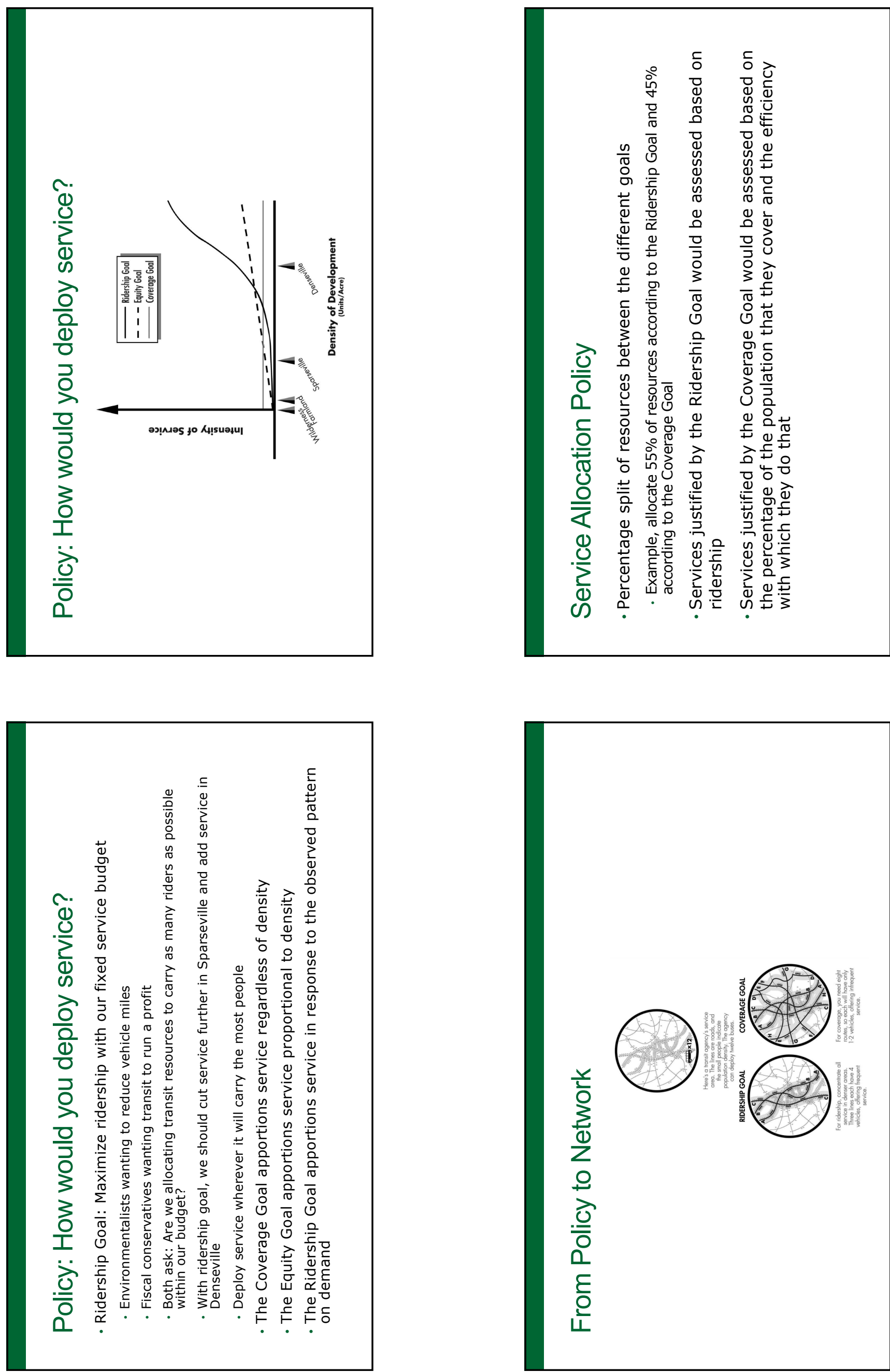

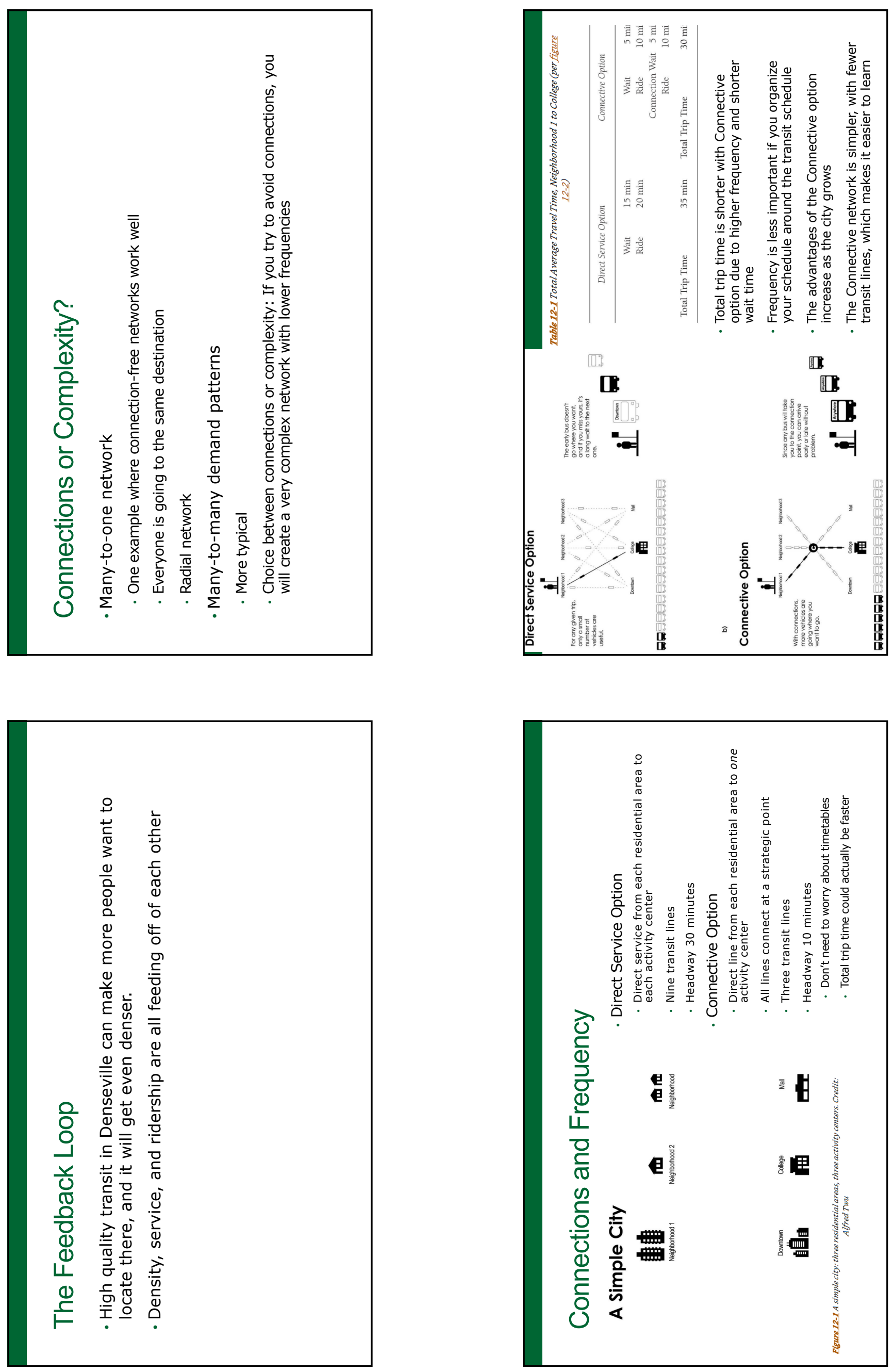

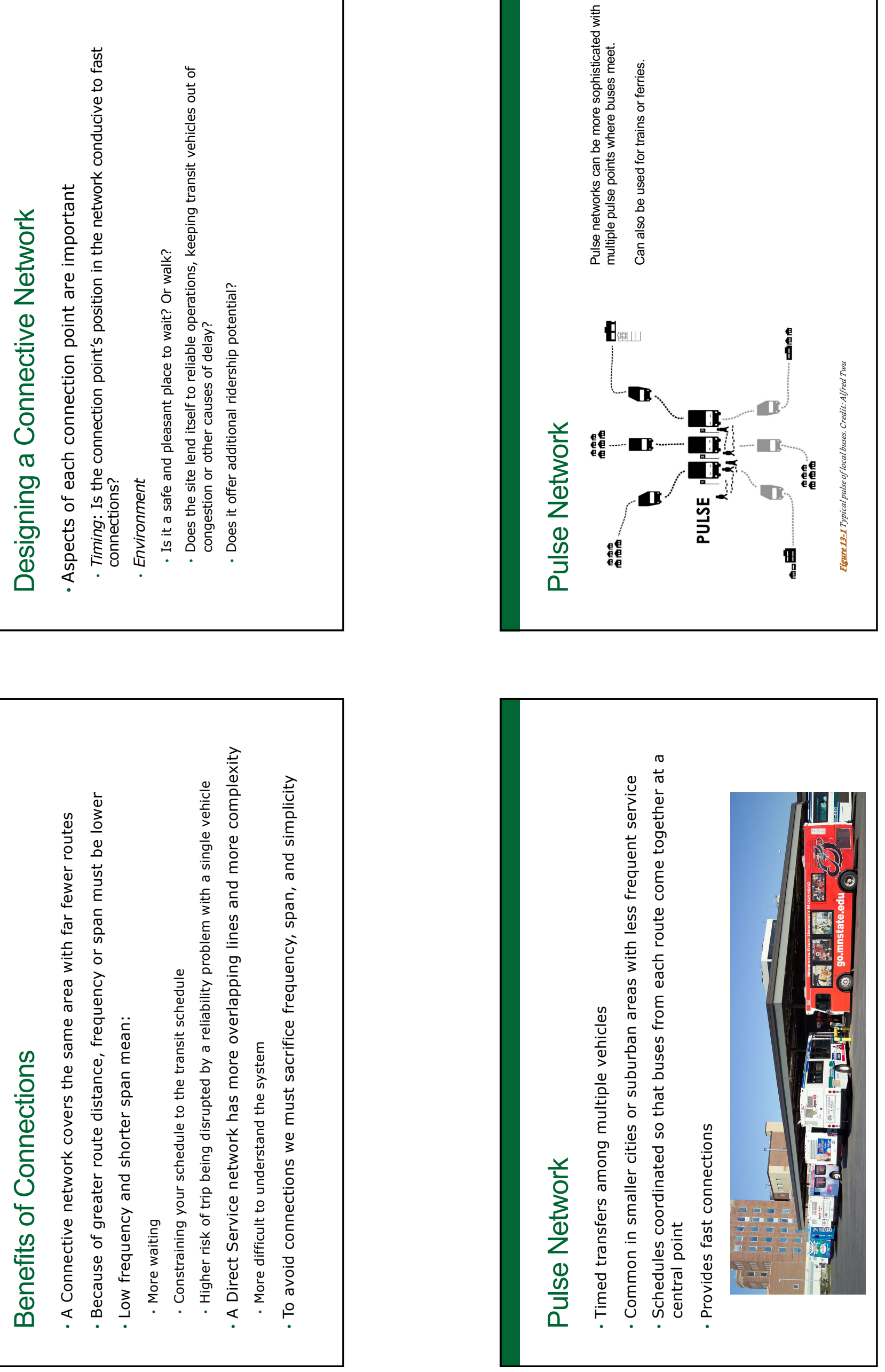

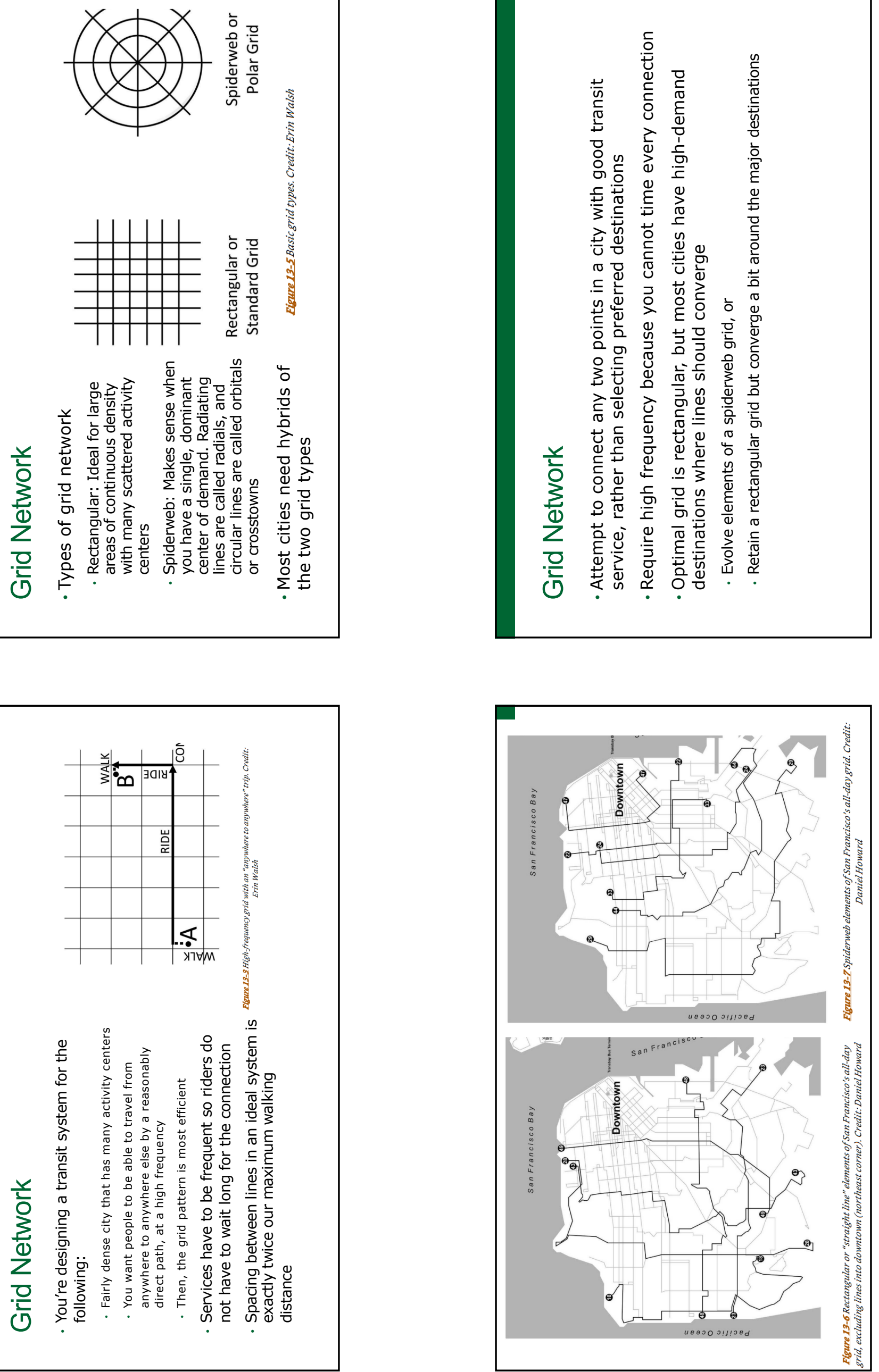

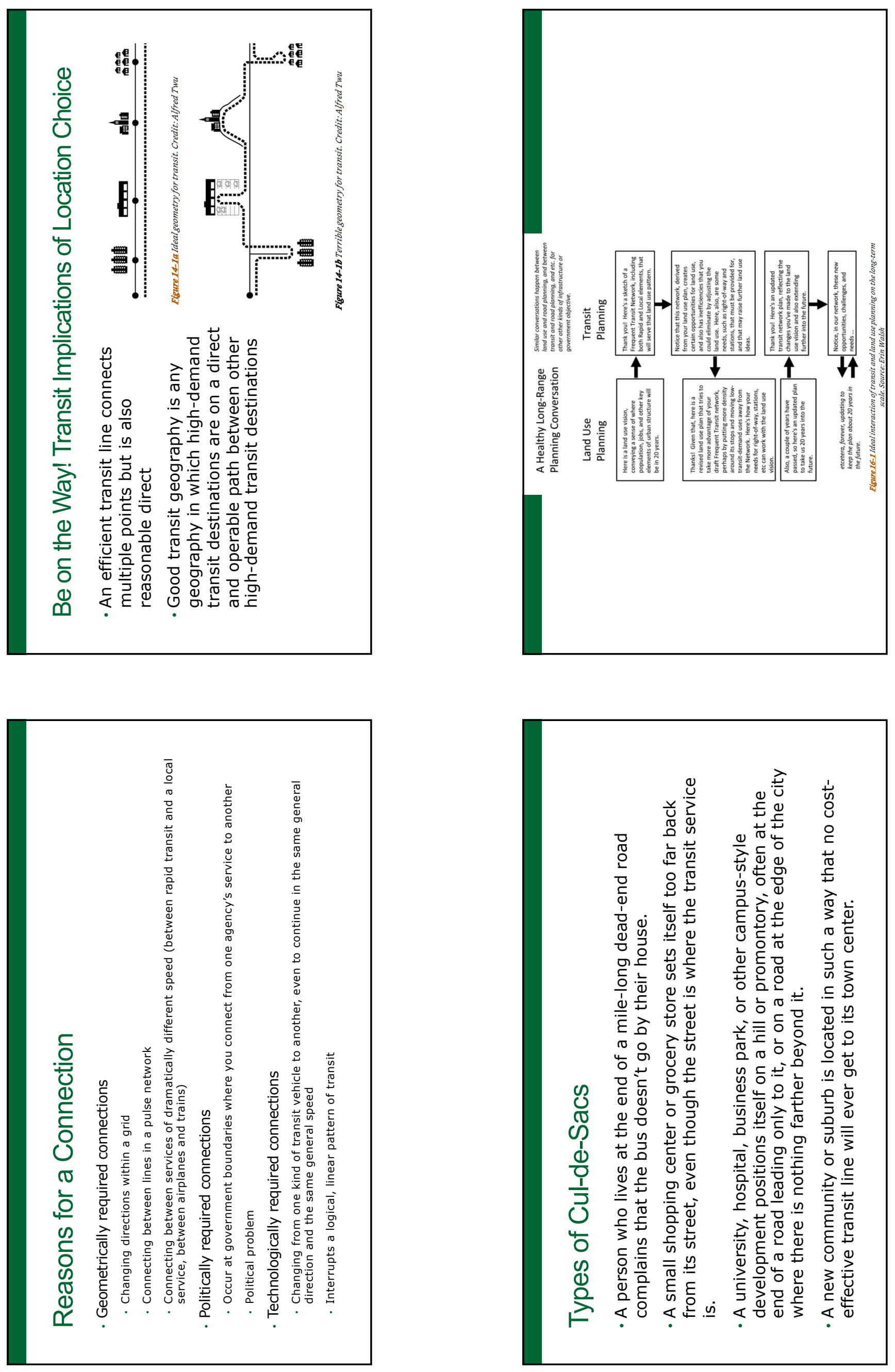

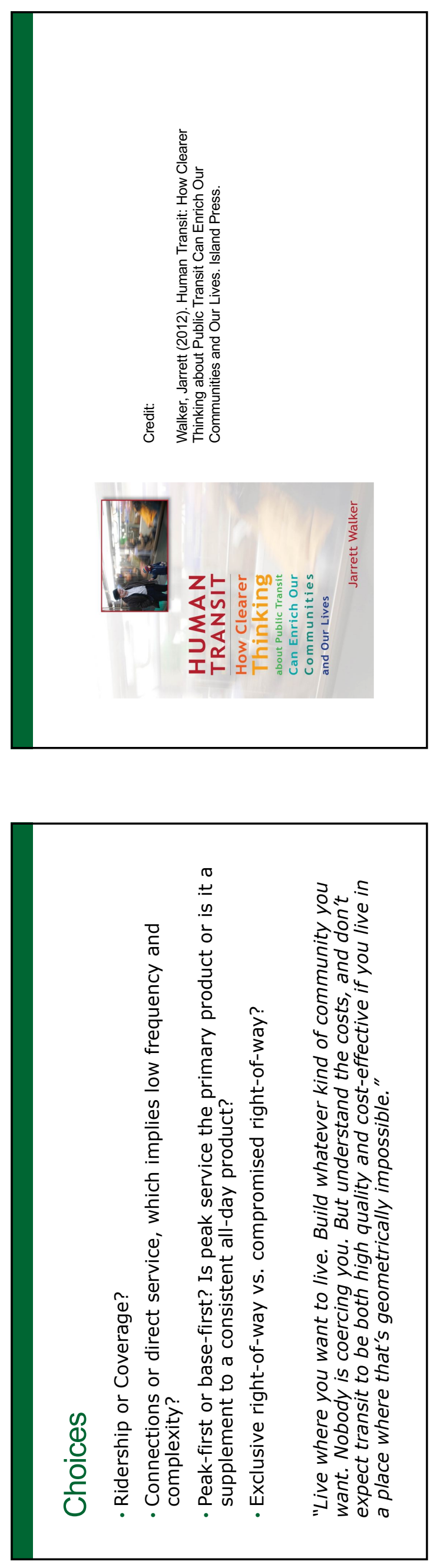

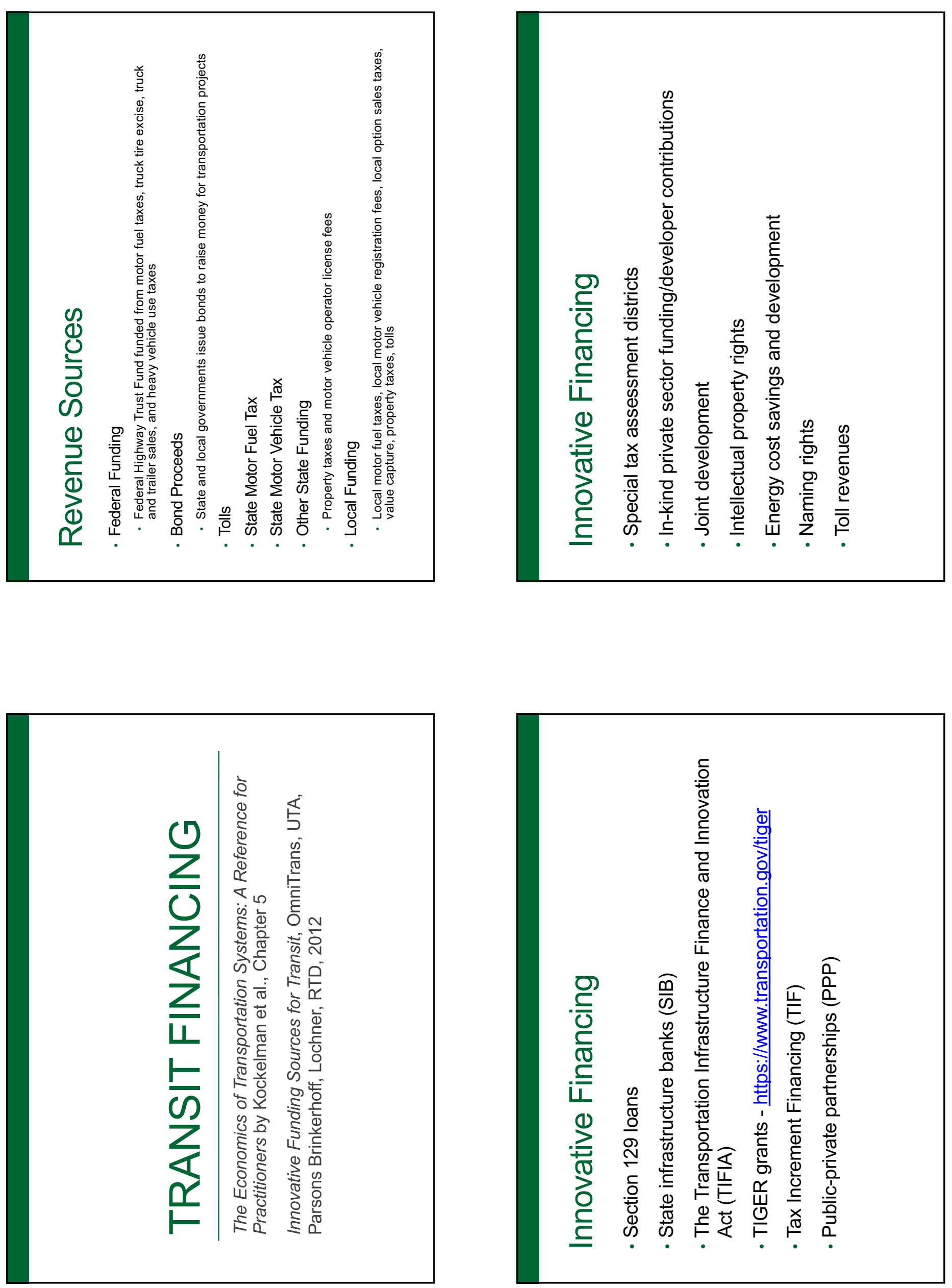

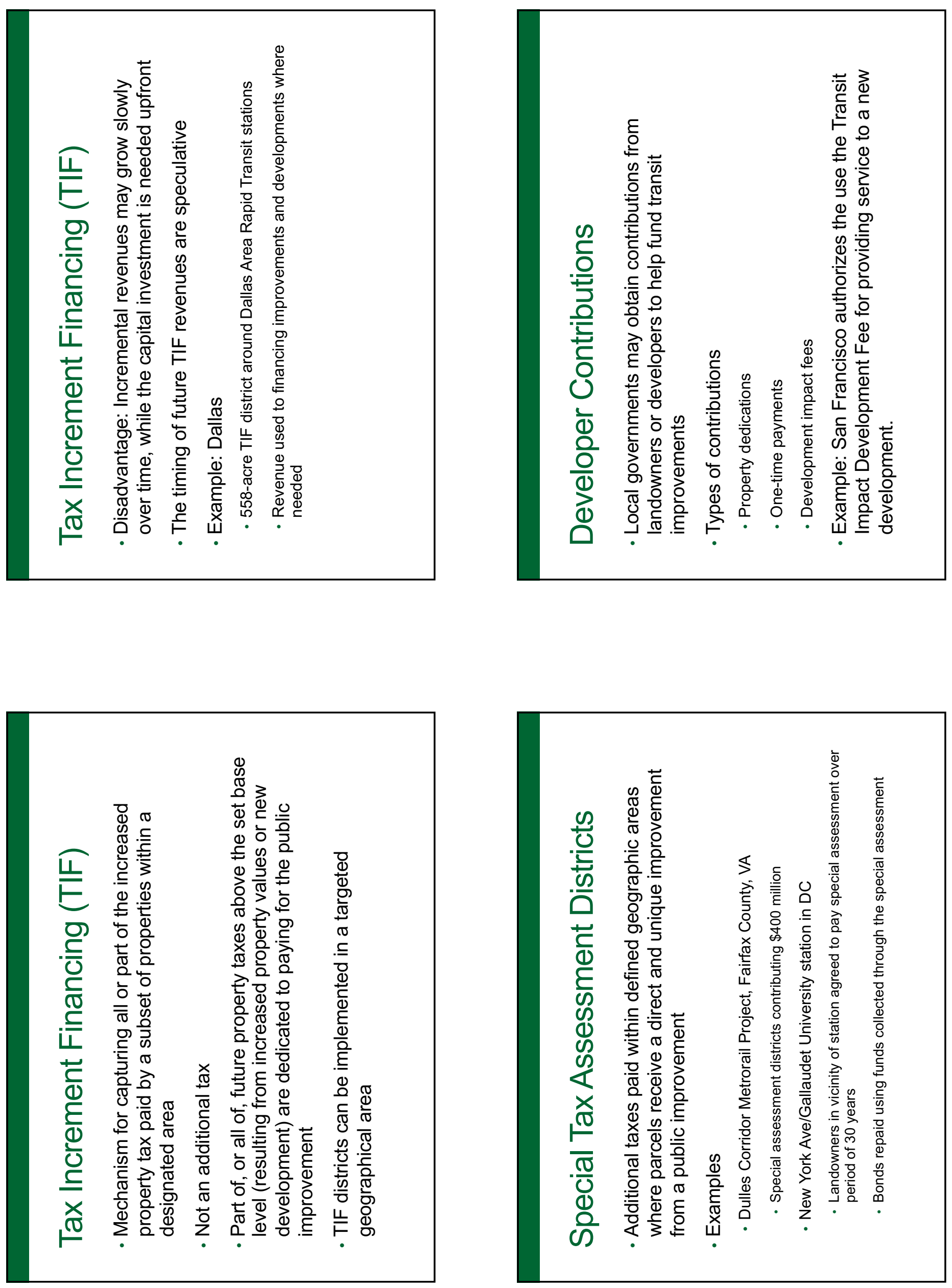

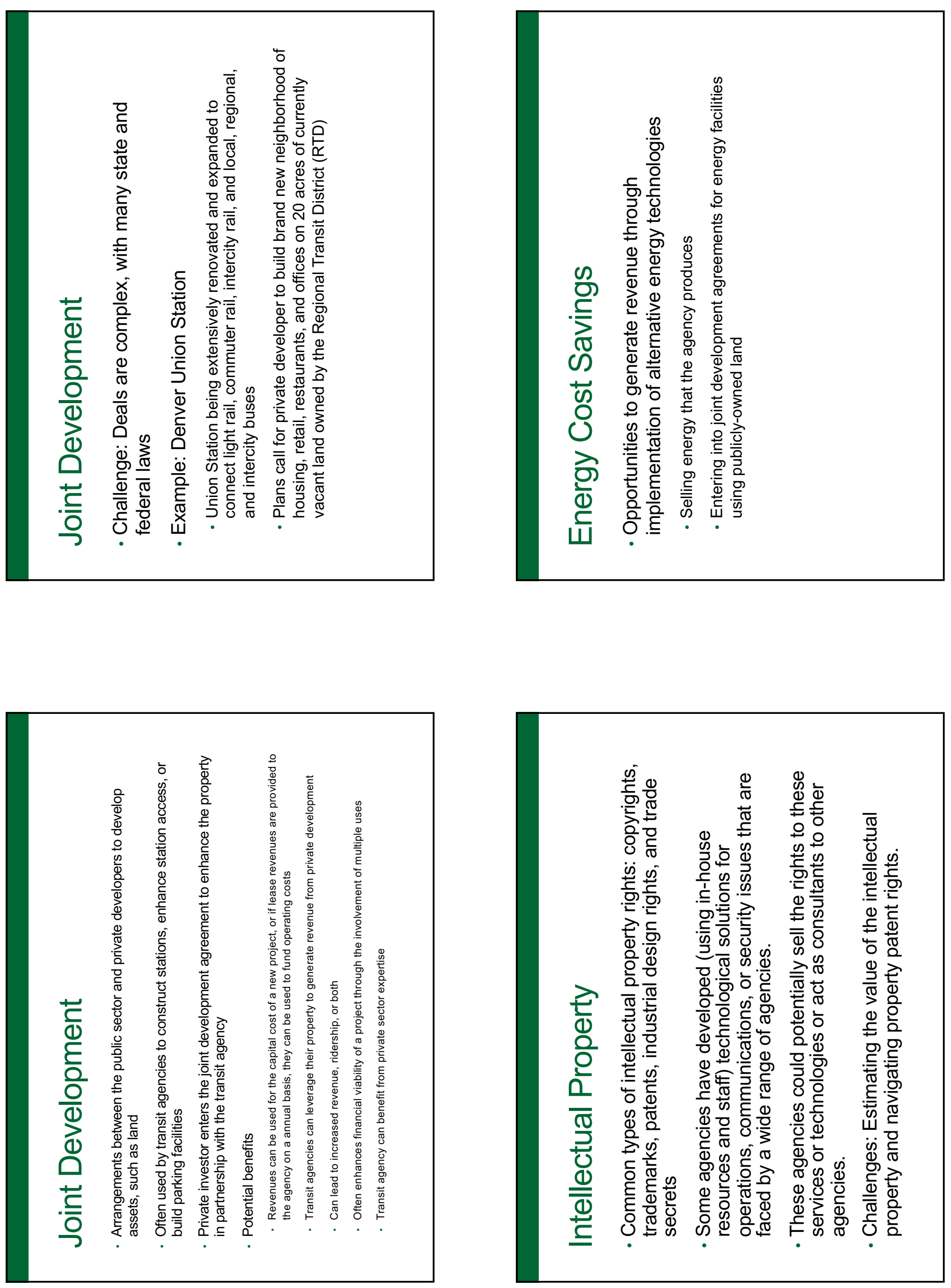

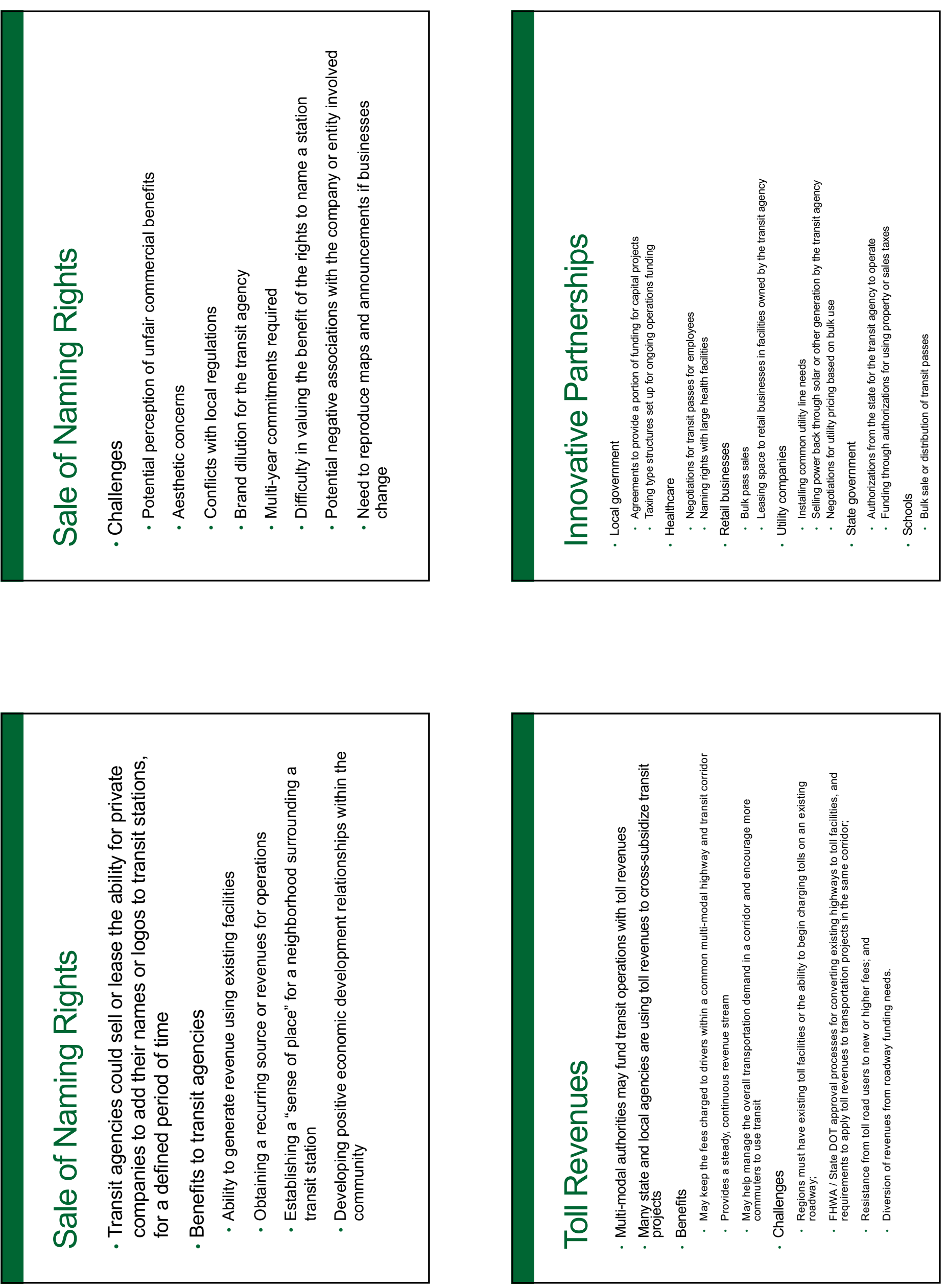

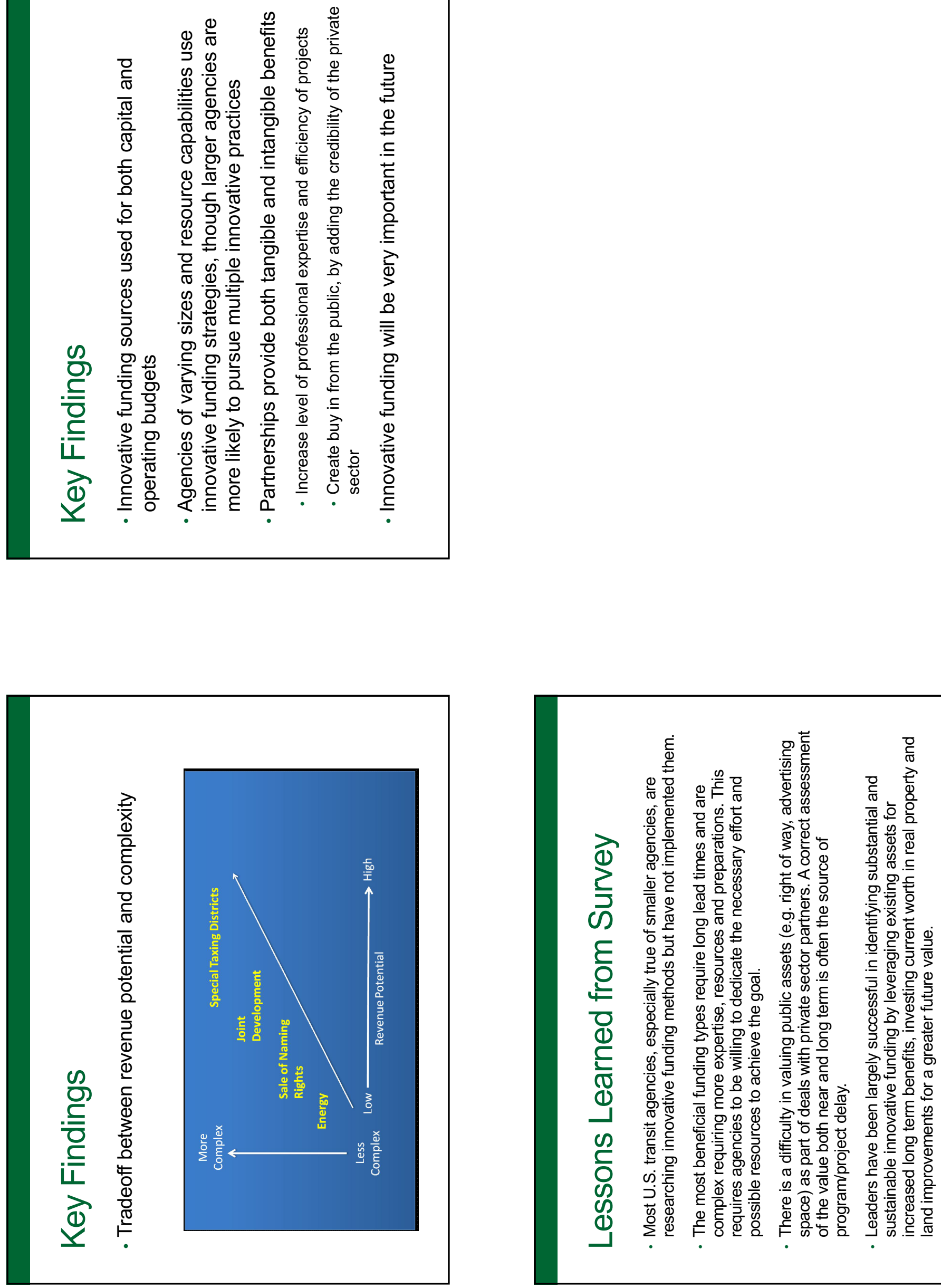

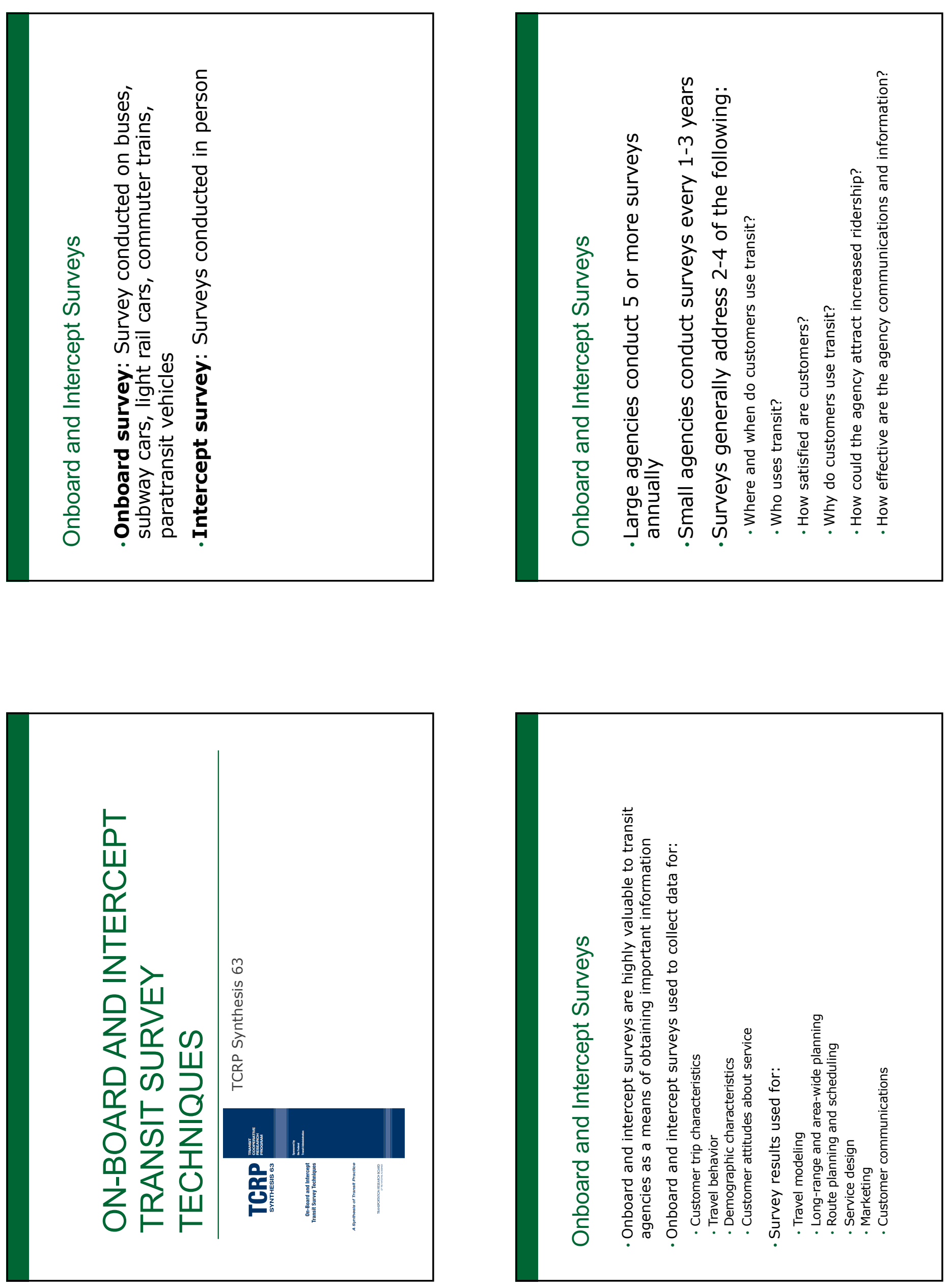

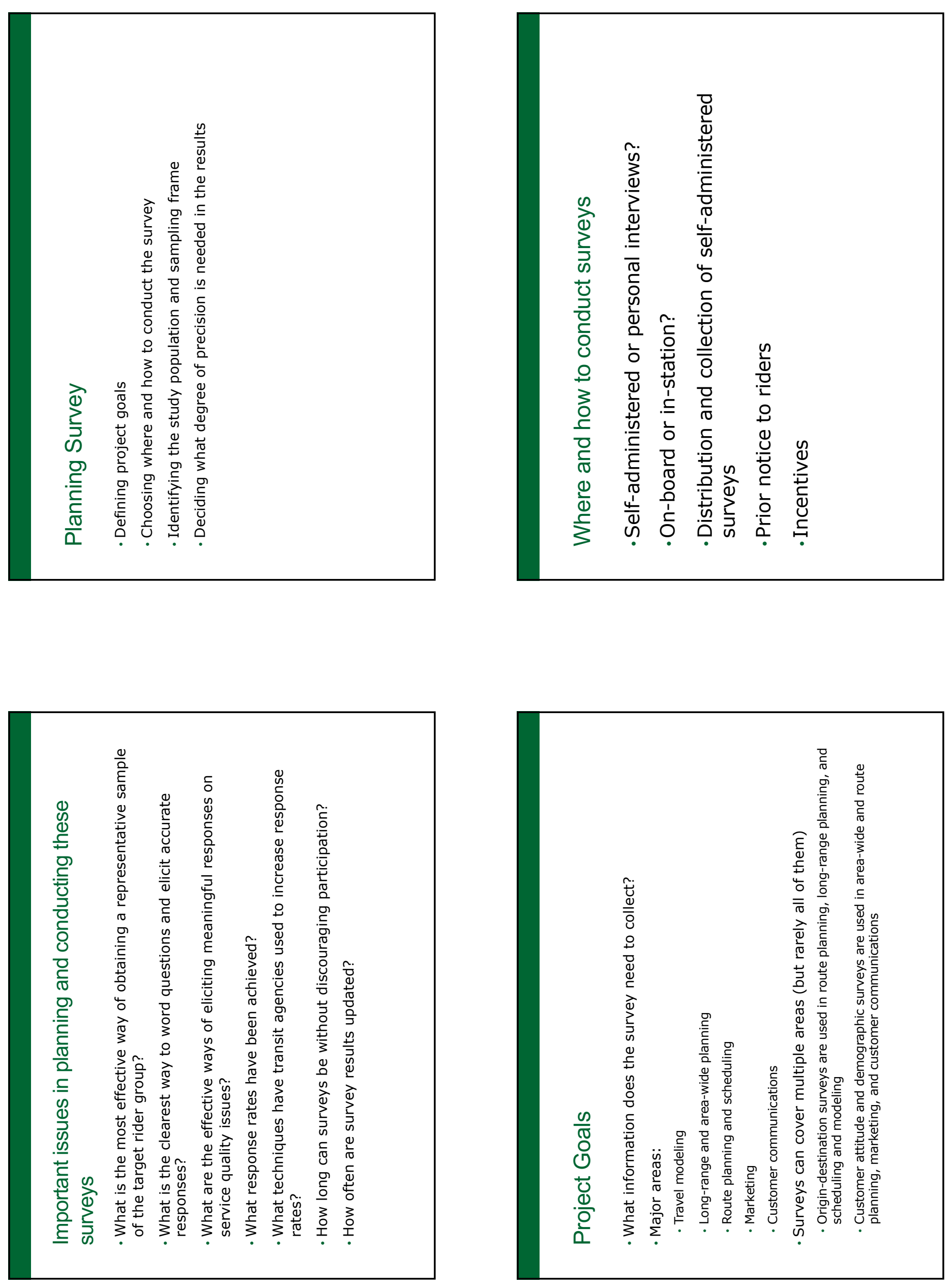

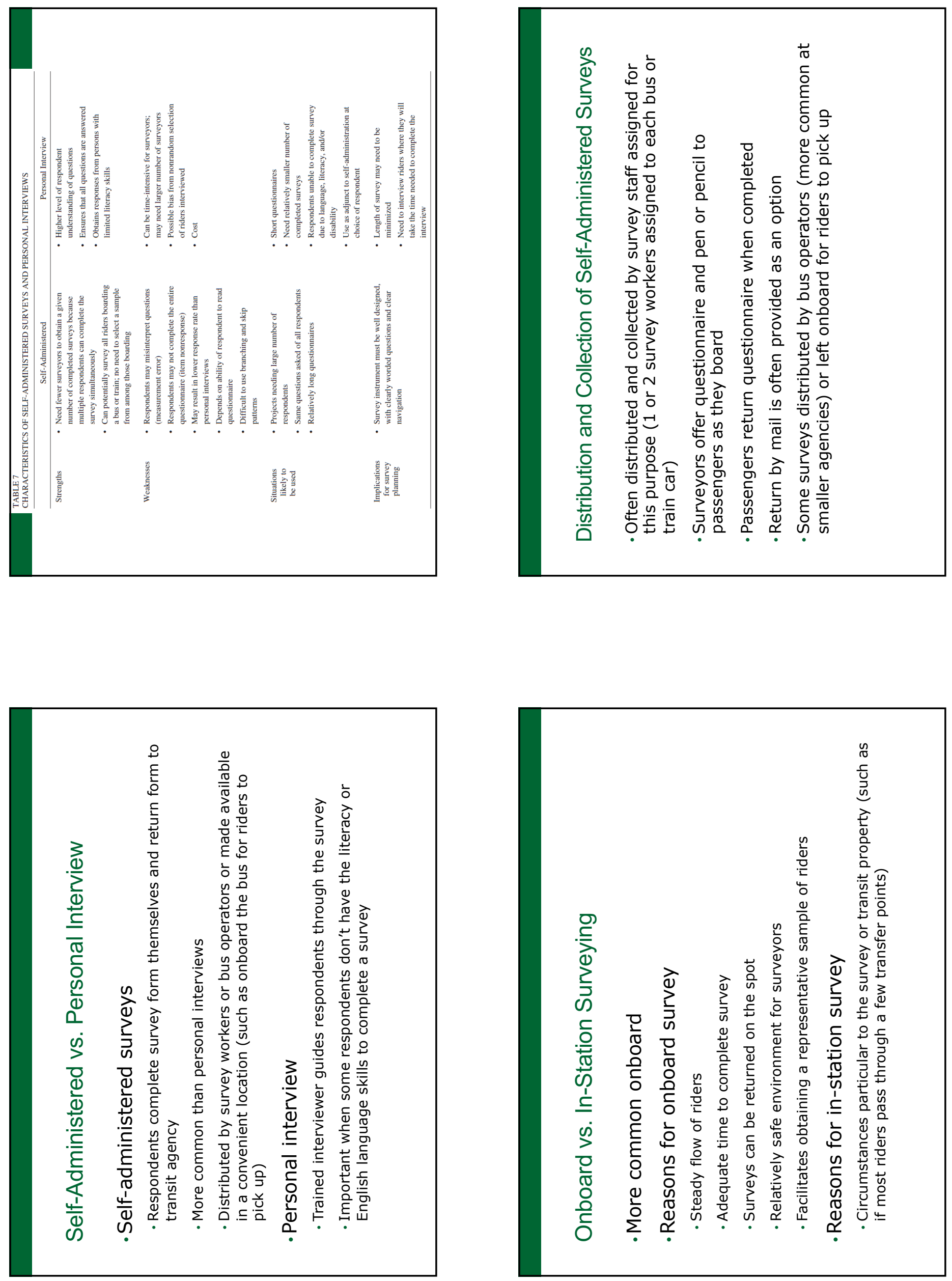

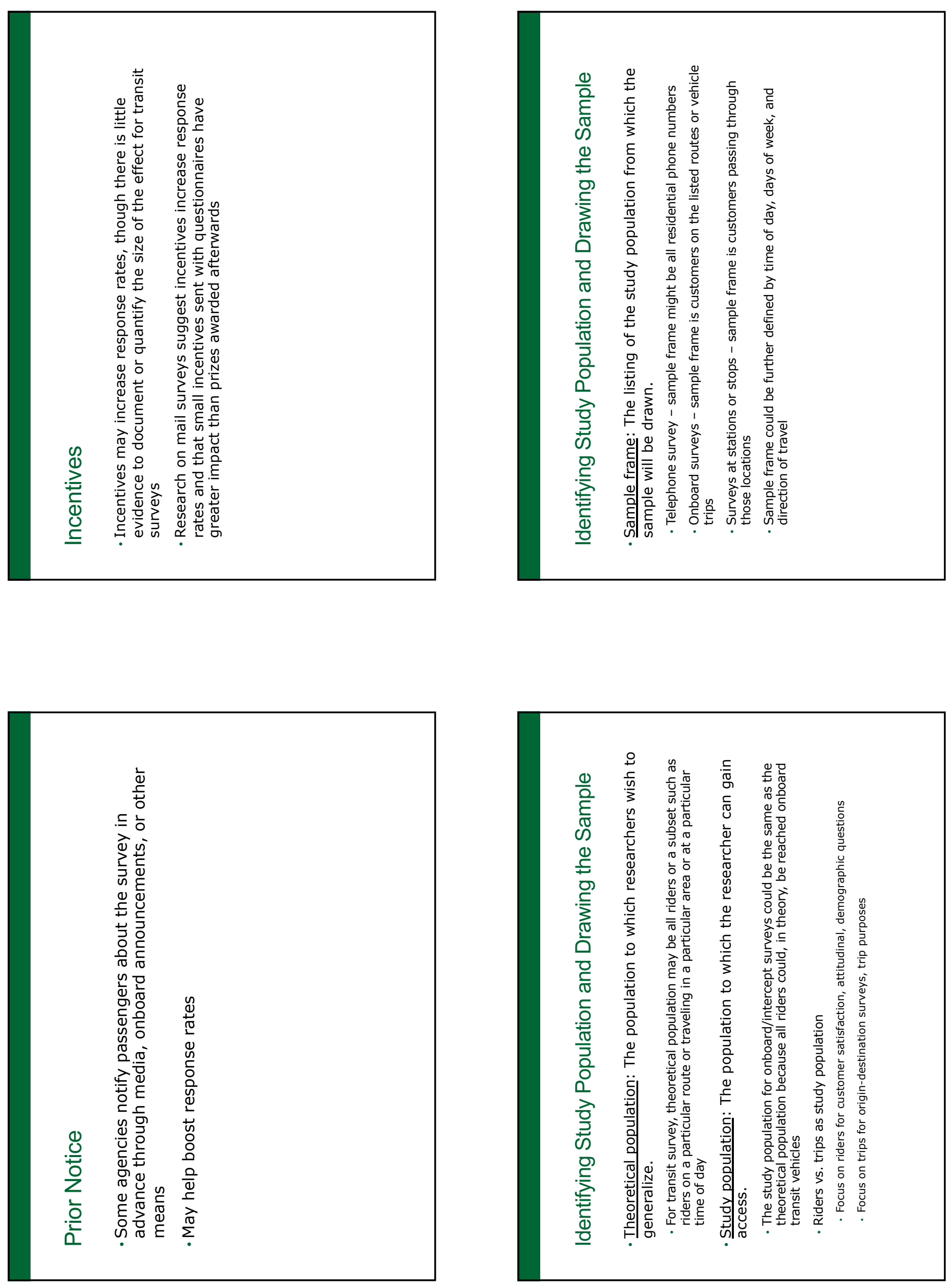

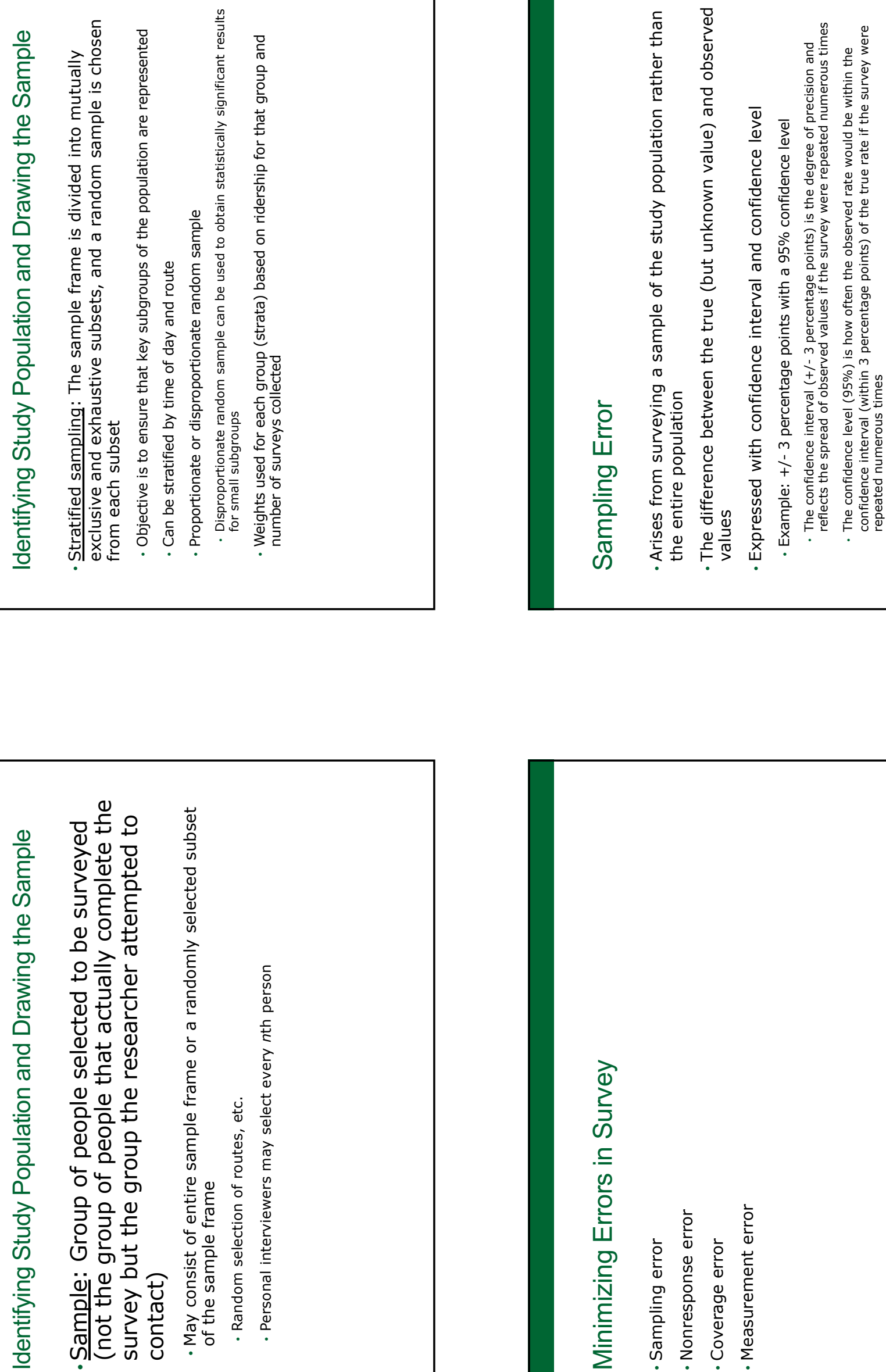

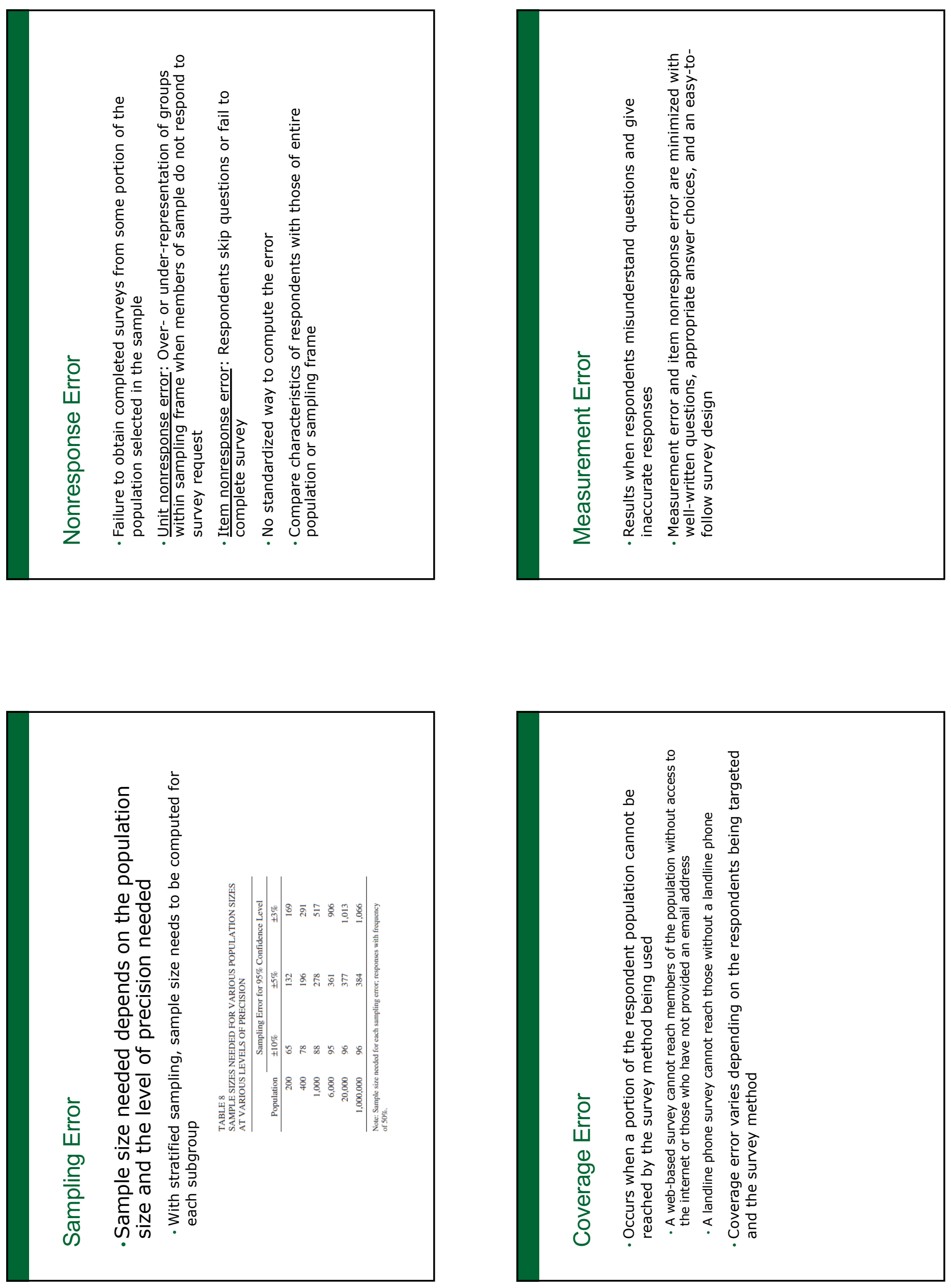

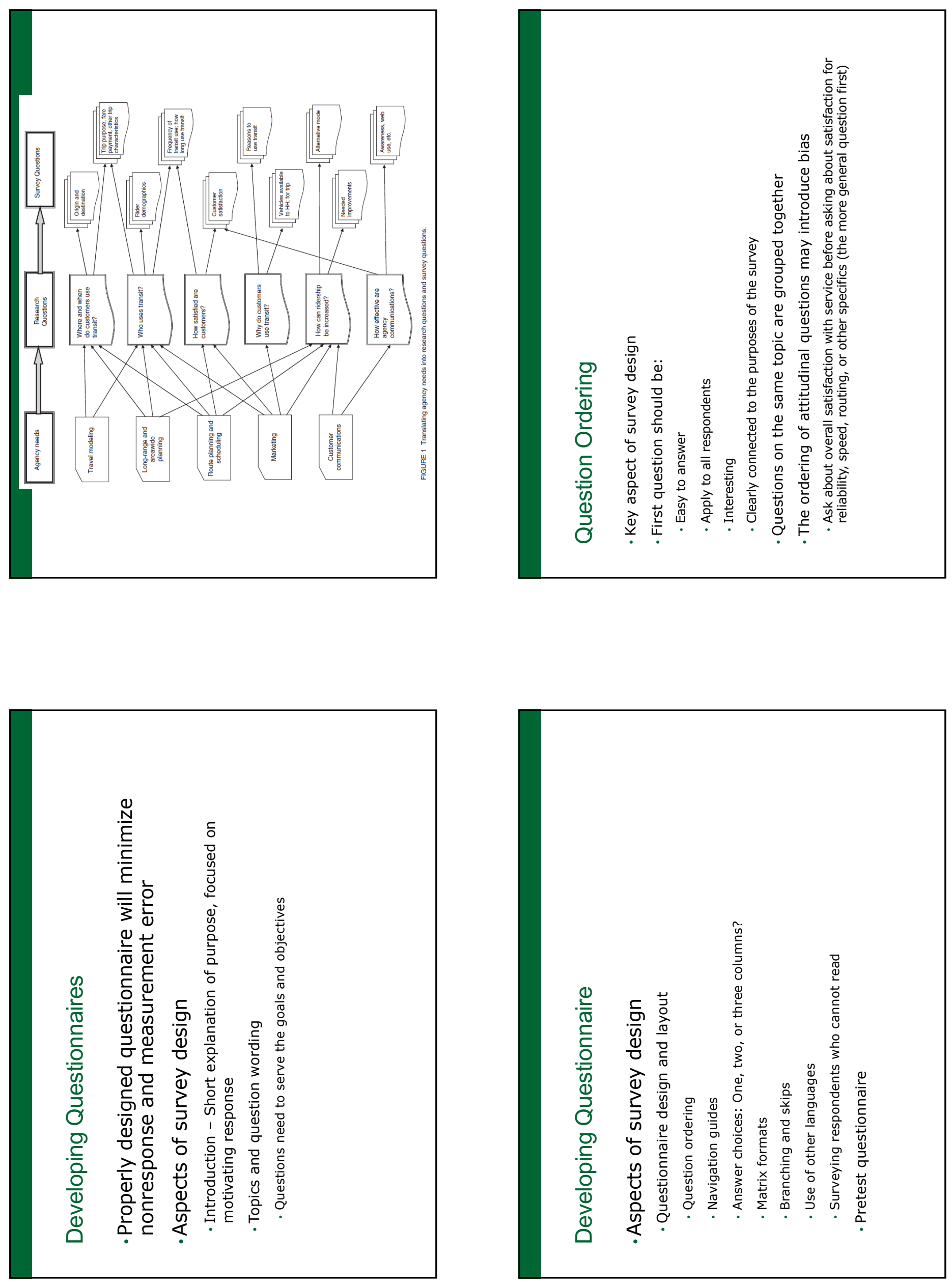

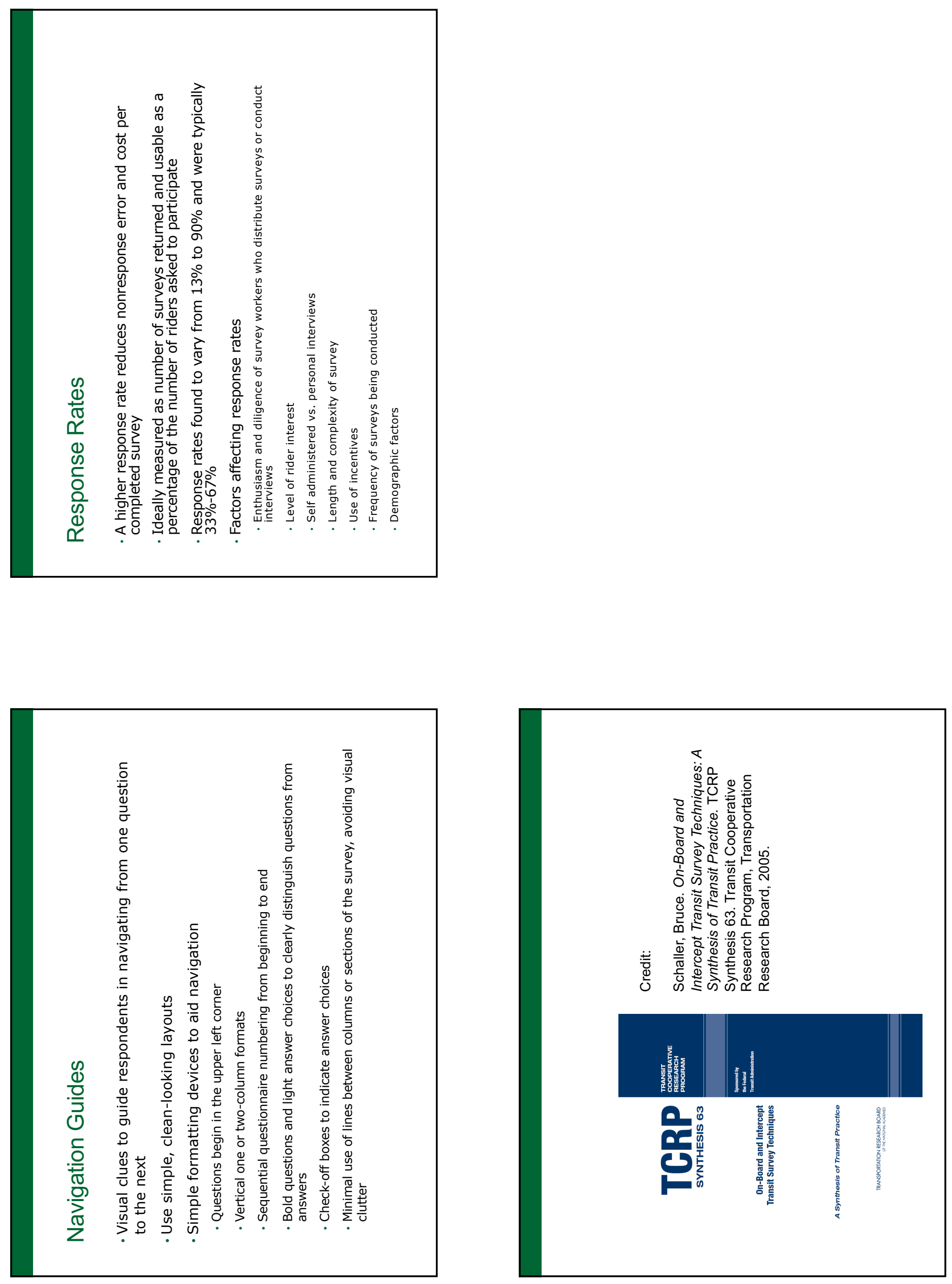
ASSIGNMENTS 


\section{TL 787}

\section{Homework 1}

Due: January 22

1. What is your background knowledge, interest, and experience with transit, and what do you hope to learn from this course?

2. Describe the transit system in your home community. (If none exists, describe the transit system of another city.) Describe the modes available, types of vehicles, operating environments, system design, etc. 


\section{TL 787}

\section{Homework $2^{1}$}

\section{Due: February 1}

The objective of this assignment is to make use of the expertise of transit planning, design and operations experts from around the world from the Transportation Research Board annual meeting.

You should choose a paper written for a recent TRB annual meeting to write an approximately one page summary. The essay should be organized in the following five paragraph structure:

1. Introduction (including thesis statement)

2. Body Paragraph One (strongest argument/piece of evidence/theme)

3. Body Paragraph Two (additional argument/piece of evidence/theme)

4. Body Paragraph Three (additional argument/piece of evidence/theme)

5. Conclusion (ties together, summarizes, broader perspective)

You can search for papers at http://amonline.trb.org/. If you do not have free access to the full papers, you may email the instructor with the requested paper number and it will be emailed to you.

You should choose a paper with a focus on topics related to public transportation. In general, this would be sessions sponsored by:

- AL020 Transit and Intermodal Transportation Law

- AP010 Transit Management and Performance

- AP015 Transit Capacity and Quality of Service

- AP020 Emerging and Innovative Public Transport and Technologies

- AP025 Public Transportation Planning and Development

- AP030 Public Transportation Marketing and Fare Policy

- AP040 Major Activity Center Circulation Systems

- AP045 Intermodal Transfer Facilities

- AP050 Bus Transit Systems

- AP055 Rural Public and Intercity Bus Transportation

- AP060 Paratransit

- AP065 Rail Transit Systems

- AP070 Commuter Rail Transportation

- AP075 Light Rail Transit

- AP085 Ferry Transportation

- AR055 Rail Transit Infrastructure

- Some papers in ABE30 Transportation Issues in Major U.S. Cities

- Some papers in ABE60 Accessible Transportation and Mobility

\footnotetext{
${ }^{1}$ This assignment was developed by the Southeastern Transportation Research, Innovation, Development and Education Center (STRIDE). STRIDE's Public Transportation Course Modules and associated assignments are available at https://stride.ce.ufl.edu/2017/04/public-transportation-course-modules/
} 


\section{TL 787}

\section{Homework $3^{1}$}

\section{Due: February 8}

1. (7 points) A heavily traveled bus line has a length of $12 \mathrm{~km}$. It has stops every intersection and there are 5 blocks per kilometer (a bus stop every 200 meters). The running speed of the buses is $34 \mathrm{~km} / \mathrm{h}$. Terminal time is 9 minutes at each end. Assume that the buses stop at every stop, losing 20 seconds for deceleration and acceleration plus dwell time of 15 seconds every stop. The average passenger trip length on the line is $4.8 \mathrm{~km}$. Service headway is 5 minutes.

You want to propose a reduction of stop density to one stop per two blocks. In that case, time loss for deceleration/acceleration per stop would not change, but dwell times would increase to 25 seconds per stop due to an increased number of passengers boarding and alighting per stop. The company management believes that the existing operation is desirable for passengers (short access) and that the changes would not make much of a difference in operating costs anyway. To convince management that the change would be useful, compute and systematically present the following consequences of the change in stop density, assuming uniform distribution of origins-destinations along the whole line:

a. Additional average walking distance per passenger along the line, which includes access and egress (in meters per person)

b. Additional average access and egress time (in minutes per person) if the speed of walking is 75 meters per minute.

c. Reduction of the average passenger travel time on buses (in minutes per person).

d. Change in passenger total travel time.

e. How many buses can be saved due to higher operating speed? HINT: calculate roundtrip cycle time under current and proposed scenario and calculate number of buses needed based on cycle time and headway.

2. (7 points) A $14-\mathrm{km}$ (one-way) long trolleybus line has an operating speed $V_{0}=12 \mathrm{~km} / \mathrm{h}$; terminal time at each end $t_{t}$ is at least 6 minutes.

a. How many trolleybuses are required for operation with a 10-minute headway? What will be the cycle speed $V_{c}$ for the line with that schedule?

b. What new operating speed $\mathrm{V}^{\prime}$ o should be achieved in order to reduce the number of trolleybuses by two, while maintaining the same headway and increasing the minimum required terminal time at one terminal to 8 minutes.

c. What will be the offered line capacity if $C_{v}=80$ spaces? Compute $\alpha_{\max }$ if the line carries 300 persons per hour on its maximum load segment?

\footnotetext{
${ }^{1}$ Source of homework problems: Vuchic, Vukan R. (2005) Urban Transit Operations, Planning, and Economics. Hoboken, New Jersey: John Wiley \& Sons, Inc.
} 
3. (6 points) A rapid transit line is $\mathbf{1 4 . 2} \mathrm{km}$ long (one way) and has the following passenger volumes boarding and alighting at individual stations in the peak direction during the peak hour.

\begin{tabular}{|l|c|c|c|c|c|c|c|c|c|c|c|}
\hline Station & A & B & C & D & E & F & G & H & I & J & K \\
\hline $\begin{array}{l}\text { Boardings } \\
\text { (prs/h) }\end{array}$ & 3300 & 1700 & 1900 & 3200 & 2900 & 1300 & 1600 & 600 & 400 & 700 & 0 \\
\hline $\begin{array}{l}\text { Alightings } \\
\text { (prs/h) }\end{array}$ & 0 & 0 & 500 & 700 & 2100 & 700 & 4000 & 2700 & 2200 & 1700 & 3000 \\
\hline
\end{tabular}

Capacity of each train is 590 spaces, $\alpha_{\max }$ is selected to be 0.9 , travel speed on the line is $V_{0}=36$ $\mathrm{km} / \mathrm{h}$. Terminal time is 5 minutes at each terminal.

a. Compute the maximum load segment (MLS)

b. Compute the headway, frequency, number of trains required for the service, actual load factor, cycle time, and cycle speed. 


\section{TL 787}

\section{Homework 4}

\section{Due: February 15}

1. (5 points) Describe the concept of elasticities, including different types of elasticities, and how elasticities can vary.

2. (5 points) Given a calibrated utility function, $u=b-0.04 C-0.02 t$, where $C$ is the cost of travel (in cents) and $t$ is the travel time (in minutes).

a. What will be the modal split (percentage demand traveling by each mode) for the data given below?

b. Given rising gasoline prices, if $C$ for a car increases by $\$ 1.20$ (from 110 cents to 230 cents), what impact will this change have on the modal split?

\begin{tabular}{llll}
\hline Mode & $b$ & $C$ & $t$ \\
\hline Bus & -0.30 & 85 & 30 \\
Light rail & -0.35 & 100 & 50 \\
Car & -0.25 & 110 & 35 \\
\hline
\end{tabular}

3. (5 points) Pick a rural county (a county with no metro area) located in your home state. Estimate demand for general purpose rural public transit in the county. Use the equation from TCRP 161:

Trips per year $=(2.20 *$ Populated age 60 or older $)+(5.21 *$ Mobility Limited Population age 18 to 64$)+(1.52 *$ Residents of Households having No Vehicle)

Use 2014 ACS 5-year data, which can be accessed through the American FactFinder. Use table numbers indicated in the lecture.

4. (5 points) Find a news article about ridership for a particular transit agency. (It doesn't have to be a recent article, but you should be able to find several articles if you do a Google news search.) Post the article on the discussion board in the Demand Analysis thread, and then give a one paragraph summary on the discussion board. In your summary, you should 1) briefly describe changes in ridership, 2) summarize the factor(s) believed to be contributing to the ridership changes, 3 ) identify if these factors are internal factors or external factors, and 4) discuss whether you think the given explanations for ridership changes are reasonable. 


\section{TL 787}

\section{Homework 5}

\section{Due: February 26}

1. In week 4 lecture, we have learned about transit trip decision making process from availability, and comfort and convenience stand point. In this context, please explain what mode you mostly chose to travel while making your regular weekday trips to work/school. Do you use public transit in making your every day trips? Please provide your explanation about your trip decision making process about why you do/do not choose transit for your regular trips. You can explain your answer from availability, and comfort and convenience stand point.

2. The objective of this task is to get a better understanding about park-and-ride services that are common on transit networks in urban areas. In Transit Capacity and Quality of Service Manual (TRCP Report 164) Chapter 4, please review the material in pages 4-24 through 4-28, and write a 1-page summary to explain park-and-ride service, park and ride facilities, characteristics of park and ride users, and park and ride market areas.

Instead, if you want to review a technical report, journal article, or couple of news articles and then wanted to write a 1-page summary on any particular aspect relating to park-and-ride services; that should also be acceptable. In such situation, please provide the reference of materials you reviewed. This option may be a potential option for students who already have a good working knowledge about park-and-ride services. You don't need to do both. Just pick one option. 


\section{TL 787}

\section{Homework 6}

\section{Due: April 10 2019}

1. (15 points) A bus stop is located at a midblock on a two-way street having two through lanes in each direction. There is no parking on either sides of the roadway. The design of the bus stop is linear with three loading areas and the bus stop position is offline relative to the road and therefore the buses must exit the traffic stream to serve the passengers. The average number of boarding passengers is 10 passengers per bus and average number of alighting passengers is 12 passengers per bus. All the buses that stop in the bus stop are 40-foot low floor buses (that will facilitate level boarding at bus stop) that have a narrow front door (one channel) and a narrow rear door (one channel). Standees are not normally present on the bus. All boarding occurs through the front door and $60 \%$ of the passengers use monthly passes which needs visual inspection and the remaining $40 \%$ of passengers who purchase single ride or daily fares should use farebox with exact change. For percentage of passengers alighting through front door and rear door, use the recommendation provided from the bus capacity methodology in chapter 6 . Assume that the passengers waiting at the loading area is in crowded condition. Assume coefficient of variation of dwell times $\left(c_{v}\right)$ as 0.6 . Assume the bus door opening and close time as 4 seconds.

Assume a re-entry delay of 3 seconds for each bus to pull back into the traffic stream after serving the passengers. Using the design bus stop failure rate of $10 \%$, calculate the dwell time and loading area capacity for the bus stop. Follow and illustrate all the steps from steps 1-5 while solving dwell time and loading area capacity. When any needed data is not given, use the recommended values from Week 7 lecture notes or in the bus capacity methodology section of Chapter 6 in TCQSM $3^{\text {rd }}$ edition (pages 6-60 to 6-85).

2. (5 points) For the above situation, calculate the bus stop capacity. Assume that all the buses at the bus stop arrive at a random pattern. Also the bus stop is located far enough from a traffic signal that there is no influence for the bus stop from the queue of the stopped vehicles generated by the traffic signal. 


\section{TL 787}

\section{Homework 7}

Due: April 6th

1. (10 points) This is a reading assignment. The objective of this task is to get knowledge about some of the topics/concepts relating to rail transit capacity that were not covered in the lecture. Study Chapter 8 of Transit Capacity and Quality of Service Manual (TRCP Report 164) and write a brief summary (at least 4 sentences) for each of the following concepts.

a. Traffic Signal Priority for Transit Vehicles

b. Skip-Stop and Express Operation

c. Passenger-Actuated Doors

d. Fare Payment

e. Wheelchair Accommodations

2. (10 points) For the calculation example that is solved in the Week 11 recorded lecture, calculate the controlling headway and train throughput when there is a slope of $1.5 \%$ downgrade into the station and a $1.5 \%$ upgrade out of the station. There is no need to consider the effect of line voltage and hence the assume the value of $I_{v}$ as 1 (meaning 100\%). Assume an average dwell time at the critical station as 35 seconds and operating margin as 40 seconds. 


\section{TL 787}

\section{Homework $8^{1}$}

Due: April 15th

1. (5 points) A transit network is being designed to serve a $1500-m$-wide corridor. There should be two lines parallel to the corridor axis, and it is possible to locate them at any location.

a. What would be the optimal locations of the two lines to minimize access distances perpendicular to the lines, measuring from the left-hand boundary of the corridor?

b. What will be the average and maximum access distances, assuming that the population distribution in the corridor is uniform?

2. (5 points) List and define the advantages and disadvantages of trunk lines with feeders as compared to trunk lines with branches.

3. (5 points) How many transfer possibilities are there at a joint station with the following numbers of transit lines:

a. Three lines intersecting each other

b. Eight terminating lines

c. Three intersecting and two terminating lines.

4. (5 points) Define and briefly describe the three major objectives that should be considered in planning transit networks. Which three major parties are affected by the three major objectives?

\footnotetext{
${ }^{1}$ Source of homework problems: Vuchic, Vukan R. (2005) Urban Transit Operations, Planning, and Economics. Hoboken, New Jersey: John Wiley \& Sons, Inc.
} 


\section{TL 787 Public Transportation II \\ Term Project: Write a Transit-Related Paper \\ Assigned: January 28, 2016 \\ Final Report Due: May 10, 2016}

\section{Instructions}

For the term project, you should write a research paper on a public transportation topic. This project could be a qualitative analysis of a transit issue, a quantitative analysis of a transit-related data source, or a synthesis of previous research regarding a specific transit-related topic. Potential topics can include any transit-related topic. Search previous literature for ideas before selecting a topic. A list of potential topics can be provided by the instructor. The types of projects that can be conducted are described below.

Qualitative analysis: This could include a case study analysis, the creation and application of a framework, or some other type of qualitative analysis. This type of paper should also include references to at least four other research studies related to the chosen topic.

Quantitative analysis: This includes an analysis of a transit-related data source. Data could be obtained from the National Transit Database (http://www.ntdprogram.gov/ntdprogram/), a local transit agency, or some other source, or it could be collected yourself. This type of paper should also include references to at least four other research studies related to the chosen topic.

Synthesis report: A synthesis report is a summary of previous research conducted regarding a specific topic. In a synthesis report, you should summarize methods and key findings, compare and contrast the studies, identify and analyze key themes, and draw some conclusions. This type of paper should include references to at least ten research studies, though you do not need to go into an in-depth analysis of each study. Include at least 5-7 studies that you analyze in greater detail, while the other studies could be referenced for minor points or supporting arguments.

\section{Literature Review}

Each type of research project will require some type of literature review. You can search for literature through the TRID database (http://trid.trb.org/) or Google Scholar (scholar.google.com ), though you are not limited to these options. You can also try the World Transit Research site at http://www.worldtransitresearch.info/. At least half of your articles should include journal articles or TCRP reports, as these are generally of higher quality. If you find an article online that you do not have access to, email one of the instructors, and we may be able to access the article for you. 


\section{Due Dates}

The following are important due dates for the term project:

- Project Proposal Due: February 16

- The project proposal should be about 1-2 pages and it should provide a description of the topic you plan to write about and how you plan to complete the project.

- Draft Report Due: April 18

- Rough draft or detailed outline of complete paper

- Presentations: April 25-29

○ 15 minute PowerPoint presentation

- Final Report Due: May 10

\section{Manuscript Format}

Basic formatting guidelines are as follows:

- Overall Length: The length of the paper - including the abstract and references - should be a minimum of 3,500 words.

- Cover Page: The paper title, the author's name, the class name, and the date submitted should be included on the title page.

- Abstract: Following the cover page should be an abstract. The abstract must be no longer than 250 words, it must be self-contained, and it must not require reference to the paper to be understood.

- Page Setup: Students can use the font of their choice, but font size for the body of the text should be 10-12 points. Page numbers should be included. Page margins should be 1 inch.

- Tables \& Figures: Tables and figures should be embedded in the body of the report.

- References: The reference list should contain only references that are cited in the text, listed alphabetically. Denote a reference at the appropriate place in the text by citing the author name and year of publication.

Other formatting details are up to the student.

\section{Presentation Format}

Presentations should be about 15 minutes in length. Presentations will be followed by a brief question and answer session that will be moderated by the instructor. Tips for presentations can be found at the following link: http://onlinepubs.trb.org/onlinepubs/am/2015/AVTips.pdf 


\section{Grading}

The paper will be evaluated on the following criteria:

- Depth of coverage and completeness

- Clarity and organization

- Correctness (logical, computational, technical, grammatical)

- Findings/conclusions and lessons learned

- Neatness and professionalism

The overall grade for the project will be determined as follows:

- Project Proposal 5\%

- Draft Paper 10\%

- Presentation $30 \%$

- Final Paper 55\% 


\section{EXAMS}




\section{TL 787}

\section{Mid-Term Exam}

\section{Due: March 11, 2016}

1. (10 points) Describe the four main types of operating environments and how they affect transit speed, capacity, and reliability.

2. ${ }^{1}$ Operating speed on an 8- $\mathrm{km}$-long line is $V_{o}=16 \mathrm{~km} / \mathrm{hr}$. The headway is 12 minutes, and terminal time at each end is 6 minutes. Bus preferential treatments are being considered to improve the operation, but they would require a certain level of investment. The transit operator will make the investment if it can reduce the number of buses by one due to these improvements.

a. (10 points) What is the current number of buses needed for this bus line?

b. (10 points) What is the minimum operating speed that would allow saving one bus, assuming the headway remains at 12 minutes?

3. (10 points) Summarize the factors influencing transit ridership.

4. (10 points) Describe how elasticities and mode choice models can be used to predict changes in ridership following changes in service characteristics or external factors.

5. (7 points) Availability and Comfort and Convenience are the two important aspects in the transit trip decision making process. What are the key performance measures that are available in each aspect to evaluate the quality of service for fixed-route service and demand-response service?

6. a. (2.5 points) What is service coverage area?

b. ( 2.5 points) What are the values that are generally used for service coverage area for local bus stop and for rapid transit station?

c. ( 2.5 points) Explain walk distance based service coverage area and how it is different from air distance based coverage area?

d. (2.5 points) Explain the various street patterns and how walk distance based service areas varies for each type of street pattern.

7. (8 points) What are various sources of bus delay associated with bus stops? Briefly describe each source of bus delay.

\footnotetext{
${ }^{1}$ Source of problem: Vuchic, Vukan R. (2005) Urban Transit Operations, Planning, and Economics. Hoboken, New Jersey: John Wiley \& Sons, Inc.
} 
8. (25 Points) A bus facility in a non-CBD area of a metropolitan city consists of a series of five bus stops on a straight stretch of the road and is served by five transit routes with a combination peak hour frequency of 35 buses per hour. The straight stretch of the roadway is a three-lane one-way street and all the bus stops are linear and on-line design and located the near side of an intersection with $\mathrm{g} / \mathrm{C}$ ratio of 0.5 for all traffic signals. Determine the bus stop capacity for all the bus stops and bus facility capacity for a design failure rate of $7.5 \%$. Illustrate all the steps in the capacity calculation process.

Input Data for 5 bus stops on the bus facility:

\begin{tabular}{lrrrrr}
\hline & \multicolumn{5}{c}{ Bus Stops on Bus Facility } \\
& 1 & 2 & 3 & 4 & 5 \\
\hline Number of loading areas & 2 & 2 & 1 & 1 & 2 \\
Curb-lane volume $(\mathrm{veh} / \mathrm{h})$ & 350 & 450 & 300 & 400 & 500 \\
Right-turn volume $(\mathrm{v} / \mathrm{h})$ & 60 & 70 & 45 & 65 & 60 \\
Pedestrian volume $(\mathrm{v} / \mathrm{h})$ & 35 & 45 & 50 & 40 & 55 \\
Capacity of through movement $\left(\mathrm{C}_{\mathrm{th}}\right)(\mathrm{v} / \mathrm{h})$ & 900 & 900 & 900 & 900 & 900 \\
Reentry Delay (seconds) & 5 & 6 & 3 & 2 & 8 \\
Boarding Passengers & 8 & 10 & 4 & 5 & 9 \\
Alighting Passengers & 7 & 6 & 3 & 4 & 10 \\
\hline
\end{tabular}

All the buses that stop in the bus stop are 40-foot low-floor buses (that will facilitate level boarding at bus stop) that have a wide front door (two channel) and a narrow rear door (one channel). Standees are not normally present on the bus. All boarding occurs through the front door and $55 \%$ of the passengers use monthly passes which need visual inspection, and the remaining $45 \%$ of passengers who purchase single-ride or daily fares should use the farebox with exact change. For percentage of passengers alighting through front door and rear door, use the recommendations provided from the bus capacity methodology in chapter 6 of TCQSM.

Assume coefficient of variation of dwell times $\left(c_{v}\right)$ as 0.6 .

Assume the bus door opening and close time as 4 seconds.

Assume a boarding lost time as 3 seconds for bus stops with two loading areas. Also assume buses arrive in platoons at all bus stops.

When any needed data is not given, use the recommended values from Week 7 lecture notes or in the bus capacity methodology section of Chapter 6 in TCQSM 3rd edition (pages 6-60 to 6-85). 


\section{TL 787}

\section{Final Exam}

\section{Due: May 13, 2016}

1. (15 points) Discuss demand-response transit capacity issues and the factors you need to examine to determine needed capacity (e.g., number of vehicles and vehicle-hours needed).

2. (15 points) Discuss the differences between ridership and coverage goals, including the reasons for each goal, the tradeoffs, implications for service deployment, and performance measurement.

3. (12 points) Describe the types of errors that can arise when conducting transit surveys and how to minimize each.

4. (13 points) List and describe major factors of transit network design that mostly affect passenger attraction.

5. ${ }^{1}$ An urban sector has a transit network consisting of a trunk line and a number of branches, as shown below. This network is served by buses that come from the branches and continue on the trunk to the CBD.

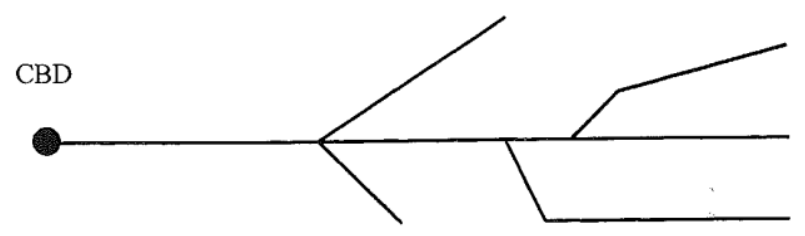

Discuss the advantages and disadvantages of changing the present operation into each one of the following alternatives:

a. (3 points) The same buses, but branches made to terminate at transfer points and the trunk line operate independently.

b. (3 points) The same as (a), but it is possible to obtain different types of buses for different lines; for example, articulated buses for the trunk, regular buses and minibuses for different feeders.

c. (3 points) The same as (a), but introduce a rail system (light rail transit [LRT] or rail rapid transit [RRT]) on the trunk.

(3 points) Which major factors would influence among the selection among the three alternatives?

\footnotetext{
${ }^{1}$ Source of problem: Vuchic, Vukan R. (2005) Urban Transit Operations, Planning, and Economics. Hoboken, New Jersey: John Wiley \& Sons, Inc.
} 
6. (13 points) List and describe the various rail track types. Discuss the construction materials/elements used for each track type, and locations where each track type is commonly used.

7. (20 points) Determine the control system's minimum train separation for both cab signaling system and moving block signaling system for the below situation.

For a heavy rail transit line, trains are planned to operate at a maximum speed of $65 \mathrm{mi} / \mathrm{h}$ and while entering the stations, a speed of $35 \mathrm{mi} / \mathrm{h}$ is maintained. Service is provided by ten-car trains with each car being $75 \mathrm{ft}$ long. Consider a slope of $1.5 \%$ downgrade into the station and a $1.5 \%$ upgrade out of the station. There is no need to consider the effect of line voltage and hence the assume the value of Iv as 1 (meaning 100\%). For all other variables, use the default values suggested from the TCQSM $3^{\text {rd }}$ Edition Chapter 8. 


\section{COURSE EVALUATIONS}




\section{TL 787: Public Transportation II}

\section{Evaluation of Spring 2016 Semester}

TL 787 Public Transportation II was offered for the first time in the Spring 2016 semester. The course was conducted strictly online. Originally 13 students registered, though one dropped within a couple weeks, another dropped shortly thereafter, and a third dropped in the middle of the semester. Among the ten remaining students, seven were actively involved in the class. The three other students took an incomplete for the course. Six of the actively involved students received an A for the course, and the other received a $\mathrm{B}$.

The class schedule closely followed the schedule outlined in the syllabus. The actual schedule of topics and the reference material from which the lectures were derived are shown in the Table below.

\begin{tabular}{|c|c|c|c|}
\hline Week & Topic & References & Instructor \\
\hline Jan 11-15 & Introduction, Mode and Service Concepts & TCQSM Chapters 2 & Jeremy \\
\hline Jan $18-22$ & Operations Concepts & TCQSM Chapter 3 & Jeremy \\
\hline Jan $25-29$ & Transit Operations and Service Scheduling & Vuchic Chapter 1 & Jeremy \\
\hline Feb 1-5 & Demand Analysis & $\begin{array}{l}\text { Balcombe Chapters } \\
3 \text { \& 5, Ceder } \\
\text { Chapter 11; TCRP } \\
161\end{array}$ & Jeremy \\
\hline Feb 8-12 & Quality of Service: Concepts and Methods & $\begin{array}{l}\text { TCQSM Chapters } \\
4,5\end{array}$ & Ranjit \\
\hline Feb 15-19 & Bus Transit Capacity & TCQSM Chapter 6 & Ranjit \\
\hline Feb 22-26 & Bus Transit Capacity & TCQSM Chapter 6 & Ranjit \\
\hline Feb 29-Mar 4 & Demand Response Transit Capacity & TCQSM Chapter 7 & Jeremy \\
\hline Mar 7-9 & Mid-term exam (No lecture) & & \\
\hline Mar 14-18 & No Class - NDSU Spring Break & & \\
\hline Mar 21-25 & Rail Transit Capacity & TCQSM Chapter 8 & Ranjit \\
\hline Mar 28-Apr 1 & Railroad Engineering & AREMA Material & Ranjit \\
\hline Apr 4-8 & Service Planning & Vuchic Chapter 4 & Ranjit \\
\hline Apr 11-15 & Transit Service Planning Issues & $\begin{array}{l}\text { Walker - Human } \\
\text { Transit }\end{array}$ & Jeremy \\
\hline Apr 18-22 & Transit Financing, Transit Surveys & $\begin{array}{l}\text { Innovative Funding } \\
\text { Sources for Transit, } \\
\text { Kockelman et al., } \\
\text { TCRP } 63\end{array}$ & Jeremy \\
\hline Apr 25-29 & Presentations & & \\
\hline May 2-6 & No class & & \\
\hline May 9-13 & Final exam, class project due & & \\
\hline
\end{tabular}

In most weeks, recorded lectures were provided in Blackboard using Tegrity. The length of the recorded lectures each week typically ranged from 1 hour to 1 hour 45 minutes (though they were sometimes 
broken into more than one part). Two live lectures were conducted. The first was on Railroad Engineering during the week of March 28 - April 1, and the second was on transit service planning issues during the week of April 11-15. The first was conducted on Blackboard using Collaborate and suffered from poor audio. The second was conducted with Adobe Connect and had much better audio, as well as better class attendance. The first live lecture only had three students attend (and only two for the entire lecture), while the second had five students attend.

Students were given eight homework assignments throughout the course of the class, and they typically had 1-2 weeks to complete each assignment. They were given a mid-term exam and final exam, both being take home exams, and a class project that required a final paper and class presentation. The students recorded their presentations using Tegrity. Class participation, which included use of the discussion board, counted for $10 \%$ of the final grade.

\section{Student Feedback}

At the end of the semester, students were asked to complete a standard course evaluation. In addition to this evaluation, we provided an additional survey to gather further student feedback regarding the content of the material covered in the class. Six students completed the course evaluation, and three completed the course content survey. Responses to the course evaluation are shown below.

Please rate your satisfaction with the instruction in this course.

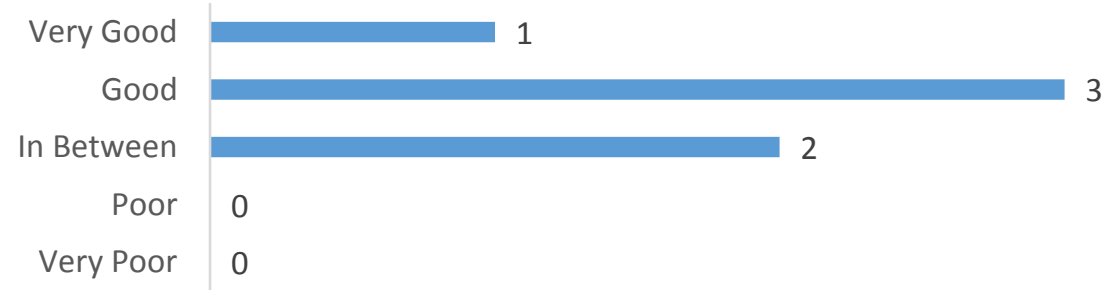

Please rate the instructor as a teacher.

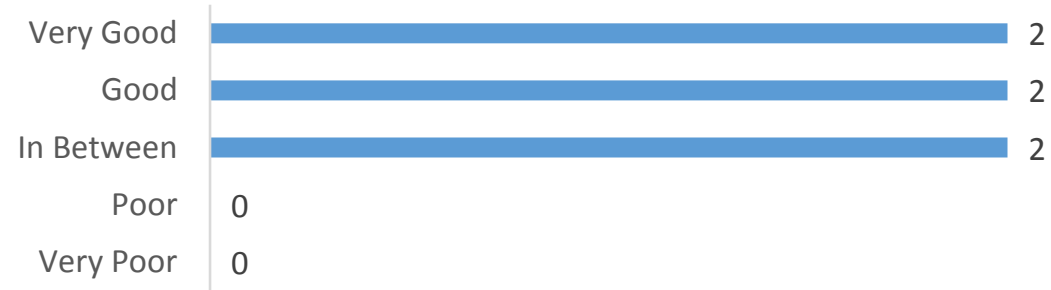

Please rate the ability of the instructor to communicate effectively.

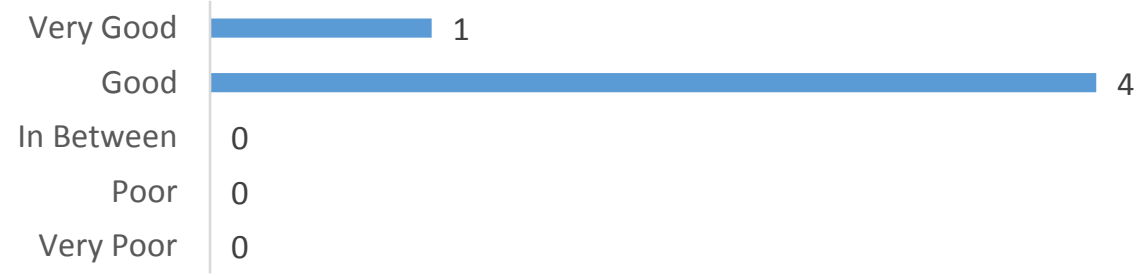


Please rate the quality of this course.

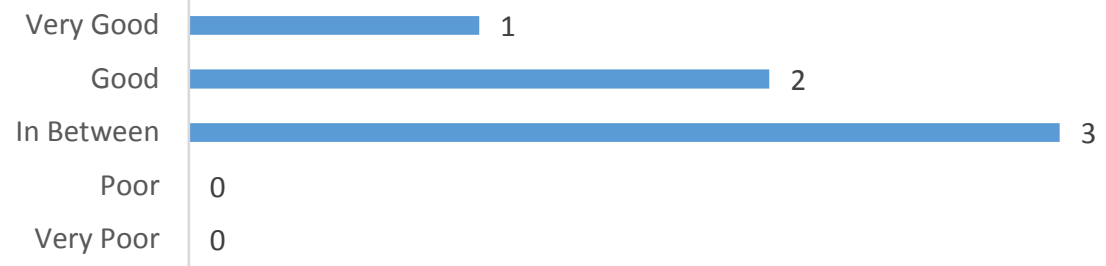

Please rate the fairness of procedures for grading this course.

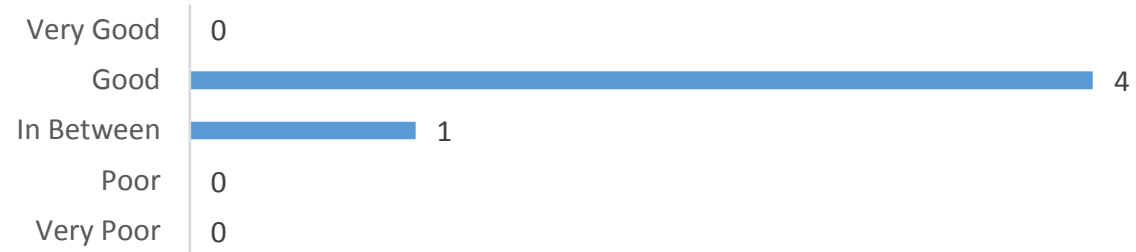

Please rate your understanding of the course content.

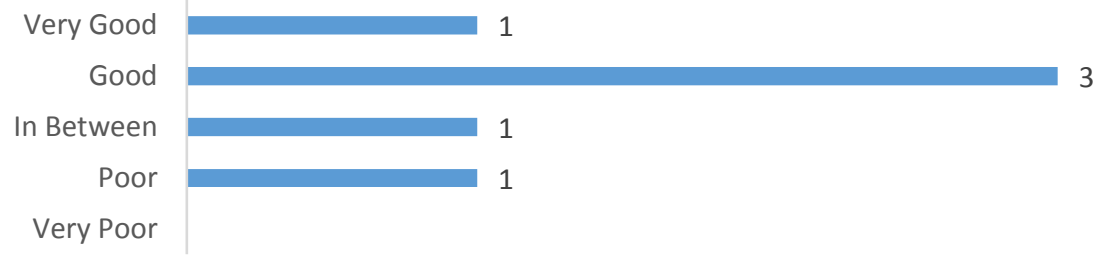

The course objectives were clearly stated.

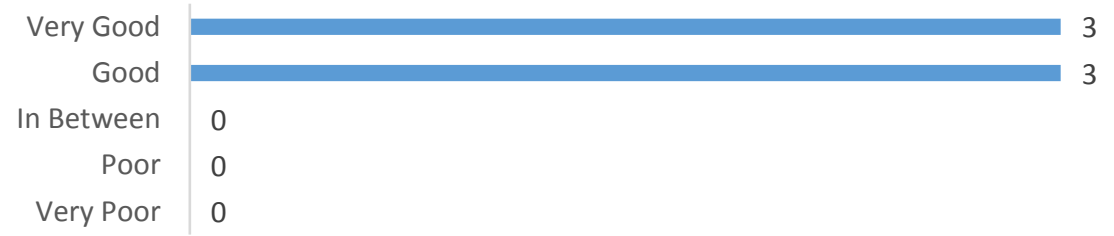

The coursework covered was pertinent to the course objectives.

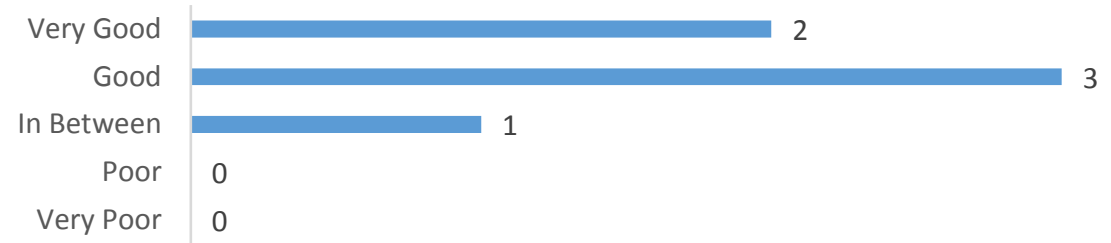


The examinations reflected class materials.

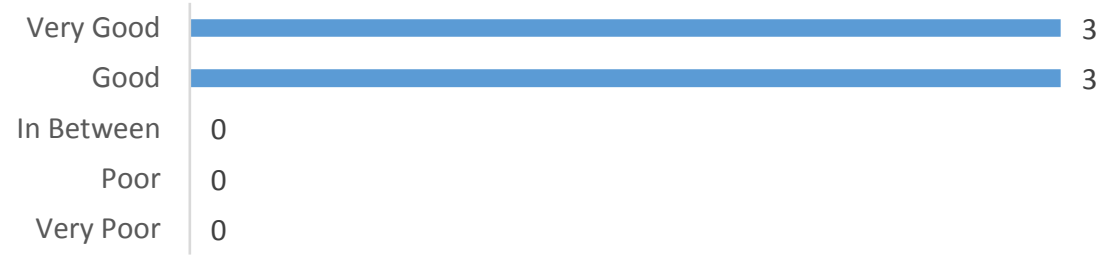

The instructor demonstrated a command of the subject matter.

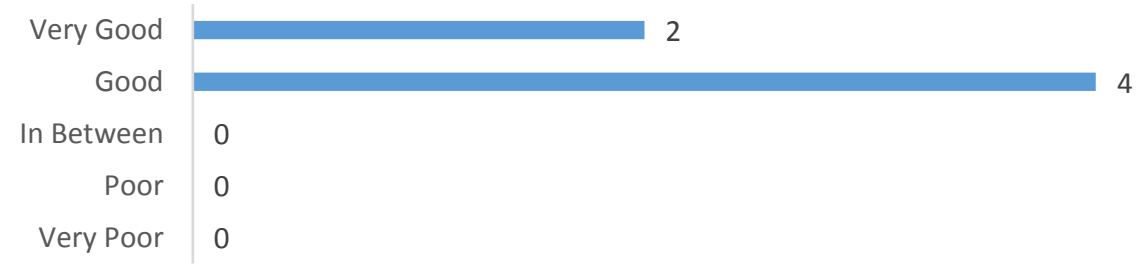

The instructor presented material at an appropriate intellectual level and was enthusiastic about the subject matter.

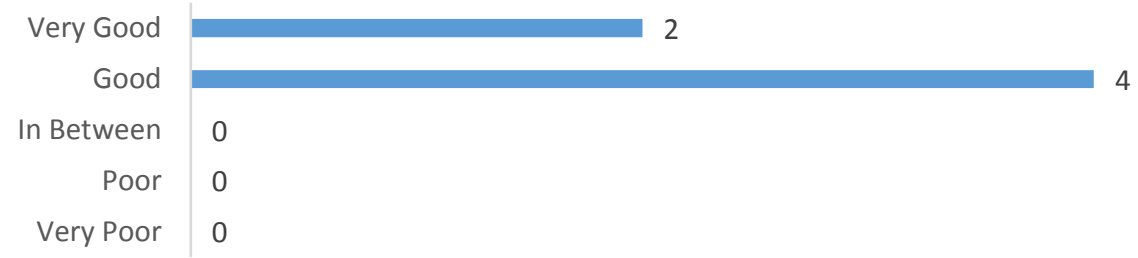

The instructor encouraged students' participation.

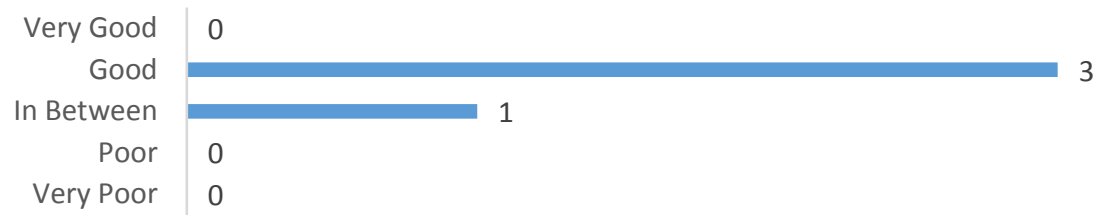

The instructor answered questions clearly.

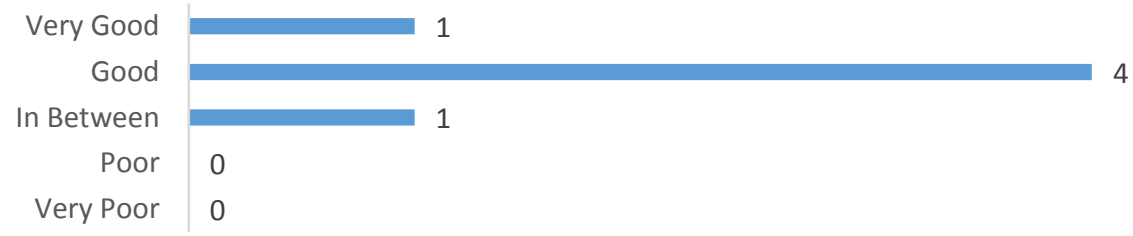


The instructor was available for students.

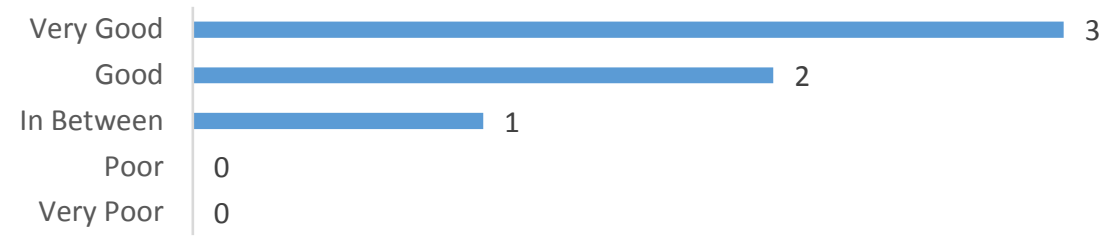

Please list at least ONE outstanding characteristic of this course.

- I liked that the instructors attempted to have live lectures.

- Provided the recorded lectures for future references and reviews for the homework assignments, papers, exams, and such. Provided assistant in a timely manner.

- This course contained significant analytical content that is not present in other courses.

- Tegrity presentations were easily digested.

- The course materials were good and relevant to what i want to know about transportation planning

Please list at least ONE aspect about this course that needs improvement and suggest a strategy for improvement.

- Sometimes the technology (Tegrity) seemed to not be working correctly.

- High frequency of the homework assignments that require decent amount of time. Recommend increasing the time of the due dates for the assignments.

- There should be a more content that relates to the theme of the course beyond purely mathematical approaches to public transportation. The course title may need to be changed to reflect an emphasis on engineering analysis and modeling.

- This course has heavy assignments in total, we have final projects, presentation, final exams and homeworks. Some of the homework questions are so difficult that takes too much time.

Personally, I think the instructor should balance the homework, projects, and exams with class participations.

- I wish the discussions had the ability to send daily digests or notifications.

- good to me so far

Was this course beneficial to you and your future career moves within the military, Department of Defense, or in the civilian sector.

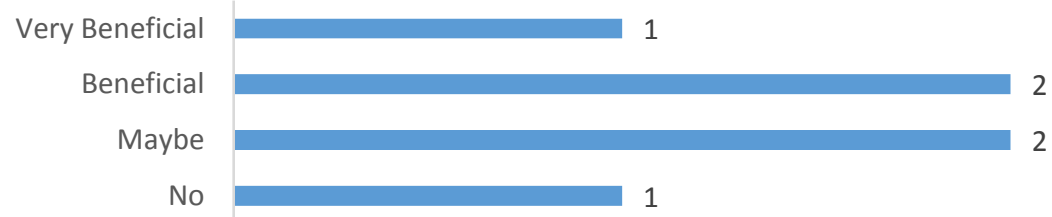


Was the content of this course relevant to your future positions and the future of military logistics around the world.

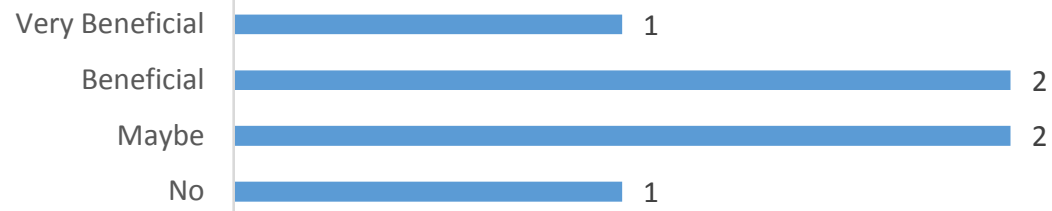

Responses to the course content survey are shown below. Only four students completed this survey, and one of the student's responses were not useful.

Which topic(s) covered in this class did you find most beneficial or interesting?

- none

- all the topics

- I found the topics on transit passenger volume analysis calculations and transit planning and investments to be the most beneficial to me.

- Bus Capacity and Demand Analysis

Were there any topics covered in this course that you would have liked to have seen covered in greater detail?

- no

- no

- I think more detail on transit planning topics would have been relevant to this course.

- Transit Operations and Service Scheduling

Were there any topics not covered in this course that you would have liked to have seen covered?

- no

- aviation planning

- I would like to see the more information on transit finances as they relate to real world practices that would have been helpful.

- Use of Advanced Technologies

Which topic(s) covered in this course did you find the least interesting or beneficial?

- no

- none

- It was the least beneficial for me going over the data collection methods, that might be better served in another class.

- Transit Financing

Provide any other comments about the content or structure of the course:

- no

- air transport planning should be introduced

- I think that some participation is helpful in encouraging communication between students.

- Overall, the course was conducted successfully. 


\section{Recommendations}

Based on the experience of the instructors and student feedback, we developed a number of recommendations for improving the course.

\section{Increase student interaction and use of the discussion board}

The discussion board can be used to a greater extent to encourage interaction between students, as well as between students and the instructors. The discussion board was used in the class but was not used very extensively until near the end of the semester. After giving class presentations, students were required to provide comments and questions to other students about their presentation on the discussion board. This proved to be very successful in encouraging interaction between the students. Similar exercises like this should be conducted throughout the semester. Homework assignments could be given that require use of the discussion board.

\section{Conduct more live classes}

The recorded lectures work well, but providing live classes allow students the opportunity to interact with the instructor or other students. When polled, most students answered that they were somewhat interested in having live classes, indicating that it may not be most important but they see some benefit to it. Finding a time that most students could attend the class was difficult, as they all had full-time jobs and other commitments, but students who could not attend were able to view a recording of the lecture.

\section{Rearrange the order of topics covered}

The lecture near the end of the semester on transit service planning issues was a less technical lecture that would have worked well as more of an introductory lecture earlier in the semester. We should consider if we should do any further rearranging, such as moving service planning from Vuchic up earlier in the semester, though I think the order worked generally well.

\section{Cover service planning more, and possibly demand analysis}

We were not able to cover all of chapter 4 from Vuchic on service planning, though I think this is an important topic. We could spend an additional week on this topic and cover the entire chapter. We could also consider adding another week on demand analysis. One student mentioned that the transit planning and demand analysis topics were the most beneficial, and the class could benefit from providing greater coverage of these topics.

\section{Consider adding more material on transit finances and investments}

One student recommended additional coverage of transit finances and investments. One brief lecture was given on this topic. This is an important topic, and the class would benefit from covering it in greater detail. Unfortunately, this is a difficult subject to cover and is not an area of expertise for either instructor. However, we can study this issue more and investigate how it could be covered better in the class.

\section{Cut lecture on transit surveys and data collection}

While conducting transit surveys and collecting data is important, this topic may not fit as well in this class and could be cut if we plan to extend coverage of other topics. The lecture on railroad engineering could also be cut if needed because some of this material is covered in another course. 


\section{Consider renaming course to provide greater detail}

One student commented that "the course title may need to be changed to reflect an emphasis on engineering analysis and modeling." This course is much more analytical and quantitative than the preceding course, TL 786 Public Transportation I. Although the more quantitative nature of the course is noted in the course description, some students may have expected the class to be more like TL 786 and were not expecting the more analytical and quantitative material. The course title could be changed to something more descriptive to ensure more accurate expectations. Alternatively, the course description could be edited to more clearly describe the analytical and quantitative nature. A possible alternative course name would be something like Public Transportation Engineering and Analysis or Public Transportation: Planning, Analysis, and Design. The course title, though, needs to be inclusive of all topics covered, and if we add more material on finances, it may not fit under a new course title such as these.

\section{Evaluate assignments}

The workload for the course seems appropriate, compared to other courses in the program, though a couple students did comment on the heavy workload. The workload is heavier than that for some other courses in the program, but other courses in the program have a similar or even greater workload. The workload consisted of eight homework assignments, two take-home exams, and a class project that required a final paper and class presentation. This may be fine, but we could consider alternative approaches, such as fewer homework assignments with more time to work on them but with more questions. The class projects worked well and allowed students to explore different topics and share their work with other students. However, there was somewhat of a disconnect between the class project and the material covered in the class. That's not necessarily bad, as it broadened the range of topics studied, and the projects were mostly well done, but the class project seemed like it did not fit quite as well with the theme for the rest of the class. Options are to adjust the class project so that it focuses more on the topics covered in class, drop the class project and increase the workload for other assignments, or keep it as is. 